INSTITUT FÜR PFLANZENBAU UND TIERPRODUKTION

IN DEN TROPEN UND SUBTROPEN

GEORG -AUGUST UNIVERSITÄT GÖTTINGEN

\title{
EINFLUSS DER SCHWERMETALLE ZINK, CADMIUM UND BLEI AUF WACHSTUM UND NÄHRSTOFFAUFNAHME VON Acacia saligna, Casuarina equisetifolia UND Cupressus sempervirens
}

\author{
Dissertation \\ Zur Erlangung des Doktorgrades \\ der Fakultät für Agrarwissenschaften \\ der Georg-August-Universität Göttingen
}

vorgelegt von

Ragaa Aly Taha Aly

geboren in El -Minia Ägypten

Göttingen, Mai 2002 
D 7

1. Referentin / Referent : Prof. Dr. P.L.G. Vlek

2. Korreferentin/Korreferent : Prof. Dr. W. Römer

Tag der mündlichen Prüfung : 16 Mai 2002 


\section{Im Text häufig verwendete Abkürzungen :}

Abb.

CAL

Demi -Wasser

DL

DSM - Medium

$-\mathrm{F}$

$+\mathrm{F}$

GD

Kap.

Konz.

$\min$.

MW

N-Z-Amine A

ns

$-\mathrm{R}$

$+\mathrm{R}$

Rhiz.

STG

Tab.

TS

WTG
Abbildung

Calcium-acetat-Lactat

demineralisiertes Wasser

Doppellactat

Medium aus Catalogue of Strains DSM (Deutsche Sammlung von Mikroorganismen und Zellkulturen $\mathrm{GmbH}$ )

ohne Frankia

mit Frankia

Grenzdifferenz

Kapitel

Konzentration

Minute

Mittelwert

Casein enzymatic hydrolysate

nicht signifikant

ohne Rhizobien

mit Rhizobien

Rhizobien

Sproßtrockengewicht

Tabelle

Trockensubstanz

Wurzeltrockengewicht 


\section{Inhaltsverzeichnis}

Seite

1 Einführung 1

1. 1 Einleitung 4

2 Konkrete Ziele der vorliegenden Arbeit und Lösung 5

3 Literaturüberblick 5

3.1 Anmerkungen zu wichtigen holzliefernden Bäumen in Ägypten 11

4 Material und Methodik 14

4.1 Boden und Bodenvorbereitung 14

4.2 Versuchsräume und Gefäße 14

4.3 Versuchspflanzen und Pflanzenanzucht 16

$\begin{array}{ll}4.4 \text { Düngung } & 17\end{array}$

4.5 Bewässerung 18

4.6 Schwermetallgaben 18

4.7 Vermehrung und Beimpfung mit Rhizobien und Frankia 19

$\begin{array}{ll}4.8 \text { Versuchauswertung } & 21\end{array}$

$\begin{array}{lll}4.8 .1 & \text { Ernte } & 21\end{array}$

$\begin{array}{ll}\text { 4.8.2 Mineralstoffanalyse } & 21\end{array}$

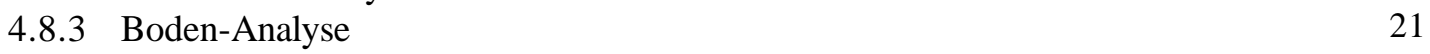

$\begin{array}{lll}\text { 4.8.4 Statistische Auswertung } & 21\end{array}$

5 Ergebnisse $\quad 22$

5.1 Versuch $1 \quad 22$

5.1.1 Acacia saligna 22

$\begin{array}{ll}\text { 5.1.1.1 Zn-Behandlung } & 22\end{array}$

$\begin{array}{lll}\text { 5.1.1.1.1 Sprosstrockengewicht } & 22\end{array}$

5.1.1.1.2 Wurzeltrockengewicht 22

5.1.1.1.3 Konzentration und Aufnahme von N, P, K, Ca, Mg und Zn im Sproß 23

5.1.1.1.4 Zn-Konzentration und Aufnahme in die Wurzel 26

5.1.1.1.5 Der pH-Wert des Boden 27

5.1.1.2 Cd-Behandlung 28

$\begin{array}{lll}\text { 5.1.1.2.1 Sprosstrockengewicht } & 28\end{array}$

5.1.1.2.2 Wurzeltrockengewicht 28

5.1.1.2.3 Konzentration und Aufnahme von N, P, K, Ca, Mg und Cd im Sproß 29

5.1.1.2.4 Cd-Konzentration und Aufnahme in der Wurzel 31

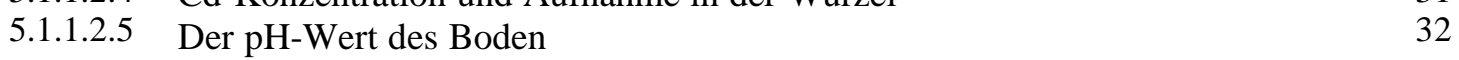

$\begin{array}{ll}\text { 5.1.1.3 Pb-Behandlung } & 33\end{array}$

\begin{tabular}{lll} 
5.1.1.3.1 Sprosstrockengewicht & 33 \\
\hline & 5.1.1. & 33
\end{tabular}

5.1.1.3.2 Wurzeltrockengewicht 33

5.1.1.3.3 Konzentration und Aufnahme von N, P, K, Ca, Mg und Pb im Sproß 33

5.1.1.3.4 Pb-Konzentration und Aufnahme in der Wurzel 36

5.1.1.3.5 Der pH-Wert des Boden 37

5.1.2 Casuarina equisetifolia 38

5.1.2.1 Zn-Behandlung 38

$\begin{array}{lll}\text { 5.1.2.1.1 Sprosstrockengewicht } & 38\end{array}$

5.1.2.1.2 Wurzeltrockengewicht 38 
5.1.2.1.3 Konzentration und Aufnahme von N, P, K, Ca, Mg und Zn im Sproß 39

5.1.2.1.4 Zn-Konzentration und Aufnahme in der Wurzel 41

5.1.2.1.5 Der pH-Wert des Boden $\quad 42$

5.1.2.2 Cd-Behandlung $\quad 43$

$\begin{array}{lll}\text { 5.1.2.2.1 Sprosstrockengewicht } & 43\end{array}$

5.1.2.2.2 Wurzeltrockengewicht 43

5.1.2.2.3 Konzentration und Aufnahme von N, P, K, Ca, Mg und Cd im Sproß 43

5.1.2.2.4 Cd-Konzentration und Aufnahme in der Wurzel 46

$\begin{array}{ll}\text { 5.1.2.2.5 Der } \mathrm{pH}-\text { Wert des Boden } & 47\end{array}$

$\begin{array}{ll}\text { 5.1.2.3 Pb-Behandlung } & 48\end{array}$

$\begin{array}{lll}\text { 5.1.2.3.1 Sprosstrockengewicht } & 48\end{array}$

5.1.2.3.2 Wurzeltrockengewicht 48

5.1.2.3.3 Konzentration und Aufnahme von N, P, K, Ca, Mg und Pb im Sproß 49

5.1.2.3.4 Pb-Konzentration und Aufnahme in der Wurzel 51

5.1.2.3.5 Der pH-Wert des Boden $\quad 52$

5.1.3 Cupressus sempervirens

5.1.3.1 Zn-Behandlung 53

$\begin{array}{lll}\text { 5.1.3.1.1 } & \text { Sprosstrockengewicht } & 53\end{array}$

5.1.3.1.2 Wurzeltrockengewicht 53

5.1.3.1.3 Konzentration und Aufnahme von N, P, K, Ca, Mg und Zn im Sproß 54

5.1.3.1.4 Zn-Konzentration und Aufnahme in der Wurzel 56

$\begin{array}{ll}\text { 5.1.3.1.5 Der } \mathrm{pH}-\text { Wert des Boden } & 57\end{array}$

$\begin{array}{ll}\text { 5.1.3.2 Cd-Behandlung } & 58\end{array}$

$\begin{array}{lll}\text { 5.1.3.2.1 } & \text { Sprosstrockengewicht } & 58\end{array}$

$\begin{array}{lll}\text { 5.1.3.2.2 Wurzeltrockengewicht } & 58\end{array}$

5.1.3.2.3 Konzentration und Aufnahme von N, P, K, Ca, Mg und Cd im Sproß $\quad 58$

5.1.3.2.4 Cd-Konzentration und Aufnahme in der Wurzel 61

$\begin{array}{lll}\text { 5.1.3.2.5 Der } \mathrm{pH}-\text { Wert des Boden } & 62\end{array}$

5.1.3.3 Pb-Behandlung 63

$\begin{array}{lll}\text { 5.1.3.3.1 } & \text { Sprosstrockengewicht } & 63\end{array}$

$\begin{array}{lll}\text { 5.1.3.3.2 Wurzeltrockengewicht } & 63\end{array}$

5.1.3.3.3 Konzentration und Aufnahme von N, P, K, Ca, Mg und Pb im Sproß 64

5.1.3.3.4 Pb-Konzentration und Aufnahme in der Wurzel 66

$\begin{array}{lll}\text { 5.1.3.3.5 Der pH-Wert des Boden } & 67\end{array}$

\subsection{Versuch 2}

Zusammenfassender Überblick über die Ergebnisse von Versuch 1

5.2.1 Acacia saligna 72

$\begin{array}{lll}\text { 5.2.1.1 Sprosstrockengewicht } & 72\end{array}$

$\begin{array}{lll}\text { 5.2.1.2 Wurzeltrockengewicht } & 72\end{array}$

5.2.1.3 Konzentration und Aufnahme von N, P, K, Ca, Mg und Pb im Sproß 73

5.2.1.4 Pb-Konzentration und Aufnahme in die Wurzel 76

5.2.1.5 Der pH-Wert des Boden $\quad 76$

5.2.2 Casuarina equisetifolia

$\begin{array}{lll}\text { 5.2.2.1 Sprosstrockengewicht } & 77\end{array}$

$\begin{array}{lll}\text { 5.2.2.2 Wurzeltrockengewicht } & 77\end{array}$

5.2.2.3 Konzentration und Aufnahme von N, P, K, Ca, Mg und Pb im Sproß 77

5.2.2.4 Pb-Konzentration und Aufnahme in der Wurzel 80

5.2.2.5 Der pH-Wert des Boden $\quad 81$

5.2.3 Cupressus sempervirens 82

$\begin{array}{lll}\text { 5.2.3.1 Sprosstrockengewicht } & 82\end{array}$

$\begin{array}{lll}\text { 5.2.3.2 Wurzeltrockengewicht } & 82\end{array}$

5.2.3.3 Konzentration und Aufnahme von N, P, K, Ca, Mg und Pb im Sproß 82

5.2.3.4 Pb-Konzentration und Aufnahme in der Wurzel 85

5.2.3.5 Der pH-Wert des Boden 86

Zusammenfassender Überblick über die Ergebnisse von Versuch 2 
5.3.1.1 Zn-Behandlung 91

$\begin{array}{lll}\text { 5.3.1.1.1 Sprosstrockengewicht } & 91\end{array}$

5.3.1.1.2 Wurzeltrockengewicht 92

5.3.1.1.3 Konzentration und Aufnahme von N, P, K, Ca, Mg und Zn im Sproß 92

5.3.1.1.4 Zn-Konzentration und Aufnahme in der Wurzel 96

$\begin{array}{ll}\text { 5.3.1.1.5 Der pH-Wert des Boden } & 97\end{array}$

$\begin{array}{ll}\text { 5.3.1.2 Cd-Behandlung } & 98\end{array}$

$\begin{array}{lll}\text { 5.3.1.2.1 Sprosstrockengewicht } & 98\end{array}$

$\begin{array}{lll}\text { 5.3.1.2.2 Wurzeltrockengewicht } & 98\end{array}$

5.3.1.2.3 Konzentration und Aufnahme von N, P, K, Ca, Mg und Cd im Sproß 99

5.3.1.2.4 Cd-Konzentration und Aufnahme in der Wurzel 102

$\begin{array}{ll}\text { 5.3.1.2.5 Der pH-Wert des Boden } & 103\end{array}$

$\begin{array}{ll}\text { 5.3.1.3 Pb-Behandlung } & 104\end{array}$

$\begin{array}{lll}\text { 5.3.1.3.1 } & \text { Sprosstrockengewicht } & 104\end{array}$

$\begin{array}{lll}\text { 5.3.1.3.2 } & \text { Wurzeltrockengewicht } & 104\end{array}$

5.3.1.3.3 Konzentration und Aufnahme von N, P, K, Ca, Mg und Pb im Sproß 105

5.3.1.3.4 Pb-Konzentration und Aufnahme in der Wurzel 108

$\begin{array}{ll}\text { 5.3.1.3.5 Der pH-Wert des Boden } & 109\end{array}$

$\begin{array}{ll}\text { 5.3.2 Casuarina equisetifolia } & 110\end{array}$

Zusammenfassender Überblick über die Ergebnisse von Versuch $3 \quad 120$

6 Diskussion $\quad 124$

$\begin{array}{lr}\text { 6.1 Schlussfolgerung } & 134\end{array}$

7 Zusammenfassung 136

8 Literatur 139 


\section{Einführung}

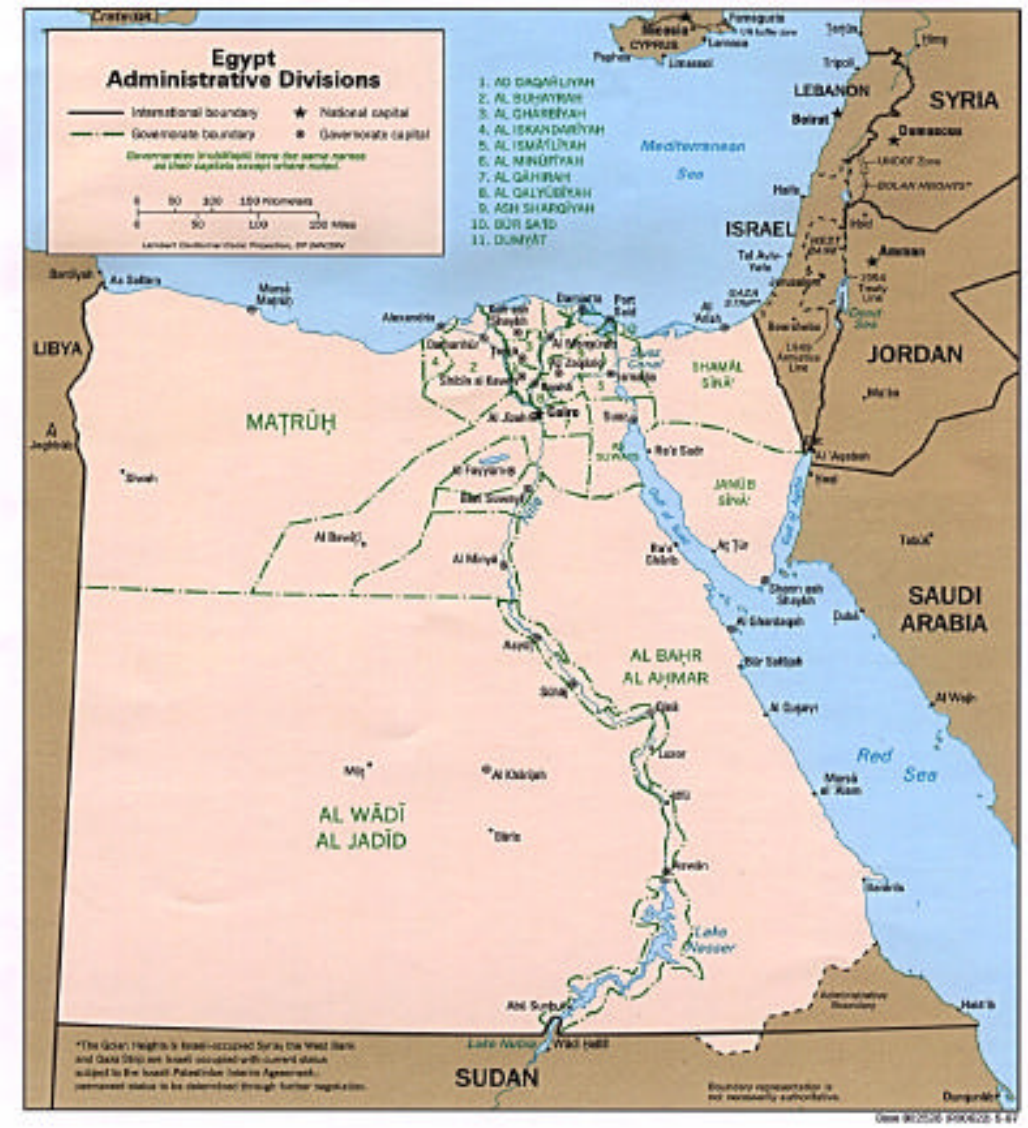

\section{Einige allgemeine Angaben zu Ägypten *}

Ägypten, amtlich Arabische Republik Ägypten (zwischen 1958 und 1971 Vereinigte Arabische Republik), liegt im Nordosten Afrikas Das Land wird im Norden begrenzt durch das Mittelmeer; im Osten grenzt es an Israel und das Rote Meer, im Süden an den Sudan und im Westen an Libyen. Die größten Abmessungen des Staates betragen in Nordsüdrichtung 1.085 Kilometer und im Süden des Staates in Ostwestrichtung 1.255 Kilometer.

* ( Die folgenden Angaben stammen aus:

Die Chronik der Menschheit, Bertelsmann-Verlag, Gütersloh, 1997 Brockhaus; Bibliographisches Institut \& F.A. Brockhaus AG, 1999

Microsoft ${ }^{\circledR}$ Encarta ${ }^{\circledR} 97$ Enzyklopädie. 1993-1996 Microsoft Corporation

Microsoft ${ }^{\circledR}$ Encarta ${ }^{\circledR}$ Weltatlas 99. 1995 ) 
Die Gesamtfläche beträgt 997.738 Quadratkilometer. Die Hauptstadt ist Kairo. Das ägyptische Niltal gilt als die Wiege einer der am höchsten entwickelten Kulturen des Altertums, deren Geschichte sich über historische Quellen bis etwa 3.200 v. Chr. zurückverfolgen lässt.

\section{Klima und Vegetation}

\section{Landesnatur}

Die $1.550 \mathrm{~km}$ lange und 1 bis $20 \mathrm{~km}$ breite Stromoase des Nils, sein Mündungsdelta, die Senke von Faijum sowie die übrigen Oasen sind landwirtschaftlich nutzbar (das heißt bewässert) und besiedelt. Das gesamte Kultur- und Siedlungsland nimmt nur 3,5 Prozent der Staatsfläche ein. Westlich des Niltals erstreckt sich das Tafelland der Libyschen Wüste mit einer durchschnittlichen Höhe von $1.000 \mathrm{~m}$ über dem Meeresspiegel. Einzelne Oasen liegen in Senken zum Teil unter Meeresniveau, wie die Kattarasenke (bis $137 \mathrm{~m}$ unter dem Meeresspiegel). Im Osten breitet sich das Arabische Wüste aus, die in steiler, über $1.000 \mathrm{~m}$ hoher Stufe zum Roten Meer abfällt. Die ebenfalls wüstenhafte Halbinsel Sinai (bis 2.637 m über dem Meeresspiegel) gehört geographisch bereits zu Vorderasien.

\section{Klima}

Das Klima in Ägypten wird von einer heißen Jahreszeit zwischen Mai und September und einer kühlen Jahreszeit von November bis März bestimmt. Vorherrschende Nordwinde sorgen dafür, dass es in beiden Jahreszeiten kaum zu Extremtemperaturen kommt. In der Küstenregion liegen die Temperaturen zwischen einem durchschnittlichen Höchstwert von $37,2^{\circ} \mathrm{C}$ und einem durchschnittlichen Tiefstwert von $13,9^{\circ} \mathrm{C}$. In den Wüstengebieten kann es zu extremen Temperaturunterschieden zwischen Tag und Nacht kommen, von einem durchschnittlichen Jahreshöchstwert von $45,6^{\circ} \mathrm{C}$ während des Tages zu einem durchschnittlichen Jahrestiefstwert von $5,6^{\circ} \mathrm{C}$ nach Einbruch der Dunkelheit. Während des Winters fallen die Temperaturen in der Wüste oft auf Temperaturen um $0^{\circ} \mathrm{C}$. Die niederschlagsreichsten Gebiete befinden sich an der Mittelmeerküste, wo die jährliche Niederschlagsmenge bei $200 \mathrm{~mm}$ liegt. Weiter nach Süden hin nimmt die Niederschlagsmenge rasch ab, in Kairo beträgt sie nur mehr $28 \mathrm{~mm}$ pro Jahr, und in vielen Wüstengebieten kommt es nur alle paar Jahre zu Niederschlägen.

Die Sommer sind heiß und trocken, die Winter mild mit geringen Niederschlägen im Norden. Im Frühjahr treten heiße Sandstürme auf. 


\section{Landwirtschaft}

Ägypten ist ein vorwiegend agrarisch geprägtes Land; etwa 40 Prozent der erwerbstätigen Bevölkerung leben von Landwirtschaft und Viehzucht. Der Grundbesitz wurde durch das Landreformgesetz von 1952 grundlegend umstrukturiert und der individuelle Bodenbesitz auf eine Fläche von 80 Hektar begrenzt, 1961 auf 40 Hektar verringert und 1969 noch einmal auf 20 Hektar halbiert. Die vom Staat beschlagnahmten Ländereien wurden zwar an die Fellachen verteilt, doch das wirtschaftliche Gefälle zwischen mittelständischen Bauern und Fellachen besteht unverändert fort. Auf Betreiben der Regierung wurde die Gesamtfläche des landwirtschaftlich nutzbaren Landes durch Urbarmachung, Bewässerung, insbesondere seit Abschluss der Bauarbeiten am Assuan-Hochdamm 1970, dem Einsatz moderner Technologie, Kunstdünger und landwirtschaftlichen Geräten, vergrößert. Das ägyptische Kulturland gehört zu den ertragreichsten Böden der Welt. Ägypten ist der weltweit wichtigste Produzent von langfaseriger Baumwolle. Die Jahresproduktion an Baumwolle betrug $\mathrm{zu}$ Beginn der neunziger Jahre 294.000 t. Mit einer Jahresproduktion von 5,2 Millionen t gehört Ägypten auch $\mathrm{zu}$ den führenden Maisproduzenten. Weitere wichtige Produkte sind Zuckerrohr (11 Mio. t), Weizen (4,4 Mio. t), Reis (3,1 Mio. t) und Tomaten (1,5 Mio. t). Daneben werden Wassermelonen, Hirse, Gerste, Zwiebeln, Gemüse, Zitrusfrüchte, Mangos, Datteln, Feigen und Wein angebaut.

Der Nahrungsmittelbedarf der Bevölkerung wird allerdings nicht aus eigener Produktion gedeckt, so dass rund zwei Drittel der Nahrungsmittel (vor allem Weizen und Fleisch) eingeführt werden müssen.

\section{Außenhandel}

$\mathrm{Zu}$ den wichtigsten Importgütern gehören landwirtschaftliche Produkte und Lebensmittel, Transportfahrzeuge, chemische Stoffe, Bergbau- und Steinbruchmaschinen sowie Metallwaren. Die Haupthandelspartner sind nach den EU-Ländern die USA, Australien und Japan. Aufgrund des rapiden Bevölkerungswachstums wurde das Land immer mehr von Lebensmittelimporten abhängig, insbesondere von Weizen, Mehl und Fleisch. $\mathrm{Zu}$ den wichtigsten Exportgütern zählen Rohöl und Erdölprodukte, Rohbaumwolle, Baumwollgarn und -stoffe sowie Nahrungsmittel. Die Hauptabnehmerländer für diese Erzeugnisse sind Italien, Rumänien, Deutschland, Großbritannien, Frankreich und Japan. 


\subsection{Einleitung}

Der Landbau in den tropischen und subtropischen Gebieten steht vor besonders gravierenden Problemen, will man dort effizienten, ökologisch angepassten Pflanzenbau betreiben. Hauptnutzungsprobleme in diesen Zonen, wie auch in Ägypten, sind Wasserknappheit und hohe Temperaturen. Diese Faktoren verursachen einen enormen Schwund fruchtbaren Bodens vorrangig durch Winderosion (Sandstürme aus der Sahara). Die Steigerung der Nahrungsmittelproduktion trotz dieser und anderer ökologischer Benachteiligungen (Nährstoffmangel, Wassermangel u.a.) (Weischet, 1984), gehört daher immer noch zu den Hauptaufgaben der vornehmlich in den Tropen und Subtropen liegenden Entwicklungsländer. In Ägypten, dem am dichtesten besiedelten Land Afrikas, mit mehr als 66 Millionen Einwohnern werden $8 \%$ der gesamten Fläche für den Anbau von Kulturpflanzen genutzt. Das heißt, die Gesamtfläche des landwirtschaftlich nutzbaren Landes wurde in den letzten Jahrzehnten vergrößert, insbesondere seit Abschluss der Bauarbeiten am Assuan-Hochdamm (1970). Die Restfläche ist Wüste. In Ägypten ist jedoch eine Steigerung der landwirtschaftlichen Produktion durch verbesserte Integration von Ackerbau und Tierhaltung möglich. Die Nutzung und Verbesserung der Flächen durch den Anbau geeigneter Futterpflanzen ist ebenfalls ein wichtiger Gesichtspunkt in Ägypten. Obwohl der Anbau von Stärke-, Zucker-, Öl-, Eiweiß-, Faser-, Gemüse- und Obst-Pflanzen Vorrang vor den Holzbäumen hat, stellen diese eine sehr wichtige Alternative zu den genannten Kulturen dar, insbesondere in den trockenen Gebieten und /oder auf Böden, die mit Schwermetallen wie Zn, $\mathrm{Cd}$ und $\mathrm{Pb}$, besonders in Industriegebieten um die großen Städte, wie Kairo und Alexandria, verseucht sind. Aus Erfahrungen der Leute in Ägypten ist bekannt, dass die Eignung dieser Bäume in erster Linie auf deutlich geringeren Ansprïchen an Wasser und Nährstoffen beruht. Sie sind darüber hinaus die wichtigsten natürlichen Lieferanten für Holz.

Noch im vorigen Jahrhundert wuchs in Ägypten eine große Anzahl von Holzbäumen verschiedener Arten. Vor allem durch den Einfluss der ständig wachsenden Bevölkerung im Lande sank diese Anzahl drastisch ab (Badran und Kandeel, 1971). Heute stehen nur geringe Anbauflächen für Bäume zur Holzgewinnung zur Verfügung: Straßenränder, Bewässerungsgräben, Gartenzäune und ähnliches. Deshalb werden z.Z. in Ägypten verschiedene Maßnahmen zur Steigerung der Rohholzproduktion geprüft. Zu diesen Maßnahmen zählt der Anbau von Holzbäumen auf marginalen, nährstoffarmen und darüber hinaus auf schwermetallkontaminierten Böden in den Industriegebieten Ägyptens. Dabei ist weitgehend unbekannt, welche Baumarten gegenüber bestimmten Schwermetallen tolerant sind und gutes Wachstum zeigen. Es wäre also für die Planung und Durchführung von 
Baumpflanzungen gut zu wissen, welche Baumarten für diese Situationen geeignet sind. Aus dieser Situation leitet sich die Zielstellung für die vorliegende Arbeit ab. Sie soll untersuchen, welche von drei ausgewählten Baumarten gegenüber den drei Schwermetallen $\mathrm{Zn}, \mathrm{Cd}$ und $\mathrm{Pb}$ am tolerantesten ist.

\section{Konkrete Ziele der vorliegenden Arbeit und Lösungsweg:}

1. Ermittlung der Wirkung gestaffelter Gaben der Schwermetalle $\mathrm{Zn}, \mathrm{Cd}$ und $\mathrm{Pb}$ zum Boden auf Wachstum und Nährstoffaufnahme der in Ägypten meist angebauten Holzbäume Acacia saligna, Casuarina equisetifolia und Cupressus sempervirens, um die Baumarten, zu selektieren, die unter der Wirkung eines der Schwerme talle noch am besten wachsen kann.

2. Beantwortung der Frage, ob die Inokulation von Acacia- und Casuarina-Pflanzen mit $\mathrm{N}_{2}$-fixierenden Bakterien- mit Rhizobien bei A. saligna und Actinomycten (Frankia) bei C. equisetifolia, das Wachstum dieser Pflanzen verbessern kann, wenn sie in mit einem Schwermetall kontaminierten Boden wachsen.

Damit stellt diese Arbeit einen ersten Schnitt zur Erforschung des Problems der Nutzung von mit Schwermetallen kontaminierten Böden durch den Anbau von holzliefernden Bäumen, bzw. einen Beitrag zum Umweltschutz in Ägypten, dar.

Es wurde drei Gefäßversuche unter Gewächshausbedingungen mit den erwähnten drei Baumarten in einem nährstoffarmen, sandigen neutralen Boden (ähnelt den ägyptischen Böden) durchgeführt. Dazu wurden die Pflanzen vorkultiviert und dann in größere Gefäße umgesetzt. Den Böden wurden gestaffelte Mengen von in Wasser gelöstem $\mathrm{ZnSO}_{4} 7 \mathrm{H}_{2} \mathrm{O}$, $\mathrm{CdSO}_{4} 8 \mathrm{H}_{2} \mathrm{O}$ und $\mathrm{Pb}\left(\mathrm{NO}_{3}\right)_{2}$ zugegeben. Nach 10 Wochen Wachstumszeit wurden die Sprosse geerntet und folgende Parameter bestimmt: Sprossmasse, Wurzelmasse, Konzentration und Entzug der Sprosse an N, P, K, Ca und Mg sowie die Gehalte der Schwermetalle Zn, Cd und $\mathrm{Pb}$. Zur Beantwortung des 2 Zieles wurden die Böden mit den entsprechenden Mikrobenkulturen geimpft.

\section{Literaturüberblick}

Die Anbauprobleme in Ägypten sind vielfältig wegen Wasserknappheit und schwierigen Wachstumsbedingungen, z.B. jahrelange Trockenheit, hohe Temperaturen, sowie enormer Schwund fruchtbaren Bodens durch Degradation und Winderosion, oft verursacht von Mensch, Tier und Natur. Auch Ägypten trägt dazu bei, dass jährlich weltweit 6 Mio. ha 
(Global, 2000; 1980) Kulturland vernichtet werden. Auch führte die Industrie zu einer Steigerung der Anbauprobleme bei landwirtschaftlichen Kulturen durch steigenden Industrieabfall, aufgrund einer Verringerung der Fruchtbarkeit des Bodens und Verschmutzung des Wassers und der Luft. Deshalb wird eine Erweiterung der Anbaufläche in Industrie- und Entwässerungsgebieten mit anpassungsfähigen Holzbäumen angestrebt. Zur Verwirklichung dieses Zieles werden zahlreiche Forschungsprogramme von den ägyptischen Universitäten und Forschungsinstituten durchgeführt. Die Anpassungsmechanismen der holzliefernden Bäume an die klimatischen und Boden-Stressbedingungen sollen erforscht werden. Dazu gehört die Reaktion dieser Holzbäume auf hohe Schwermetall-Konzentrationen im Boden, die Ermittlung ihrer Toleranzgrenzen für die verschiedenen Schwermetalle sowie die Selektion anpassungsfähiger Bäume.

\section{Schwermetalle}

Schwermetalle sind als natürliche Bestandteile der Gesteine, regional sehr unterschiedlich, in allen Böden enthalten. $\mathrm{Zu}$ den Schwermetallen zählen essentielle Spurennährstoffe wie Kupfer oder Zink, aber auch schädliche Elemente wie Blei oder Cadmium.

Im Zuge der Industrialisierung gelangt bis heute eine stetig zunehmende Menge an Schadstoffen in die Umwelt. Dabei finden vor allem Schwermetallimmissionen eine große Beachtung, weil Schwermetalle, anders als z.B. viele organische Schadstoffe, keinen Abbau und damit auch keine Entgiftung erfahren, sondern sich in der Umwelt, vor allem in Böden, anreichern.

In geringen, meist unschädlichen Konzentrationen sind Schwermetalle schon von Natur aus in allen Böden vorhanden. Daneben kann es lokal durch natürliche Vorgänge zu extremen Schwermetallanreicherungen kommen, z.B. am Ausbiss von erzführenden Gesteinsgängen (Lag et al., 1970; Ernst, 1974). Der weitaus bedeutendere Anteil von Schwermetallanreicherungen in der Umwelt ist jedoch anthropogener Art. Dies kann durch sehr verschiedene Materialien und auf unterschiedlichen Wegen erfolgen, z.B. durch

- Abraummaterialien bergbaulicher Tätigkeit ( Erze, Kohle), die durch Erosionsvorgänge z.T. verfrachtet werden können oder durch schwermetallhaltige Waschwässer, wie sie bei der Erzaufbereitung anfallen und in Flüsse eingeleitet werden (Massey, 1972; Takijma und Katsumi, 1973; Hodenberg, 1974);

- Abwässer, Schlacken sowie gas- und staubförmige Emissionen von Industriebetrieben (Vetter und Mählhop, 1971; Buchauer, 1973); die bei der Verbrennung fossiler Energieträger, Kohle, Öl (Erlenkeuser et al., 1974) und von Müll freiwerdender Gase und Stäube sowie die Schlacken; 
- den Kraftfahrzeugverkehr (Singer und Hanson, 1969; Neumann, 1980) ;

- die Ausbringung von schwermetallhaltigen Pflanzenschutzmitteln und z.T. auch von Mineraldüngern (Rieder und Schwertmann, 1973; Stenström und Vahter, 1974);

- Einsatz von Siedlungsabfällen (Klärschlämme, Müllkomposte) als Bodenverbesserungs- und Düngemittel (Haan, 1972; Kick, 1974; Laurenz, 1978).

\section{Elemente $\mathrm{Zn}, \mathrm{Cd}$ und $\mathrm{Pb}$}

Im Folgenden werden die in dieser Arbeit behandelten Schwermetalle Zink, Cadmium und Blei kurz charakterisiert (nach Kabata-Pendias und Pendias, 1992).

Zink: Meist kommt es als zweiwertiges Kation $\left(\mathrm{Zn}^{2+}\right)$ vor. Es zeigt große Affinität $\mathrm{zu} \mathrm{H}_{2} \mathrm{~S}$, und Phosphat, sowie verschiedenen organischen Komponenten und bildet stabile Komplexe. Es ist relativ mobil im Boden und wird meist in den Oberflächenhorizonten akkumuliert. Viele Untersuchungen (Arriechi und Ramirez, 1997, Mandal und Hazra,1997) zeigten, dass die Zinkadsorption stark vom Gehalt an Ton und organischer Substanz im Boden abhängig ist. Zink ist über einen weiten $\mathrm{pH}$-Bereich relativ leicht pflanzenverfügbar, und zählt in geringen Konzentrationen im Boden $\mathrm{zu}$ den Mikronährstoffen bzw. zu den Hochleistungselementen.

Cadmium: Im Boden kommt es meist als zweiwertiges Kation $\left(\mathrm{Cd}^{2+}\right)$ vor, bildet aber auch komplexe Ionen wie z.B. $\mathrm{CdCl}^{+}, \mathrm{CdOH}^{+}, \mathrm{CdHCO}_{3}{ }^{+}, \mathrm{CdCl}_{3}^{-}$, etc. und Chelatkomplexe mit organischen Anionen. Cadmium ist recht mobil im Boden. Der wichtigste Faktor für die Mobilität der Cd-Ionen ist der $\mathrm{pH}$-Wert (Anderson und Nilsson, 1974). Weiter sind organische Substanzen sowie Fe- und Mn-Oxide von Bedeutung für die Cd-Bindung. Am mobilsten ist Cadmium bei einem niedrigen $\mathrm{pH}$-Wert. Cadmium wird meist effektiv von Pflanzen aufgenommen. Der größte Teil des aufgenommen Cadmium wird in den Wurzeln akkumuliert, wobei es aber auch innerhalb der Pflanze mobil ist und häufig in Samen und Früchte verlagert wird. Es ist ein hoch toxisches Element für alle Organismen. Die große Gefahr liegt in seiner starken Affinität zu schwefelhaltigen Gruppen verschiedener Enzymkomponenten und zu Seitenketten von Proteinen und zu Phosphatgruppen.

Blei: Es kommt im Boden vorwiegend als zwei-oder vierwertiges Kation $\left(\mathrm{Pb}^{2+}\right.$ und $\left.\mathrm{Pb}^{4+}\right)$ vor, wobei es mit organischen Komponenten (Huminstoffen), sowie mit mineralischen Anteilen (Tonmineralien, Mn-Oxiden, Fe-, Al-Hydrooxiden) stabile Komplexe bildet. Lucanbouche et al. (1997) bemerkten, dass Blei auch relativ stark an Sand adsorbiert wird. Im Boden ist Blei daher relativ fest gebunden (Aten und Gupta, 1996) und eher immobil. Im charakteristischen 
vertikalen Verteilungsmuster, nehmen die $\mathrm{Pb}$-Gesamtgehalte mit der Tiefe deutlich ab. Die Löslichkeit des Elements steigt bei pH-Werten unter 6. Die Bleiaufnahme in Pflanzen erfolgt häufig über die Oberfläche als atmosphärische Partikel oder durch Reususpension. Innerhalb der Pflanze ist Blei nur schwer verlagerbar, es verbleibt vor allem in den Wurzeln oder im Spross, wenn es über diesen aufgenommen wird. Blei ist als Schadstoff für alle Organismen bekannt.

\section{Einfluss der Pflanzen auf die Schwermetallaufnahme}

Neben den die Pflanzenverfügbarkeit der Schwermetalle beeinflussenden Bodenfaktoren spielen auch die Pflanzen selbst eine große Rolle hinsichtlich der Schwermetallaufnahme. Von allgemeiner Bedeutung ist die Pflanzenart (Grejtovsky und Pirc, 2000) und die Pflanzen-Morphologie, d.h. die Oberflächenbeschaffenheit, Form und Stellung der Früchte und Blätter sowie das Oberflächen-Volumenverhältnis. So weisen beispielsweise Äpfel und Kartoffeln mit einem engen Oberflächen-Volumenverhältnis niedrigere $\mathrm{Pb}$-Gehalte auf als Pflanzen mit einem mittleren (Lauch und Kopfsalat) und solche mit einem weiten Verhältnis wie Feldsalat und Petersilie (Schoemakers, 1986).

Auch die wurzelmorphologischen Eigenschaften beeinflussen die Schwermetallaufnahme. So können Pflanzen mit großem und feinverteiltem Wurzelwerk wie Cruciferen wesentlich stärker Schwermetalle aufnehmen als beispielsweise Gramineen (Crößmann, 1982). Die Anreicherung in der Pflanze ist ebenso abhängig vom Pflanzenalter bzw. vom jeweiligen Entwicklungsstadium der Pflanzen sowie von der Vegetationsdauer. Darüber hinaus wird die Schwermetall-Aufnahme von der Wurzeldichte sowie von der Infektion der Wurzeln mit symbiontischen (V)A-Mykorrhiza-Pilzen wesentlich beeinflusst (Fabig, 1982; Joner und Leyval, 2001). Cieslinski et al. (1996) berichteten über die Notwendigkeit der Erforschung des Bioakkumulation bzw. des Umwelt-Zyklus von Cd für das Management von landwirtschaftlichen Böden und Kulturen unter dem Einfluss dieses toxischen Schwermetalls.

Foroughi et al. (1981) erklären den höheren Schwermetallgehalt in der Bohnenfrucht gegenüber dem der Tomate mit den kürzeren Transportwegen von der Wurzel bis zur Frucht bei der Bohne. Außerdem nehmen Tomatenfrüchte nicht aktiv an der Transpiration teil, da sie keine Somata besitzen.

Die Artspezifität der Schwermetallaufnahme zeigt sich sehr deutlich an verschiedenen Gramineen. In untersuchten Weizenproben war die Menge an $\mathrm{Pb}$ und $\mathrm{Cd}$ etwa gleich groß, wohingegen beim Roggen der Pb-Gehalt fünf- bis sechsmal höher war als der Cd-Gehalt. Auch Horak (1976) fand die höchsten Cd-Gehalte in Weizenkörnern. Sommer (1979) 
ermittelte bei den Getreidearten zunehmende Cd-Gehalte in der Reihenfolge Gerste, Hafer, Weizen.

Selbst bei den verschiedenen Sorten einer Pflanzenart können sich die Schwermetalle in unterschiedlichem Maße anreichern. Rosopulo und Diez (1982) konnten einen gewissen Unterschied für die Elemente $\mathrm{Cd}, \mathrm{Zn}$ und $\mathrm{Cu}$ bei zehn untersuchten Weizensorten nachweisen. Ein deutlicher Unterschied bestand zwischen den Sorten 'Aquila' mit dem niedrigsten und 'Disponent' mit dem höchsten Gehalt an Cd. Auch Page (zitiert in Kloke, 1981) berichtete über eine sorten- und sogar zuchtlinienspezifische Schwermetallaufnahme bei Mais, Salat und Sojabohnen, während Brüne et al. (1982) für Cd bei Spinat, Salat, Bohnenkraut, Karottenkraut und Karotten nur geringfügige Sortenunterschiede feststellten. Innerhalb einer Pflanze finden sich keine gleichmäßigen Schwermetallkonzentrationen. In den verschiedenen Organen einer Pflanze sind die Schwermetallkonzentrationen unterschiedlich. So fanden Rosopulo und Diez (1982) bei Getreidearten höhere Cd-Konzentrationen in den vegetativen Teilen, wobei Hafer-, Winterweizen- und Wintergerstenstroh ein etwa gleich großes Anreicherungsvermögen für $\mathrm{Cd}$ aufwiesen, während dieses bei Sommergersten- und Roggenstroh wesentlich geringer war. Maisstroh reichert $\mathrm{Cd}$ besonders stark an. Kampe (1980) berichtete über bis zu 25-fach höhere Cd-Konzentrationen im Kraut bzw. Stroh von Möhren, Tomaten und Hafer gegenüber den Früchten bzw. Körnern und Wurzeln.

Venter (1980) untersuchte verschiedene Gemüsearten und fand für $\mathrm{Zn}$ und $\mathrm{Cd}$ die höchsten Schwermetallgehalte in den Blättern. Eine Mittelstellung nahmen die Knollen und Wurzeln ein, während in den Früchten die Gehalte wesentlich niedriger lagen. Allerdings lässt sich nicht verallgemeinern, dass vegetative Pflanzenorgane generell höhere Schwermetallgehalte aufweisen als generative, weil der Gehalt in der Pflanze vom Gehalt im Boden abhängig ist und die Aufnahme von Pflanze zu Pflanze unterschiedlich und auch elementspezifisch ist. $\mathrm{Zu}$ ähnlichen Ergebnissen kamen Badri und Springuel (1994). Sie fanden, dass die höchsten Werte von Co und Ni in Cleome droserifolia Pflanzen akkumuliert wurden.

\section{Einfluss der Bodenfaktoren auf die Schwermetallaufnahme}

Entscheidend für die Schwermetallaufnahme durch die Pflanzen ist nicht der Gesamtgehalt eines Bodens an Schwermetallen, sondern der pflanzenverfügbare Anteil. Dieser ist von einer Vielzahl von Bodenfaktoren abhängig. Eine große Rolle spielen beispielsweise der pH-Wert, der Tongehalt, der Gehalt des Bodens an organischer Substanz, die Sesquioxide (Eisen- und Aluminiumoxide und-hydroxide), die Redoxverhältnisse u.a. Die Tonminerale, die Huminstoffe und die Sesquioxide bestimmen weitgehend die Adsorptions-und Pufferkapazität eines 
Bodens (Sticher, 1980). Diese Bestandteile sind in der Lage, Schwermetalle adsorptiv zu binden und damit aus der ökologisch relevanten Lösungsphase zu entfernen. Je höher etwa der Tongehalt, der Gehalt an organischer Substanz oder der Gehalt an Sesquioxiden ist, um so besser ist der Boden in der Lage, zugeführte Schwermetalle zu sorbieren.

Von großer Bedeutung für die Pflanzenverfügbarkeit der Schwermetalle ist der $\mathrm{pH}$-Wert. Die Löslichkeit wird stark vom pH-Wert, den Redoxbedingungen und der Menge an Sorptionsträgern beeinflusst (Herms und Brümmer, 1984; Chuan et al., 1996; Dudka et al., 1996). Mit ansteigendem Boden-pH verringert sich die Löslichkeit der meisten Schwermetalle. Daher ist ihre Konzentration in alkalischen und neutralen Böden niedriger als in sauren Böden. Bei niedrigem $\mathrm{pH}$ werden dagegen viele Elemente (wie $\mathrm{Zn}$ und $\mathrm{Cd}$ ) sogar ausgewaschen. So weisen nach Herms und Brümmer (1980) $\mathrm{Zn}$ und $\mathrm{Cd}$ bei $\mathrm{pH} 7$ und 8 nur eine geringe Löslichkeit auf, die sich mit abnehmendem $\mathrm{pH}$-Wert jedoch sehr stark erhöht. Auch die Gehalte von $\mathrm{N}$ in der Bodenlösung stiegen mit abnehmendem pH-Wert deutlich, jedoch schwächer als bei $\mathrm{Zn}$ und $\mathrm{Cd}$. Der Gesamtgehalt der Elemente spielt insofern eine Rolle, als bei gleichem $\mathrm{pH}$-Wert die Löslichkeit mit steigendem Gesamtgehalt zunimmt. $\mathrm{Cu}$ und $\mathrm{Pb}$ weisen erst bei $\mathrm{pH}-$ Werten unter 4 und 5 steigende Konzentrationen in der Bodenlösung auf. Bei beiden Elementen liegt bei pH-Werten von 5 bis 6 ein Minimum der Löslichkeit, während die Mobilisierung von $\mathrm{Pb}$ und vor allem $\mathrm{Cu}$ bei pH-Werten von 6 bis 8 wieder zunimmt. Das führen Herms und Brümmer (1978) auf die in diesem pH-Bereich erhöhte Löslichkeit von organischen Komplexbildnern zurück. Der Einfluss der Bodenreaktion auf die Schwermetall-Löslichkeit ist nach diesen Ergebnissen bei $\mathrm{Zn}$ und $\mathrm{Cd}$ am größten, gefolgt von $\mathrm{Ni}, \mathrm{Cu}$ und $\mathrm{Pb}$. Allerdings können unter reduzierenden Bedingungen auch bei $\mathrm{pH}-$ Werten über 7 die Schwermetalle $\mathrm{Cd}, \mathrm{Zn}$ und $\mathrm{Pb}$ verstärkt in Lösung gehen, da unter diesen Bedingungen eine erhöhte Mobilisierung durch organische Komplexbildner stattfindet. Bei pH-Werten unter 7 sind die Löslichkeiten der Schwermetalle im anaeroben Milieu jedoch stark verringert (Herms und Brümmer, 1978).

\section{Einfluss der Schwermetalle auf die Aufnahme anderer Elemente}

Durch Schwermetalle wird die Aufnahme anderer Elemente beeinflusst. Foroughi et al. (1976) berichteten, dass durch $\mathrm{Cd}$ der Fe-Gehalt in allen oberirdischen Pflanzenteilen von Tomaten gesenkt, in der Wurzel aber erhöht wurde. Der Fe-Transport in die oberirdischen Pflanzenorgane wird offensichtlich gehemmt. Zn behindert den Fe- Transport aus der Wurzel in die oberirdischen Teile in derselben Weise wie Cd (Foroughi et al., 1982). Die P- und KGehalte der Pflanze werden durch die Schwermetallaufnahme ebenfalls negativ beeinflusst 
(Foroughi et al., 1979). Foroughi et al. (1982) untersuchten Buschbohnen und fanden, dass $\mathrm{Ni}$ und $\mathrm{Cr}$ die Verteilung der übrigen Elemente nur wenig beeinflusst. Durch hohe $\mathrm{Pb}-\mathrm{Gaben}$ wurde die $\mathrm{N}$ - und $\mathrm{Mg}$-Verteilung beeinflusst. Durch Zn- und $\mathrm{Cd}-\mathrm{Zufuhr}$ wurde der Fe-Gehalt und der Mn-Gehalt in den oberirdischen Pflanzenteilen vermindert. Eine Cd-Zufuhr führte zu einer verminderten P-Aufnahme in Wurzeln, Blättern und Früchten ebenso wie eine erhöhte Zn-Gabe.

\subsection{Anmerkungen zu den wichtigen holzliefernden Bäumen in Ägypten:}

Acacia saligna (Fam. Leguminosae)

Dieser Baumart stammt aus Australien und wurde um 1840 in Nordafrika eingeführt. Der Baum wächst relativ schnell und erreicht eine Höhe bis $8 \mathrm{~m}$ und einen Durchmesser bis $30 \mathrm{~cm}$. A. saligna bilden im Frühling gelbe Blüten (NAS, 1980a).

Die Samen sind dunkelbraun bis schwarz. Ein kg Samen enthält 14.000 - 25.000 Samen. Das Blatt ist dunkelgrün bis blaugrün und 8-25 cm lang (Maslin, 1974). Der Baum kann mit Samen (nach $\mathrm{H}_{2} \mathrm{SO}_{4}$-Behandlung) und Stängelstecklingen vermehrt werden (NAS, 1980a). A. saligna kann Temperaturen bis $36^{\circ} \mathrm{C}$, Trockenheit, Frost, sandige, alkalische bis tonreiche Böden und Bodenversalzung tolerieren (Hall und Turnbull, 1976; NAS, 1980a; Simmons, 1981).

Junge Sprosse und Blätter werden als Futter (Michaelides, 1979; NAS, 1980a; El-Lakany in Turnbull, 1987). Der Stamm dient der Produktion von Möbeln oder Brennholz. A. saligna Bäume werden darüber hinaus als Schutzstreifen gegen Wind bzw. Winderosion sowie als Zierbäume angebaut.

\section{Casuarina equisetifolia (Fam. Casuarinaceae)}

Diese Baumart stammt aus den Tropen und Subtropen Australiens und Afrikas. Ihre Bäume wachsen langsam, können aber eine Höhe von $30 \mathrm{~m}$ und einen Durchmesser von $2 \mathrm{~m}$ erreichen. Die Samen sind sehr klein; 1 kg enthält 660.000 - 990.000 Samen, welche für die Vermehrung der Bäume verwendet werden. Das Blatt ist dunkelgrün und 5-10 cm lang. Casuarina-Bäume tolerieren Trockenheit, Bodenversalzung und Frost.

Sie wachsen sehr gut auf sandigen und sogar auf Granitböden (NAS, 1983b). In Ägypten werden diese Bäume als Schutzstreifen gegen Wind bzw. Winderosion sowie auf versalzten und armen Böden angebaut. In den letzten Jahren werden sie darüber hinaus verstärkt auf kontaminierten Böden für den Umweltschutz in Industriegebieten kultiviert. In tropischen Regionen, z.B. in den Philippinen, werden Casuarina-Bäume wegen ihrer Fähigkeit der №Fixierung zwischen Kakao-Bäumen zur Verbesserung des N-Status des Bodens angebaut 
(Aspiras, 1981). In Graslandschaften sind sie auch weit verbreitet (NAS, 1983b). Diese Bäume werden oft zur Herstellung von Möbeln, Brennstoffen, Rudern, Wasserrädern und Holzkohle sowie im Schiffsbau verwendet.

\section{Cupressus sempervirens (Fam. Cupressaceae)}

Cupressus sempervirens gehört a jenen Wirtschaftsholzarten, die sich für Aufforstungen auf Kalk- und Tonböden sowie auf trockenen Standorten infolge hoher Anpassungsfähigkeit und Dürretoleranz gut bewährt haben. Seit Jahrhunderten wird Cupressus mit Erfolg für Windschutzstreifen verwendet. In einigen für kalte Winde exponierten Lagen ist es ohne den Anbau schützender Cupressus-Reihen unmöglich, Obstplantagen zu betreiben.

Das Schwergewicht des natürlichen Areals liegt im östlichen Mittelmeergebiet. Es schließt Griechenland, die Ägäischen Inseln, Kreta, Zypern, Syrien, Israel, Sizilien sowie den Libanon ein und reicht darüber hinaus bis in den Südwesten des Iran. In diesem weitläufigen Gebiet wächst die Art sowohl in Rein- wie in Mischbeständen: in Syrien und Libanon u.a. in Mischung mit Pinus brutia, in Anatolien (Türkei) mit P. brutia, P. nigra oder Cedrus libani. C. sempervirens ist als spontane oder subspontane oder eingeführte Art in mediterranen Ländern wie Griechenland, Italien, Frankreich und Spanien weit verbreitet. In Italien stellt die Toskana das Zentrum des Anbaus dar. Dort wird C. sempervirens in großem Umfang sowohl als Waldbaum wie als Ziergehölz kultiviert und ist keineswegs nur auf Friedhöfen zu finden.

Während des langen, von Süd nach Nord fortschreitenden Einbürgerungsprozesses im Mittelmeerraum scheint ein gewisser Selektionsdruck in Richtung Kälteresistenz stattgefunden zu haben (Radd et al., 1990).

In natürlichen Beständen, so z.B. in der Cyreniaka (Libyen, $400 \mathrm{~m}$ ü. NN) kann die Art Höhen von 30-35 m und Durchmesser von 80-100 cm erreichen.

Die Krone von Cupressus ist durch ein dunkles Grün gekennzeichnet, welches nur im Frühjahr beim Erscheinen der jungen Triebe und der männlichen Blüten gemustert aussieht.

Die Stämme von Cupressus sind sehr intensiv beastet. Die Stämme alter Cupressus-Bäume schließen normalerweise mit einer dünnen, faserigen, dunkelgrauen Borke $a b$, welche von Jugend an durch feine Längsstrukturen gekennzeichnet ist. Das sehr homogene Holz ist von feiner Textur, der Faserverlauf aber nicht immer gleichmäßig. Das Holz der MittelmeerCupressus ist schwer.

Die gewöhnlich schuppenförmigen Blattorgane der Cupressus-Bäume stehen dekussiert gegenständig am Spross, liegen diesem dicht an und überdecken sich dachziegelartig. Die 
basalen Teile der Blätter können mit den Zweigen verwachsen. Im zweiten Jahr trocknen die Blätter mit fortschreitender Entwicklung des Spross-Periderms aus und fallen ein Jahr später ab.

Die Art C. sempervirens ist eine monözische Art, deren 3-5 mm lange und ca. $2 \mathrm{~mm}$ breite gelbe bis gelbbraune männliche Blüten oft in großer Zahl an der Spitze von Kurztrieben stehen. Meist sind sie schon im Winter zu erkennen und kommen vor allem im unteren Teil der Krone vor. Die weiblichen Zapfenblüten sind von kugeliger Gestalt (2,5 mm), von grünlicher Farbe, setzen sich aus 4 bis 7 Samenschuppen mit je 6 bis 20 Samenanlagen zusammen und kommen bevorzugt im oberen Teil der Krone vor. Generell blüht die Art von Januar bis Februar. Die Samen sind 5-6 mm lang und 3-4 breit. Das Tausendkorngewicht beträgt 6- $8 \mathrm{~g}$.

Der Cupressus-Baum gedeiht unter etwa gleichen Klimabedingungen wie der Ölbaum (Olea europaea). Pavari (1934) umgrenzt ihr Vorkommen mit den Klimadaten des Lauretums und des klassischen Castanetums. Dem entsprechen: mittlere Jahrestemperaturen von $15-23^{\circ} \mathrm{C}$ und ein Temperaturminimum von $-12^{\circ} \mathrm{C}$. C. sempervirens durchläuft eine Winterruhe. Larcher (1975) berichtete über Frostschäden bereits bei $-14^{\circ} \mathrm{C}$, Puric (1967) fand hingegen, dass alte Bäume noch Temperaturen von $-20^{\circ} \mathrm{C}$ ohne Schäden überstehen. Die Cupressus-Bäume wachsen unter verschiedenen Bodenbedingungen. Größere Bestände werden häufig auf Kalkund Mergelstandorten begründet, die zu nährstoffarmen, flachgründigen, trockenen Böden verwittern. C. sempervirens gedeiht auf sauren wie auf alkalischen Substraten (pH 5,8-8,2). Nachteilig wirkt sich aus, dass C. sempervirens kaum den Boden verbessert, denn die Anreicherung organischer Substanzen und das Aufkommen einer Bodenflora vollziehen sich sehr langsam. Aus diesem Grund wird empfohlen, C. sempervirens bei der Aufforstung vegetationsloser oder degradierter Flächen nur in Mischung mit Kiefern und Laubbäumen zu verwenden (Giordano, 1979). C. sempervirens kann mit Samen vermehrt werden.

Wie frühere Autoren berichteten, war diese Art einst der wichtigste Waldbaum im östlichen Mittelmeergebiet. Nach den Überlieferungen war die erste Flotte des Kreterkönigs Minos aus Cupressusholz gebaut. Gleiches gilt für Schiffe aus Ägypten, Palästina und Phönizien. Cupressus-Bäume werden in zahlreichen Kunstwerken mehrerer Epochen dargestellt. Als medizinisch wirksame Komponenten werden genannt: Tannine, Terpene, Kohlenwasserstoffe, Alkohole, Ketone, Ester sowie Furfurol. In Frankreich stellen 18 Laboratorien etwa 20 auf Cupressusöl basierende pharmazeutische Präparate her. Cupressusöl wird vorzugsweise für die Herstellung von Herren-Parfüm verwendet. 


\section{Material und Methodik}

\subsection{Boden und Bodenvorbereitung}

Für alle Versuche wurde eine Bodenmischung aus Schoninger-Boden ( $\mathrm{pH}$ in $0,01 \mathrm{M} \mathrm{CaCl}_{2}=$ 6,1; $78 \%$ Sand, $15 \%$ Schluff und 7,2 \% Ton) und Quarzsand (beide gesiebt auf $2 \mathrm{~mm}$ ) im Verhältnis 2:1 verwendet. Die Bodenmischung wurde mit $180 \mathrm{mg} \mathrm{CaCO}_{3} / \mathrm{kg}$ auf einen $\mathrm{pH}-$ Wert von 7-7,1 aufgekalkt, um einen ähnlich nährstoffarmen, neutralen Boden, wie unter ägyptischen Bedingungen, zu erhalten. Die an der Luft getrocknete Bodenmischung wurde dann mit Monocalciumphosphat (MCP) entsprechend $90 \mathrm{~kg} / \mathrm{ha} \quad(=122,1 \mathrm{mg} \mathrm{MCP} / \mathrm{kg})$ gedüngt. Anschließend wurde der Boden in $2 \mathrm{~kg}$ Versuchstöpfe gefüllt.

Tab.1: Chemische Kenndaten des Versuchsbodens (Bodenmischung aus Schoninger-Boden und Quarzsand mit $180 \mathrm{mg} \mathrm{CaCO} / / \mathrm{kg}$ aufgekalkt)

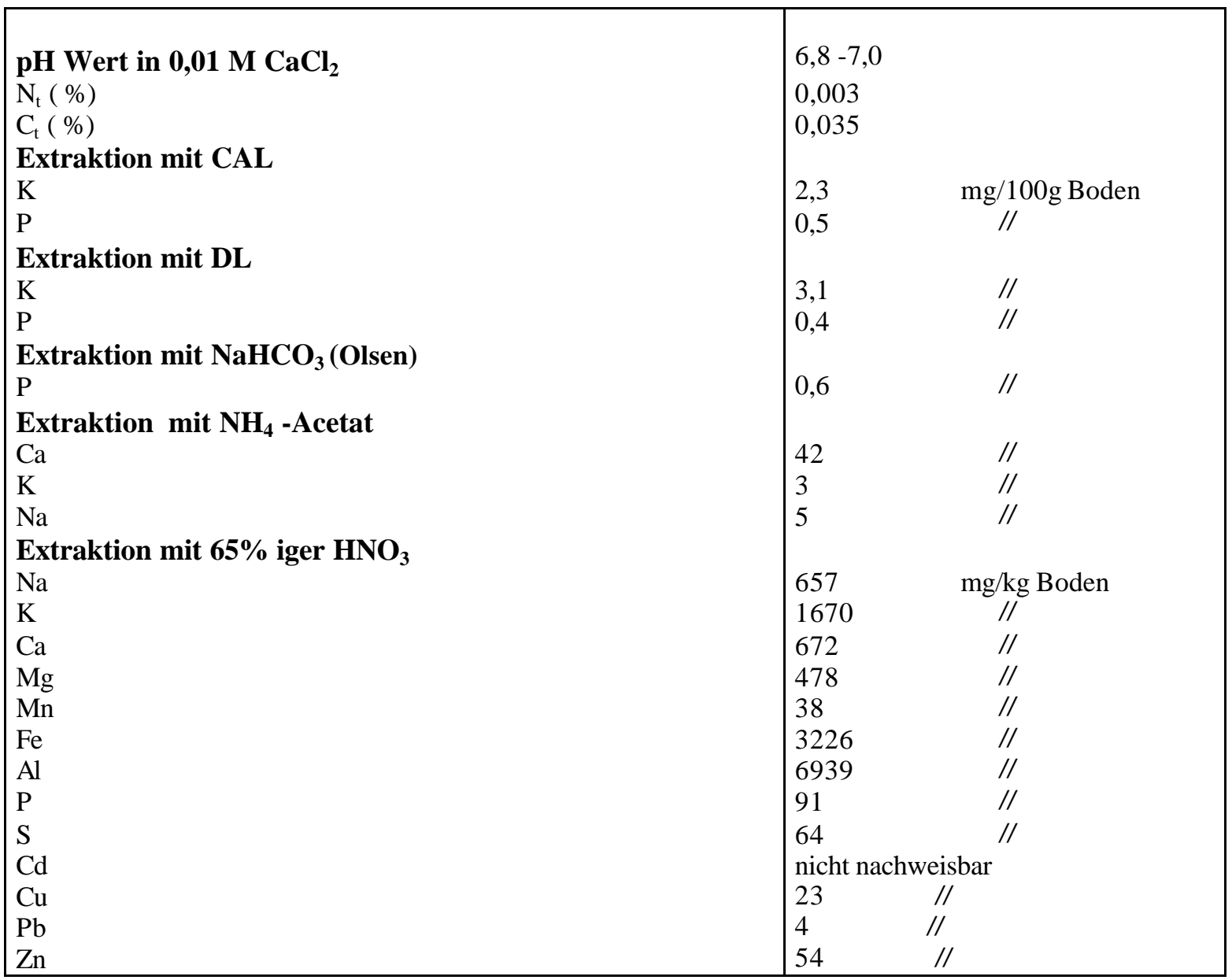

\subsection{Versuchsräume und Gefäße}

Die Versuche wurden in Gewächshauskabinen durchgeführt. Eine automatische TemperaturSteuerung regelte die Kabinentemperatur auf mindestens $22^{\circ} \mathrm{C}$ während der Nacht bis $28^{\circ} \mathrm{C}$ am Tage. Die Höchsttemperaturen hingen von der Witterung ab und schwankten entsprechend, bei starker Sonneneinstrahlung wurden maximal $38^{\circ} \mathrm{C}$ erreicht. Die relative 
Luftfeuchtigkeit lag zwischen $40 \%$ und $75 \%$. An bewölkten Tagen wurde mit HPL-Lampen (Philips HP-N, 400 W, Typ 557221, G/74) zusätzlich beleuchtet. Wie schon erwähnt, wurden 2 kg-Töpfe (aus schwarzem Plastik) verwendet. Tab. 1a gibt als Beispiel die mittlere MessWerte für das Jahr 1998 an.

Tab.1a: Beispiel für die Verläufe den mittleren wöchentlichen Tages- und Nacht-Temperaturen sowie relative Luftfeuchtigkeiten (min. und max.) in der Vegetationszeit von Mai bis Dezember 1998

\begin{tabular}{|c|c|c|c|c|c|c|c|}
\hline \multirow[t]{2}{*}{ Jahr } & \multirow[t]{2}{*}{ Monate } & \multicolumn{2}{|c|}{ Woche } & \multicolumn{2}{|c|}{ Temperatur ${ }^{\circ} \mathrm{C}$} & \multicolumn{2}{|c|}{ relative Luftfeucht. \% } \\
\hline & & & & Tag & Nacht & $\min . \pm 5 \%$ & $\max . \pm 5 \%$ \\
\hline & Mai & 28.05 & -31.05 & 33 & 21 & 45 & 80 \\
\hline & & 01.06 & -07.06 & 33 & 21 & 45 & 80 \\
\hline & & 08.06 & -15.06 & 30 & 20 & 45 & 80 \\
\hline & & 16.06 & -22.06 & 30 & 22 & 40 & 85 \\
\hline & Juni & 23.06 & -28.06 & 33 & 23 & 40 & 85 \\
\hline & & 29.06 & -05.07 & 28 & 22 & 50 & 85 \\
\hline & & 06.07 & -12.07 & 28 & 22 & 50 & 85 \\
\hline & & 13.07 & -19.07 & 28 & 22 & 45 & 80 \\
\hline & Juli & 20.07 & -26.07 & 33 & 21 & 40 & 80 \\
\hline & & 27.07 & -02.08 & 28 & 22 & 45 & 85 \\
\hline & & 03.08 & -09.08 & 35 & 23 & 35 & 75 \\
\hline & & 10.08 & -16.08 & 35 & 23 & 35 & 75 \\
\hline & & 17.08 & -23.08 & 33 & 23 & 40 & 80 \\
\hline & August & 24.08 & -30.08 & 28 & 22 & 50 & 75 \\
\hline & & 31.08 & -06.09 & 28 & 22 & 60 & 90 \\
\hline & & 07.09 & -13.09 & 30 & 22 & 45 & 80 \\
\hline & & 14.09 & -20.09 & 30 & 23 & 45 & 75 \\
\hline \multirow{14}{*}{1998} & Sentember & 21.09 & -27.09 & 33 & 23 & 45 & 80 \\
\hline & & 28.09 & -04.10 & 28 & 23 & 50 & 75 \\
\hline & & 05.10 & -11.10 & 28 & 22 & 50 & 65 \\
\hline & & 12.10 & -18.10 & 28 & 21 & 45 & 65 \\
\hline & Oktober & 19.10 & -25.10 & 28 & 22 & 40 & 65 \\
\hline & & 26.10 & -01.11 & 28 & 21 & 45 & 60 \\
\hline & & 02.11 & -08.11 & 28 & 20 & 45 & 60 \\
\hline & & 09.11 & -15.11 & 25 & 22 & 50 & 60 \\
\hline & & 16.11 & -22.11 & 26 & 20 & 35 & 55 \\
\hline & November & 23.11 & -29.11 & 28 & 20 & 30 & 40 \\
\hline & & 30.11 & -06.12 & 28 & 22 & 25 & 30 \\
\hline & & 07.12 & -13.12 & 28 & 22 & 25 & 35 \\
\hline & & 14.12 & -20.12 & 28 & 21 & 35 & 45 \\
\hline & Dezember & 21.12 & -27.12 & 28 & 22 & 35 & 45 \\
\hline MW & & & & 29,56 & 21,8 & 42,3 & 69,5 \\
\hline
\end{tabular}




\subsection{Versuchspflanzen und Pflanzenzucht}

Aus Ägypten stammende Samen von Acacia saligna, Casuarina equisetifolia und Cupressus sempervirens wurden verwendet (Tab.2). Wegen der Hartschaligkeit der Samen von A. saligna, wurde versucht, die Keimung zu beschleunigen. Deshalb wurden die Samen mit konz. $\mathrm{H}_{2} \mathrm{SO}_{4}$ für 105 min behandelt. Nach dieser Behandlung keimten die AcaciaSamen schon nach 8 Tagen. Bei den anderen beiden Baumarten wurden die Samen im Wasser für 120 min eingeweicht und dann in Anzuchtsschalen (11 kg Fassungsvermögen) gesät. Im allgemeinen zeigten die Sämlinge ein relativ langsames und wegen Unreinheit der Samen ungleiches Wachstum. Während der Anzuchtdauer von 2 Monaten wurde einmal mit Volldünger $($ Wuxal $=4 \mathrm{ml} / \mathrm{l})$ gedüngt. Nach dieser Anzuchtperiode wurden die Sämlinge in 1/3 kg-Töpfe (aus schwarzem Plastik) umgepflanzt (1 Pflanze/Topf bei A. saligna und C. sempervirens und 2 Pflanzen/Topf bei $C$. equiseitfolia). Der Boden für dieser kleinen Töpfe wurde einmalig mit einer Start-N-Düngung, entsprechend $30 \mathrm{~kg} \mathrm{~N} / \mathrm{ha}(=28,56 \mathrm{mg}$ $\mathrm{NH}_{4} \mathrm{NO}_{3} / \mathrm{kg}$ Boden) und später mit einer $\mathrm{N}$-freien Nährlösung mit $10 \mathrm{ml} / 1 / 3 \mathrm{~kg}$ Topf (30 ml/kg Boden) (Tab.3) gedüngt. In diesen Töpfen wuchsen die Pflanzen weitere 4 Monate. Anschließend wurden sie in die $2 \mathrm{~kg}$ Versuchstöpfe umgepflanzt. Die Sämlinge der drei Baumarten hatten in allen Versuchen zum Zeitpunkt der Schwermetall-Behandlung das gleiche Alter von 7 Monaten. Sie wurden, je nach Baumart, nach gleicher Größe und gleichen morphologischen Eigenschaften selektiert.

Tab. 2: Versuchspflanzen

\begin{tabular}{|l|l|l|}
\hline Familie & Baumart & Herkunft $*$ \\
\hline Leguminosae & Acacia saligna & Ägypten \\
\hline Casuarinaceae & Casuarina equisetifolia & Ägypten \\
\hline Cupressaceae & Cupressus sempervirens & Ägypten \\
\hline
\end{tabular}

* Es war nicht möglich, zertifizierte Samen zu erhalten 
Beim Umpflanzen wurde darauf geachtet, dass der verwendete Boden ausreichend feucht war, um eine Verletzung der empfindlichen Jungpflanzen $\mathrm{zu}$ vermeiden. Um anhaftendes Bodenmaterial zu entfernen, wurden die Wurzeln vor dem Einpflanzen abgespült.

\subsection{Düngung}

Während der Pflanzenanzucht in Schalen und anschließend in 1/3 kg Töpfen wurden die Versuchspflanzen mit einer $\mathrm{N}$-freien Nährlösung gedüngt (Tab.3). Die dafür angesetzte Stammlösung wurde kühl und dunkel gelagert. Bei der Anwendung wurde die konzentrierte Stammlösung auf das 10-fache mit demineralisiertem Wasser verdünnt. Je 21 der Lösung 1-4 und $200 \mathrm{ml}$ der Lösung 5 und 6 wurden mit demineralisiertem Wasser bis auf 501 aufgefüllt.

\section{Tab. 3: Zusammensetzung der N-freien Nährlösung}

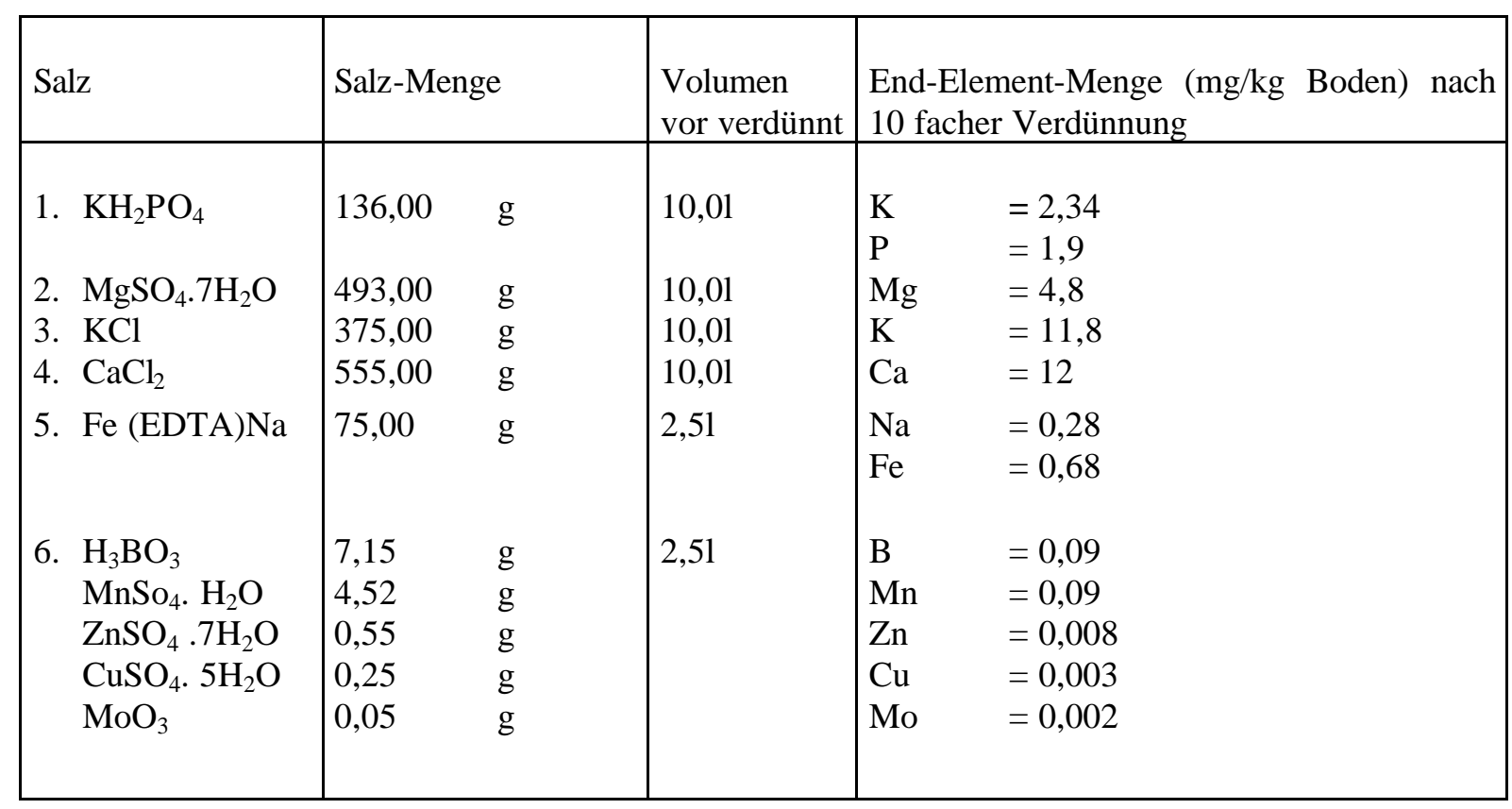

Das Phosphat $\left(\mathrm{Ca}\left(\mathrm{H}_{2} \mathrm{PO}_{4}\right)_{2}\right.$. $\left.\mathrm{H}_{2} \mathrm{O}\right)$ wurde in Pulverform dem trockenen Boden beigemischt (Tab.4).

Nach dem Umpflanzen in $2 \mathrm{~kg}$-Töpfe wurden die Pflanzen mit Stickstoff $\left(\mathrm{NH}_{4} \mathrm{NO}_{3}\right)$, Kalium $\left(\mathrm{K}_{2} \mathrm{SO}_{4}\right)$ und Magnesium $\left(\mathrm{MgSO}_{4} \cdot 7 \mathrm{H}_{2} \mathrm{O}\right)$ in flüssiger Form gedüngt. 
Tab. 4: Die verwendeten Nährstoff-Mengen

\begin{tabular}{|l|l|l|l|}
\hline Düngerform & mg Nährstoff/kg Boden & mg Dünger/kg Boden & kg Reinnährstoff/ha \\
\hline $\mathrm{NH}_{4} \mathrm{NO}_{3}$ & $\mathrm{~N}=67$ & 190,4 & $=200 \mathrm{~N}$ \\
\hline $\mathrm{Ca}\left(\mathrm{H}_{2} \mathrm{PO}_{4}\right)_{2} \cdot \mathrm{H}_{2} \mathrm{O}$ & $\mathrm{P}=30$ & 122,1 & $=90 \mathrm{P}$ \\
\hline $\mathrm{K}_{2} \mathrm{SO}_{4}$ & $\mathrm{~K}=27$ & 59,4 & $=80 \mathrm{~K}$ \\
\hline $\mathrm{MgSO}_{4} \cdot 7 \mathrm{H}_{2} \mathrm{O}$ & $\mathrm{Mg}=13,3$ & 135,2 & $=40 \mathrm{Mg}$ \\
\hline
\end{tabular}

Die N-Düngung wurde bei $A$. saligna und $C$. equisetifolia (in den Versuchen ohne Beimpfung mit Rhizobien bzw. Frankia) zu zwei Terminen (vor und nach der Schwermetall-Behandlung) und bei C. sempervirens zu drei Terminen (zweimal vor und einmal nach der Schwermetall-Behandlung) verabreicht. In Versuch 3 wurde, abgesehen vom $\mathbf{N}$, wie in Tab.4 angegeben, gedüngt.

Die berechneten Werte beziehen sich auf eine Bodentiefe von $20 \mathrm{~cm}$ und eine Bodendichte von $1,5 \mathrm{~g} / \mathrm{cm}^{3}$ (1 ha $=3000.000 \mathrm{~kg}$ Boden $)$.

\subsection{Bewässerung}

Die Versuchspflanzen wurden regelmäßig mit demineralisiertem Wasser bewässert. Ja nach Sonneneinstrahlung und den daraus folgenden Temperaturen erhielten die Pflanzen Wasser nach Bedarf.

\subsection{Schwermetallgaben}

Einen Monat nach dem Umpflanzen in die $2 \mathrm{~kg}$ Versuchstöpfe wurden die SchwermetallGaben in flüssiger Form (50 ml/Topf) direkt in den Wurzelraum der Pflanzen appliziert (Tab.5). Dazu wurden mit einem Pflanzstäbchen 4 Löcher bis zum untersten Drittel der Versuchstöpfe gebohrt und die $50 \mathrm{ml}$ gleichmäßig in die Löcher verteilt. Anschließend wurden die Töpfe gegossen. Die kurz darauf erfolgte Durchwurzelung der Töpfe trug zu noch stärkerer Homogenisierung der Schwermetalle in den Töpfen bei. 
Tab. 5: Zugabestufen an Schwermetallen (mg Element/kg Bodenmischung) und entsprechende Salzmengen (mg Salz/kg Bodenmischung).

Salz

\begin{tabular}{|c|c|c|c|c|c|c|c|}
\hline $\mathrm{ZnSO}_{4} \cdot 7 \mathrm{H}_{2} \mathrm{O}$ & $\begin{array}{l}\mathrm{mg} \mathrm{Zn} / \mathrm{kg} \\
\mathrm{mg} \mathrm{Salz} / \mathrm{kg}\end{array}$ & $\begin{array}{l}0,5 \\
2,20\end{array}$ & $\begin{array}{l}2,5 \\
10,99\end{array}$ & $\begin{array}{l}10 \\
43,98\end{array}$ & $\begin{array}{l}20 \\
87,96\end{array}$ & $\begin{array}{l}40 \\
175,92\end{array}$ & $\begin{array}{l}80 \\
351,84\end{array}$ \\
\hline $\mathrm{CdSO}_{4} \cdot 8 \mathrm{H}_{2} \mathrm{O}$ & $\begin{array}{l}\mathrm{mg} \mathrm{Cd} / \mathrm{kg} \\
\mathrm{mg} \mathrm{Salz} / \mathrm{kg} \\
\end{array}$ & $\begin{array}{l}1,25 \\
8,56 \\
\end{array}$ & $\begin{array}{l}2,5 \\
17,11 \\
\end{array}$ & $\begin{array}{l}5 \\
34,22 \\
\end{array}$ & $\begin{array}{l}10 \\
68,45 \\
\end{array}$ & \begin{tabular}{|l}
20 \\
136,89 \\
\end{tabular} & \\
\hline $\mathrm{Pb}\left(\mathrm{NO}_{3}\right)_{2}$ & $\begin{array}{l}\mathrm{mg} \mathrm{Pb} / \mathrm{kg} \\
\mathrm{mg} \mathrm{N} / \mathrm{kg} \\
\mathrm{mg} \mathrm{Salz/kg}\end{array}$ & $\begin{array}{l}2,5 \\
0,34 \\
3,99\end{array}$ & $\begin{array}{l}5 \\
0,68 \\
7,99\end{array}$ & $\begin{array}{l}10 \\
1,36 \\
15,99\end{array}$ & $\begin{array}{l}20 \\
2,71 \\
31,96\end{array}$ & $\begin{array}{l}40 \\
5,41 \\
63,92\end{array}$ & $\begin{array}{l}80 \\
127,84\end{array}$ \\
\hline
\end{tabular}

Im Versuch 2 wurde der Stickstoff des $\mathrm{Pb}\left(\mathrm{NO}_{3}\right)_{2}$ (Tab.5) von der N-Menge der verwendeten Nährlösung (Tab.4) abgezogen. Die höchste Konzentration ( $80 \mathrm{mg} / \mathrm{kg}$ Boden) von $\mathrm{Zn}$ oder $\mathrm{Pb}$ wurde nur in Versuch 3 verwendet. Die verwendeten Schwermetall-Konzentrationen in allen Versuchen wurden nach institutseigenen Voruntersuchungen und nach Angaben von Fabig (1982), Goergen (1987) und Arduini et al. (1998) ausgewählt.

\subsection{Vermehrung und Beimpfung mit Rhizobien und Frankia}

In Versuch 3.1 mit (A. saligna) wurde eine Mischung aus zwei Rhizobien Arten (Tab. 6a) nach De Laujudie et al. (1994) und Young (1996) verwendet, die von der Deutschen Sammlung für Mikroorganismen und Zellkulturen (DSMZ) stammten. In Versuch 3.2 (mit C. equisetifolia) wurden zwei Frankia-Stämme (Tab.6b), die aus der Sammlung der Naturwissenschaftlichen Fakultät der Suez-Kanal-Universität/Ägypten stammten, zusammengemischt. Die Beimpfung mit Rhizobien oder Frankia erfolgte zwei Wochen nach dem Umpflanzen der vorher beschriebenen Sämlinge in die $2 \mathrm{~kg}$ Versuchstöpfe, wobei $20 \mathrm{ml}$ der Bakterien-Suspension bzw. -Kulturen der jeweiligen Mischung in 4 Löcher im Wurzelraum der Sämlinge appliziert wurden. Die Applikation erfolgte ähnlich wie die SchwermetallApplikation. Die unbeimpften Pflanzen haben dieselbe Menge der Nährlösung (autoklaviert zur Abtötung der Rhizobien oder Frankia) erhalten. 
Tab. 6a: Verwendete Rhizobien-Stämme

\begin{tabular}{|l|l|l|}
\hline Familie & Art & Stamm \\
\hline Rhizobiaceae & Rhizobium $s p$ & DSM 11272 \\
\hline Rhizobiaceae & Sinorhizobium teranga & DSM 11282 \\
\hline
\end{tabular}

Tab. 6b: verwendete Frankia-Stämme

\begin{tabular}{|l|l|}
\hline Familie & Stamm \\
\hline Frankiaceae & I 556 \\
\hline Frankiaceae & I 356 \\
\hline
\end{tabular}

Die Rhizobien wurden im Medium 98 (Tab. 7a, DSM 1983) und die Frankia im Medium 552 (Tab. 7b, DSM 1993) vermehrt. Die Vermehrung erfolgte in einer autoklavierten Nährlösung, deren chemische Zusammensetzung in Tabelle 7a (für Rhizobien) und in Tabelle 7 b (für Frankia) aufgeführt ist. Die pH-Werte wurden bei 7 eingestellt.

Tab. 7a: DSM-Medium 98

\begin{tabular}{|l|l|l|}
\hline Hefe & $1,0 \mathrm{~g}$ & $\mathrm{pH}=7,0$ \\
Mannit & $10,0 \mathrm{~g}$ & \\
Bodenextrakt* & $200,0 \mathrm{ml}$ & \\
Aqua dest. & $800,0 \mathrm{ml}$ & \\
& $80,0 \mathrm{~g}$ & unbehandelter Gartenboden \\
*Bodenextrakt: & $0,2 \mathrm{~g}$ & $\mathrm{Na}_{2} \mathrm{CO}_{3}$ \\
& $200,0 \mathrm{ml}$ & $\mathrm{Aqua}_{\text {dest. }}$ \\
\hline
\end{tabular}

* Der verwendete Bodenextrakt wurde aus $80 \mathrm{~g}$ chemisch unbehandeltem Gartenboden, 0,2 g $\mathrm{Na}_{2} \mathrm{CO}_{3}$ und $200 \mathrm{ml}$ Aqua dest. hergestellt und anschließend filtriert. Die Nährlösung wurde mit dem Rhizobienstamm beimpft und auf dem Schüttelgerät 3 Tage unter Zimmertemperatur geschüttelt. 
Tab. 7b: DSM-Medium 552

\begin{tabular}{|c|c|c|}
\hline $\begin{array}{l}\text { Glucose } \\
\text { Stärke, löslich } \\
\text { Hefe } \\
\mathrm{N}-\mathrm{Z} \text {-Amine } \\
\mathrm{CaCO}_{3} \\
\text { Demi -Wasser }\end{array}$ & $\begin{array}{ll}10,0 & \mathrm{~g} \\
20,0 & \mathrm{~g} \\
5,0 & \mathrm{~g} \\
5,0 & \mathrm{~g} \\
1,0 & \mathrm{~g} \\
1000,0 & \mathrm{ml}\end{array}$ & $\mathrm{pH}=7,0$ \\
\hline
\end{tabular}

\subsection{Versuchauswertung}

\subsubsection{Ernte}

10 Wochen nach der Schwermetall-Behandlung wurden die oberirdischen Pflanzenteile unmittelbar über der Bodenoberfläche abgeschnitten, in Tüten abgepackt und im Trockenschrank bei $80^{\circ} \mathrm{C}$ bis zur Gewichtskonstanz getrocknet und anschließend die Sprosstrockengewichte ermittelt. Die Wurzeln wurden in einer Waschanlage von anhaftendem Bodensubstrat gereinigt, ebenfalls bis zur Gewichtskonstanz getrocknet und gewogen.

\subsubsection{Mineralstoffanalyse}

Die getrockneten Spross-und Wurzelteile wurden gemahlen, und anschließend im Spross die Elemente $\mathrm{P}, \mathrm{K}, \mathrm{Ca}, \mathrm{Mg}, \mathrm{Zn}, \mathrm{Cd}$ und $\mathrm{Pb}$ und in den Wurzeln nur die Schwermetalle $\mathrm{Zn}, \mathrm{Cd}$ und $\mathrm{Pb}$ bestimmt. Die P-Konzentration im aufgeschlossenen Pflanzenmaterial $(<1 \mathrm{~mm})$ wurde koloritmetrisch nach der Vanadat-Molybdat-Methode (KITSON und Mellon, 1944 wie sollen die Namen denn geschrieben werden) bei einer Wellenlänge von $420 \mathrm{~nm}$ mit einem „Technicon Autoanalyser II“ ermittelt. Die Bestimmung der Elemente K, Mg und Ca sowie der Schwermetalle $\mathrm{Zn}, \mathrm{Cd}$ und $\mathrm{Pb}$ erfolgte mit dem Atomabsorptions-Spektrophotometer (AAS, PU9200X/Philips). Die Analyse des Stickstoffs wurde durch die Elementaranalyse mit CARLO-ERBA Series 2, NA 1500 durchgeführt.

\subsubsection{Boden-Analyse}

Der pH-Wert des Bodens wurde am Ende jedes Versuches in 0,01 $\mathrm{M} \mathrm{CaCl}_{2}$-Lösung gemessen.

\subsubsection{Statistische Auswertung}

In allen Versuchen wurde, jede Behandlung viermal wiederholt. Bei allen Versuchen wurden die Ergebnisse mit dem Programm „SYSTAT for Windows, Version 5“ SYSTAT Inc. statistisch analysiert. Die Berechnung der Grenzdifferenzen für Mehrfachvergleiche erfolgte mit dem FISHER-Test $P$ 5\% 


\section{Ergebnisse}

Die vorliegende Arbeit umfasst drei Gefäßversuche, die unter Gewächshausbedingungen durchgeführt worden sind.

\subsection{Versuch 1}

In diesem Versuch wurde der Einfluss der Konzentrationssteigerungen der Schwermetalle Zn, $\mathrm{Cd}$ und $\mathrm{Pb}$ auf das Wachstum und die Konzentration bzw. die Aufnahme von $\mathrm{N}, \mathrm{P}, \mathrm{K}, \mathrm{Ca}$, $\mathrm{Mg}, \mathrm{Zn}, \mathrm{Cd}$ und $\mathrm{Pb}$ im Spross, und von den Schwermetallen $\mathrm{Zn}, \mathrm{Cd}$ und $\mathrm{Pb}$ in den Wurzeln von Pflanzen bei normaler Düngung untersucht.

\subsubsection{Acacia saligna}

\subsubsection{Zn-Behandlung}

\subsection{Sprosstrockengewicht (STG)}

Das Sprosstrockengewicht nahm mit steigender Zn-Konzentration im Boden allgemein geringfügig zu, mit Ausnahme der Konzentration $10 \mathrm{mg} / \mathrm{kg}$ Boden, wo das STG mehr als doppelt so hoch wie in der Kontrolle lag (Tab. 8). Die höchste Zn-Behandlung erhöhte der STG um $20 \%$ verglichen mit der Kontrolle.

Tab.8: Einfluss der Zn-Behandlung (mg/kg Boden) auf das Sprosstrockengewicht (g/Topf) von Acacia saligna

\begin{tabular}{|l|l|l|l|l|l|l|l|}
\hline mg Zn/kg Boden & 0,0 & 0,5 & 2,5 & 10 & 20 & 40 & GD 5\% \\
\hline STG g/Topf & 2,66 & 3,62 & 3,47 & 5,51 & 3,97 & 3,18 & 1,68 \\
\hline
\end{tabular}

\subsection{Wurzeltrockengewicht (WTG)}

Die TS-Bildung in den Wurzeln zeigte einen ähnlichen Verlauf wie im Spross, jedoch auf niedrigerem Niveau (Tab.9). Dies bedeutet, dass das WTG mit steigender Zn-Zufuhr anstieg, wobei der Unterschied nur bei der Behandlung $10 \mathrm{mg} / \mathrm{kg}$ Boden signifikant gegenüber der Kontrolle war. Diese Behandlung bewirkte eine 2,6fache Erhöhung der WTG. Anders als beim Spross, erhöhte sich das WTG stärker bei der höchsten Zn-Stufe, und zwar um etwa $45 \%$ gegenüber der Kontrolle. 
Tab.9: Einfluss der Zn-Behandlung (mg/kg Boden) auf das Wurzeltrockengewicht (g/Topf) von Acacia saligna

\begin{tabular}{|l|l|l|l|l|l|l|l|}
\hline mg Zn/kg Boden & 0,0 & 0,5 & 2,5 & 10 & 20 & 40 & GD 5 \% \\
\hline WTG g/Topf & 0,71 & 1,20 & 1,17 & 1,84 & 1,21 & 1,03 & 0,93 \\
\hline
\end{tabular}

5.1.1.1.3 Konzentration und Aufnahme von N, P, K, Ca, Mg und Zn und im Spross

\section{$\mathrm{N}$-Konzentration und -Aufnahme}

Bis zur Zugabe von $20 \mathrm{mg} \mathrm{Zn/kg}$ veränderten sich die N-Konzentrationen in der Sprosstrockensubstanz von A. saligna nicht signifikant, erst bei $40 \mathrm{mg} / \mathrm{kg}$ nahm die NKonzentration um $1 / 3$ gegenüber der Kontrolle ab (Tab.10). Die N-Aufnahme stieg mit steigender Zn-Zufuhr zwar an, aber diese Steigerung war, mit Ausnahme der Zn-Stufe $10 \mathrm{mg} / \mathrm{kg}$ Boden, weder signifikant gegenüber der Kontrolle noch proportional zum Wachstum. Zum Beispiel erhöhte sich die N-Aufnahme im Spross bei der Zn-Stufe 10 mg/kg Boden um $69 \%$. Der STG wurde hier aber verdoppelt.

Tab.10: Einfluss der Zn-Behandlung (mg/kg Boden) auf die Konzentration (mg/g TS) und Aufnahme (mg/Topf) von N im Spross von Acacia saligna

\begin{tabular}{|l|r|r|}
\hline mg Zn/kg Boden & $\begin{array}{l}\text { N-Konzentration } \\
\text { (mg/g TS) }\end{array}$ & $\begin{array}{l}\text { N-Aufnahme } \\
\text { (mg/Topf) }\end{array}$ \\
\hline 0,0 & 23,8 & 60,3 \\
0,5 & 17,5 & 61,6 \\
2,5 & 19,1 & 66,2 \\
10 & 18,7 & 101,9 \\
20 & 20,1 & 86,7 \\
40 & 15,8 & 49,1 \\
\hline GD 5\% & 6,5 & 38,6 \\
\hline
\end{tabular}




\section{P-Konzentration und -Aufnahme}

Mit steigender Zn-Konzentration im Boden nahm die P-Konzentration im Spross ab, aber erst bei den Zn-Konzentrationen 10, 20 und 40 mg/kg Boden signifikant zur Kontrolle (Tab.11). Die P-Konzentration bei der höchsten Zn-Stufe lag aber höher als die der vorherigen Stufe $20 \mathrm{mg} \mathrm{Zn} / \mathrm{kg}$ Boden. Die P-Aufnahme entsprach dem STG, wobei die höchste P-Aufnahme beim höchsten STG und der Zn-Stufe $10 \mathrm{mg} / \mathrm{kg}$ Boden festgestellt wurde. Wegen des hohen GD-Wertes waren die P-Aufnahmen aller Zn-Behandlungen nicht signifikant verschieden zur Kontrolle.

Tab.11: Einfluss der Zn-Behandlung (mg/kg Boden) auf die Konzentration (mg/g TS) und Aufnahme (mg/Topf) von P, K, Ca und Mg im Spross von Acacia saligna

\begin{tabular}{|l|llll|lllll|}
\hline \multirow{2}{*}{ mg Zn/kg Boden } & \multicolumn{7}{|l|}{ Konzentration (mg/gTS) } & \multicolumn{4}{|l}{ Aufnahme (mg/Topf) } \\
\cline { 2 - 9 } & $\mathrm{P}$ & $\mathrm{K}$ & $\mathrm{Ca}$ & $\mathrm{Mg}$ & $\mathrm{P}$ & $\mathrm{K}$ & $\mathrm{Ca}$ & $\mathrm{Mg}$ \\
\hline \multirow{2}{*}{0,0} & 4,6 & 15,5 & 16,1 & 3,3 & 11,9 & 40,0 & 42,7 & 8,7 \\
0,5 & 4,3 & 13,3 & 15,8 & 2,8 & 15,5 & 47,2 & 56,3 & 10,0 \\
2,5 & 4,0 & 12 & 15,9 & 3,7 & 13,8 & 41,4 & 54,5 & 12,9 \\
10 & 3,0 & 8,5 & 13,0 & 2,3 & 16,5 & 47,0 & 71,0 & 12,5 \\
20 & 2,35 & 8,8 & 10,6 & 2,2 & 9,3 & 34,5 & 47,1 & 9,9 \\
40 & 3,3 & 9,8 & 12,2 & 2,5 & 10,4 & 30,3 & 38,1 & 7,9 \\
\hline \multirow{2}{*}{ GD 5\% } & 1,1 & 1,7 & 4,8 & 0,8 & $\mathrm{~ns}$ & $\mathrm{~ns}$ & $\mathrm{~ns}$ & $\mathrm{~ns}$ \\
\hline
\end{tabular}

\section{K-Konzentration und -Aufnahme}

Wie bei den vorher besprochenen Elementen wurde auch beim $\mathrm{K}$ ein abnehmender Trend der Konzentration mit zunehmender Zn-Zufuhr zum Boden ermittelt (Tab.11). Auffallend war die starke Abnahme um $45 \%$ gegenüber der Kontrolle bei der Behandlung $10 \mathrm{mg} \mathrm{Zn/kg} \mathrm{Boden.}$ $\mathrm{Ab}$ dieser $\mathrm{Zn}$-Stufe lag die K-Konzentration signifikant niedriger als in der Kontrolle. Die KAufnahmen wiesen auch bei der Zn-Zugabe von $10 \mathrm{mg} / \mathrm{kg}$ Boden, wo das höchste STG, aber die niedrigste K-Konzentration festgestellt wurde, keine signifikanten Unterschiede gegenüber der Kontrolle auf. 


\section{Ca-Konzentration und -Aufnahme}

Mit Ausnahme der niedrigen Ca-Konzentration der Zn-Stufe 20 mg/kg Boden (34 \% unter der Kontrolle) waren die Konzentrationen dieses Elementes weder gegenüber der Kontrolle noch untereinander signifikant verschieden (Tab.11). Entsprechend dem Wachstum nahm die CaAufnahme bis zur Zn-Stufe $10 \mathrm{mg} / \mathrm{kg}$ Boden zu, wobei die bei dieser Stufe am stärksten gewachsenen Pflanzen auch mit um 66 \% über der Kontrolle die höchste Ca-Aufnahme aufwiesen. Nach dieser Zn-Stufe nahm die Ca-Aufnahme wieder ab.

\section{Mg-Konzentration und-Aufnahme}

Die Mg-Konzentration nahm ab der Zn-Stufe $10 \mathrm{mg} / \mathrm{kg}$ Boden deutlich ab (Tab.11). Die MgAufnahme zeigte einen ähnlichen Verlauf wie die TS-Bildung. Auffallend war jedoch, dass die Pflanzen, welche das höchste STG bei der Zn-Stufe $10 \mathrm{mg} / \mathrm{kg}$ Boden erbrachten, nicht die höchste Mg-Aufnahme aufwiesen. Daraus resultieren die sehr niedrigen Mg-Konzentrationen bei 10 und $20 \mathrm{mg} \mathrm{Zn/kg}$.

\section{Zn-Konzentration und-Aufnahme}

Mit Ausnahme der niedrigsten Zn-Stufe (0,5 mg/kg Boden), wo nur eine geringe Erhöhung der Zn-Konzentration im Acacia-Spross festzustellen war, wurden die Zn-Konzentrationen mit steigender Zn-Zufuhr zum Boden bei 2,5; 10; 20 und $40 \mathrm{mg} / \mathrm{kg}$ Boden signifikant gegenüber der Kontrolle auf das 3; 3,8; 6,8 und 8fache erhöht (Tab.12). Die Zn-Aufnahme stieg ebenfalls an, und erreichte das 9,7fache bei der höchsten Zn-Zugabe verglichen mit der Kontrolle. Im allgemeinen verlief sowohl die Zn-Konzentration als auch die Zn-Aufnahme deutlich proportional zur steigenden Zn-Zufuhr und nicht zum Wachstum. 
Tab.12: Einfluss der Zn-Behandlung (mg/kg Boden) auf die Konzentration (mg/g TS) und Aufnahme (mg/Topf) von Zn im Spross von Acacia saligna

\begin{tabular}{|l|r|r|}
\hline mg Zn/kg Boden & $\begin{array}{l}\text { Zn-Konzentration } \\
\text { (mg/g TS) }\end{array}$ & $\begin{array}{l}\text { Zn-Aufnahme } \\
\text { (mg/Topf) }\end{array}$ \\
\hline 0,0 & 0,05 & 0,13 \\
0,5 & 0,06 & 0,23 \\
2,5 & 0,15 & 0,52 \\
10 & 0,19 & 1,03 \\
20 & 0,34 & 1,27 \\
40 & 0,40 & 1,26 \\
\hline \multirow{2}{*}{ GD 5\% } & 0,07 & 0,58 \\
\hline
\end{tabular}

\subsection{Zn-Konzentration und -Aufnahme in der Wurzel}

Die Werte beider Parameter nahmen mit seigender Zn-Konzentration im Boden zu (Tab.13). Diese Steigerung war nur bei den beiden höchsten Zn-Stufen für die Zn-Konzentration (auf das 7,3 und 17,1fache) und ab der Zn-Stufe $10 \mathrm{mg} / \mathrm{kg}$ Boden für die Zn-Aufnahme (auf das 25,4fache bei der höchsten Zn-Zugabe) signifikant im Vergleich zur Kontrolle. 
Tab. 13: Einfluss der Zn-Behandlung (mg/kg Boden) auf die Konzentration (mg/g TS) und Aufnahme (mg/Topf) von $\mathrm{Zn}$ in der Wurzel von Acacia saligna

\begin{tabular}{|l|r|r|}
\hline mg Zn/kg Boden & $\begin{array}{l}\text { Zn-Konzentration } \\
\text { (mg/g TS) }\end{array}$ & $\begin{array}{l}\text { Zn-Aufnahme } \\
\text { (mg/Topf) }\end{array}$ \\
\hline 0,0 & 0,12 & 0,08 \\
0,5 & 0,20 & 0,24 \\
2,5 & 0,41 & 0,46 \\
10 & 0,53 & 0,97 \\
20 & 0,88 & 1,07 \\
40 & 2,05 & 2,03 \\
\hline GD 5\% & 0,45 & 0,87 \\
\hline
\end{tabular}

\subsection{Der pH-Wert des Bodens}

In allen Varianten sank das Ausgangsboden- $\mathrm{pH}$ von 7,0 bis auf 4,6 am Ende der Vegetationsperiode $\mathrm{ab}$ (Tab.14). Die Unterschiede zwischen den $\mathrm{pH}$-Werten der verschiedenen Zn-Behandlungen waren nicht signifikant im Vergleich zur Kontrolle .

Tab.14: Einfluss der Zn-Behandlung (mg/kg Boden) auf den pH-Wert im Boden von Acacia saligna am Ende des Versuches

\begin{tabular}{|l|l|l|l|l|l|l|l|}
\hline $\mathrm{mg} \mathrm{Zn} / \mathrm{kg}$ Boden & 0,0 & 0,5 & 2,5 & 10 & 20 & 40 & GD 5\% \\
\hline $\mathrm{pH}$ & 4,8 & 4,7 & 4,7 & 4,8 & 4,9 & 4,6 & $\mathrm{~ns}$ \\
\hline
\end{tabular}




\subsubsection{Cd-Behandlung}

\subsection{Sprosstrockengewicht (STG)}

Die Behandlung des Bodens der Acacia-Sämlinge mit Cd erhöhte die TS-Bildung im Spross um $48 \%$ bei der Cd -Stufe 2,5 ppm und um $60 \%$ bei 5 ppm Cd signifikant gegenüber der Kontrolle (Tab.15). Die weitere Steigerung der Cd-Zufuhr führte zu einer starken Abnahme der STG, die bei der höchsten Cd-Zugabe von $20 \mathrm{mg} / \mathrm{kg}$ Boden nur noch $51 \%$ der Kontrolle erreichten.

Tab.15: Einfluss der Cd-Behandlung (mg/kg Boden) auf das Sprosstrockengewicht (g/Topf) von Acacia saligna

\begin{tabular}{|l|l|l|l|l|l|l|l|}
\hline mg Cd/kg Boden & 0,0 & 1,25 & 2,5 & 5 & 10 & 20 & GD 5 \% \\
\hline STG g/Topf & 2,87 & 3,85 & 4,26 & 4,59 & 2,64 & 1,47 & 1,09 \\
\hline
\end{tabular}

\subsection{Wurzeltrockengewicht (WTG)}

Die TS-Bildung in den Wurzeln verlief ähnlich wie im Spross, in dem sie bis zur Cd-Stufe 5 ppm anstieg (Tab.16). Diese Steigerung war jedoch relativ viel höher als beim Spross, insbesondere bei der Cd-Behandlung $5 \mathrm{mg} / \mathrm{kg}$ Boden, in der das WTG auf das 2,7fache verglichen mit der Kontrolle erhöht wurde, gegenüber nur $60 \%$ beim Spross. Die weitere Erhöhung der Cd-Konzentration im Boden beeinträchtigte das WTG, wobei die Unterschiede wegen starker Streuung der Werte und dem entsprechend hohen GD-Wertes nicht signifikant gegenüber der Kontrolle waren.

Tab.16:Einfluss der Cd-Behandlung (mg/kg Boden) auf das Wurzeltrockengewicht (g/Topf) von Acacia saligna

\begin{tabular}{|l|l|l|l|l|l|l|l|}
\hline Cd mg/kg Boden & 0,0 & 1,25 & 2,5 & 5 & 10 & 20 & GD 5\% \\
\hline WTG g/Topf & 0,7 & 1,2 & 1,38 & 1,86 & 0,55 & 0,19 & 0,75 \\
\hline
\end{tabular}




\subsection{Konzentration und Aufnahme von N, P, K, Ca, Mg und Cd im}

Spross

\section{N-Konzentration und -Aufnahme}

Mit steigender Cd-Zufuhr bis 5 ppm sank die N-Konzentration im Spross der AcaciaPflanzen nicht signifikant zur Kontrolle ab (Tab.17). Bei der höchsten Cd-Zugabe, wo das Wachstum der Pflanzen am stärksten beeinträchtigt wurde, akkumulierte der Spross signifikant mehr $\mathrm{N}$ als in der Kontrolle und den Behandlungen bis $5 \mathrm{mg} / \mathrm{kg}$ Boden. Die $\mathrm{N}$ Aufnahme verlief mehr oder weniger ähnlich wie die TS-Bildung.

Tab.17: Einfluss der Cd-Behandlung (mg/kg Boden) auf die Konzentration (mg/g TS) und Aufnahme (mg/Topf) von N im Spross von Acacia saligna

\begin{tabular}{|l|r|r|}
\hline mg Cd/kg Boden & $\begin{array}{l}\text { N-Konzentration } \\
\text { (mg/g TS })\end{array}$ & $\begin{array}{l}\text { N-Aufnahme } \\
\text { (mg/Topf })\end{array}$ \\
\hline 0,0 & 21,2 & 60,3 \\
1,25 & 18,0 & 69,2 \\
2,5 & 16,7 & 71,4 \\
5 & 18,4 & 84,9 \\
10 & 22,1 & 56,4 \\
20 & 26,0 & 36,1 \\
\hline GD $5 \%$ & 4,8 & 23,5 \\
\hline
\end{tabular}

\section{P-Konzentration und -Aufnahme}

Obwohl die P-Konzentration in der Sprossmasse bei Erhöhung der Cd-Konzentration im Boden abnahm, waren ihre Werte jedoch nicht signifikant niedriger als in der Kontrolle (Tab.18). Die P-Aufnahme zeigte eine ähnliche Tendenz wie die TS-Bildung. Auffallend war die stärker reduzierte P-Aufnahme bei der höchsten Cd-Zugabe im Vergleich zur Abnahme des STG. 
Tab.18: Einfluss der Cd-Behandlung (mg/kg Boden) auf die Konzentration (mg/g TS) und Aufnahme (mg/Topf) von P, K, Ca und Mg im Spross von Acacia saligna

\begin{tabular}{|l|llll|lllll|}
\hline \multirow{2}{*}{$\mathrm{mg} \mathrm{Cd} / \mathrm{kg}$ Boden } & \multicolumn{4}{|l|}{ Konzentration (mg/gTS) } & \multicolumn{4}{|c|}{ Aufnahme (mg/Topf) } \\
\cline { 2 - 9 } & $\mathrm{P}$ & $\mathrm{K}$ & $\mathrm{Ca}$ & $\mathrm{Mg}$ & $\mathrm{P}$ & $\mathrm{K}$ & $\mathrm{Ca}$ & $\mathrm{Mg}$ \\
\hline 0,0 & 4,5 & 14,8 & 13,3 & 3,2 & 13,0 & 41,9 & 38,2 & 9,3 \\
1,25 & 4,3 & 13,7 & 13,2 & 3,5 & 16,5 & 52,8 & 50,5 & 13,4 \\
2,5 & 4,1 & 11,7 & 10,9 & 2,9 & 16,9 & 49,1 & 46,4 & 12,2 \\
5 & 4,1 & 12,3 & 12,1 & 2,7 & 18,8 & 57,1 & 55,5 & 12,2 \\
10 & 3,2 & 13,4 & 11,3 & 2,5 & 8,4 & 35,5 & 28,9 & 6,4 \\
20 & 3,2 & 15,5 & 12,4 & 2,4 & 5,2 & 22,8 & 17,5 & 3,6 \\
\hline \multirow{2}{*}{ GD 5\% } & $\mathrm{ns}$ & $\mathrm{ns}$ & $\mathrm{ns}$ & 0,7 & 6,4 & 16,4 & 12,4 & 3,2 \\
\hline
\end{tabular}

K-Konzentration und -Aufnahme

Die K-Konzentration zeigte zwar eine schwache Abnahme bzw. Verdünnung im Spross der am stärksten gewachsenen Pflanzen (bis 2,5 und 5 ppm Cd-Zufuhr), aber die Unterschiede waren weder zwischen den Cd-Stufen noch gegenüber der Kontrolle signifikant (Tab.18). Wie bei N und P verlief die K-Aufnahme ähnlich wie beim STG.

Ca-Konzentration und -Aufnahme

Die Acacia-Pflanzen akkumulierten das $\mathrm{Ca}$ im Spross auf einem ähnlichen Niveau wie $\mathrm{K}$ (Tab.18). Die Ca-Aufnahme zeigte einen ähnlichen Trend wie die TS-Bildung.

Mg-Konzentration und -Aufnahme

Obwohl die Mg-Konzentrationen und -Aufnahmen mengenmäßig deutlich niedriger lagen als bei $\mathrm{K}$ und $\mathrm{Ca}$, wiesen beide Parameter bei den drei Nährstoffen ähnliche Tendenzen auf (Tab.18).

Cd-Konzentration und -Aufnahme

Die Cd-Konzentration stieg im Spross der Acacia-Pflanzen mit zunehmender CdAnwendung zum Boden bis zur höchsten Cd-Stufe drastisch an (Tab.19). Bei höheren Cd Zugaben nahm die Cd-Konzentration im Spross zwar zu, jedoch war diese Zunahme nicht 
proportional zu den steigenden Cd-Stufen. Die Zugabe von 10 und 20 ppm Cd zum Boden führte zu einer drastischen Erhöhung der Cd-Konzentration im Spross gegenüber der Kontrolle. Die höchste Cd-Aufnahme wurde entsprechend dem Wachstum bei 5 ppm Cd festgestellt.

Tab.19: Einfluss der Cd-Behandlung (mg/kg Boden) auf die Konzentration (mg/g TS) und Aufnahme (mg/Topf) von Cd im Spross von Acacia saligna

\begin{tabular}{|l|r|r|}
\hline mg Cd/kg Boden & $\begin{array}{l}\text { Cd-Konzentration } \\
\text { (mg/g TS })\end{array}$ & $\begin{array}{l}\text { Cd-Aufnahme } \\
\text { (mg/Topf) }\end{array}$ \\
\hline 0,0 & 0,00 & 0,00 \\
1,25 & 0,03 & 0,13 \\
2,5 & 0,05 & 0,20 \\
5 & 0,08 & 0,37 \\
10 & 0,11 & 0,29 \\
20 & 0,11 & 0,16 \\
\hline GD 5\% & 0,02 & 0,10 \\
\hline
\end{tabular}

\subsection{Cd-Konzentration und -Aufnahme in der Wurzel}

Im Vergleich zum Spross wies die Wurzel viel höhere Cd-Konzentrationen auf, insbesondere bei beiden höchsten Cd-Stufen (Tab.20). Ebenfalls lag die Cd-Aufnahme in der Wurzel im allgemeinen höher als im Spross, wobei die höchste Aufnahme in den Wurzeln der am stärksten gewachsenen Pflanzen ermittelt werden konnten. Auffallend war die ausgeprägte Giftigkeit der höchsten Cd-Zugabe auf die TS-Bildung der Wurzeln. 
Tab.20: Einfluss der Cd-Behandlung ( $\mathrm{mg} / \mathrm{kg}$ Boden) auf die Konzentration (mg/g TS) und Aufnahme (mg/Topf) von Cd in der Wurzel von Acacia saligna

\begin{tabular}{|l|r|r|}
\hline $\mathrm{mg} \mathrm{Cd} / \mathrm{kg}$ Boden & $\begin{array}{l}\text { Cd-Konzentration } \\
\text { (mg/g TS) }\end{array}$ & $\begin{array}{l}\text { Cd-Aufnahme } \\
\text { (mg/Topf) }\end{array}$ \\
\hline 0,0 & 0,00 & 0,00 \\
1,25 & 0,27 & 0,29 \\
2,5 & 0,36 & 0,48 \\
5 & 0,43 & 0,77 \\
10 & 0,77 & 0,31 \\
20 & 1,36 & 0,25 \\
\hline GD 5\% & 0,30 & 0,26 \\
\hline
\end{tabular}

\subsection{Der $\mathrm{pH}-$ Wert des Bodens}

Wie bei der Zn-Zufuhr zum Boden, sank das Ausgangs-pH des Boden von 7 auf Werte bis 4,8 am Ende der Vegetationsperiode ab (Tab.21).

Tab. 21: Einfluss der Cd-Behandlung (mg/kg Boden) auf den pH-Wert im Boden von Acacia saligna am Ende des Versuches

\begin{tabular}{|l|r|r|r|r|r|r|c|}
\hline $\mathrm{mg} \mathrm{Cd} / \mathrm{kg}$ Boden & 0,0 & 1,25 & 2,5 & 5 & 10 & 20 & GD 5\% \\
\hline $\mathrm{pH}$ & 4,8 & 4,8 & 4,9 & 4,9 & 5,2 & 5,4 & 0,3 \\
\hline
\end{tabular}




\subsubsection{Pb-Behandlung}

\subsection{Sprosstrockengewicht (STG)}

Mit zunehmender Pb-Gabe zum Boden bis 20 ppm stiegen die Sprosstrockengewichte der Pflanzen leicht an (Tab.22). Der höchste Wert wurde bei einer Pb-Konzentration von $10 \mathrm{mg} / \mathrm{kg}$ Boden festgestellt. Eine Erhöhung der Pb-Zugabe auf $40 \mathrm{mg} / \mathrm{kg}$ Boden führte zu einer Abnahme des Sprosstrockengewichtes. Signifikante Unterschiede zwischen den Behandlungen und der Kontrolle konnten nicht festgestellt werden.

Tab.22: Einfluss der Pb-Behandlung (mg/kg Boden) auf das Sprosstrockengewicht (g/Topf) von Acacia saligna

\begin{tabular}{|l|c|c|c|c|c|c|l|}
\hline mg Pb/kg Boden & 0,0 & 2,5 & 5 & 10 & 20 & 40 & GD 5\% \\
\hline STG g/Topf & 3,33 & 3,46 & 3,61 & 3,81 & 3,70 & 2,73 & ns \\
\hline
\end{tabular}

\subsection{Wurzeltrockengewicht (WTG)}

Das Wurzeltrockengewicht von A. saligna nahm mit steigenden $\mathrm{Pb}$-Konzentrationen im Boden zu (Tab.23). Der maximale Wert wurde bei einer Zugabe von $20 \mathrm{mg} / \mathrm{kg}$ Boden festgestellt. Das Wurzeltrockengewicht der behandelten Pflanzen war bei den Stufen 5, 10 und $20 \mathrm{mg} / \mathrm{kg}$ Boden signifikant höher als das der Kontrollpflanzen. Das WTG der Pflanzen nahm bei der höchsten Pb-Stufe (40 mg/kg Boden) wieder ab, lag jedoch noch etwas höher als bei den Kontrollpflanzen.

Tab.23: Einfluss der Pb-Behandlung (mg/kg Boden) auf das Wurzeltrockengewicht (g/Topf) von Acacia saligna

\begin{tabular}{|l|r|r|r|r|r|r|l|}
\hline mg Pb/kg Boden & 0,0 & 2,5 & 5 & 10 & 20 & 40 & GD 5\% \\
\hline WTG g/Topf & 0,77 & 1,08 & 1,14 & 1,22 & 1,23 & 0,89 & 0,34 \\
\hline
\end{tabular}

5.1.1.3.3 Konzentration und Aufnahme von N, P, K, Ca, $\mathrm{Mg}$ und $\mathrm{Pb}$ im Spross

N-Konzentration und -Aufnahme

Obwohl das verabreichte Bleinitrat Stickstoff enthielt, zeigten die N-Konzentrationen der Pflanzen keine signifikanten Unterschiede zwischen den $\mathrm{Pb}$ behandelten Pflanzen und den Kontrollpflanzen (Tab.24). Die N-Aufnahme stieg mit zunehmender Pb-Konzentration an. 
Die signifikant höchste $\mathrm{N}$-Aufnahme wurde beim höchsten STG bei Pb-Zugabe von $10 \mathrm{mg} / \mathrm{kg}$ Boden festgestellt. Die Zufuhr von $40 \mathrm{mg} \mathrm{Pb} / \mathrm{kg}$ zum Boden führte zu einer signifikanten Abnahme der N-Aufnahme gegenüber der Kontrolle.

Tab.24: Einfluss der Pb-Behandlung (mg/kg Boden) auf die Konzentration (mg/g TS) und Aufnahme (mg/Topf) von N im Spross von Acacia saligna

\begin{tabular}{|l|r|r|}
\hline mg Pb/kg Boden & $\begin{array}{l}\text { N-Konzentration } \\
\text { (mg/g TS })\end{array}$ & $\begin{array}{l}\text { N-Aufnahme } \\
\text { (mg/Topf) }\end{array}$ \\
\hline 0,0 & 20,6 & 67,1 \\
2,5 & 19,1 & 65,0 \\
5 & 18,7 & 67,4 \\
10 & 21,1 & 78,8 \\
20 & 19,1 & 69,4 \\
40 & 22,2 & 57,8 \\
\hline GD $5 \%$ & $\mathrm{~ns}$ & 6,9 \\
\hline
\end{tabular}

P-Konzentration und -Aufnahme

Weder die P-Konzentration noch die P-Aufnahme im Spross von A. saligna wurden durch die steigende $\mathrm{Pb}$-Konzentration bis $\mathrm{zu} 40 \mathrm{mg} / \mathrm{kg}$ Boden signifikant verändert (Tab.25). 
Tab.25: Einfluss der Pb-Behandlung ( $\mathrm{mg} / \mathrm{kg}$ Boden) auf die Konzentration (mg/gTS) und Aufnahme (mg/Topf) von P, K, Ca und Mg im Spross von Acacia saligna

\begin{tabular}{|l|llll|lllll|}
\hline \multirow{2}{*}{$\mathrm{mg} \mathrm{Pb} / \mathrm{kg}$ Boden } & \multicolumn{6}{|l|}{ Konzentration (mg/g TS) } & \multicolumn{4}{|c|}{ Aufnahme (mg/Topf) } \\
\cline { 2 - 9 } & $\mathrm{P}$ & $\mathrm{K}$ & $\mathrm{Ca}$ & $\mathrm{Mg}$ & $\mathrm{P}$ & $\mathrm{K}$ & $\mathrm{Ca}$ & $\mathrm{Mg}$ \\
\hline 0,0 & 3,9 & 15,3 & 14,9 & 3,1 & 12,9 & 50,1 & 48,7 & 10,2 \\
2,5 & 4,0 & 14,9 & 15,3 & 3,0 & 13,6 & 50,8 & 52,9 & 10,4 \\
5 & 3,7 & 12,1 & 14,3 & 3,0 & 13,2 & 43,5 & 51,6 & 10,7 \\
10 & 3,2 & 11,5 & 13,9 & 2,8 & 12,0 & 43,5 & 51,9 & 10,6 \\
20 & 3,4 & 12,0 & 16,2 & 1,8 & 12,3 & 44,5 & 60,9 & 6,5 \\
40 & 4,7 & 15,4 & 17,0 & 1,6 & 12,8 & 41,6 & 45,9 & 4,4 \\
\hline \multirow{2}{*}{$\mathrm{GD} 5 \%$} & $\mathrm{~ns}$ & 2,7 & $\mathrm{~ns}$ & 0,9 & $\mathrm{~ns}$ & 8,3 & $\mathrm{~ns}$ & 3,1 \\
\hline
\end{tabular}

K-Konzentration und -Aufnahme

Mit steigender $\mathrm{Pb}$-Konzentration im Boden nahm die K-Konzentration der Pflanzen meistens leicht ab (Tab.25). Signifikante Verringerungen der K-Konzentration gegenüber der Kontrolle wurden bei 5, 10, $20 \mathrm{mg} \mathrm{Pb} / \mathrm{kg}$ Boden festgestellt. Bei einer Konzentration von $10 \mathrm{mg} / \mathrm{kg}$ Boden wiesen die Pflanzen die geringste K-Konzentration auf, die um $25 \%$ unter der Kontrolle lag. Die K-Aufnahme nahm mit zunehmender Pb-Konzentration ab. Der geringste Wert wurde bei der höchsten Pb-Stufe ( $40 \mathrm{mg} / \mathrm{kg}$ Boden) ermittelt. Wegen der deutlichen Abnahme des STG war die K-Konzentration aber ebenso hoch wie in der Kontrolle.

Ca-Konzentration und -Aufnahme

Die Ca-Konzentration im Spross der behandelten Pflanzen unterschied sich nicht signifikant von den Kontrollpflanzen (Tab.25). Bis zu einer Pb-Stufe von 20 ppm stieg die Ca-Aufnahme der Pflanzen an. Signifikante Unterschiede zwischen den Behandlungen wurden nicht festgestellt.

\section{Mg-Konzentration und -Aufnahme}

Die nicht behandelten Kontrollpflanzen zeigten die höchste Mg-Konzentration (Tab.25).

Die Zunahme der Pb-Konzentration im Boden führte erst bei 20 und $40 \mathrm{ppm}$ zu einer 
signifikanten Abnahme der Mg-Konzentration im Spross der Pflanzen um durchschnittlich $45 \%$. Die Mg-Aufnahme unterschied sich nicht zwischen Kontrollpflanzen und Pflanzen, die bei $\mathrm{Pb}$-Gaben von 2,5; 5 oder 10 wuchsen. Bei weiter ansteigenden $\mathrm{Pb}$ Konzentrationen (20 bzw. 40 mg/kg Boden) nahmen die Mg-Aufnahmen der Pflanzen sehr deutlich ab.

\section{$\mathrm{Pb}$-Konzentration und -Aufnahme}

Die $\mathrm{Pb}$-Konzentration im Spross der Pflanzen nahm mit steigender Pb-Zufuhr drastisch zu (Tab.26). Bei einer Pb-Zugabe von $20 \mathrm{mg} \mathrm{Pb/kg}$ Boden erhöhte sich die Pb-Konzentration der Sprosse auf das 13fache. Ebenso stieg die Pb-Aufnahme der Pflanzen mit zunehmender $\mathrm{Pb}$-Konzentration im Boden an. Die Zufuhr von $20 \mathrm{bzw}$. $40 \mathrm{mg} \mathrm{Pb} / \mathrm{kg}$ Boden führte zu einem Anstieg der Pb-Aufnahme auf das 13,2 bzw. 25,5fache der Kontrolle.

Tab.26: Einfluss der Pb-Behandlung (mg/kg Boden) auf die Konzentration (mg/g TS) und Aufnahme (mg/Topf) von Pb im Spross von Acacia saligna

\begin{tabular}{|l|r|r|}
\hline mg Pb/kg Boden & $\begin{array}{c}\text { Pb-Konzentration } \\
(\mathrm{mg} / \mathrm{g} \text { TS })\end{array}$ & $\begin{array}{c}\text { Pb-Aufnahme } \\
\text { (mg/Topf })\end{array}$ \\
\hline 0,0 & 0,004 & 0,014 \\
2,5 & 0,010 & 0,035 \\
5 & 0,017 & 0,062 \\
10 & 0,017 & 0,066 \\
20 & 0,051 & 0,185 \\
40 & 0,132 & 0,357 \\
\hline GD $5 \%$ & 0,015 & 0,067 \\
\hline
\end{tabular}

\subsection{Pb-Konzentration und -Aufnahme in der Wurzel}

Mit zunehmender $\mathrm{Pb}$-Konzentration im Boden akkumulierten die Pflanzen sehr hohe Mengen an $\mathrm{Pb}$ in ihren Wurzeln gegenüber der Kontrolle, so dass die $\mathrm{Pb}$-Aufnahmen der Wurzeln auf viel höherem Niveau als im Spross lagen (Tab.26 und 24). Die PbKonzentrationen und -Aufnahmen der einzelnen Pb-Stufen waren, auch wenn die GDWerte sehr hoch lagen, vielfach höher als in der Kontrolle. 
Tab.27: Einfluss der Pb-Behandlung (mg/kg Boden) auf die Konzentration (mg/g TS) und Aufnahme (mg/Topf) von Pb in der Wurzel von Acacia saligna

\begin{tabular}{|l|r|r|}
\hline mg Pb/kg Boden & $\begin{array}{l}\text { Pb-Konzentration } \\
\text { (mg/g TS) }\end{array}$ & $\begin{array}{l}\text { Pb-Aufnahme } \\
\text { (mg/Topf) }\end{array}$ \\
\hline 0,0 & 0,005 & 0,004 \\
2,5 & 0,087 & 0,089 \\
5 & 0,125 & 0,140 \\
10 & 0,146 & 0,173 \\
20 & 0,318 & 0,402 \\
40 & 0,958 & 0,814 \\
\hline GD 5\% & 0,173 & 0,135 \\
\hline
\end{tabular}

\subsection{Der pH-Wert des Bodens}

Der pH-Wert des Bodens wurde durch die Steigerung der Pb-Konzentrationen im Boden nicht signifikant verändert (Tab.28). Wie bei der Zn-Zufuhr, nahm das Ausgangs-pH des Bodens von 7 auf Werte bis 4,8 am Ende der Vegetationsperiode ab.

Tab.28: Einfluss der Pb-Behandlung (mg/kg Boden) auf den $\mathrm{pH}-W e r t ~ i m$ Boden von Acacia saligna am Ende des Versuches

\begin{tabular}{|l|r|r|r|r|r|r|l|}
\hline $\mathrm{mg} \mathrm{Pb} / \mathrm{kg}$ Boden & 0,0 & 2,5 & 5 & 10 & 20 & 40 & GD 5\% \\
\hline $\mathrm{pH}$ & 4,8 & 4,9 & 4,8 & 4,8 & 4,9 & 4,8 & ns \\
\hline
\end{tabular}




\subsubsection{Casuarina equisetifolia}

\subsubsection{Zn-Behandlung}

\subsection{Sprosstrockengewicht (STG)}

Das Sprosstrockengewicht von C. equisetifolia stieg mit steigender Zn-Konzentration im Boden geringfügig an (Tab.29). Die höchste Zn-Stufe $(40 \mathrm{mg} / \mathrm{kg}$ ) beeinträchtigte das Wachstum der Pflanzen um $20 \%$ gegenüber der Kontrolle. Signifikante Unterschiede im Vergleich zu den nicht behandelten Kontrollpflanzen konnten wegen starker Streuung der Werte nicht festgestellt werden.

Tab.29: Einfluss der Zn-Behandlung (mg/kg Boden) auf das Sprosstrockengewicht (g/Topf) von Casuarina equisetifolia

\begin{tabular}{|l|l|l|l|l|l|l|l|}
\hline mg Zn/kg Boden & 0,0 & 0,5 & 2,5 & 10 & 20 & 40 & GD 5\% \\
\hline STG g/Topf & 1,59 & 1,66 & 1,51 & 1,85 & 1,82 & 1,27 & ns \\
\hline
\end{tabular}

\subsection{Wurzeltrockengewicht (WTG)}

Das Wurzeltrockengewicht schwankte zwischen den einzelnen Behandlungen, signifikante Unterschiede im Vergleich $\mathrm{zu}$ den nicht behandelten Kontrollpflanzen konnten jedoch nicht festgestellt werden (Tab.30). Bei den Zn-Behandlungen von 0,5 bzw. 2,5 mg/kg Boden waren die Wurzeltrockengewichte geringer als die der Kontrollpflanzen. Eine Behandlung mit 10 bzw. $20 \mathrm{mg} \mathrm{Zn/kg}$ Boden führte $\mathrm{zu}$ einem Anstieg der Wurzeltrockengewichte. Alle Werte lagen jedoch niedriger als in der Kontrolle. Das geringste Wurzeltrockengewicht wurde bei Anwendung von $40 \mathrm{mg} \mathrm{Zn} / \mathrm{kg}$ Boden festgestellt. Die höchste $\mathrm{Zn}$-Stufe $40 \mathrm{mg} \mathrm{Zn/kg}$ Boden wirkte mehr toxisch auf die Casuarina-Pflanzen als die anderen Zn-Behandlungen und ebenfalls stärker toxisch als bei den Acacia-Pflanzen.

Tab.30: Einfluss der Zn-Behandlung (mg/kg Boden) auf das Wurzeltrockengewicht (g/Topf) von Casuarina equisetifolia

\begin{tabular}{|l|r|r|r|r|r|r|l|}
\hline mg Zn/kg Boden & 0,0 & 0,5 & 2,5 & 10 & 20 & 40 & GD 5\% \\
\hline WTG g/Topf & 0,25 & 0,19 & 0,18 & 0,24 & 0,22 & 0,14 & $\mathrm{~ns}$ \\
\hline
\end{tabular}




\subsection{Konzentration und Aufnahme von N, P, K, Ca, Mg und Zn im}

Spross

\section{$\mathrm{N}$-Konzentration und -Aufnahme}

Wie aus Tab. 31 hervorgeht, lag die N-Konzentration der Kontrollpflanzen am höchsten.

Erst bei Behandlung mit 10, 20 und $40 \mathrm{mg} \mathrm{Zn/kg} \mathrm{Boden} \mathrm{war} \mathrm{die} \mathrm{N-Konzentration} \mathrm{im}$ Mittel um etwa $20 \%$ signifikant niedriger als die der Kontrollpflanzen. Die N-Aufnahme zeigte keine signifikanten Unterschiede zwischen den Behandlungen.

Tab.31: Einfluss der Zn-Behandlung (mg/kg Boden) auf die Konzentration (mg/g TS) und Aufnahme (mg/Topf) von N im Spross von Casuarina equisetifolia

\begin{tabular}{|l|r|r|}
\hline mg Zn/kg Boden & $\begin{array}{l}\text { N-Konzentration } \\
\text { (mg/g TS) }\end{array}$ & $\begin{array}{l}\text { N-Aufnahme } \\
\text { (mg/Topf) }\end{array}$ \\
\hline 0,0 & 31,9 & 50,1 \\
0,5 & 30,4 & 50,2 \\
2,5 & 29,2 & 44,4 \\
10 & 26,7 & 49,5 \\
20 & 25,0 & 44,8 \\
40 & 25,6 & 32,7 \\
\hline GD 5\% & 2,9 & $\mathrm{~ns}$ \\
\hline
\end{tabular}

\section{P-Konzentration und -Aufnahme}

Die P-Konzentrationen und Aufnahmen im Spross von C. equisetifolia sanken mit zunehmender Zn-Konzentration des Bodens ab (Tab.32). Die signifikant niedrigste PKonzentration wurde bei einer Behandlung mit $10 \mathrm{mg} \mathrm{Zn/kg} \mathrm{Boden} \mathrm{bestimmt,} \mathrm{und} \mathrm{lag} \mathrm{um}$ $32 \%$ niedriger als in der Kontrolle. Nur dieser Unterschied war signifikant. Die PAufnahme der Pflanzen war am geringsten bei der Zn-Behandlung mit $40 \mathrm{mg} / \mathrm{kg}$ Boden und lag um etwa $34 \%$ niedriger als die Kontrolle. 
Tab. 32: Einfluss der Zn-Behandlung (mg/kg Boden) auf die Konzentration (mg/g TS) und Aufnahme (mg/Topf) von P, K, Ca und Mg im Spross von Casuarina equisetifolia

\begin{tabular}{|l|lllll|llll|}
\hline \multirow{2}{*}{ mg Zn/kg Boden } & \multicolumn{7}{|c|}{ Konzentration (mg/g TS) } & \multicolumn{5}{|c}{ Aufnahme (mg/Topf) } \\
\cline { 2 - 9 } & $\mathrm{P}$ & $\mathrm{K}$ & $\mathrm{Ca}$ & $\mathrm{Mg}$ & $\mathrm{P}$ & $\mathrm{K}$ & $\mathrm{Ca}$ & $\mathrm{Mg}$ \\
\hline \multirow{2}{*}{0,0} & 2,5 & 20,2 & 8,0 & 2,1 & 3,8 & 31,7 & 13,2 & 3,3 \\
0,5 & 2,4 & 21,7 & 7,0 & 2,1 & 3,7 & 35,0 & 12,2 & 3,6 \\
2,5 & 2,1 & 21,9 & 6,6 & 2,1 & 3,1 & 32,4 & 10,3 & 3,3 \\
10 & 1,7 & 20,7 & 7,0 & 2,1 & 3,1 & 38,3 & 13,1 & 3,8 \\
20 & 2,1 & 18,3 & 7,3 & 1,8 & 3,5 & 32,6 & 13,4 & 3,4 \\
40 & 2,1 & 17,1 & 7,0 & 1,8 & 2,5 & 21,1 & 9,2 & 2,3 \\
\hline \multirow{2}{*}{ GD $5 \%$} & 0,7 & 2,7 & $\mathrm{~ns}$ & $\mathrm{~ns}$ & 1,1 & $\mathrm{~ns}$ & $\mathrm{~ns}$ & $\mathrm{~ns}$ \\
\hline
\end{tabular}

\section{K-Konzentration und -Aufnahme}

Die K-Konzentration von C. equisetifolia stieg bis zur Zn-Behandlung von $2,5 \mathrm{mg} / \mathrm{kg}$ Boden etwas an (Tab.32). $40 \mathrm{ppm} \mathrm{Zn}$ im Boden führten zu einer signifikanten Verringerung der K-Konzentration in den Pflanzen um $15 \%$ gegenüber der Kontrolle. Bis zu einer Zn-Stufe von $20 \mathrm{mg} / \mathrm{kg}$ Boden zeigten sich keine wesentlichen Unterschiede in der K-Aufnahme der Pflanzen. Bei der Zn-Behandlung von $40 \mathrm{mg} / \mathrm{kg}$ Boden sank die KAufnahme der Pflanzen auf $55 \%$ im Vergleich zur höchsten K-Aufnahme bei 10 ppm ab. Im allgemeinen unterschieden sich die K-Aufnahmen der verschiedenen Zn-Behandlungen nicht signifikant von der Kontrolle.

\section{Ca-Konzentration und -Aufnahme}

Die Ca-Konzentration der Pflanzen sank mit zunehmender Zn-Konzentration des Bodens geringfügig, aber nicht signifikant (Tab.32). Auch die Ca-Aufnahme wurde durch einen Anstieg der Zn-Konzentration im Boden nicht signifikant verändert.

Mg-Konzentration und -Aufnahme

Die Zunahme der Zn-Konzentration im Boden wirkte sich nicht signifikant auf die MgKonzentration der Casuarina-Sprosse aus. Eine Behandlung mit 20 bzw. 40 mg Zn/kg 
Boden führte $\mathrm{zu}$ den niedrigsten Mg-Konzentrationen der Pflanzen (Tab.32). Die MgAufnahme schwankte bis zu einer Zn-Zugabe von 20 mg/kg Boden kaum. Bei der höchsten Zn-Konzentration (40 ppm) sank die Mg-Aufnahme im Spross von C. equisetifolia ab, der Unterschied gegenüber der Kontrolle war jedoch nicht signifikant.

\section{Zn-Konzentration und -Aufnahme}

Mit zunehmender Zn-Konzentration im Boden stieg die Zn-Konzentration und -Aufnahme im Spross der Pflanzen an (Tab.33). Bei den Zn-Behandlungen 10, 20 und $40 \mathrm{mg} / \mathrm{kg}$ Boden waren die Zn-Konzentrationen sowie die Aufnahmen gegenüber den Kontrollpflanzen signifikant erhöht. Die Zn-Konzentration bei der Zn-Stufe von 40 mg/kg wurde auf das 13fache und die Zn-Aufnahme auf das 12fache gegenüber der Kontrolle gesteigert.

Tab. 33: Einfluss der Zn-Behandlung (mg/kg Boden) auf die Konzentration (mg/g TS) und Aufnahme (mg/Topf) von Zn im Spross von Casuarina equisetifolia

\begin{tabular}{|c|c|c|}
\hline mg Zn/kg Boden & $\begin{array}{l}\text { Zn-Konzentration } \\
(\mathrm{mg} / \mathrm{g} \mathrm{TS})\end{array}$ & $\begin{array}{l}\text { Zn-Aufnahme } \\
\text { (mg/Topf) }\end{array}$ \\
\hline 0,0 & 0,05 & 0,07 \\
\hline 0,5 & 0,06 & 0,10 \\
\hline 2,5 & 0,13 & 0,19 \\
\hline 10 & 0,22 & 0,39 \\
\hline 20 & 0,41 & 0,70 \\
\hline 40 & 0,65 & 0,81 \\
\hline GD $5 \%$ & 0,12 & 0,20 \\
\hline
\end{tabular}

\subsection{Zn-Konzentration und -Aufnahme in der Wurzel}

Erwartungsgemäß stieg sowohl die Zn-Konzentration als auch die Zn-Aufnahme der Wurzeln mit zunehmender Zn-Zufuhr zum Boden ab 10 ppm stark an (Tab.34). Die höchste Zn-Aufnahme wurde bei einer Zn-Zugabe 40 mg/kg Boden bestimmt. 
Tab.34: Einfluss der Zn-Behandlung (mg/kg Boden) auf die Konzentration (mg/g TS) und Aufnahme (mg/Topf) von $\mathrm{Zn}$ in der Wurzel von Casuarina equisetifolia

\begin{tabular}{|l|r|r|}
\hline mg Zn/kg Boden & $\begin{array}{l}\text { Zn-Konzentration } \\
\text { (mg/g TS) }\end{array}$ & $\begin{array}{l}\text { Zn-Aufnahme } \\
\text { (mg/Topf) }\end{array}$ \\
\hline 0,0 & 0,09 & 0,02 \\
0,5 & 0,35 & 0,05 \\
2,5 & 0,91 & 0,14 \\
10 & 1,01 & 0,21 \\
20 & 1,65 & 0,32 \\
40 & 1,91 & 0,25 \\
\hline GD 5\% & 0,82 & 0,12 \\
\hline
\end{tabular}

\subsection{Der pH-Wert des Bodens}

Wie bei Acacia saligna sank der pH-Wert des Boden bei Casuarina equisetifolia zum Versuchende ab (Tab.35). Allgemein nahm das pH des Bodens von 7 bis auf 5,2 ab. Die $\mathrm{pH}-$ Werte unterschieden sich nicht signifikant von der Kontrolle.

Tab.35: Einfluss der Zn-Behandlung (mg/kg Boden) auf den pH-Wert im Boden von Casuarina equisetifolia am Ende des Versuches

\begin{tabular}{|l|r|r|l|l|l|l|l|}
\hline $\mathrm{mg} Z n / \mathrm{kg}$ Boden & 0,0 & 0,5 & 2,5 & 10 & 20 & 40 & GD 5\% \\
\hline $\mathrm{pH}$ & 5,3 & 5,5 & 5,4 & 5,5 & 5,2 & 5,3 & $\mathrm{~ns}$ \\
\hline
\end{tabular}




\subsubsection{Cd-Behandlung}

\subsection{Sprosstrockengewicht (STG)}

Bemerkenswert war die schwach positive Wirkung der Cd-Behandlungen 1,25 und 2,5 mg/kg Boden auf das Wachstum der Casuarina-Pflanzen gegenüber der Kontrolle (Tab.36). Erst die höchste Cd-Zugabe $20 \mathrm{mg} / \mathrm{kg}$ Boden wirkte toxisch auf die TS-Bildung im Spross und reduzierte das STG um 71,5 \% im Vergleich zur Kontrolle.

Tab.36: Einfluss der Cd-Behandlung (mg/kg Boden) auf das Sprosstrockengewicht (g/Topf) von Casuarina equisetifolia

\begin{tabular}{|l|r|c|c|c|c|c|l|}
\hline mg Cd/kg Boden & 0,0 & 1,25 & 2,5 & 5 & 10 & 20 & GD 5 \% \\
\hline STG g/Topf & 1,86 & 1,95 & 2,08 & 1,65 & 1,63 & 0,53 & 0,64 \\
\hline
\end{tabular}

\subsection{Wurzeltrockengewicht (WTG)}

Die Wurzeltrockengewichte von Casuarina-Pflanzen nahmen mit steigender Cd-Behandlung bis zu einer Konzentration von $10 \mathrm{mg} \mathrm{Cd} / \mathrm{kg}$ Boden leicht, aber nicht signifikant,zu (Tab.37). Wie beim Spross wirkte die Behandlung mit $20 \mathrm{mg} \mathrm{Cd} / \mathrm{kg}$ Boden stark toxisch auf die Wurzelbildung im Vergleich zu den Kontrollpflanzen.

Tab.37: Einfluss der Cd-Behandlung (mg/kg Boden) auf das Wurzeltrockengewicht (g/Topf) von Casuarina equisetifolia

\begin{tabular}{|l|r|r|r|r|r|r|c|}
\hline mg Cd/kg Boden & 0,0 & 1,25 & 2,5 & 5 & 10 & 20 & GD 5 \% \\
\hline WTG g/Topf & 0,20 & 0,27 & 0,27 & 0,27 & 0,25 & 0,05 & 0,14 \\
\hline
\end{tabular}

\subsection{Konzentration und Aufnahme von $\mathrm{N}, \mathrm{P}, \mathrm{K}, \mathrm{Ca}, \mathrm{Mg}$ und $\mathrm{Cd}$ im Spross}

N-Konzentration und -Aufnahme

Die N-Konzentration und -Aufnahme der Pflanzen wurde bei zunehmender CdKonzentration des Bodens um maximal $17 \%$ bei $20 \mathrm{ppm} \mathrm{Cd}$ gegenüber der Kontrolle verringert (Tab.38). Die behandelten Pflanzen zeigten meistens signifikant verringerte $\mathrm{N}-$ Konzentrationen gegenüber der Kontrolle. Die N-Aufnahme korrelierte nicht mit der TSBildung. Nur bei den beiden höchsten Cd-Stufen war die N-Aufnahme signifikant niedriger verglichen mit der Kontrolle. 
Tab. 38: Einfluss der Cd-Behandlung (mg/kg Boden) auf die Konzentration (mg/g TS) und Aufnahme (mg/Topf) von N im Spross von Casuarina equisetifolia

\begin{tabular}{|l|r|r|}
\hline mg Cd/kg Boden & $\begin{array}{l}\text { N-Konzentration } \\
\text { (mg/g TS) }\end{array}$ & $\begin{array}{l}\text { N-Aufnahme } \\
\text { (mg/Topf) }\end{array}$ \\
\hline 0,0 & 32,9 & 61,2 \\
1,25 & 29,5 & 57,4 \\
2,5 & 29,2 & 60,8 \\
5 & 28,4 & 46,6 \\
10 & 27,3 & 44,6 \\
20 & 27,4 & 14,7 \\
\hline GD 5\% & 3,3 & 18,7 \\
\hline
\end{tabular}

P-Konzentration und -Aufnahme

Die P-Konzentration der Pflanzen wurde durch die steigende Cd-Konzentration des Bodens nicht wesentlich beeinflusst (Tab. 39). Eine Cd-Zugabe zum Boden wirkte sich unterschiedlich negativ auf die P-Aufnahme der Pflanzen aus. Die Unterschiede zwischen den Behandlungen waren nicht signifikant. Die signifikant geringste P-Aufnahme wurde im Vergleich zur Kontrolle bei der höchsten Cd-Zugabe $20 \mathrm{mg} / \mathrm{kg}$ Boden ermittelt. Bei dieser Cd-Stufe wurde ebenfalls das geringste STG festgestellt. 
Tab.39: Einfluss der Cd-Behandlung ( $\mathrm{mg} / \mathrm{kg}$ Boden) auf die Konzentration (mg/g TS) und Aufnahme (mg/Topf) von P, K, Ca und Mg im Spross von Casuarina equisetifolia

\begin{tabular}{|l|llll|lllll|}
\hline \multirow{2}{*}{$\mathrm{mg} \mathrm{Cd} / \mathrm{kg}$ Boden } & \multicolumn{6}{|c|}{ Konzentration (mg/g TS) } & \multicolumn{4}{|c|}{ Aufnahme (mg/Topf) } \\
\cline { 2 - 9 } & $\mathrm{P}$ & $\mathrm{K}$ & $\mathrm{Ca}$ & $\mathrm{Mg}$ & $\mathrm{P}$ & $\mathrm{K}$ & $\mathrm{Ca}$ & $\mathrm{Mg}$ \\
\hline 0,0 & 2,5 & 24,7 & 7,3 & 1,9 & 4,3 & 45,2 & 13,6 & 3,5 \\
1,25 & 1,5 & 20,8 & 8,2 & 2,1 & 3,0 & 39,9 & 16,2 & 4,1 \\
2,5 & 2,3 & 18,8 & 7,0 & 1,9 & 4,8 & 39,5 & 14,7 & 4,0 \\
5 & 2,3 & 18,5 & 6,9 & 1,9 & 3,3 & 29,1 & 12,1 & 3,3 \\
10 & 2,4 & 18,0 & 6,9 & 1,6 & 3,6 & 29,2 & 11,8 & 2,8 \\
20 & 2,5 & 17,6 & 5,9 & 1,3 & 1,4 & 9,4 & 3,1 & 0,7 \\
\hline \multirow{2}{*}{$\mathrm{GD} 5 \%$} & $\mathrm{Ns}$ & 4,2 & $\mathrm{~ns}$ & 0,5 & 1,3 & 10,7 & 7,5 & 1,8 \\
\hline
\end{tabular}

K-Konzentration und -Aufnahme

Die K-Konzentration und -Aufnahme von Casuarina-Pflanzen sank mit zunehmender CdZufuhr zum Boden von 2,5 bis $20 \mathrm{mg} / \mathrm{kg}$ Boden signifikant im Vergleich zu den Kontrollpflanzen ab (Tab.39). Die K-Aufnahme im Spross lag ab der Cd-Stufe $5 \mathrm{mg} / \mathrm{kg}$ Boden signifikant niedriger als in der Kontrollvariante. Die niedrigste K-Konzentration bzw. -Aufnahme wurde bei der höchsten Cd-Behandlung $20 \mathrm{mg} \mathrm{Cd} / \mathrm{kg}$ Boden festgestellt.

\section{Ca-Konzentration und -Aufnahme}

Wie aus Tab. 39 hervorgeht, stieg die Ca-Konzentration der Pflanzen bei der Behandlung mit 1,25 mg Cd/kg Boden an. Ein weiterer Anstieg der Cd-Konzentration im Boden führte zu sinkenden Ca-Konzentrationen. Die niedrigste Ca-Aufnahme wurde bei der höchsten Cd-Behandlung $20 \mathrm{mg} \mathrm{Cd} / \mathrm{kg}$ Boden gefunden, wo das Wachstum am stärksten beeinträchtigt wurde.

Mg-Konzentration und -Aufnahme

Die Zunahme der Konzentration von $\mathrm{Cd}$ im Boden verringerte die Mg-Konzentrationen und -Aufnahmen der Pflanzen (Tab.39). Entsprechend dem Wachstum wurde die signifikant geringste Mg-Konzentration und -Aufnahme bei der höchsten Cd-Behandlung $20 \mathrm{mg} / \mathrm{kg}$ Boden bestimmt. 
Cd-Konzentration und -Aufnahme

Durch die Steigerung der Cd-Konzentration im Boden nahm sowohl die Cd-Konzentration als auch die Cd-Aufnahme im Spross der Pflanzen zu (Tab.40). Diese Zunahme war bei den Cd-Stufen 5, 10 und $20 \mathrm{mg} / \mathrm{kg}$ Boden signifikant gegenüber der Kontrolle. Die Behandlung mit $20 \mathrm{mg} \mathrm{Cd} / \mathrm{kg}$ Boden führte zu einer drastischen Steigerung der CdKonzentration und -Aufnahme der dadurch beschädigten Pflanzen. Diese Cd-Stufe beeinträchtigte das Wachstum der Casuarina-Pflanzen stärker als das der Acacia-Pflanzen.

Tab.40: Einfluss der Cd-Behandlung (mg/kg Boden) auf die Konzentration (mg/g TS) und Aufnahme (mg/Topf) von Cd im Spross von Casuarina equisetifolia

\begin{tabular}{|l|r|r|}
\hline mg Cd/kg Boden & $\begin{array}{l}\text { Cd-Konzentration } \\
\text { (mg/g TS) }\end{array}$ & $\begin{array}{l}\text { Cd-Aufnahme } \\
\text { (mg/Topf) }\end{array}$ \\
\hline 0,0 & 0,00 & 0,00 \\
1,25 & 0,01 & 0,02 \\
2,5 & 0,02 & 0,03 \\
5 & 0,04 & 0,06 \\
10 & 0,06 & 0,09 \\
20 & 0,13 & 0,07 \\
\hline GD 5\% & 0,04 & 0,05 \\
\hline
\end{tabular}

\subsection{Cd-Konzentration und -Aufnahme in der Wurzel}

Durch die Zufuhr von Cd zum Boden akkumulierten die Wurzeln bei jeder Cd-Stufe hohe Mengen an diesem Schwermetall (Tab.41). Diese Mengen waren unvergleichlich höher als die der Sprosse. Die Cd-Aufnahmen der Wurzeln der verschiedenen Cd-Behandlungen lagen mehr oder weniger wegen des gehemmten Wurzelwachstums auf einem ähnlichen Niveau wie bei den Sprossen. 
Tab.41: Einfluss der Cd-Behandlung (mg/kg Boden) auf die Konzentration (mg/g TS) und Aufnahme (mg/Topf) von Cd in der Wurzel von Casuarina equisetifolia

\begin{tabular}{|l|r|r|}
\hline mg Cd/kg Boden & $\begin{array}{l}\text { Cd-Konzentration } \\
\text { (mg/g TS) }\end{array}$ & $\begin{array}{l}\text { Cd-Aufnahme } \\
\text { (mg/Topf) }\end{array}$ \\
\hline 0,0 & 0,00 & 0,00 \\
1,25 & 0,19 & 0,05 \\
2,5 & 0,21 & 0,06 \\
5 & 0,32 & 0,07 \\
10 & 0,41 & 0,08 \\
20 & 1,27 & 0,07 \\
\hline GD 5\% & 0,27 & 0,00 \\
\hline
\end{tabular}

\subsection{Der $\mathrm{pH}-$ Wert des Bodens}

Wie bei der Zn-Zufuhr zum Boden sank der Boden-pH-Wert von 7 auf 5,1 am Ende der Vegetationsperiode ab (Tab.42). Jedoch stieg der $\mathrm{pH}$-Wert durch die Cd-Behandlung, anders als bei der Zn-Behandlung, an. Eine Behandlung mit 2,5; 5 oder $10 \mathrm{mg} \mathrm{Cd} / \mathrm{kg}$ Boden führte zu einem signifikanten Anstieg des Boden-pH-Wertes im Vergleich zur Kontrolle.

Tab. 42: Einfluss der Cd-Behandlung (mg/kg Boden) auf den pH-Wert im Boden von Casuarina equisetifolia am Ende des Versuches

\begin{tabular}{|l|c|c|l|l|l|l|l|}
\hline $\mathrm{mg} \mathrm{Cd} / \mathrm{kg}$ Boden & 0,0 & 1,25 & 2,5 & 5 & 10 & 20 & GD 5\% \\
\hline $\mathrm{pH}$ & 5,1 & 5,3 & 5,6 & 5,6 & 5,8 & 5,3 & 0,4 \\
\hline
\end{tabular}




\subsubsection{Pb-Behandlung}

\subsection{Sprosstrockengewicht (STG)}

Wie aus Tab. 43 hervorgeht, nahm das STG von C. equisetifolia mit ansteigenden $\mathrm{Pb}$ Konzentrationen (in Form von stickstoffhaltigem Bleinitrat) im Boden zu. Bei allen Behandlungsstufen wurden signifikante Unterschiede gegenüber den Kontrollpflanzen festgestellt. Die $\mathrm{Pb}$-Konzentration von $10 \mathrm{mg} / \mathrm{kg}$ Boden wirkte sich am günstigsten auf das Sprosstrockengewicht der Pflanzen aus. Bei weiter ansteigenden $\mathrm{Pb}$-Behandlungen bis 20 und $40 \mathrm{mg} / \mathrm{kg}$ Boden nahmen die Sprosstrockengewichte ab, alle Werte blieben jedoch signifikant über der Kontrolle.

Tab.43: Einfluss der $\mathrm{Pb}$-Behandlung (mg/kg Boden) auf das Sprosstrockengewicht (g/Topf) von Casuarina equisetifolia

\begin{tabular}{|l|l|c|c|c|c|c|l|}
\hline $\mathrm{mg} \mathrm{Pb} / \mathrm{kg}$ Boden & 0,0 & 2,5 & 5 & 10 & 20 & 40 & GD 5 \% \\
\hline STG g/Topf & 1,37 & 1,98 & 2,11 & 2,15 & 1,95 & 1,68 & 0,23 \\
\hline
\end{tabular}

\subsection{Wurzeltrockengewicht (WTG)}

Das Wurzeltrockengewicht von C. equisetifolia zeigte bei der Pb-Konzentration $2,5 \mathrm{mg} / \mathrm{kg}$ Boden einen signifikanten Anstieg im Vergleich $\mathrm{zu}$ den Kontrollpflanzen (Tab.44). Die weiteren $\mathrm{Pb}$-Konzentrationserhöhungen wirkten sich nicht signifikant auf das Wurzeltrockengewicht der Pflanzen aus.

Tab.44: Einfluss der Pb-Behandlung (mg/kg Boden) auf das Wurzeltrockengewicht (g/Topf) von Casuarina equisetifolia

\begin{tabular}{|l|l|c|c|c|c|c|l|}
\hline mg Pb/kg Boden & 0,0 & 2,5 & 5 & 10 & 20 & 40 & \\
\hline WTG g/Topf & 0,21 & 0,31 & 0,24 & 0,25 & 0,23 & 0,22 & 0,10 \\
\hline
\end{tabular}


5.1.2.3.3 Konzentration und Aufnahme von N, P, K, Ca, Mg und Pb im Spross

N-Konzentration und -Aufnahme

Eine Zunahme der $\mathrm{Pb}$-Konzentration (in Form von $\left.\mathrm{Pb}\left(\mathrm{NO}_{3}\right)_{2}\right)$ im Boden wirkte sich nicht auf die N-Konzentration im Spross von C. equisetifolia aus (Tab.45). Die N-Aufnahme der Pflanzen stieg mit zunehmender $\mathrm{Pb}$-Konzentration entsprechend dem Wachstum an. Signifikante Unterschiede zur Kontrolle wurden bei einer Pb-Behandlung von 2,5; 5; 10 und $20 \mathrm{mg} / \mathrm{kg}$ Boden festgestellt.

Tab.45: Einfluss der Pb-Behandlung (mg/kg Boden) auf die Konzentration (mg/g TS) und Aufnahme (mg/Topf) von N im Spross von Casuarina equisetifolia

\begin{tabular}{|c|r|r|}
\hline mg Pb/kg Boden & $\begin{array}{c}\text { N-Konzentration } \\
\text { (mg/g TS) }\end{array}$ & $\begin{array}{l}\text { N-Aufnahme } \\
\text { (mg/Topf) }\end{array}$ \\
\hline 0,0 & 26,8 & 36,8 \\
2,5 & 29,0 & 57,3 \\
5 & 24,1 & 51,0 \\
10 & 27,1 & 58,2 \\
20 & 25,4 & 49,7 \\
40 & 26,0 & 43,7 \\
\hline GD 5\% & ns & 9,7 \\
\hline
\end{tabular}

P-Konzentration und -Aufnahme

Die P-Konzentration der Casuarina-Pflanzen wurde durch eine Zunahme der PbKonzentration im Boden nicht signifikant beeinflusst (Tab.46). Die P-Aufnahme wurde mit steigender Pb-Konzentration im Boden erhöht. Bei einer Pb-Behandlung von $20 \mathrm{mg} / \mathrm{kg}$ Boden wiesen die Pflanzen eine signifikant höhere P-Aufnahme als die Kontrollpflanzen auf. 
Tab.46: Einfluss der $\mathrm{Pb}$-Behandlung (mg/kg Boden) auf die Konzentration (mg/g TS) und Aufnahme (mg/Topf) von P, K, Ca und Mg im Spross von Casuarina equisetifolia

\begin{tabular}{|l|cccc|ccccc|}
\hline \multirow{2}{*}{ mg Pb/kg Boden } & \multicolumn{4}{|c|}{ Konzentration (mg/g TS) } & \multicolumn{4}{l|}{ Aufnahme (mg/Topf) } \\
\cline { 2 - 9 } & $\mathrm{P}$ & $\mathrm{K}$ & $\mathrm{Ca}$ & $\mathrm{Mg}$ & $\mathrm{P}$ & $\mathrm{K}$ & $\mathrm{Ca}$ & $\mathrm{Mg}$ \\
\hline 0,0 & 1,9 & 18,6 & 5,8 & 2,2 & 2,5 & 25,4 & 7,8 & 3,0 \\
2,5 & 2,0 & 18,8 & 5,6 & 2,2 & 3,9 & 37,2 & 11,0 & 4,3 \\
5 & 1,8 & 15,9 & 6,3 & 2,0 & 3,9 & 33,5 & 13,3 & 4,1 \\
10 & 1,7 & 16,8 & 6,2 & 2,1 & 3,7 & 36,2 & 13,2 & 4,6 \\
20 & 2,6 & 16,1 & 6,2 & 3,0 & 5,1 & 31,4 & 11,8 & 5,7 \\
40 & 2,1 & 16,8 & 6,5 & 2,9 & 3,5 & 28,2 & 10,9 & 4,9 \\
\hline \multirow{2}{*}{$\mathrm{GD} \mathrm{5 \%}$} & 0,7 & 2,3 & $\mathrm{~ns}$ & 0,4 & 1,8 & 6,3 & 2,1 & 0,7 \\
\hline
\end{tabular}

\section{K-Konzentration und -Aufnahme}

Die K-Konzentration der Casuarina-Pflanzen ging mit zunehmender Pb-Konzentration im Boden leicht zurück (Tab.46). Bei einer Pb-Behandlung mit $5 \mathrm{mg} / \mathrm{kg}$ Boden zeigten die Pflanzen eine signifikant niedrige K-Konzentration als die Kontrollpflanzen. Die KAufnahme der behandelten Pflanzen nahm mit steigender Pb-Konzentration im Boden zu. Signifikante Unterschiede gegenüber den nicht behandelten Kontrollpflanzen wurden bei einer Gabe von 2,5; 5 oder $10 \mathrm{mg} \mathrm{Pb} / \mathrm{kg}$ Boden ermittelt.

\section{Ca-Konzentration und -Aufnahme}

Die Ca-Konzentration der Casuarina-Pflanzen stieg mit zunehmender Pb-Konzentration im Boden geringfügig an (Tab.46). Die behandelten Casuarina-Pflanzen zeigten eine signifikant höhere $\mathrm{Ca}$-Aufnahme mit steigender $\mathrm{Pb}$-Konzentration des Bodens. Die höchste $\mathrm{Ca}$-Aufnahme wurde bei der Pb-Behandlung $5 \mathrm{mg} / \mathrm{kg}$ Boden festgestellt. Bei weiterer Erhöhung der $\mathrm{Pb}$-Konzentration ging die Ca-Aufnahme der Pflanzen zurück, war jedoch gegenüber den Kontrollpflanzen signifikant erhöht.

Mg-Konzentration und -Aufnahme

Die Mg-Konzentration von C. equisetifolia blieb mit steigender Pb-Konzentration bis zu 10 mg/kg Boden unverändert (Tab.46). Die Behandlungen mit 20 bzw. 40 mg Pb/kg Boden 
führten $\mathrm{zu}$ einem signifikanten Anstieg der Mg-Konzentrationen um etwa $35 \% \mathrm{im}$ Vergleich zu den Kontrollpflanzen. Die Mg-Aufnahme der Pflanzen war bei allen Behandlungsstufen signifikant höher als die der Kontrollpflanzen. Die höchste MgAufnahme wurde bei der $\mathrm{Pb}$-Stufe $20 \mathrm{mg} / \mathrm{kg}$ Boden gefunden. Die Behandlung mit $40 \mathrm{mg} \mathrm{Pb} / \mathrm{kg}$ Boden führte $\mathrm{zu}$ einer entsprechend dem Wachstum geringeren $\mathrm{Mg}$ Aufnahme der Pflanzen als bei der Pb-Stufe $20 \mathrm{mg} / \mathrm{kg}$ Boden.

\section{$\mathrm{Pb}$-Konzentration und -Aufnahme}

Die Pb-Konzentration bzw. -Aufnahme im Spross der Casuarina-Pflanzen wurde besonders bei den höheren Behandlungsstufen im Vergleich zu Kontrollpflanzen erhöht (Tab. 47). Signifikante Unterschiede in der Pb-Konzentration wurde bei Behandlung mit 20 bzw. $40 \mathrm{mg} \mathrm{Pb} / \mathrm{kg}$ Boden ermittelt. Die Pb-Aufnahme der Pflanzen lag bei allen $\mathrm{Pb}$ Behandlungsstufen signifikant höher als in den Kontrollpflanzen. Die höchsten Werte der $\mathrm{Pb}$-Konzentration bzw. -Aufnahme wurde bei der letzten Pb-Stufe $40 \mathrm{mg} / \mathrm{kg}$ Boden, Gegenüber der Kontrolle stieg die Pb-Konzentration auf das 4fache, die Pb-Aufnahme auf das 6 fache.

Tab. 47: Einfluss der $\mathrm{Pb}-$ Behandlung (mg/kg Boden) auf die Konzentration (mg/g TS) und Aufnahme (mg/Topf) von Pb im Spross von Casuarina equisetifolia

\begin{tabular}{|l|r|r|}
\hline mg Pb/kg Boden & $\begin{array}{l}\text { Pb-Konzentration } \\
(\mathrm{mg} / \mathrm{g} \text { TS })\end{array}$ & $\begin{array}{l}\text { Pb-Aufnahme } \\
\text { (mg/Topf })\end{array}$ \\
\hline 0,0 & 0,01 & 0,01 \\
2,5 & 0,01 & 0,02 \\
5 & 0,01 & 0,02 \\
10 & 0,01 & 0,03 \\
20 & 0,02 & 0,04 \\
40 & 0,04 & 0,06 \\
\hline GD $5 \%$ & 0,01 & 0,00 \\
\hline
\end{tabular}

\subsection{Pb-Konzentration und -Aufnahme in der Wurzel}

Mit steigender Pb-Konzentration im Boden wiesen die Casuarina-Pflanzen eine drastisch erhöhte Pb-Akkumulation in ihren Wurzeln auf (Tab.48). Die Pb-Aufnahme der Wurzeln 
nahm mit zunehmender Pb-Konzentration des Bodens zu. Bei einer Behandlung mit 20 bzw. $40 \mathrm{mg} \mathrm{Pb/kg} \mathrm{Boden} \mathrm{wiesen} \mathrm{die} \mathrm{Casuarina} \mathrm{-Wurzeln} \mathrm{eine} \mathrm{signifikant} \mathrm{erhöhte} \mathrm{Pb}$ Aufnahme auf, was der TS-Bildung nicht entsprach.

Tab.48: Einfluss der Pb-Behandlung (mg/kg Boden) auf die Konzentration (mg/g TS) und Aufnahme (mg/Topf) von Pb in der Wurzel von Casuarina equisetifolia

\begin{tabular}{|l|r|r|}
\hline mg Pb/kg Boden & $\begin{array}{l}\text { Pb-Konzentration } \\
\text { (mg/g TS) }\end{array}$ & $\begin{array}{l}\text { Pb-Aufnahme } \\
\text { (mg/Topf) }\end{array}$ \\
\hline 0,0 & 0,00 & 0,00 \\
2,5 & 0,06 & 0,02 \\
5 & 0,07 & 0,02 \\
10 & 0,20 & 0,05 \\
20 & 0,42 & 0,10 \\
40 & 0,82 & 0,17 \\
\hline GD 5\% & 0,29 & 0,07 \\
\hline
\end{tabular}

\subsection{Der $\mathrm{pH}-$ Wert des Bodens}

Die Zunahme der Pb-Konzentration im Boden wirkte sich nicht signifikant auf den $\mathrm{pH}$ Wert des Bodens aus (Tab.49). Generell sank der Boden-pH von 7 bis auf 5,2 am Ende der Vegetationperiode $\mathrm{ab}$.

Tab. 49: Einfluss der Pb-Behandlung (mg/kg Boden) auf den $\mathrm{pH}-$ Wert im Boden von Casuarina equisetifolia am Ende des Versuches

\begin{tabular}{|l|c|c|c|c|c|c|l|}
\hline $\mathrm{mg} \mathrm{Pb} / \mathrm{kg}$ Boden & 0,0 & 2,5 & 5 & 10 & 20 & 40 & GD 5\% \\
\hline $\mathrm{pH}$ & 5,3 & 5,2 & 5,4 & 5,3 & 5,5 & 5,4 & $\mathrm{~ns}$ \\
\hline
\end{tabular}




\subsubsection{Cupressus sempervirens}

\subsubsection{Zn-Behandlung}

\subsection{Sprosstrockengewicht (STG)}

Das Sprosstrockengewicht von C. sempervirens nahm mit steigender Zn-Konzentration im Boden meist zu (Tab.50). Das signifikant höchste STG gegenüber der Kontrolle trat bei der Behandlung mit $10 \mathrm{mg} \mathrm{Zn} / \mathrm{kg}$ Boden auf. Eine weitere Steigerung der Zn-Zugabe auf 20 bzw. 40 ppm Zn führte zu einer Abnahme des Sprosstrockengewichtes. Die CupressusPflanzen reagierten auf beide Zn-Stufen empfindlicher als die Acacia- (Tab.8) und Casuarina-Pflanzen (Tab.29).

Tab.50: Einfluss der Zn-Behandlung (mg/kgBoden) auf das Sprosstrockengewicht (g/Topf) von Cupressus sempervirens

\begin{tabular}{|l|l|l|l|l|l|l|l|}
\hline mg Zn/kg Boden & 0,0 & 0,5 & 2,5 & 10 & 20 & 40 & GD 5\% \\
\hline STG g/Topf & 1,66 & 1,78 & 1,96 & 2,04 & 1,41 & 1,18 & 0,38 \\
\hline
\end{tabular}

\subsection{Wurzeltrockengewicht (WTG)}

Wie der STG, nahm das Wurzeltrockengewicht der behandelten Pflanzen mit steigender Zn-Behandlung bis $10 \mathrm{mg} / \mathrm{kg}$ Boden zu (Tab.51). Das höchste WTG bei der Zn-Stufe $10 \mathrm{mg} / \mathrm{kg}$ lag um $32 \%$ höher als in der Kontrolle. Die weitere Erhöhung der ZnKonzentration im Boden wirkte sich negativ auf das Wurzeltrockengewicht der Pflanzen aus. Bei der höchsten Zn-Stufe $40 \mathrm{mg} / \mathrm{kg}$ Boden wurde eine signifikante Verringerung des Wurzeltrockengewichtes gegenüber der Kontrolle ermittelt.

Tab.51: Einfluss der Zn-Behandlung (mg/kg Boden) auf das Wurzeltrockengewicht (g/Topf) von Cupressus sempervirens

\begin{tabular}{|l|r|r|r|r|r|r|l|}
\hline mg Zn/kg Boden & 0,0 & 0,5 & 2,5 & 10 & 20 & 40 & GD 5 \% \\
\hline WTG g/Topf & 0,41 & 0,45 & 0,50 & 0,54 & 0,30 & 0,25 & 0,13 \\
\hline
\end{tabular}




\subsection{Konzentration und Aufnahme von N, P, K, Ca, Mg und Zn im}

\section{Spross}

\section{N-Konzentration und -Aufnahme}

Die zunehmende Konzentration von $\mathrm{Zn}$ im Boden führte $\mathrm{zu}$ einer erhöhten $\mathrm{N}$ Konzentration in den Pflanzen (Tab.52). Bei 10 bzw. $20 \mathrm{mg} \mathrm{Zn/kg} \mathrm{Boden} \mathrm{akkumulierten}$ die Pflanzen signifikant mehr $\mathrm{N}$ als die Kontrollpflanzen. $40 \mathrm{ppm} \mathrm{Zn}$ wirkte sich entsprechend dem Wachstum negativ auf die NKonzentration der Pflanzen aus. Die NAufnahme entsprach dem Wachstum. Bei Behandlung mit 2,5 bzw. $10 \mathrm{mg} \mathrm{Zn} / \mathrm{kg}$ Boden zeigten die Pflanzen eine signifikant erhöhte $\mathrm{N}$-Aufnahme im Vergleich $\mathrm{zu}$ den Kontrollpflanzen. Die Behandlung mit $40 \mathrm{mg} \mathrm{Zn/kg} \mathrm{Boden} \mathrm{führte} \mathrm{zu} \mathrm{einer} \mathrm{verringerten} \mathrm{N}$ Aufnahme der Pflanzen, die aber im Gegensatz zum STG nicht signifikant niedriger als die N-Aufnahme der Kontrollvariante war.

Tab.52: Einfluss der Zn-Behandlung (mg/kg Boden) auf die Konzentration ( mg/g TS) und Aufnahme ( $\mathrm{mg} /$ Topf) von N im Spross von Cupressus sempervirens

\begin{tabular}{|l|r|r|}
\hline mg Zn/kg Boden & $\begin{array}{l}\text { N-Konzentration } \\
\text { (mg/g TS })\end{array}$ & $\begin{array}{l}\text { N-Aufnahme } \\
\text { (mg/Topf) }\end{array}$ \\
\hline 0,0 & 12,5 & 19,7 \\
0,5 & 14,3 & 25,4 \\
2,5 & 15,2 & 30,1 \\
10 & 16,4 & 33,8 \\
20 & 16,7 & 24,1 \\
40 & 12,1 & 14,6 \\
\hline GD $5 \%$ & 3,2 & 10,4 \\
\hline
\end{tabular}

\section{P-Konzentration und -Aufnahme}

Die Zn-Behandlung wirkte sich nur unwesentlich auf die P-Konzentration der CupressusPflanzen aus (Tab.53). Die erhöhten Zn-Zugaben zum Boden auf 20 bzw. 40 mg/kg Boden führten zu geringeren P-Konzentrationen der Pflanzen. Die P-Aufnahme stieg mit der Zunahme der Zn-Gabe zum Boden bis zu $10 \mathrm{mg} / \mathrm{kg}$ Boden entsprechend dem Wachstum an. Diese Steigerung der P-Aufnahme bei den erwähnten Zn-Stufen war nicht signifikant 
gegenüber der Kontrolle. Weitere Erhöhungen der Zn-Gaben führten, dem Wachstum entsprechend, zu einer verringerten P-Aufnahme den Pflanzen, die bei der höchsten ZnStufe $40 \mathrm{mg} / \mathrm{kg}$ Boden signifikant niedriger als in der Kontrolle lag.

Tab.53: Einfluss der Zn-Behandlung (mg/kg Boden) auf die Konzentration (mg/g TS) und Aufnahme (mg/Topf) von P, K, Ca und Mg im Spross von Cupressus sempervirens

\begin{tabular}{|c|c|c|c|c|c|c|c|c|}
\hline \multirow[t]{2}{*}{ mg Zn/kg Boden } & \multicolumn{4}{|c|}{ Konzentration (mg/g TS) } & \multicolumn{4}{|c|}{ Aufnahme (mg/Topf) } \\
\hline & $\mathrm{P}$ & $\mathrm{K}$ & $\mathrm{Ca}$ & $\mathrm{Mg}$ & $\mathrm{P}$ & $\mathrm{K}$ & $\mathrm{Ca}$ & $\mathrm{Mg}$ \\
\hline 0,0 & 2,1 & 11,3 & 4,7 & 1,1 & 3,4 & 18,6 & 7,8 & 1,7 \\
\hline 0,5 & 2,2 & 13,6 & 5,4 & 1,4 & 4,0 & 24,1 & 9,7 & 2,5 \\
\hline 2,5 & 2,1 & 15,2 & 5,1 & 1,4 & 4,2 & 29,7 & 10,0 & 2,6 \\
\hline 10 & 2,1 & 13,5 & 3,2 & 1,1 & 4,4 & 27,8 & 6,4 & 2,3 \\
\hline 20 & 1,6 & 14,1 & 4,9 & 1,3 & 2,2 & 19,6 & 6,7 & 1,8 \\
\hline 40 & 1,2 & 9,0 & 5,2 & 1,2 & 1,4 & 10,9 & 5,9 & 1,4 \\
\hline GD $5 \%$ & 0,5 & 2,8 & ns & ns & 1,3 & 7,2 & ns & 0,6 \\
\hline
\end{tabular}

K-Konzentration und -Aufnahme

Nur die Behandlung mit $40 \mathrm{ppm} \mathrm{Zn}$ wirkte sich negativ auf die K-Konzentration der Pflanzen aus (Tab.53). Die K-Konzentration nahm hier um $20 \%$ gegenüber der Kontrolle ab. Bis zur Zn-Zugabe von $20 \mathrm{mg} / \mathrm{kg}$ Boden nahmen die Pflanzen mehr Kalium auf, signifikante Unterschiede ergaben sich aber nur bei 2,5 und $10 \mathrm{mg} \mathrm{Zn} / \mathrm{kg}$ Boden. Die weitere Steigerung der Zn-Zugabe führte zu einer signifikant verringerten K-Aufnahme der Pflanzen. Die K-Aufnahme wurde bei der höchsten Zn-Konzentration gegenüber den Kontrollpflanzen um $41 \%$ signifikant reduziert.

Ca-Konzentration und -Aufnahme

Die Ca-Konzentration im Spross der Cupressus-Pflanzen wurde durch die Zn-Zugaben unwesentlich verändert (Tab.53). Die Ca-Aufnahme nahm bis zur Zn-Behandlung mit 2,5 mg/kg Boden zu, sank jedoch bei weiterer Zufuhr von $\mathrm{Zn}$ ab. Bei allen Zn-Stufen waren die Unterschiede gegenüber den Kontrollpflanzen nicht signifikant. 
Mg-Konzentration und -Aufnahme

Die Mg-Konzentration der Zn-Stufen lag meistens nur geringfügig höher als die der Kontrolle (Tab.53). Die Mg-Aufnahme wurde durch steigende Zn-Behandlung bis zu $10 \mathrm{ppm}$ signifikant erhöht. Weiter zunehmende Zn-Konzentrationen in Boden führten zu absinkenden Mg-Aufnahmen der Pflanzen, auf das Niveau der Kontroll-Variante.

Zn-Konzentration und -Aufnahme

Insbesondere bei den höheren Zn-Zugaben wurde die Zn-Konzentration bzw. -Aufnahme im Spross der Cupressus-Pflanzen im Vergleich zur Kontrolle sehr stark gesteigert (Tab.54). Zum Beispiel ergab die höchste Zn-Stufe $40 \mathrm{mg} \mathrm{Zn/kg} \mathrm{Boden} \mathrm{eine} \mathrm{Zn-}$ Konzentration im Spross, die auf das 41fache, bzw. eine Zn-Aufnahme, die auf das 31 fache erhöht wurde.

Tab.54: Einfluss der Zn-Behandlung (mg/kg Boden) auf die Konzentration (mg/g TS) und Aufnahme (mg/Topf) von $\mathrm{Zn}$ im Spross von Cupressus sempervirens

\begin{tabular}{|l|r|r|}
\hline mg Zn/kg Boden & $\begin{array}{l}\text { Zn-Konzentration } \\
\text { (mg/g TS) }\end{array}$ & $\begin{array}{l}\text { Zn-Aufnahme } \\
\text { (mg/Topf) }\end{array}$ \\
\hline 0,0 & 0,02 & 0,03 \\
0,5 & 0,03 & 0,06 \\
2,5 & 0,06 & 0,12 \\
10 & 0,19 & 0,39 \\
20 & 0,43 & 0,57 \\
40 & 0,82 & 0,92 \\
\hline GD 5\% & 0,26 & 0,22 \\
\hline
\end{tabular}

\subsection{Zn-Konzentration und -Aufnahme in der Wurzel}

Die Zn-Konzentration in den Wurzeln zeigte eine ähnliche Tendenz wie die der Sprosse (Tab.55). Sie stieg mit zunehmender Zn-Konzentration im Boden noch stärker als im Spross. Die höchste Zn-Aufnahme betrug bei der Zn-Stufe $10 \mathrm{mg} / \mathrm{kg}$ Boden mehr als das 11 fache der Kontrolle. Die Zn-Aufnahme der Wurzeln stieg bis zur Behandlung mit 10 pm an und veränderte sich bei zunehmender Zn-Zugabe nicht mehr signifikant. 
Tab.55: Einfluss der Zn-Behandlung (mg/kg Boden) auf die Konzentration (mg/g TS) und Aufnahme (mg/Topf) von $\mathrm{Zn}$ in der Wurzel von Cupressus sempervirens

\begin{tabular}{|l|r|r|}
\hline mg Zn/kg Boden & $\begin{array}{l}\text { Zn-Konzentration } \\
\text { (mg/g TS) }\end{array}$ & $\begin{array}{l}\text { Zn-Aufnahme } \\
\text { (mg/Topf) }\end{array}$ \\
\hline 0,0 & 0,09 & 0,04 \\
0,5 & 0,16 & 0,08 \\
2,5 & 0,33 & 0,16 \\
10 & 0,86 & 0,45 \\
20 & 1,44 & 0,42 \\
40 & 1,47 & 0,40 \\
\hline GD 5\% & 0,59 & 0,22 \\
\hline
\end{tabular}

\subsection{Der $\mathrm{pH}-$ Wert des Boden}

Der pH-Wert im Boden bei der Cupressus-Pflanzen zeigte eine sehr ähnliche Tendenz wie bei A. saligna und C. equisetifolia (Tab.56). Der Ausgangs-pH sank von 7 bis auf 4.7 am Ende des Versuchs ab. Signifikante Unterschiede des pH-Wertes konnten durch die Konzentrationssteigerung von $\mathrm{Zn}$ im Boden nicht festgestellt werden.

Tab. 56: Einfluss der Zn-Behandlung (mg/kg Boden) auf den $\mathrm{pH}-$ Wert im Boden von Cupressus sempervirens am Ende des Versuches

\begin{tabular}{|l|r|r|r|r|r|r|l|}
\hline $\mathrm{mg} Z \mathrm{Zn} / \mathrm{kg}$ Boden & 0,0 & 0,5 & 2,5 & 10 & 20 & 40 & GD 5\% \\
\hline $\mathrm{pH}$ & 4,7 & 4,9 & 4,8 & 4,9 & 4,9 & 4,8 & $\mathrm{nS}$ \\
\hline
\end{tabular}




\subsubsection{Cd-Behandlung}

\subsection{Sprosstrockengewicht (STG)}

Im allgemeinen sank das STG mit steigender Cd-Konzentration im Boden ab (Tab.57). Das STG nahm bei der Behandlung mit 2,5 ppm Cd gegenüber der Kontrolle zu, allerdings nicht signifikant. Die Erhöhung der Cd-Zugabe über $10 \mathrm{ppm}$ führte zu einer deutlichen und signifikanten Beeinträchtigung der TS-Bildung im Spross. Die beiden höchsten CdKonzentrationen beeinträchtigten das Wachstum der Cupressus-Pflanzen am stärksten.

Tab.57: Einfluss der Cd-Behandlung (mg/kg Boden) auf das Sprosstrockengewicht (g/Topf) von Cupressus sempervirens

\begin{tabular}{|l|r|r|r|r|r|r|l|}
\hline $\mathrm{mg} \mathrm{Cd} / \mathrm{kg}$ Boden & 0,0 & 1,25 & 2,5 & 5 & 10 & 20 & GD 5 \% \\
\hline STG g/Topf & 1,25 & 1,19 & 1,53 & 1,10 & 0,81 & 0,64 & 0,35 \\
\hline
\end{tabular}

\subsection{Wurzeltrockengewicht (WTG)}

Die TS-Bildung in den Wurzeln zeigte ähnliche Tendenzen wie im Spross, aber auf einem niedrigeren Niveau (Tab. 58). Die schädigende Wirkung der beiden höchsten Cd-Stufen war ausgeprägter als beim Spross.

Tab.58: Einfluss der Cd-Behandlung (mg/kg Boden) auf das Wurzeltrockengewicht (g/Topf) von Cupressus sempervirens

\begin{tabular}{|l|l|l|l|l|l|l|l|}
\hline mg Cd/kg Boden & 0,0 & 1,25 & 2,5 & 5 & 10 & 20 & GD 5\% \\
\hline WTG g/Topf & 0,30 & 0,29 & 0,33 & 0,24 & 0,15 & 0,12 & 0,11 \\
\hline
\end{tabular}

\subsection{Konzentration und Aufnahme von N, P, K, Ca, Mg und Cd im Spross}

N-Konzentration und -Aufnahme

Bemerkenswert war die relativ hohe N-Konzentration der Sprosse in der KontrollVariante, die sich gegenüber den $\mathrm{N}$-Konzentrationen bis $10 \mathrm{ppm} \mathrm{Cd}$ nicht signifikant veränderte (Tab.59). Die N-Aufnahme zeigte einen mehr oder weniger ähnlichen Verlauf wie die TS-Bildung. 
Tab.59: Einfluss der Cd-Behandlung (mg/kg Boden) auf die Konzentration (mg/g TS) und Aufnahme (mg/Topf) von N im Spross von Cupressus sempervirens

\begin{tabular}{|l|r|r|}
\hline Cd mg/kg Boden & $\begin{array}{c}\text { N-Konzentration } \\
\text { (mg/g TS) }\end{array}$ & \multicolumn{2}{|c|}{$\begin{array}{c}\text { N-Aufnahme } \\
\text { (mg/Topf) }\end{array}$} \\
\hline 0,0 & 13,5 & 16,8 \\
1,25 & 13,6 & 15,9 \\
2,5 & 12,0 & 18,6 \\
5 & 11,4 & 12,3 \\
10 & 13,2 & 10,9 \\
20 & 8,1 & 5,2 \\
\hline GD 5\% & 3,6 & 5,8 \\
\hline
\end{tabular}

\section{P-Konzentration und -Aufnahme}

Die P-Konzentration nahm mit steigender Cd-Zufuhr bis 2,5 ppm zu (Tab.60).

Bemerkenswert war die um $43 \%$ deutlich erhöhte P-Konzentration im Spross bei der CdKonzentration von 1,25 mg/kg Boden im Vergleich zur Kontrolle. Danach sank sie, wenn auch nicht signifikant, wieder ab. Die P-Aufnahme verlief in der Tendenz ähnlich wie das STG. 
Tab.60: Einfluss der Cd-Behandlung (mg/kg Boden) auf die Konzentration (mg/g TS) und Aufnahme (mg/Topf) von P, K, Ca und Mg im Spross von Cupressus sempervirens

\begin{tabular}{|l|cccc|cccc|}
\hline \multirow{2}{*}{ mg Cd/kg Boden } & \multicolumn{6}{|c|}{} & \multicolumn{5}{|c|}{ Konzentration $(\mathrm{mg} / \mathrm{g}$ TS) } & \multicolumn{4}{c|}{ Aufnahme (mg/Topf) } \\
\cline { 2 - 9 } & $\mathrm{P}$ & $\mathrm{K}$ & $\mathrm{Ca}$ & $\mathrm{Mg}$ & $\mathrm{P}$ & $\mathrm{K}$ & $\mathrm{Ca}$ & $\mathrm{Mg}$ \\
\hline 0,0 & 1,6 & 14,4 & 5,5 & 1,6 & 2,0 & 17,7 & 6,4 & 1,9 \\
1,25 & 2,3 & 17,4 & 5,3 & 1,7 & 2,6 & 20,5 & 5,9 & 1,8 \\
2,5 & 1,9 & 14,8 & 4,1 & 1,5 & 3,0 & 23,4 & 6,2 & 2,3 \\
5 & 1,6 & 12,4 & 4,2 & 1,5 & 1,7 & 13,5 & 4,5 & 1,7 \\
10 & 1,3 & 9,9 & 5,3 & 1,5 & 1,0 & 8,0 & 4,3 & 1,2 \\
20 & 1,1 & 8,3 & 1,7 & 1,2 & 0,7 & 5,3 & 1,1 & 0,8 \\
\hline \multirow{2}{*}{$\mathrm{GD} \mathrm{5 \%}$} & 0,6 & 2,7 & 2,2 & 0,4 & 0,8 & 7,3 & 1,8 & 0,6 \\
\hline
\end{tabular}

K-Konzentration und -Aufnahme

Die K-Konzentrationen und -Aufnahmen stiegen im Spross von Cupressus-Pflanzen mit den Cd-Stufen bis $2,5 \mathrm{mg} / \mathrm{kg}$ Boden an (Tab.60). Die Cd-Stufe 1,25 mg/kg Boden zeigte gegenüber der Kontrolle eine um $21 \%$ höhere K-Konzentration. Weiter steigende CdGaben über $2,5 \mathrm{mg} / \mathrm{kg}$ Boden hatten eine Verringerung der Konzentration und Aufnahme von K zur Folge. Die höchste K-Aufnahme bei 2,5 mg Cd/kg Boden lag um 32 \% höher als die Kontrolle. Die höchste Cd-Stufe $20 \mathrm{ppm}$ verursachte eine Verringerung der KKonzentration um $42 \%$ gegenüber der Kontrolle. Der niedrigste K-Aufnahme, ein Drittel im Vergleich zur den Kontrollpflanzen, wurde ebenfalls bei der höchsten Cd-Stufe 20 ppm festgestellt.

\section{Ca-Konzentration und -Aufnahme}

Die höchste Cd-Stufe $20 \mathrm{mg} / \mathrm{kg}$ Boden ergab eine Ca-Konzentration im Spross von etwa einem Drittel der Ca-Konzentration in den Kontrollpflanzen (Tab.60). Die Ca-Aufnahme bei dieser Cd-Stufe machte jedoch nur ein Sechstel der Kontrolle aus. Die Ca-Aufnahme verlief in der Tendenz ähnlich wie das STG.

Mg-Konzentration und -Aufnahme

Tab.60 zeigt, dass die niedrigste Mg-Konzentration bei der Cd-Stufe $20 \mathrm{mg} / \mathrm{kg}$ Boden ermittelt wurde. Sie lag um $24 \%$ unter der Kontrolle. Die Mg-Aufnahme verlief im 
wesentlichen entsprechend dem Wachstum und nahm mit steigenden Cd-Stufen geringfügig ab. Bei der Cd-Stufe 2,5 mg/kg Boden stieg die Mg-Aufnahme entsprechend dem Wachstum um $23 \%$ gegenüber der Kontrolle an, jedoch nicht signifikant. Die niedrigste Mg-Aufnahme wurde bei der Cd-Stufe $20 \mathrm{mg} / \mathrm{kg}$ Boden festgestellt. Sie lag um $59 \%$ niedriger als in der Kontrolle.

\section{Cd-Konzentration und -Aufnahme}

Wie beim Spross von A. saligna und $C$. equisetifolia stieg die Cd-Konzentration bzw.Aufnahme auch im Spross von C. sempervirens mit steigenden Cd-Stufen, insbesondere bei den beiden höchsten Cd-Stufen 10 und 20 ppm, gegenüber der Kontrolle drastisch an (Tab.61). Die höchste Cd-Aufnahme wurde bei der Cd-Stufe 10 mg/kg Boden ermittelt. Die beiden höchsten Cd-Stufen beeinträchtigten das Wachstum der Cupressus-Pflanzen stark, stärker als bei den Acacia-Pflanzen.

Tab.61: Einfluss der Cd-Behandlung (mg/kg Boden) auf die Konzentration (mg/g TS) und Aufnahme (mg/Topf) von Cd im Spross von Cupressus sempervirens

\begin{tabular}{|l|r|r|}
\hline mg Cd/kg Boden & $\begin{array}{l}\text { Cd-Konzentration } \\
\text { (mg/g TS) }\end{array}$ & $\begin{array}{l}\text { Cd-Aufnahme } \\
\text { (mg/Topf) }\end{array}$ \\
\hline 0,0 & 0,00 & 0,00 \\
1,25 & 0,05 & 0,06 \\
2,5 & 0,08 & 0,12 \\
5 & 0,17 & 0,18 \\
10 & 0,31 & 0,25 \\
20 & 0,30 & 0,18 \\
\hline GD 5\% & 0,10 & 0,07 \\
\hline
\end{tabular}

\subsection{Cd-Konzentration und -Aufnahme in der Wurzel}

Wie beim Spross stieg sowohl die Cd-Konzentration als auch die Cd-Aufnahme in den Wurzeln mit steigender Cd-Zugabe zum Boden drastisch an (Tab.62). Die Werte beider Parameter lagen in den Wurzeln jedoch viel höher als in den Sprossen. 
Tab.62: Einfluss der Cd-Behandlung (mg/kg Boden) auf die Konzentration (mg/g TS) und Aufnahme (mg/Topf) von Cd in der Wurzel von Cupressus sempervirens

\begin{tabular}{|l|r|r|}
\hline mg Cd/kg Boden & $\begin{array}{l}\text { Cd-Konzentration } \\
\text { (mg/g TS) }\end{array}$ & $\begin{array}{l}\text { Cd-Aufnahme } \\
\text { (mg/Topf) }\end{array}$ \\
\hline 0,0 & 0,00 & 0,00 \\
1,25 & 0,50 & 0,14 \\
2,5 & 0,87 & 0,30 \\
5 & 1,10 & 0,26 \\
10 & 1,35 & 0,19 \\
20 & 2,10 & 0,25 \\
\hline GD 5 \% & 0,35 & 0,12 \\
\hline
\end{tabular}

\subsection{Der pH-Wert des Boden}

Wie bei den anderen beiden Baumarten sank auch der pH-Wert im Boden bei den Cupressus-Pflanzen am Ende des Versuchs von 7 bis auf 4,84 ab (Tab.63). Der pH-Wert wurde jedoch von den Cd-Behandlungen nicht beeinflusst.

Tab. 63: Einfluss der Cd-Behandlung (mg/kg Boden) auf den pH-Wert im Boden von Cupressus sempervirens am Ende des Versuches

\begin{tabular}{|l|r|r|c|c|c|c|l|}
\hline $\mathrm{mg} \mathrm{Cd} / \mathrm{kg}$ Boden & 0,0 & 1,25 & 2,5 & 5 & 10 & 20 & $\mathrm{GD} 5 \%$ \\
\hline $\mathrm{pH}$ & 4,9 & 4,8 & 4,9 & 4,9 & 4,9 & 4,9 & $\mathrm{~ns}$ \\
\hline
\end{tabular}




\subsubsection{Pb-Behandlung}

\subsection{Sprosstrockengewicht (STG)}

Wie bei den anderen beiden Baumarten A. saligna und C. equisetifolia zeigte sich auch bei $C$. sempervirens, dass sich das $\mathrm{STG}$ bei allen $\mathrm{Pb}$-Stufen erhöhte, insbesondere bei 20 ppm Pb (Tab.64). Bei den Acacia-Pflanzen war die Steigerung im STG nur bis zur PbStufe $20 \mathrm{mg} / \mathrm{kg}$ Boden signifikant. Wie bei den Casuarina-Pflanzen, lag das STG der Cupressus-Pflanzen bei der höchsten Pb-Stufe über dem der Kontroll-Pflanzen. Das signifikant höchste STG der Cupressus-Pflanzen wurde sogar bei der Pb-Stufe $20 \mathrm{mg} / \mathrm{kg}$ Boden mit etwa $39 \%$ über dem der Kontrolle festgestellt.

Tab.64: Einfluss der Pb-Behandlung (mg/kg Boden) auf das Sprosstrockengewicht (g/Topf) von Cupressus sempervirens

\begin{tabular}{|l|l|l|l|l|l|l|l|}
\hline mg Pb/kg Boden & 0,0 & 2,5 & 5 & 10 & 20 & 40 & GD 5\% \\
\hline STG g/Topf & 1,17 & 1,22 & 1,27 & 1,29 & 1,62 & 1,40 & 0,29 \\
\hline
\end{tabular}

\subsection{Wurzeltrockengewicht (WTG)}

Das Wurzeltrockengewicht zeigte bei den $\mathrm{Pb}$-Behandlungen eine ähnliche Tendenz wie das STG (Tab. 65). Die höchste TS-Bildung in den Wurzeln war bei der Stufe $20 \mathrm{mg} / \mathrm{kg}$ Boden zu finden und lag um $30 \%$ höher als in der Kontrolle.

Tab.65: Einfluss der Pb-Behandlung (mg/kg Boden) auf das Wurzeltrockengewicht (g/Topf) von Cupressus sempervirens

\begin{tabular}{|l|l|l|l|l|l|l|l|}
\hline mg Pb/kg Boden & 0,0 & 2,5 & 5 & 10 & 20 & 40 & GD 5 \% \\
\hline WTG g/Topf & 0,37 & 0,38 & 0,44 & 0,43 & 0,48 & 0,39 & 0,08 \\
\hline
\end{tabular}


5.1.3.3.3 Konzentration und Aufnahme von $\mathrm{N}, \mathrm{P}, \mathrm{K}, \mathrm{Ca}, \mathrm{Mg}$ und $\mathrm{Pb}$ im Spross

N-Konzentration und -Aufnahme

Die N-Konzentration zeigte keine signifikanten Unterschiede zwischen der Kontrolle und allen $\mathrm{Pb}$-Stufen (Tab.66). Nur die Pb-Stufe 20 ppm führte zu einer signifikanten Steigerung der N-Aufnahme um etwa $49 \%$ über dem der Kontrolle.

Tab.66: Einfluss der Pb-Behandlung ( $\mathrm{mg} / \mathrm{kg}$ Boden) auf die Konzentration ( $\mathrm{mg} / \mathrm{g}$ TS) und Aufnahme (mg/Topf) von N im Spross von Cupressus sempervirens

\begin{tabular}{|l|r|r|}
\hline mg Pb/kg Boden & $\begin{array}{l}\text { N-Konzentration } \\
\text { (mg/g TS) }\end{array}$ & $\begin{array}{l}\text { N-Aufnahme } \\
\text { (mg/Topf) }\end{array}$ \\
\hline 0,0 & 13,6 & 15,8 \\
2,5 & 13,8 & 17,0 \\
5 & 13,2 & 16,6 \\
10 & 13,5 & 17,2 \\
20 & 14,4 & 23,5 \\
40 & 12,0 & 16,8 \\
\hline GD 5\% & ns & 5,1 \\
\hline
\end{tabular}

\section{P-Konzentration und -Aufnahme}

Die P-Konzentration und -Aufnahme verringerte sich im Spross der Cupressus-Pflanzen mit zunehmenden $\mathrm{Pb}$-Gaben (Tab.67). Die höchste $\mathrm{Pb}$-Stufe $40 \mathrm{ppm}$ ergab die niedrigste $\mathrm{P}$ Konzentration bzw. -Aufnahme. Sie lag um 40 \% bzw. $29 \%$ unter der Kontrolle. Die PKonzentrationen bzw. -Aufnahmen bei den restlichen $\mathrm{Pb}$-Stufen unterschieden sich nicht wesentlich von der jeweiligen Kontrolle. 
Tab. 67: Einfluss der Pb-Behandlung (mg/kg Boden) auf die Konzentration (mg/g TS) und Aufnahme (mg/Topf) von P, K, Ca und Mg im Spross von Cupressus sempervirens

\begin{tabular}{|l|llll|lllll|}
\hline \multirow{2}{*}{ mg Pb/kg Boden } & \multicolumn{6}{|l|}{ Konzentration (mg/g TS) } & \multicolumn{4}{|c}{ Aufnahme (mg/Topf) } \\
\cline { 2 - 9 } & $\mathrm{P}$ & $\mathrm{K}$ & $\mathrm{Ca}$ & $\mathrm{Mg}$ & $\mathrm{P}$ & $\mathrm{K}$ & $\mathrm{Ca}$ & $\mathrm{Mg}$ \\
\hline 0,0 & 2,0 & 13,9 & 6,8 & 1,5 & 2,4 & 16,4 & 7,7 & 1,8 \\
2,5 & 1,9 & 15,6 & 6,2 & 1,5 & 2,4 & 18,9 & 7,4 & 1,9 \\
5 & 1,7 & 13,3 & 6,2 & 1,5 & 2,3 & 17,6 & 7,3 & 1,9 \\
10 & 1,6 & 13,2 & 4,8 & 1,5 & 2,0 & 16,9 & 6,1 & 1,9 \\
20 & 2,0 & 14,2 & 5,2 & 1,4 & 3,3 & 23,0 & 8,3 & 2,3 \\
40 & 1,2 & 12,8 & 5,1 & 1,3 & 1,7 & 17,8 & 6,9 & 1,8 \\
\hline \multirow{2}{*}{$\mathrm{GD} \%$} & 0,5 & $\mathrm{~ns}$ & $\mathrm{~ns}$ & $\mathrm{~ns}$ & 0,9 & 5,1 & $\mathrm{~ns}$ & $\mathrm{~ns}$
\end{tabular}

K-Konzentration und -Aufnahme

Die K-Konzentration wurde von der Pb-Behandlung kaum beeinflusst (Tab.67). Mit Ausnahme der K-Aufnahme bei der zweit höchsten Pb-Stufe, wo sie entsprechend dem besten Wachstum um etwa $41 \%$ höher als die Kontrolle lag, unterschieden sich die K-Aufnahmen bei den restlichen $\mathrm{Pb}$-Behandlungen nur wenig von der Kontrolle bzw. voneinander.

Ca-Konzentration und -Aufnahme

Die Ca-Konzentration bzw. -Aufnahme im Spross der Cupressus-Pflanzen nahm mit steigenden $\mathrm{Pb}$-Zugaben leicht ab (Tab. 67). Die höchste $\mathrm{Pb}$-Stufe 40 ppm wies die niedrigsten Werte auf.

Mg-Konzentration und -Aufnahme

Steigende $\mathrm{Pb}$-Stufen führten, allgemein $\mathrm{zu}$ unwesentlichen Unterschieden bei der $\mathrm{Mg}$ Konzentration bzw. -Aufnahme(Tab. 67). Die höchste Mg-Aufnahme wurde entsprechend dem Wachstum bei der Pb-Stufe 20 ppm mit $28 \%$ über der Kontrolle festgestellt.

$\mathrm{Pb}$-Konzentration und -Aufnahme

Die Pb-Konzentration bzw. Pb-Aufnahme im Spross der Cupressus-Pflanzen zeigte im allgemeinen das gleiche Bild wie bei den Casuarina- und Acacia-Pflanzen, im dem sie mit 
steigenden $\mathrm{Pb}$-Stufen stark anstieg (Tab.68). Die höchste Pb-Konzentration bzw. -Aufnahme wurde bei $40 \mathrm{mg} \mathrm{Pb/kg} \mathrm{Boden} \mathrm{bestimmt} \mathrm{und} \mathrm{war} \mathrm{jeweils} \mathrm{etwa} 21 \mathrm{mal}$ so hoch wie in der Kontrolle.

Tab.68: Einfluss der Pb-Behandlung (mg/kg Boden) auf die Konzentration (mg/g TS) und Aufnahme (mg/Topf) von Pb im Spross von Cupressus sempervirens

\begin{tabular}{|l|r|r|}
\hline mg Pb/kg Boden & $\begin{array}{l}\text { Pb-Konzentration } \\
\text { (mg/g TS) }\end{array}$ & $\begin{array}{l}\text { Pb-Aufnahme } \\
\text { (mg/Topf })\end{array}$ \\
\hline 0,0 & 0,003 & 0,004 \\
2,5 & 0,004 & 0,005 \\
5 & 0,008 & 0,010 \\
10 & 0,022 & 0,028 \\
20 & 0,038 & 0,062 \\
40 & 0,062 & 0,086 \\
\hline GD $5 \%$ & 0,008 & 0,000 \\
\hline
\end{tabular}

\subsection{Pb-Konzentration und -Aufnahme in der Wurzel}

Wie im Spross stieg die Pb-Konzentration bzw. -Aufnahme ebenfalls in den Wurzeln der Cupressus-Pflanzen mit zunehmenden $\mathrm{Pb}$-Gaben an, jedoch viel stärker als im Spross (Tab.69). Bei der höchsten Pb-Stufe 40 ppm wurde die Pb-Konzentration auf das 13fache bzw. die Pb-Aufnahme auf das 4fache im Vergleich zum Sprosse erhöht. 
Tab.69: Einfluss der Pb-Behandlung (mg/kg Boden) auf die Konzentration (mg/g TS) und Aufnahme (mg/Topf) von Pb in der Wurzel von Cupressus sempervirens

\begin{tabular}{|l|r|r|}
\hline mg Pb/kg Boden & $\begin{array}{l}\text { Pb-Konzentration } \\
\text { (mg/g TS) }\end{array}$ & $\begin{array}{l}\text { Pb-Aufnahme } \\
\text { (mg/Topf) }\end{array}$ \\
\hline 0,0 & 0,003 & 0,001 \\
2,5 & 0,044 & 0,017 \\
5 & 0,081 & 0,036 \\
10 & 0,158 & 0,067 \\
20 & 0,290 & 0,133 \\
40 & 0,789 & 0,309 \\
\hline GD 5\% & 0,129 & 0,067 \\
\hline
\end{tabular}

\subsection{Der pH-Wert des Bodens}

Wie in den Zn-und Cd-Varianten sank der Ausgangs-pH-Wert des Bodens am Ende des Versuches durch die Pb-Behandlungen ebenfalls ab, und zwar von 7 bis auf 4,7; die Unterschiede waren jedoch allgemein zwischen den Behandlungen nicht signifikant zur Kontrolle (Tab.70).

Tab. 70: Einfluss der Pb-Behandlung (mg/kg Boden) auf den $\mathrm{pH}-$ Wert im Boden von Cupressus sempervirens am Ende des Versuches

\begin{tabular}{|l|c|c|c|c|c|c|l|}
\hline $\mathrm{mg} \mathrm{Pb} / \mathrm{kg}$ Boden & 0,0 & 2,5 & 5 & 10 & 20 & 40 & GD 5\% \\
\hline $\mathrm{pH}$ & 4,8 & 4,9 & 4,9 & 4,7 & 4,8 & 4,7 & Ns \\
\hline
\end{tabular}




\section{Zusammenfassender Überblick über die Ergebnisse von Versuch 1}

In diesem Versuches sollte die Wirkung verschiedener Konzentrationen der Schwermetalle $\mathrm{Zn}, \mathrm{Cd}$ und $\mathrm{Pb}$ auf Wachstum und Nährstoff-Aufnahme von Acacia saligna, Casuarina equisetifolia und Cupressus sempervirens ermittelt werden. Außer den Schwermetallen wurden die Pflanzen in diesem Versuch normal gedüngt.

Tab. 71 zeigt die Schwermetall-Zugaben zum Boden, bei denen die höchsten Werte an STG, WTG und Nährstoffaufnahmen erhalten wurden.

Tab.71: Schwermetallapplikationen die zu den höchsten Spross-, Wurzel-Massen und Nährstoffaufnahmen im Spross führten (Optimale Wachstumsbedingungen)

\begin{tabular}{|c|c|c|c|c|c|c|c|c|}
\hline \multirow[t]{3}{*}{ Baum } & SM & \multicolumn{7}{|c|}{ höchste } \\
\hline & & $\begin{array}{l}\text { Spoßmasse } \\
\text { (g/Topf) }\end{array}$ & $\begin{array}{c}\text { Wurzelmasse } \\
\text { (g/Topf) }\end{array}$ & $\begin{array}{l}\mathrm{N}- \\
\text { Aufnahme } \\
\text { (mg/Topf) }\end{array}$ & $\begin{array}{l}\text { P- } \\
\text { Aufnahme } \\
\text { (mg/Topf) }\end{array}$ & $\begin{array}{l}\text { K- } \\
\text { Aufnahme } \\
\text { (mg/Topf) }\end{array}$ & $\begin{array}{l}\text { Ca- } \\
\text { Aufnahme } \\
\text { (mg/Topf) }\end{array}$ & $\begin{array}{l}\text { Mg- } \\
\text { Aufnahme } \\
\text { (mg/Topf) }\end{array}$ \\
\hline & & \multicolumn{7}{|c|}{ bei Schwermetall - Zugabe (mg/kg Boden) } \\
\hline Acacia & $\begin{array}{l}\mathrm{Zn} \\
\mathrm{Cd} \\
\mathrm{Pb}\end{array}$ & $\begin{array}{l}10 \\
2,5-5 \\
5-10\end{array}$ & $\begin{array}{l}10 \\
5 \\
10\end{array}$ & $\begin{array}{l}10 \\
5 \\
10\end{array}$ & $\begin{array}{l}0,5-10 \\
5 \\
2,5-5\end{array}$ & $\begin{array}{l}0,5-10 \\
5 \\
0-2,5\end{array}$ & $\begin{array}{l}10 \\
5 \\
20\end{array}$ & $\begin{array}{l}2,5-10 \\
1,25 \\
0 \quad-5\end{array}$ \\
\hline Casuarina & $\begin{array}{l}\mathrm{Zn} \\
\mathrm{Cd} \\
\mathrm{Pb}\end{array}$ & $\begin{array}{l}10-20 \\
2,5 \\
5-10\end{array}$ & $\begin{array}{ll}0 & -20 \\
1,25 & -10 \\
2,5 & \end{array}$ & $\begin{array}{ll}0 & -10 \\
0 & -2,5 \\
2,5 & -10\end{array}$ & $\begin{array}{l}0-20 \\
2,5 \\
20\end{array}$ & $\begin{array}{l}10 \\
0 \\
2,5-10\end{array}$ & $\begin{array}{l}10-20 \\
1,25 \\
5-10\end{array}$ & $\begin{array}{l}0 \quad-20 \\
1,25-2,5 \\
20\end{array}$ \\
\hline Cupressus & $\begin{array}{l}\mathrm{Zn} \\
\mathrm{Cd} \\
\mathrm{Pb}\end{array}$ & $\begin{array}{l}10 \\
2,5 \\
20\end{array}$ & $\begin{array}{l}2,5-10 \\
2,5 \\
20\end{array}$ & $\begin{array}{l}10 \\
2,5 \\
20\end{array}$ & $\begin{array}{l}10 \\
1,25-2,5 \\
20\end{array}$ & $\begin{array}{l}2,5-10 \\
2,5 \\
20\end{array}$ & $\begin{array}{ll}0 & -2,5 \\
0 & -2,5 \\
20\end{array}$ & $\begin{array}{l}0,5-2,5 \\
2,5 \\
20\end{array}$ \\
\hline
\end{tabular}

$\mathrm{SM}=$ Schwermetalle

Bei Applikation von Zn wiesen die Acacia- und Cupressus-Pflanzen die höchste TS-Bildung in ihren Sprossen bei Zn-Zugabe von $10 \mathrm{mg} / \mathrm{kg}$ Boden auf. Die Casuarina-Pflanzen zeigten eine höhere Toleranz gegenüber höchsten Zn-Zugaben und produzierten das höchste STG sogar bis zur Zn-Zugabe von 20 ppm. Die TS-Bildung in den Wurzeln von A. saligna 
entsprach der Spross-Entwicklung, wenn Zn verabreicht wurde. Die Casuarina-Pflanzen bildeten sogar ein starkes Wurzelsystem bei der zuletzt genannten $\mathrm{Zn}$-Behandlung. Die Wurzeln der Cupressus-Pflanzen entwickelten sich optimal bei Zn-Zufuhr von 2,5-10 mg/kg Boden.

Bei Zn-Zufuhr nahmen die in diesem Versuch normal gedüngten Pflanzen der drei Baumarten die höchste N-Menge bei der Zn-Behandlung von 10 ppm auf. Die höchste P-Aufnahme der drei Baumarten variierte je nach Zn-Behandlung. Während die höchste P-Aufnahme der Acacia-Pflanzen bei Zugabe von 0,5 bis $10 \mathrm{ppm} \mathrm{Zn}$ ermittelt wurde, nahmen die CasuarinaPflanzen höchste P-Mengen bei 0 bis 20 ppm Zn auf. Der höchste P-Aufnahme der Cupressus-Pflanzen lag bei der Zn-Stufe $10 \mathrm{mg} / \mathrm{kg}$ Boden. Die K-Aufnahme von A. saligna und C. sempervirens zeigte keine starke Abhängigkeit von der Zn-Behandlung, denn hohe Werte wurden in einem relativ breiten Bereich von Zn-Behandlungen gefunden, während die Casuarina-Pflanzen die höchste K-Aufnahme bei der Stufe $10 \mathrm{mg} \mathrm{Zn/kg} \mathrm{Boden} \mathrm{aufwiesen.}$ Die höchsten Ca-Aufnahmen von den Acacia- und Casuarina-Pflanzen entsprechend dem STG bzw. WTG. Anders war es bei C. sempervirens. Die höchste Mg-Aufnahme trat hier bei ähnlichen Zn-Zugaben ein, wie die höchste Ca-Aufnahme.

Die Acacia-Pflanzen tolerierten Cd-Zugaben zum Boden bis $5 \mathrm{mg} / \mathrm{kg}$ Boden, wo sie das höchste STG produzierten. Die anderen beiden Baumarten ergaben das höchste STG bei Zugabe von 2,5 ppm Cd.

Bei der Bildung der höchsten Wurzel-TS unterschieden sich die drei Baumarten deutlich voneinander. Die Casuarina-Pflanzen waren toleranter und bildeten die höchsten WTG bis zur Cd-Zugabe von 10 ppm. Weniger tolerant in dieser Hinsicht waren die Acacia-Pflanzen, die das höchste WTG bei der Cd-Stufe 5 ppm ergaben. Bei den Cupressus-Pflanzen war dies bei 2,5 ppm Cd der Fall.

Die höchste $\mathrm{N}-$ und P-Aufnahme entsprachen bei den drei Baumarten dem STG. Die höchste K-Aufnahme der Casuarina-Pflanzen wurde ohne Cd-Zugabe festgestellt. Bei den anderen beiden Baumarten entsprachen sie dem STG. Ähnlich waren bei den drei Baumarten die höchste Ca-Aufnahme. Die höchsten Mg-Aufnahmen der Casuarina- und Cupressus-Pflanzen wurden bei den höchsten STG ermittelt.

Bei der Applikation des $\mathrm{Pb}$ wiesen die Acacia- und Cupressus-Pflanzen höhere Toleranz gegenüber diesem Schwermetall auf und lieferten die höchsten STG bis zur Pb-Gabe von 20 mg/kg Boden. Die höchste Toleranz der Casuarina-Pflanzen lag bei 10 mg/kg Boden. 
Das höchste WTG unter der Wirkung der Pb-Zufuhr korrelierte mit dem höchsten STG bei den Acacia- und Cupressus-Pflanzen. Bei den Casuarina-Pflanzen wurde das höchste WTG bei einer niedrigeren $\mathrm{Pb}$-Stufe, nämlich bei $2,5 \mathrm{mg} / \mathrm{kg}$ Boden, festgestellt.

Die höchste N-Aufnahme entsprach der höchsten TS-Bildung bei den drei Baumarten. Die höchste Aufnahme von $\mathrm{P}, \mathrm{K}, \mathrm{Ca}$ und $\mathrm{Mg}$ folgte meistens der höchsten TS-Bildung unter Einfluss der Pb-Applikation.

Im allgemeinen stieg die Konzentration und Aufnahme von $\mathrm{Zn}, \mathrm{Cd}$ und $\mathrm{Pb}$ sowohl in den Sprossen als auch in den Wurzeln der drei Baumarten mit zunehmender Zugabe der drei Schwermetalle zum Boden stark an.

Tab. 72 stellt das Verhältnis der Werte bei den höchsten bzw. toxischen Schwermetall-Gaben in $\%$ zu den höchsten Werten (optimal $=100$ ) bei verschiedenen Schwermetall-Gaben dar. Die unterstrichenen Werte zeigen die höchste Toleranz der Pflanzen der drei Baumarten in Form der höchsten TS-Bildung im Spross und in der Wurzel, sowie die Fähigkeit der Pflanzen, Nährstoffe aufzunehmen bei den höchsten Schwermetall-Gaben in \% im Vergleich zu den optimalen (höchsten) Werten.

Aus dieser Betrachtungsweise war die toxische Wirkung von $\mathrm{Zn}$ bei A. saligna und C. equisetifolia sehr ähnlich hinsichtlich der Nährstoffaufnahme und WTG, nur das Wachstum der Sprosse und N-Aufnahme wurden bei C. equisetifolia weniger geschädigt als bei A. saligna. Bei C. sempervirens ergaben sich beim WTG und den Aufnahme von $\mathrm{P}$ und $\mathrm{K}$ größere Abnahmen als bei den anderen Pflanzen.

Alle Pflanzenarten wurden durch Cd stärker geschädigt als durch Zn. Am geringsten waren die Abnahmen des STG und des WTG bei C. sempervirens, während die Nährstoffaufnahmen bei $C$. equisetifolia und $C$. sempervirens meist geringer waren als bei A. saligna. Nur die Mg-Aufnahme wurde bei $C$. sempervirens weniger gestört als bei den anderen Baumarten.

$\mathrm{Pb}$ schädigte die Pflanzen weniger als die anderen Schwermetalle. Unterschiede zwischen den Pflanzen zeigte vor allem die P-Aufnahme, die bei A. saligna kaum beeinträchtigt wurde, bei C. sempervirens aber sehr stark, während die Mg-Aufnahme bei A. saligna stark eingeschränkt war, deutlich weniger aber bei den anderen Arten. 
Tab. 72: Das Verhältnis der Werte der höchsten bzw. toxischen SchwermetallGaben in \% zu den höchsten Werten (optimal =100) bei verschiedenen Schwermetall-Gaben

\begin{tabular}{|c|c|c|c|c|c|c|c|c|c|}
\hline Baum & SM & $\begin{array}{l}\text { HSMG } \\
\mathrm{mg} / \mathrm{kg} \\
\text { Boden }\end{array}$ & $\begin{array}{l}\text { STG } \\
\\
\\
(\%)\end{array}$ & $\begin{array}{l}\text { WTG } \\
(\%)\end{array}$ & $\begin{array}{l}\mathrm{N}- \\
\text { Aufnahme } \\
(\%)\end{array}$ & $\begin{array}{l}\text { P- } \\
\text { Aufnahme } \\
\quad(\%)\end{array}$ & $\begin{array}{l}\text { K- } \\
\text { Aufnahme } \\
(\%)\end{array}$ & $\begin{array}{l}\text { Ca- } \\
\text { Aufnahme } \\
\quad(\%)\end{array}$ & $\begin{array}{l}\mathrm{Mg}- \\
\text { Aufnahme } \\
(\%)\end{array}$ \\
\hline & & & \multicolumn{7}{|c|}{ Die Werte bei HSMG in \% von den Optimalen Werten bei SMG } \\
\hline Acacia & $\begin{array}{l}\mathrm{Zn} \\
\mathrm{Cd} \\
\mathrm{Pb}\end{array}$ & $\begin{array}{l}40 \\
20 \\
40\end{array}$ & $\begin{array}{l}58 \% \\
33 \%-* \\
71 \%\end{array}$ & $\begin{array}{l}\frac{56 \%}{10 \%} \\
72 \%\end{array}$ & $\begin{array}{l}48 \% \\
43 \%-* \\
73 \%-*\end{array}$ & $\begin{array}{l}63 \% \\
28 \%-* \\
94 \%\end{array}$ & $\begin{array}{l}64 \% \\
40 \%-* \\
82 \%-*\end{array}$ & $\begin{array}{l}54 \% \\
32 \%-* \\
75 \%\end{array}$ & 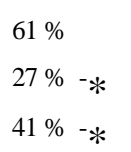 \\
\hline Casuarina & $\begin{array}{l}\mathrm{Zn} \\
\mathrm{Cd} \\
\mathrm{Pb}\end{array}$ & $\begin{array}{l}40 \\
20 \\
40\end{array}$ & $\begin{array}{l}\underline{68 \%} \\
25 \%-* \\
78 \%+*\end{array}$ & $\begin{array}{l}\underline{56 \%} \\
19 \%-* \\
70 \%\end{array}$ & $\begin{array}{l}65 \% \\
66 \% \\
24 \%-* \\
75 \%\end{array}$ & $\begin{array}{l}66 \%-* \\
71 \% \\
30 \%-* \\
69 \%\end{array}$ & $\begin{array}{l}55 \% \\
20 \%-* \\
76 \%\end{array}$ & $\begin{array}{l}69 \% \\
19 \%-* \\
82 \%+*\end{array}$ & $\begin{array}{l}61 \% \\
17 \%-* \\
86 \%+*\end{array}$ \\
\hline Cupressus & $\begin{array}{l}\mathrm{Zn} \\
\mathrm{Cd}\end{array}$ & $\begin{array}{l}40 \\
20\end{array}$ & $\begin{array}{l}58 \%-* \\
42 \%-* \\
86 \%\end{array}$ & $\begin{array}{l}46 \%-* \\
36 \%-* \\
\underline{81 \%}\end{array}$ & $\begin{array}{l}43 \% \\
28 \%-* \\
71 \%\end{array}$ & $\begin{array}{l}32 \%-* \\
24 \%-* \\
51 \%\end{array}$ & $\begin{array}{l}36 \%-* \\
23 \%-* \\
77 \%\end{array}$ & $\begin{array}{l}59 \% \\
17 \%-* \\
18 \%{ }^{-*} \\
83 \%\end{array}$ & $\begin{array}{l}54 \% \\
35 \%-* \\
78 \%\end{array}$ \\
\hline
\end{tabular}

$\mathrm{SM}=$ Schwermetalle $\quad \mathrm{SMG}=$ Schwermetall - Gaben $\quad$ HSMG $=$ Höchste Schwermetall - Gaben

${ }^{*}=$ signifikant niedriger als Kontrolle, also " toxischer " Effekt $\quad+_{*}=$ signifikant höher als Kontrolle

Der Ausgangs-pH des Bodens sank bei den Schwermetall-Behandlungen bis zum Ende des Versuches allgemein ab. Die Werte unterschieden sich meistens von der Kontrolle bzw. voneinander nicht signifikant. 


\section{2 Versuch 2}

Im ersten Versuch wurde die Wirkung von $\mathrm{Pb}$ auf Wachstum und Nährstoffaufnahme der drei Baumarten bei Anwendung des stickstoffhaltigen Bleinitrats untersucht. Das Ziel des zweiten Versuches war, die Wirkung dieses Schwermetalls $(\mathrm{Pb})$ ebenfalls bei Anwendung von Bleinitrat, jedoch unter Berücksichtigung des in diesem Salz enthaltenen Stickstoffs zu prüfen, um eine Nebenwirkung dieses begleitenden $\mathrm{N}$ auszuschließen. Das Blei wurde in sechs Stufen mit vier Wiederholungen je Stufe getestet. Bei jeder Pb-Stufe wurde die NMenge des $\mathrm{Pb}\left(\mathrm{NO}_{3}\right)_{2}$ von der $\mathrm{N}$-Düngungsmenge abgezogen. Bei der höchsten $\mathrm{Pb}$-Stufe $40 \mathrm{mg} / \mathrm{kg}$ Böden betrug diese Menge 5,41 mg N/kg Boden. Die drei Baumarten des 1. Versuches dienten ebenfalls im 2. Versuch als Versuchspflanzen.

\subsubsection{Acacia saligna}

\subsubsection{Sprosstrockengewicht (STG)}

Das Sprosstrockengewicht zeigte eine signifikante Zunahme bei allen $\mathrm{Pb}$-Stufen gegenüber der Kontrolle (Tab.73). Besonders deutlich war dies bei der Pb-Stufe 10 ppm, wo das STG 2,4-mal höher lag als in der Kontrolle. Bei der höchsten Pb-Stufe 40 ppm nahm das STG zwar wieder ab, lag jedoch um $66 \%$ über der Kontrolle.

Tab.73: Einfluss der $\mathrm{Pb}$-Behandlung (mg/kg Boden) als $\mathrm{Pb}\left(\mathrm{NO}_{3}\right)_{2}$ auf das Sprosstrockengewicht (g/Topf) von Acacia saligna

\begin{tabular}{|l|r|r|c|c|c|c|l|}
\hline mg Pb/kg Boden & 0,0 & 2,5 & 5 & 10 & 20 & 40 & GD 5\% \\
\hline STG g/Topf & 3,5 & 6,2 & 7,7 & 8,5 & 7,4 & 5,8 & 2,0 \\
\hline
\end{tabular}

\subsubsection{Wurzeltrockengewicht (WTG)}

Das Wurzeltrockengewicht zeigte eine ähnliche Tendenz wie das STG (Tab.74), aber ohne signifikante Steigerung bei der letzten $\mathrm{Pb}$-Stufe $40 \mathrm{ppm}$. Anders als beim Spross, lag das optimale WTG bei der Pb-Stufe 5 ppm höher als in der Kontrolle. 
Tab.74: Einfluss der $\mathrm{Pb}$-Behandlung (mg/kg Boden) als $\mathrm{Pb}\left(\mathrm{NO}_{3}\right)_{2}$ auf das Wurzeltrockengewicht (g/Topf) von Acacia saligna

\begin{tabular}{|l|l|l|c|c|c|c|l|}
\hline $\mathrm{mg} \mathrm{Pb} / \mathrm{kg}$ Boden & 0,0 & \multicolumn{1}{|c|}{2,5} & 5 & 10 & 20 & 40 & GD 5\% \\
\hline WTG g/Topf & 1,3 & 2,4 & 4,3 & 3,7 & 3,1 & 1,5 & 0,8 \\
\hline
\end{tabular}

5.2.1.3. Konzentration und Aufnahme von $\mathrm{N}, \mathrm{P}, \mathrm{K}, \mathrm{Ca}, \mathrm{Mg}$ und $\mathrm{Pb}$ im Spross N-Konzentration und -Aufnahme

Steigende $\mathrm{Pb}-$ Gaben führten $\mathrm{zu}$ einer Abnahme der N-Konzentration, die niedrigste $\mathrm{N}$ Konzentration mit nur $47 \%$ des Kontrollwertes, war bei der höchsten Pb-Stufe 40 mg/kg Boden festzustellen (Tab.75). Die N-Aufnahme entsprach dem Wachstum. Sie zeigte eine Zunahme mit steigender Pb-Zugabe bis zu 20 ppm, wobei das höchste STG die höchste NAufnahme mit $74 \%$ über der Kontrolle aufwies. Die höchste Pb-Zugabe verringerte die NAufnahme um nur $18 \%$ nicht signifikant gegenüber der Kontrolle.

Tab.75: Einfluss der $\mathrm{Pb}-$ Behandlung (mg/kg Boden) als $\mathrm{Pb}\left(\mathrm{NO}_{3}\right)_{2}$ auf die Konzentration (mg/g TS) und Aufnahme (mg/Topf) von N im Spross Acacia saligna

\begin{tabular}{|l|r|r|}
\hline mg Pb/kg Boden & $\begin{array}{c}\text { N-Konzentration } \\
\text { (mg/g TS) }\end{array}$ & $\begin{array}{l}\text { N-Aufnahme } \\
\text { (mg/Topf) }\end{array}$ \\
\hline 0,0 & 20,2 & 65,9 \\
2,5 & 16,5 & 102,3 \\
5 & 14,3 & 109,8 \\
10 & 13,3 & 114,9 \\
20 & 12,3 & 88,7 \\
40 & 9,5 & 54,3 \\
\hline GD 5\% & 5,5 & 44,8 \\
\hline
\end{tabular}

\section{P-Konzentration und -Aufnahme}

Zwischen den $\mathrm{Pb}$-Stufen und der Kontrolle ergaben sich keine signifikanten Unterschiede der P-Konzentration (Tab.76). Bei der höchsten Pb-Zugabe $40 \mathrm{mg} / \mathrm{kg}$ Boden sank die PKonzentration auf weniger als die Hälfte der Kontrolle ab. Die P-Aufnahme entsprach 
allgemein dem Wachstum. Bei der Pb-Stufe 5 ppm stieg sie auf das 2,3fache im Vergleich zur Kontrolle. Signifikante Steigerungen der P-Aufnahme wurden bei 2,5; 5; 10 und 20 ppm $\mathrm{Pb}$ gegenüber der Kontrolle festgestellt. Bei der höchsten Pb-Gabe erhöhte sich die PAufnahme um $43 \%$ im Vergleich zur Kontrolle, gegenüber einer Steigerung des STG um $67 \%$.

Tab.76: Einfluss der Pb-Behandlung (mg/kg Boden) als $\mathrm{Pb}\left(\mathrm{NO}_{3}\right)_{2}$ auf die Konzentration (mg/g TS) und Aufnahme (mg/Topf) von P, K, Ca und Mg im Spross von Acacia saligna

\begin{tabular}{|l|cccc|cccc|}
\hline \multirow{2}{*}{ mg Pb/kg Boden } & \multicolumn{7}{|l|}{ Konzentration (mg/g TS) } & \multicolumn{4}{ll}{ Aufnahme (mg/Topf) } \\
\cline { 2 - 8 } & $\mathrm{P}$ & $\mathrm{K}$ & $\mathrm{Ca}$ & $\mathrm{Mg}$ & $\mathrm{P}$ & $\mathrm{K}$ & $\mathrm{Ca}$ & $\mathrm{Mg}$ \\
\hline \multirow{2}{*}{0,0} & 2,8 & 11,3 & 11,6 & 2,9 & 9,4 & 36,1 & 39,8 & 9,7 \\
2,5 & 2,4 & 9,6 & 13,7 & 3,2 & 15,1 & 58,9 & 85,8 & 19,6 \\
5 & 3,0 & 6,5 & 14,0 & 3,2 & 21,8 & 49,1 & 103,8 & 23,5 \\
10 & 2,2 & 6,7 & 13,0 & 2,6 & 18,3 & 55,6 & 107,7 & 22,3 \\
20 & 2,8 & 7,8 & 13,1 & 2,4 & 20,2 & 54,9 & 95,7 & 17,5 \\
40 & 2,3 & 8,8 & 11,5 & 2,5 & 13,4 & 50,2 & 66,4 & 14,4 \\
\hline \multirow{2}{*}{ GD 5\% } & $\mathrm{ns}$ & 3,0 & $\mathrm{~ns}$ & $\mathrm{~ns}$ & 5,6 & 11,3 & 29,6 & 5,7 \\
\hline
\end{tabular}

K-Konzentration und -Aufnahme

Die K-Konzentration im Spross der Acacia-Pflanzen zeigte eine deutliche Verringerung mit zunehmender Pb-Zugabe (Tab.76). Signifikante Verringerungen der K-Konzentration wurden bei Pb-Gaben von 5, 10 und 20 ppm gegenüber der Kontrolle ermittelt. Bei der höchsten $\mathrm{Pb}$-Zugabe 40 ppm nahm die K-Konzentration gegenüber der Kontrolle um $22 \%$ ab. Die K-Aufnahme war in allen $\mathrm{Pb}-$ Stufen wegen des verbesserten Wachstums signifikant höher als in der Kontrolle. Bei der niedrigsten Pb-Stufe 2,5 mg/kg Boden wurde die höchste K-Aufnahme festgestellt, die um 63 \% höher als in der Kontrolle lag.

Ca-Konzentration und -Aufnahme

Mit Ausnahme der höchsten $\mathrm{Pb}$-Stufe, wurde die Ca-Konzentration gegenüber der Kontrolle nur geringfügig erhöht (Tab.76). Die höchste Ca-Konzentration im Spross wurde bei der Pb-Stufe 5 ppm mit um $20 \%$ höher als in der Kontrolle gefunden. Die CaAufnahme entsprach im wesentlichen dem Wachstum. Sie stieg gegenüber der Kontrolle bei allen $\mathrm{Pb}$-Stufen an. Signifikante Steigerungen der $\mathrm{Ca}$-Aufnahme wurden bei $\mathrm{Pb}$-Gaben 
2,5; 5; 10 und 20 ppm gegenüber der Kontrolle ermittelt. Die höchste Ca-Aufnahme wurde bei der $\mathrm{Pb}$-Stufe $10 \mathrm{mg} / \mathrm{kg}$ Boden festgestellt und lag etwa 3-mal höher als in der Kontrolle.

Mg-Konzentration und -Aufnahme

Im Spross zeigte sich bei den $\mathrm{Pb}$-Stufen bis zu $5 \mathrm{mg} / \mathrm{kg}$ Boden eine Erhöhung der $\mathrm{Mg}$ Konzentration (Tab.76). Danach nahm die Mg-Konzentration bei den restlichen drei $\mathrm{Pb}$ Gaben 10, 20 und 40 ppm ab. Die höchste Pb-Stufe 40 mg/kg Boden hatte eine um $14 \%$ geringere Mg-Konzentration als die Kontrolle. Steigende $\mathrm{Pb}$-Konzentrationen im Boden führten $\mathrm{zu}$ höheren $\mathrm{Mg}$-Aufnahmen im Spross, was dem Wachstum entsprach. Nach Zufuhr von $5 \mathrm{mg} / \mathrm{kg}$ Boden war die Mg-Aufnahme 2,4fach höher als in der Kontrolle. Bei der höchsten angewandten Menge von $40 \mathrm{ppm} \mathrm{Pb}$ wurde die Mg-Aufnahme um $48 \%$ gegenüber der Kontrolle, jedoch nicht signifikant, erhöht.

$\mathrm{Pb}$-Konzentration und Aufnahme

Erwartungsgemäß akkumulierten die Pflanzen große Pb-Mengen im Spross mit steigenden $\mathrm{Pb}-$ Konzentrationen im Boden (Tab.77). Bei Pb-Zugabe von 40 ppm stieg die $\mathrm{Pb}$ Konzentration auf das 26 fache der Kontrolle. Aufgrund der relativ hohen TG und PbKonzentrationen lag die $\mathrm{Pb}$-Aufnahme im Spross allgemein mehrfach höher als in der Kontrolle. Zum Beispiel wurde die Pb-Aufnahme im Spross der höchsten Pb-Stufe auf das 39 fache der Kontrolle gesteigert.

Tab.77: Einfluss der Pb-Behandlung (mg/kg Boden) als $\mathrm{Pb}\left(\mathrm{NO}_{3}\right)_{2}$ auf die Konzentration (mg/g TS) und Aufnahme (mg/Topf) von Pb im Spross von Acacia saligna

\begin{tabular}{|l|r|r|}
\hline mg Pb/kg Boden & $\begin{array}{c}\text { Pb-Konzentration } \\
\text { (mg/g TS })\end{array}$ & $\begin{array}{l}\text { Pb-Aufnahme } \\
(\mathrm{mg} / \text { Topf })\end{array}$ \\
\hline 0,0 & 0,005 & 0,019 \\
2,5 & 0,017 & 0,103 \\
5 & 0,016 & 0,123 \\
10 & 0,030 & 0,245 \\
20 & 0,064 & 0,455 \\
40 & 0,128 & 0,741 \\
\hline GD $5 \%$ & 0,016 & 0,107 \\
\hline
\end{tabular}




\subsubsection{Pb-Konzentration und -Aufnahme in der Wurzel}

Die Wurzeln wiesen bei ähnlicher Tendenz höhere Pb-Konzentrationen als die Sprosse auf (Tab.78). Die Steigerung der Pb-Applikation führte zu drastischen Erhöhungen der $\mathrm{Pb}$ Konzentration in den Wurzeln. Sie lag bei der höchsten Pb-Stufe 137-mal höher als in der Kontrolle. Wegen der relativ hohen WTG und $\mathrm{Pb}$-Konzentrationen wurde die $\mathrm{Pb}$ Aufnahme in den Wurzeln bei allen Pb-Stufen im Vergleich zur Kontrolle deutlich erhöht. So lag die Pb-Aufnahme bei der höchsten Pb-Stufe 40 ppm 144fach höher als in der Kontrolle.

Tab.78: Einfluss der $\mathrm{Pb}$-Behandlung (mg/kg Boden) als $\mathrm{Pb}\left(\mathrm{NO}_{3}\right)_{2}$ auf die Konzentration (mg/g TS) und Aufnahme (mg/Topf) von $\mathrm{Pb}$ in der Wurzel von Acacia saligna

\begin{tabular}{|l|r|r|}
\hline mg Pb/kg Boden & $\begin{array}{l}\text { Pb-Konzentration } \\
\text { (mg/g TS) }\end{array}$ & $\begin{array}{l}\text { Pb-Aufnahme } \\
\text { (mg/Topf) }\end{array}$ \\
\hline 0,0 & 0,005 & 0,007 \\
2,5 & 0,082 & 0,204 \\
5 & 0,155 & 0,660 \\
10 & 0,251 & 0,921 \\
20 & 0,423 & 1,288 \\
40 & 0,686 & 1,005 \\
\hline GD 5\% & 0,150 & 0,372 \\
\hline
\end{tabular}

\subsubsection{Der pH-Wert des Bodens}

Der $\mathrm{pH}$-Wert des Bodens zeigte bei der $\mathrm{Pb}$-Anwendung einen ähnlichen Verlauf wie im ersten Versuch bei A. saligna, nur bewegte er sich auf einem niedrigeren Niveau (Tab.79). Bei allen Pb-Stufen konnten keine wesentlichen Unterschiede festgestellt werden. Der Ausgangs-pH-Wert sank bis auf 4,6 am Ende des Versuches.

Tab.79: Einfluss der Pb-Behandlung (mg/kg Boden) als $\mathrm{Pb}\left(\mathrm{NO}_{3}\right)_{2}$ auf den pH-Wert im Boden von Acacia saligna am Ende des Versuches

\begin{tabular}{|l|c|c|c|c|c|l|l|}
\hline $\mathrm{mg} \mathrm{Pb} / \mathrm{kg}$ Boden & 0,0 & 2,5 & 5 & 10 & 20 & 40 & GD 5\% \\
\hline $\mathrm{pH}$ & 4,7 & 4,7 & 4,6 & 4,6 & 4,8 & 4,8 & $\mathrm{~ns}$ \\
\hline
\end{tabular}




\subsubsection{Casuarina equisetifolia}

\subsubsection{Sprosstrockengewicht (STG)}

Das Sprosstrockengewicht von C. equisetifolia stieg im allgemeinen mit steigender $\mathrm{Pb}$ Konzentration im Boden an (Tab.80). Signifikante Steigerungen des STG wurden bei den $\mathrm{Pb}$-Stufen 5 und 10 ppm gegenüber der Kontrolle ermittelt. Bei der Pb-Stufe 10 ppm lag das Sprosstrockengewicht um $31 \%$ höher als bei der Kontrolle. Die höchste Pb-Stufe $40 \mathrm{mg} / \mathrm{kg}$ Boden wies aber gegenüber der Kontrolle nur ein geringfügig erhöhtes STG auf.

Tab.80: Einfluss der $\mathrm{Pb}$-Behandlung (mg/kg Boden) als $\mathrm{Pb}\left(\mathrm{NO}_{3}\right)_{2}$ auf das Sprosstrockengewicht (g/Topf) von Casuarina equisetifolia

\begin{tabular}{|l|l|l|l|l|l|l|l|}
\hline $\mathrm{mg} \mathrm{Pb} / \mathrm{kg}$ Boden & 0,0 & 2,5 & 5 & 10 & 20 & 40 & GD 5 \% \\
\hline STG g/Topf & 7,1 & 7,5 & 8,3 & 9,3 & 7,8 & 7,7 & 1,0 \\
\hline
\end{tabular}

\subsubsection{Wurzeltrockengewicht (WTG)}

Die TS-Bildung in den Wurzeln zeigte einen ähnlichen Verlauf wie in den Sprossen (Tab.81). Das höchste WTG wurde bei der Pb-Stufe $5 \mathrm{mg} / \mathrm{kg}$ Boden ermittelt, es lag um $59 \%$ höher als bei der Kontrolle. Signifikante Steigerungen wurden bei 2,5; 5; 10 und $20 \mathrm{mg} \mathrm{Pb} / \mathrm{kg}$ Boden gegenüber der Kontrolle festgestellt.

Tab.81: Einfluss der $\mathrm{Pb}$-Behandlung (mg/kg Boden) als $\mathrm{Pb}\left(\mathrm{NO}_{3}\right)_{2}$ auf das Wurzeltrockengewicht (g/Topf) von Casuarina equisetifolia

\begin{tabular}{|l|r|c|c|c|c|c|l|}
\hline mg Pb/kg Boden & 0,0 & 2,5 & 5 & 10 & 20 & 40 & GD 5 \\
\hline WTG g/Topf & 1,7 & 2,6 & 2,7 & 2,6 & 2,5 & 2,4 & 0,8 \\
\hline
\end{tabular}

\subsubsection{Konzentration und Aufnahme von $\mathrm{N}, \mathrm{P}, \mathrm{K}, \mathrm{Ca}, \mathrm{Mg}$ und $\mathrm{Pb}$ im Spross}

N-Konzentration und -Aufnahme

Die N-Konzentration nahm im Spross von C. equisetifolia mit zunehmender Pb-Zugabe zum Boden meistens leicht ab (Tab.82). Nur bei der Pb-Stufe $5 \mathrm{mg} / \mathrm{kg}$ Boden wurde die NKonzentration im Vergleich zur Kontrolle um $21 \%$ gesteigert. Die N-Aufnahme lag entsprechend dem Wachstum bei Zugabe von 5 bzw. 10 ppm Pb um $41 \%$ bzw. $30 \%$ signifikant über der Kontrolle. 
Tab.82: Einfluss der $\mathrm{Pb}$-Behandlung (mg/kg Boden) als $\mathrm{Pb}\left(\mathrm{NO}_{3}\right)_{2}$ auf die Konzentration (mg/g TS) und Aufnahme (mg/Topf) von N im Spross von Casuarina equisetifolia

\begin{tabular}{|l|r|r|}
\hline mg Pb/kg Boden & $\begin{array}{l}\text { N-Konzentration } \\
\text { (mg/g TS) }\end{array}$ & \multicolumn{2}{|l|}{$\begin{array}{l}\text { N-Aufnahme } \\
\text { (mg/Topf) }\end{array}$} \\
\hline 0,0 & 8,2 & 57,8 \\
2,5 & 7,8 & 57,7 \\
5 & 9,9 & 81,7 \\
10 & 8,1 & 75,2 \\
20 & 7,9 & 61,5 \\
40 & 7,7 & 59,4 \\
\hline GD 5 \% & 0,8 & 10,0 \\
\hline
\end{tabular}

$\mathrm{P}$-Konzentration und -Aufnahme

Die P-Konzentration im Spross der Casuarina-Pflanzen nahm mit zunehmender $\mathrm{Pb}$ Konzentration im Boden leicht zu (Tab.83). Die signifikant höchste P-Konzentration wurde, anders als beim STG, bei der Pb-Stufe $20 \mathrm{mg} / \mathrm{kg}$ Boden ermittelt. Die P-Aufnahme verlief im wesentlichen entsprechend dem Wachstum. Die höchste P-Aufnahme korrelierte mit dem höchsten STG bei Zugabe von 10 ppm Pb. Sie lag um 50 \% signifikant höher als in der Kontrolle. Die P-Aufnahme bei der höchsten Pb-Stufe 40 mg/kg stieg um $33 \%$ im Vergleich zur Kontrolle ebenfalls signifikant an. 
Tab.83: Einfluss der $\mathrm{Pb}$-Behandlung (mg/kg Boden) als $\mathrm{Pb}\left(\mathrm{NO}_{3}\right)_{2}$ auf die Konzentration (mg/g TS) und Aufnahme (mg/Topf) von P, K, Ca und $\mathrm{Mg}$ im Spross von Casuarina equisetifolia

\begin{tabular}{|c|c|c|c|c|c|c|c|c|}
\hline \multirow[t]{2}{*}{ mg Pb/kg Boden } & \multicolumn{4}{|c|}{ Konzentration (mg/g TS) } & \multicolumn{4}{|c|}{ Aufnahme (mg/Topf) } \\
\hline & $\mathrm{P}$ & $\mathrm{K}$ & $\mathrm{Ca}$ & $\mathrm{Mg}$ & $\mathrm{P}$ & $\mathrm{K}$ & $\mathrm{Ca}$ & $\mathrm{Mg}$ \\
\hline 0,0 & 1,5 & 8,7 & 2,0 & 1,3 & 10,8 & 61,5 & 14,3 & 9,5 \\
\hline 2,5 & 1,7 & 7,2 & 2,3 & 1,2 & 12,4 & 52,8 & 17,2 & 8,7 \\
\hline 5 & 1,8 & 7,5 & 2,2 & 1,4 & 14,8 & 62,1 & 18,0 & 11,6 \\
\hline 10 & 1,7 & 7,2 & 2,7 & 1,3 & 16,3 & 66,8 & 25,1 & 11,9 \\
\hline 20 & 2,0 & 6,3 & 2,2 & 2,5 & 15,0 & 48,5 & 16,5 & 18,3 \\
\hline 40 & 1,9 & 7,5 & 2,4 & 2,2 & 14,4 & 57,9 & 18,1 & 16,5 \\
\hline GD $5 \%$ & 0,4 & 0,9 & ns & 1,0 & 3,4 & 7,8 & 6,6 & 7,0 \\
\hline
\end{tabular}

K-Konzentration und -Aufnahme

Die K-Konzentration sank mit steigender Pb-Konzentration im Boden signifikant zur Kontrolle ab (Tab.83). Die K-Aufnahmen bei den Pb-Stufen 2,5 und $20 \mathrm{mg} / \mathrm{kg}$ Boden lagen signifikant niedriger als bei der Kontrolle. Bei den anderen Pb-Stufen waren die Unterschiede der K-Aufnahme im Vergleich zur Kontrolle nicht signifikant.

Ca-Konzentration und -Aufnahme

Die Pb-Zugaben hatten eine schwachen Einfluss auf die Ca-Konzentrationen im Spross von C. equisetifolia und somit entsprach die Ca-Aufnahme weitgehend dem Wachstum (Tab.83).

Mg-Konzentration und -Aufnahme

Die Mg-Konzentration im Spross nahm bei beiden höchsten Pb-Stufen 20 und 40 ppm zu (Tab. 83). Die signifikant höchste Mg-Konzentration wurde bei der Pb-Stufe $20 \mathrm{mg} / \mathrm{kg}$ Boden festgestellt. Die Mg-Aufnahme entsprach im wesentlichen dem Wachstum. Die höchsten Werte wurden bei den zwei höchsten Pb-Stufen 20 und $40 \mathrm{mg} / \mathrm{kg}$ Boden ermittelt und lagen um $93 \%$ und $74 \%$ signifikant über der Kontrolle.

$\mathrm{Pb}$-Konzentration und -Aufnahme

Wie zu erwarten und wie bei den Acacia-Pflanzen, wurde die Pb-Konzentration im Spross der Casuarina-Pflanzen bei allen $\mathrm{Pb}$-Stufen im Vergleich zur Kontrolle deutlich erhöht (Tab.84). Die höchste Pb-Zugabe $40 \mathrm{mg} / \mathrm{kg}$ Boden erhöhte die Pb-Konzentration auf das 
9fache verglichen mit der Kontrolle. Wegen der erhöhten Pb-Konzentration und des verbesserten Wachstums wiesen alle $\mathrm{Pb}$-Stufen höhere $\mathrm{Pb}$-Aufnahmen als die Kontrolle auf. Die höchste $\mathrm{Pb}$-Zugabe $40 \mathrm{mg} / \mathrm{kg}$ Boden führte zu einer Steigerung der $\mathrm{Pb}$-Aufnahme auf das 11 fache der Kontrolle.

Tab.84: Einfluss der Pb-Behandlung (mg/kg Boden) als $\mathrm{Pb}\left(\mathrm{NO}_{3}\right)_{2}$ auf die Konzentration (mg/g TS) und Aufnahme (mg/Topf) von Pb im Spross von Casuarina equisetifolia

\begin{tabular}{|l|r|r|}
\hline mg Pb/kg Boden & $\begin{array}{c}\text { Pb-Konzentration } \\
\text { (mg/g TS })\end{array}$ & $\begin{array}{c}\text { Pb-Aufnahme } \\
\text { (mg/Topf })\end{array}$ \\
\hline 0,0 & 0,007 & 0,047 \\
2,5 & 0,013 & 0,097 \\
5 & 0,015 & 0,126 \\
10 & 0,030 & 0,280 \\
20 & 0,063 & 0,479 \\
40 & 0,066 & 0,502 \\
\hline GD 5\% & 0,015 & 0,083 \\
\hline
\end{tabular}

\subsubsection{Pb-Konzentration und -Aufnahme in der Wurzel}

Die Wurzeln zeigten im wesentlichen das gleiche Bild wie die Sprosse, jedoch waren alle Werte der Pb-Konzentration und $\mathrm{Pb}$-Aufnahme viel höher (Tab.85). Bei der höchsten $\mathrm{Pb}$ Stufe 40 ppm lag die Pb-Konzentration 110fach höher als in der Kontrolle. In dieser $\mathrm{Pb}$ Behandlung wurde die Pb-Aufnahme in die Wurzeln auf das 164fache gegenüber der Kontrolle gesteigert. 
Tab.85: Einfluss der $\mathrm{Pb}-$ Behandlung (mg/kg Boden) als $\mathrm{Pb}\left(\mathrm{NO}_{3}\right)_{2}$ auf die Konzentration (mg/g TS) und Aufnahme (mg/Topf) von $\mathrm{Pb}$ in der Wurzel von Casuarina equisetifolia

\begin{tabular}{|l|r|r|}
\hline mg Pb/kg Boden & $\begin{array}{l}\text { Pb-Konzentration } \\
\text { (mg/g TS) }\end{array}$ & $\begin{array}{l}\text { Pb-Aufnahme } \\
\text { (mg/Topf) }\end{array}$ \\
\hline 0,0 & 0,005 & 0,008 \\
2,5 & 0,048 & 0,121 \\
5 & 0,115 & 0,313 \\
10 & 0,182 & 0,479 \\
20 & 0,381 & 0,955 \\
40 & 0,548 & 1,315 \\
\hline GD 5\% & 0,107 & 0,278 \\
\hline
\end{tabular}

\subsubsection{Der pH-Wert des Bodens}

Der pH-Wert im Boden zeigte bei C. equisetifolia in den $\mathrm{Pb}$-Behandlungen eine sehr ähnliche Tendenz wie im ersten Versuch, alle $\mathrm{pH}$-Werte lagen hier jedoch etwas niedriger als im 1. Versuch (Tab.86). Zwischen den Pb-Stufen und der Kontrolle ergaben sich keine signifikanten Unterschiede. Der Ausgang-pH-Wert sank am Ende des Versuchs von 7 bis auf 4,9 ab.

Tab.86: Einfluss der $\mathrm{Pb}$-Behandlung (mg/kg Boden) als $\mathrm{Pb}\left(\mathrm{NO}_{3}\right)_{2}$ auf den pH-Wert im Boden von Casuarina equisetifolia am Ende des Versuches

\begin{tabular}{|l|l|l|l|l|l|l|l|}
\hline $\mathrm{mg} \mathrm{Pb} / \mathrm{kg}$ Boden & \multicolumn{1}{|c|}{0,0} & 2,5 & 5 & 10 & 20 & 40 & GD 5\% \\
\hline $\mathrm{pH}$ & 5,0 & 5,1 & 5,0 & 4,9 & 5,0 & 5,0 & $\mathrm{~ns}$ \\
\hline
\end{tabular}




\subsubsection{Cupressus sempervirens}

\subsubsection{Sprosstrockengewicht (STG)}

Die steigende $\mathrm{Pb}$-Zugaben hatten einen deutlich positiven Einfluss auf das STG (Tab.87). Zwischen der Kontrolle und den Pb-Stufen 5, 10 und 20 ppm waren signifikante Steigerungen festzustellen. Das höchste STG wurde bei den Pb-Behandlungen 10 und 20 ppm ermittelt, es lag um 68 \% über der Kontrolle. Bei der höchsten Pb-Stufe 40 ppm war das STG zwar um 36 \% höher, jedoch nicht mehr signifikant verschieden von der Kontrolle.

Tab.87: Einfluss der Pb-Behandlung (mg/kg Boden) als $\mathrm{Pb}\left(\mathrm{NO}_{3}\right)_{2}$ auf das Sprosstrockengewicht (g/Topf) von Cupressus sempervirens

\begin{tabular}{|l|l|l|l|l|l|l|l|}
\hline $\mathrm{mg} \mathrm{Pb} / \mathrm{kg}$ Boden & 0,0 & 2,5 & 5 & 10 & 20 & 40 & GD 5\% \\
\hline STG g/Topf & 2,8 & 3,4 & 4,2 & 4,7 & 4,7 & 3,8 & 1,1 \\
\hline
\end{tabular}

\subsubsection{Wurzeltrockengewicht (WTG)}

Das WTG zeigte einen ähnlichen Verlauf wie das STG (Tab. 88). Der höchste positive Effekt der Pb-Behandlung wurde bei der Pb-Stufe $20 \mathrm{mg} / \mathrm{kg}$ Boden festgestellt. Hier lag das WTG fast 3fach höher als in der Kontrolle. Für das WTG ergaben sich bei den PbStufen 5, 10, 20 und $40 \mathrm{mg} / \mathrm{kg}$ Boden signifikante Steigerungen gegenüber der Kontrolle. Die höchste Pb-Zugabe $40 \mathrm{mg} / \mathrm{kg}$ Boden förderte das WTG auf das Doppelte im Vergleich zur Kontrolle.

Tab.88: Einfluss der $\mathrm{Pb}$-Behandlung (mg/kg Boden) als $\mathrm{Pb}\left(\mathrm{NO}_{3}\right)_{2}$ auf das Wurzeltrockengewicht (g/Topf) von Cupressus sempervirens

\begin{tabular}{|l|l|l|l|l|l|l|l|}
\hline mg Pb/kg Boden & 0,0 & 2,5 & 5 & 10 & 20 & 40 & GD 5 \% \\
\hline WTG g/Topf & 0,5 & 0,6 & 0,9 & 1,0 & 1,3 & 1,0 & 0,3 \\
\hline
\end{tabular}

5.2.3.3 Konzentration und Aufnahme von $\mathrm{N}, \mathrm{P}, \mathrm{K}, \mathrm{Ca}, \mathrm{Mg}$ und $\mathrm{Pb}$ im Spross

$\mathrm{N}$-Konzentration und -Aufnahme

Die Zunahme der Pb-Konzentration im Boden führte $\mathrm{zu}$ einer Verringerung der $\mathrm{N}$ Akkumulation im Spross von C. sempervirens (Tab.89). Zwischen den PbBehandlungen 2,5; 5; 20 und 40 ppm und der Kontrolle ergaben sich keine signifikanten 
Verringerungen der $\mathrm{N}$-Konzentration. Lediglich die $\mathrm{Pb}$-Stufe $10 \mathrm{mg} / \mathrm{kg}$ Boden rief eine signifikante Abnahme der N-Konzentration im Vergleich zur Kontrolle hervor. Die NAufnahme entsprach weitgehend dem Wachstum. Sie lag bei allen $\mathrm{Pb}$-Stufen zwar höher als die Kontrolle, jedoch nur bei der Pb-Stufe $5 \mathrm{mg} / \mathrm{kg}$ Boden war die Steigerung der $\mathrm{N}$ Aufnahme signifikant um etwa $45 \%$ höher als in der Kontrolle.

Tab.89: Einfluss der $\mathrm{Pb}$-Behandlung (mg/kg Boden) als $\mathrm{Pb}\left(\mathrm{NO}_{3}\right)_{2}$ auf die Konzentration (mg/g TS) und Aufnahme (mg/Topf) von N im Spross von Cupressus sempervirens

\begin{tabular}{|l|r|r|}
\hline mg Pb/kg Boden & $\begin{array}{c}\text { N-Konzentration } \\
\text { (mg/g TS })\end{array}$ & $\begin{array}{l}\text { N-Aufnahme } \\
\text { (mg/Topf })\end{array}$ \\
\hline 0,0 & 13,5 & 37,9 \\
2,5 & 12,1 & 40,6 \\
5 & 13,2 & 55,0 \\
10 & 11,2 & 53,0 \\
20 & 11,9 & 54,6 \\
40 & 13,0 & 50,0 \\
\hline GD $5 \%$ & 2,1 & 16,9 \\
\hline
\end{tabular}

P-Konzentration und -Aufnahme

Die P-Konzentration im Spross zeigte keine bedeutenden Unterschiede zwischen der Kontrolle und allen $\mathrm{Pb}$-Stufen (Tab.90). Sie stieg mit zunehmender Pb-Gabe zum Boden bis zu $5 \mathrm{mg} / \mathrm{kg}$ Boden nur leicht an; danach nahm sie ab. Steigende $\mathrm{Pb}$-Zugaben führten aufgrund des höheren TG zu höheren P-Aufnahmen. Zwischen den $\mathrm{Pb}$-Stufen und der Kontrolle waren die Unterschiede jedoch nicht signifikant. 
Tab. 90: Einfluss der $\mathrm{Pb}$-Behandlung (mg/kg Boden) als $\mathrm{Pb}\left(\mathrm{NO}_{3}\right)_{2}$ auf die Konzentration (mg/g TS) und Aufnahme (mg/Topf) von P, K, Ca und $\mathrm{Mg}$ im Spross von Cupressus sempervirens

\begin{tabular}{|l|llll|lllll|}
\hline \multirow{2}{*}{$\mathrm{mg} \mathrm{Pb} / \mathrm{kg}$ Boden } & \multicolumn{6}{|l|}{ Konzentration $(\mathrm{mg} / \mathrm{g}$ TS) } & \multicolumn{4}{|c|}{ Aufnahme (mg/Topf) } \\
\cline { 2 - 10 } & $\mathrm{P}$ & $\mathrm{K}$ & $\mathrm{Ca}$ & $\mathrm{Mg}$ & $\mathrm{P}$ & $\mathrm{K}$ & $\mathrm{Ca}$ & $\mathrm{Mg}$ \\
\hline 0,0 & 1,2 & 12,1 & 6,5 & 1,5 & 3,4 & 33,9 & 18,3 & 4,1 \\
2,5 & 1,4 & 10,7 & 5,3 & 1,1 & 4,8 & 36,9 & 18,2 & 3,7 \\
5 & 1,4 & 10,9 & 4,6 & 1,1 & 5,7 & 45,3 & 18,8 & 4,4 \\
10 & 1,2 & 9,1 & 3,8 & 1,3 & 5,7 & 42,9 & 18,1 & 6,0 \\
20 & 1,1 & 11,2 & 3,3 & 1,3 & 5,1 & 51,9 & 14,9 & 6,0 \\
40 & 1,0 & 10,4 & 4,8 & 1,4 & 3,6 & 40,5 & 17,8 & 5,3 \\
\hline \multirow{2}{*}{ GD 5\% } & $\mathrm{ns}$ & 2,6 & 1,3 & 0,2 & $\mathrm{~ns}$ & 16,9 & $\mathrm{~ns}$ & 1,7 \\
\hline
\end{tabular}

K-Konzentration und -Aufnahme

Insgesamt nahm die $\mathrm{K}$-Konzentration durch die steigende $\mathrm{Pb}$-Konzentration im Boden bis $10 \mathrm{mg} / \mathrm{kg}$ Boden ab und danach ab der Pb-Stufe $20 \mathrm{mg} / \mathrm{kg}$ Boden wieder zu (Tab.90). Alle Werte lagen jedoch niedriger als bei der Kontrolle. Die Pb-Zugabe von $10 \mathrm{ppm}$ führte zu einer signifikanten Verringerung der K-Konzentration um ca. $25 \%$ gegenüber der Kontrolle. Bei der höchsten Pb-Stufe 40 ppm lag die K-Konzentration um etwa $14 \%$ niedriger als in der Kontrolle. Die K-Aufnahme entsprach im wesentlichen dem Wachstum. Sie stieg gegenüber der Kontrolle bei allen $\mathrm{Pb}$-Stufen an. Nur bei der Pb-Stufe $20 \mathrm{mg} / \mathrm{kg}$ Boden lag die K-Aufnahme um $53 \%$ signifikant über der Kontrolle.

Ca-Konzentration und -Aufnahme

Die Ca-Konzentration nahm im Spross mit steigender Pb-Zugabe zum Boden verglichen mit der Kontrolle ab (Tab. 90). Die niedrigste Ca-Akkumulation wurde bei der Pb-Stufe $20 \mathrm{mg} / \mathrm{kg}$ Boden mit $49 \%$ signifikant unter der Kontrolle ermittelt. Weitere signifikante Verringerungen der Ca-Konzentrationen der Pflanzen wurden bei 5, 10 und $40 \mathrm{mg} \mathrm{Pb} / \mathrm{kg}$ Boden gegenüber der Kontrolle festgestellt. Die Ca-Aufnahme war in allen Behandlungen trotz dem verbesserten Wachstum der Pflanzen nach Pb-Zugabe nicht signifikant verschieden. 


\section{Mg-Konzentration und -Aufnahme}

Die Mg-Konzentrationen aller Pb-Stufen lagen niedriger als die der Kontrolle (Tab.90). Signifikante Unterschiede wurden nur bei den $\mathrm{Pb}$-Stufen 2,5 und $5 \mathrm{mg} / \mathrm{kg}$ Boden festgestellt. Die Mg-Aufnahme im Spross entsprach im wesentlichen dem STG. Nur bei Zugabe von 10 bzw. 20 ppm war die Mg-Aufnahme signifikant höher als die Kontrolle.

$\mathrm{Pb}$-Konzentration und -Aufnahme

Wie die Acacia- und Casuarina-Pflanzen akkumulierten auch die Cupressus-Pflanzen in ihrem Spross viel höhere $\mathrm{Pb}$-Mengen bei allen $\mathrm{Pb}$-Behandlungen als in der Kontrolle (Tab.91). Bei der höchsten Pb-Gabe $40 \mathrm{mg} / \mathrm{kg}$ Boden lag die Pb-Konzentration 13-mal so hoch wie in der Kontrolle. Aufgrund des relativ hohen TG und der gesteigerten $\mathrm{Pb}$ Konzentration lag die $\mathrm{Pb}$-Aufnahme im Spross bei den $\mathrm{Pb}$-Behandlungen wesentlich höher als in der Kontrolle. Die Zufuhr von 40 ppm erhöhte die Pb-Aufnahme auf das 18fache gegenüber der Kontrolle.

Tab.91: Einfluss der $\mathrm{Pb}$-Behandlung (mg/kg Boden) als $\mathrm{Pb}\left(\mathrm{NO}_{3}\right)_{2}$ auf die Konzentration (mg/g TS) und Aufnahme (mg/Topf) von $\mathrm{Pb}$ im Spross von Cupressus sempervirens

\begin{tabular}{|l|r|r|}
\hline mg Pb/kg Boden & $\begin{array}{l}\text { Pb-Konzentration } \\
\text { (mg/g TS) }\end{array}$ & $\begin{array}{l}\text { Pb-Aufnahme } \\
\text { (mg/Topf) }\end{array}$ \\
\hline 0,0 & 0,006 & 0,016 \\
2,5 & 0,012 & 0,041 \\
5 & 0,017 & 0,072 \\
10 & 0,021 & 0,097 \\
20 & 0,030 & 0,138 \\
40 & 0,078 & 0,287 \\
\hline GD 5\% & 0,007 & 0,067 \\
\hline
\end{tabular}

\subsubsection{Pb-Konzentration und -Aufnahme in der Wurzel}

Die Pb-Akkumulation in den Wurzeln die Cupressus-Pflanzen wies im allgemeinen eine ähnliche Tendenz auf wie in den Sprossen (Tab.92). Die Werte lagen jedoch viel höher als in den Sprossen. Die höchste Pb-Konzentration wurde bei der höchsten Pb-Stufe $40 \mathrm{mg} / \mathrm{kg}$ Boden ermittelt. Sie war 213fach höher als in der Kontrolle. Die Pb-Aufnahme lag bei allen 
$\mathrm{Pb}$-Stufen wegen des verbesserten Wachstums und der hohen $\mathrm{Pb}$-Konzentration höher als in der Kontrolle. Bei der höchsten Pb-Behandlung $40 \mathrm{ppm}$ wurde sie auf das 450fache gegenüber der Kontrolle erhöht.

Tab.92: Einfluss der $\mathrm{Pb}$-Behandlung (mg/kg Boden) als $\mathrm{Pb}\left(\mathrm{NO}_{3}\right)_{2}$ auf die Konzentration (mg/g TS) und Aufnahme (mg/Topf) von $\mathrm{Pb}$ in der Wurzel von Cupressus sempervirens

\begin{tabular}{|l|r|r|}
\hline mg Pb/kg Boden & $\begin{array}{l}\text { Pb-Konzentration } \\
(\mathrm{mg} / \mathrm{g} \text { TS })\end{array}$ & $\begin{array}{l}\text { Pb-Aufnahme } \\
\text { (mg/Topf })\end{array}$ \\
\hline 0,0 & 0,006 & 0,003 \\
2,5 & 0,066 & 0,041 \\
5 & 0,168 & 0,155 \\
10 & 0,346 & 0,335 \\
20 & 0,620 & 0,792 \\
40 & 1,280 & 1,351 \\
\hline GD $5 \%$ & 0,225 & 0,450 \\
\hline
\end{tabular}

\subsubsection{Der $\mathrm{pH}-$ Wert des Bodens}

Wie bei den anderen zwei Baumarten zeigte sich bei den Cupressus-Pflanzen, dass die pHWerte im Boden nach Behandlung mit $\mathrm{Pb}$ am Ende des Versuches von 7 bis auf 4,7 abnahmen (Tab.93). Die pH-Werte wiesen ein ähnliches Bild wie im ersten Versuch auf, waren jedoch etwas höher und unterschieden sich nicht signifikant von der Kontrolle.

Tab. 93: Einfluss der Pb-Behandlung (mg/kg Boden) als $\mathrm{Pb}\left(\mathrm{NO}_{3}\right)_{2}$ auf den pH-Wert im Boden von Cupressus sempervirens am Ende des Versuches

\begin{tabular}{|l|r|r|r|r|r|r|l|}
\hline $\mathrm{mg} \mathrm{Pb} / \mathrm{kg}$ Boden & 0,0 & 2,5 & 5 & 10 & 20 & 40 & GD 5\% \\
\hline $\mathrm{pH}$ & 4,9 & 5,0 & 4,7 & 4,9 & 4,8 & 4,9 & $\mathrm{~ns}$ \\
\hline
\end{tabular}




\section{Zusammenfassender Überblick über die Ergebnisse von Versuch 2}

Das Ziel dieses Versuches war, die Wirkung der Pb-Zugabe zum Boden in Form von $\mathrm{Pb}\left(\mathrm{NO}_{3}\right)_{2}$, jedoch anders als im 1. Versuch, unter Berücksichtigung des in diesem Salz enthaltenen Stickstoffs bei den drei Baumarten des 1. Versuches, zu prüfen, um eine Nebenwirkung dieses begleitenden $\mathrm{N}$ auszuschließen. Hierfür wurde bei jeder $\mathrm{Pb}$ Behandlung die N-Menge des Bleinitrats von der vorgesehenen N-Düngungsmenge abgezogen.

Im allgemeinen zeigten die Pflanzen der drei Baumarten bei der Anwendung des $\mathrm{Pb}\left(\mathrm{NO}_{3}\right)_{2}$ eine ähnliche Tendenz des Wachstums wie im 1.Versuch. Lediglich lagen die STG bzw. WTG sowie die Konzentrationen bzw. Aufnahmen der Nährstoffe in den Sprossen etwas höher als bei den entsprechenden $\mathrm{Pb}$-Stufen des 1.Versuch. Dies kann darauf zurückgeführt werden, dass beide Versuche zu verschiedenen Jahreszeiten bzw. unter verschiedenen Temperatur- und Lichtverhältnissen durchgeführt wurden.

Aus Tab.94 geht hervor, dass die Pflanzen der drei Baumarten eine Pb-Zugabe von $10 \mathrm{mg} / \mathrm{kg}$ Boden tolerierten und höchste Sprosstrockenmaße produzierten; die CupressusPflanzen erreichten sogar das höchste STG bei Zufuhr von 20 ppm Pb. Bei der TS-Bildung in den Wurzeln wiesen die Acacia-Pflanzen, anders als beim Spross, das höchste WTG bei der niedrigeren $\mathrm{Pb}$-Stufe von $5 \mathrm{mg} / \mathrm{kg}$ Boden auf. Die Casuarina-Pflanzen zeigten ein breites Spektrum der Pb-Behandlung von 2,5 bis $20 \mathrm{mg} / \mathrm{kg}$ Boden zur Bildung höherer WTG. Das höchste WTG der Cupressus-Pflanzen wurde bei der hohen Pb-Zugabe von 20 $\mathrm{mg} / \mathrm{kg}$ Boden ermittelt.

Die höchsten Aufnahmen der Acacia-Pflanzen an N, P, K und Mg im Spross korrelierten mehr oder weniger mit den $\mathrm{Pb}$-Behandlungen, in denen die höchsten Trockenmassen im Spross gebildet wurden. Ähnlich verhielt sich die Nährstoffaufnahme im Spross der Casuarina-Pflanzen, mit Ausnahme der bei einer (im Vergleich zum Spross) niedrigen $\mathrm{Pb}$ Behandlung von $5 \mathrm{mg} / \mathrm{kg}$ Boden höchsten $\mathrm{N}$-Aufnahme, und bei einer höchsten $\mathrm{Pb}$ Behandlung von $20-40 \mathrm{mg} / \mathrm{kg}$ Boden ermittelten höchsten $\mathrm{Mg}$-Aufnahme. Die höchsten Nährstoffaufnahmen im Spross der Cupressus-Pflanzen korrelierten mehr oder weniger mit dem Wachstum. 
Tab.94: Pb-Applikationen die zu den höchsten Spross-, Wurzel-Massen und Nährstoffaufnahmen im Spross führten (Optimale Wachstumbedingungen) in diesem Versuch

\begin{tabular}{|c|c|c|c|c|c|c|c|}
\hline \multirow[t]{3}{*}{ Baum } & \multicolumn{7}{|l|}{ Höchste } \\
\hline & $\begin{array}{l}\text { Spoßmasse } \\
\text { (g/Topf) }\end{array}$ & $\begin{array}{l}\text { Wurzelmasse } \\
\text { (g/Topf) }\end{array}$ & $\begin{array}{l}\text { N-Aufnahme } \\
\text { (mg/Topf) }\end{array}$ & $\begin{array}{l}\text { P-Aufnahme } \\
\text { (mg/Topf) }\end{array}$ & $\begin{array}{l}\text { K-Aufnahme } \\
\text { (mg/Topf) }\end{array}$ & $\begin{array}{l}\text { Ca-Aufnahme } \\
\text { (mg/Topf) }\end{array}$ & $\begin{array}{l}\text { Mg-Aufnahme } \\
\text { (mg/Topf) }\end{array}$ \\
\hline & \multicolumn{7}{|c|}{$\mathrm{Pb}$-Zugabe (mg/kg Boden) } \\
\hline Acacia & 10 & 5 & $2,5-10$ & $5-20$ & $2,5-20 / 40$ & $5-20$ & 5- 10 \\
\hline Casuarina & 10 & $2,5-20$ & 5 & $10-20$ & 10 & 10 & $20-40$ \\
\hline Cupressus & $10-20$ & 20 & $5-20$ & $5-10$ & 20 & $0-40$ & $10-20$ \\
\hline
\end{tabular}

Tabelle 95 zeigt, dass sich sogar die höchste Pb-Behandlung 40 ppm fördernd auf das STG und WTG der drei Baumarten im Vergleich zur jeweiligen Kontrolle auswirkte. Obwohl die Pflanzen im allgemeinen sehr hohe Mengen an $\mathrm{Pb}$ bei dieser $\mathrm{Pb}$-Stufe akkumulierten (mg/g TS) bzw. aufnahmen (mg/Topf) (Tab.77-78; 84-85 und 91-92), wurden die Nährstoffaufnahmen im Spross der drei Baumarten, mit Ausnahme der schwachen Abnahme der NAufnahme der Acacia-Pflanzen, entweder nicht bedeutend beeinflusst (K bei C. equisetifolia und $\mathrm{Ca}$ bei $C$. sempervirens) oder deutlich gefördert gegenüber den Kontrollpflanzen.

In anderen Worten, die Pflanzen haben nicht nur dem Boden hohe Mengen des Schwermetalls $\mathrm{Pb}$ entnommen (de facto bei allen Pb-Zugaben zum Boden), sondern wiesen ebenfalls ein besseres Wachstum begleitet mit meist erhöhter Aufnahme der Makronährstoffen auf. 
Tab. 95: Die Zunahmen und Abnahmen in \% des STG und WTG sowie der Aufnahme von N, P, K, Ca, und Mg im Spross der höchsten PbZugabe $40 \mathrm{mg} / \mathrm{kg}$ Boden gegenüber der jeweiligen Kontrolle

\begin{tabular}{|c|c|c|c|c|c|c|c|}
\hline \multirow[t]{2}{*}{ Baum } & \multirow[b]{2}{*}{ STG } & \multirow[b]{2}{*}{ WTG } & \multicolumn{5}{|c|}{ Aufnahme (mg/Topf) von } \\
\hline & & & $\mathrm{N}$ & $P$ & $\mathrm{~K}$ & $\mathrm{Ca}$ & $\mathrm{Mg}$ \\
\hline Acacia & $\begin{array}{l}\uparrow+ \\
67,2\end{array}$ & $\begin{array}{l}\uparrow \\
9,0\end{array}$ & $\begin{array}{l}\downarrow \\
17,6\end{array}$ & \begin{tabular}{|l}
$\uparrow$ \\
43,0
\end{tabular} & $\begin{array}{l}\uparrow+ \\
39,4\end{array}$ & $\begin{array}{l}\uparrow \\
66,6\end{array}$ & $\begin{array}{l}\uparrow \\
48,0\end{array}$ \\
\hline Casuarina & $\begin{array}{l}\uparrow \\
9,1\end{array}$ & $\begin{array}{l}\uparrow \\
41,0\end{array}$ & $\begin{array}{l}\uparrow \sim ~ \\
2,6\end{array}$ & $\begin{array}{l}\uparrow+ \\
33,6\end{array}$ & $\begin{array}{l}\downarrow \sim \\
5,8\end{array}$ & $\begin{array}{l}\uparrow \\
26,7\end{array}$ & $\begin{array}{l}\uparrow+ \\
74,7\end{array}$ \\
\hline Cupressus & $\begin{array}{l}\uparrow \\
34,4\end{array}$ & $\begin{array}{l}\uparrow+ \\
124,4\end{array}$ & $\begin{array}{l}\uparrow \\
32,1\end{array}$ & $\begin{array}{l}\uparrow \sim ~ \\
4,7\end{array}$ & $\begin{array}{l}\uparrow \\
19,5\end{array}$ & $\begin{array}{l}\downarrow \sim \\
3,2\end{array}$ & $\begin{array}{l}\uparrow \\
27,4\end{array}$ \\
\hline
\end{tabular}

\footnotetext{
$\uparrow:$ Höher $\downarrow$ : ni edri ger

$\sim$ : Fast gleich geblieben

个+: Si gni fi kant höher
}

$\downarrow-$ : ni edri ger, aber ni cht si gni fi kant

Tab.96: Das Verhältnis der Werte bei den höchsten bzw. toxischen Pb-Gaben in \% zu den höchsten Werten (optimal =100)

\begin{tabular}{|c|c|c|c|c|c|c|c|}
\hline \multirow[t]{2}{*}{ Baum } & $\begin{array}{l}\text { STG } \\
(\%)\end{array}$ & $\begin{array}{l}\text { WTG } \\
(\%)\end{array}$ & $\begin{array}{l}\mathrm{N}- \\
\text { Aufnahme } \\
\quad(\%)\end{array}$ & $\begin{array}{l}\text { P- } \\
\text { Aufnahme } \\
\quad(\%)\end{array}$ & $\begin{array}{l}\text { K- } \\
\text { Aufnahme } \\
\quad(\%)\end{array}$ & $\begin{array}{l}\text { Ca- } \\
\text { Aufnahme } \\
\quad(\%)\end{array}$ & $\begin{array}{l}\text { Mg- } \\
\text { Aufnahme } \\
\quad(\%)\end{array}$ \\
\hline & \multicolumn{7}{|c|}{ Die Werte bei $\mathrm{HPbG}$ in $\%$ von den Optimalen Werten bei PbG } \\
\hline Acacia & $\begin{array}{l}{ }^{+*} \\
68 \%\end{array}$ & $34 \%$ & $47 \%$ & $61 \%$ & $\begin{array}{l}{ }^{+} * \\
85 \%\end{array}$ & $62 \%$ & $61 \%$ \\
\hline Casuarina & $\underline{83 \%}$ & $\underline{91 \%}$ & $73 \%$ & $\begin{array}{l}{ }^{+} * \\
88 \%\end{array}$ & $87 \%$ & $72 \%$ & $\begin{array}{l}+* \\
90 \%\end{array}$ \\
\hline Cupressus & $79 \%$ & $78 \%^{+*}$ & $91 \%$ & $63 \%$ & $78 \%$ & $95 \%$ & $88 \%$ \\
\hline
\end{tabular}

$\mathrm{PbG}=\mathrm{Pb}$-Gaben $\mathrm{HPbG}=$ Höchste Pb-Gaben

Tabelle 96 stellt das Verhältnis der Werte bei den höchsten bzw. toxischen $\mathrm{Pb}-\mathrm{Gaben}$ in \% zu den höchsten Werten (optimal=100) bei verschiedenen $\mathrm{Pb}-\mathrm{Gaben}$ dar. Die unterstrichenen Werte zeigen die höchste Toleranz der Pflanzen der drei Baumarten in Form der höchsten TSBildung im Spross und in der Wurzel, sowie die Fähigkeit der Pflanzen, Nährstoffe 
aufzunehmen bei den höchsten Schwermetall-Gaben in \% im Vergleich zu den optimalen (höchsten) Werten.

Die pH-Werte im Boden zeigten bei der Pb-Behandlung ein ähnliches Bild wie im ersten Versuch. Die Pb-Behandlung führte zu einer Verringerung des Ausgangs-pH-Wertes zum Ende des Versuches, wobei alle Unterschiede zur Kontrolle nicht signifikant waren (Tab. 79, 86 u.93). 


\subsection{Versuch 3}

Dieser Versuch sollte die Wirkung einer kombinierten Behandlung mit den Schwermetallen $\mathrm{Zn}, \mathrm{Cd}$ oder $\mathrm{Pb}$ und der Inokulation der Pflanzen mit den $\mathrm{N}_{2}$-fixierenden Rhizobien bzw. Actinomyceten (Frankia) auf das Wachstum und die Nährstoffaufnahme von Acacia- (bei Rhizobien) und Casuarina-Pflanzen (bei Frankia) prüfen.

Die Schwermetalle wurden jeweils in vier Stufen dem Boden verabreicht:

Zn 0; 0,5; 10 und $80 \mathrm{mg} / \mathrm{kg}$ Boden

$\mathrm{Cd} 0 ; 1,25 ; 5$ und $20 \mathrm{mg} / \mathrm{kg}$ Boden

$\mathrm{Pb} 0 ; 2,5 ; 10$ und $80 \mathrm{mg} / \mathrm{kg}$ Boden

Da eine Zugabe von 40 ppm an $\mathrm{Zn}$ und /oder $\mathrm{Pb}$ keine deutlich schädigende Wirkung auf das Wachstum im 1. Versuch (beide Schwermetalle) und im 2. Versuch (Pb) gezeigte hatte, wurde diese Stufe jetzt durch die höhere Gabe von $80 \mathrm{mg} / \mathrm{kg}$ Boden ersetzt.

Für die Inokulation der Pflanzen wurde eine Mischung aus zwei Rhizobien-Stämmen (bei A. saligna) bzw. aus zwei Frankia-Stämmen (bei C. equisetifolia) als Inokulum hergestellt und die Pflanzen damit inokuliert.

Die Pflanzen wurden, wie unter 4.4 beschrieben, gedüngt. Weitere Einzelheiten der Versuchsdurchführung sind im Kapitel " Material und Methodik " angegeben.

\subsubsection{Acacia saligna}

\subsubsection{Zn-Behandlung}

\subsection{Sprosstrockengewicht (STG)}

Die Beimpfung förderte das Wachstum von A. saligna bei allen Zn-Stufen hoch signifikant mit Ausnahme der höchsten Zn-Stufe $80 \mathrm{mg} / \mathrm{kg}$ Boden/-R/Pflanzen gegenüber der Kontrolle (Tab.97). Das beste Wachstum der -R/Pflanzen wurde bei der Zn-Stufe $10 \mathrm{mg} / \mathrm{kg}$ Boden ermittelt, dieser Wert unterschied sich hoch signifikant von der Kontrolle und der höchsten Zn-Stufe $80 \mathrm{mg} / \mathrm{kg}$ Boden. Das höchste STG der +R/Pflanzen wurde ebenfalls bei der ZnStufe $10 \mathrm{mg} / \mathrm{kg}$ Boden festgestellt und lag fast 7-mal so hoch wie in der Kontrolle und fast 3mal so hoch wie bei den -R/Pflanzen. Die höchste Zn-Stufe $80 \mathrm{mg} / \mathrm{kg}$ Boden führte zu einer Steigerung des STG der +R/Pflanzen auf das 3,3fache gegenüber der Kontrolle und auf das 7 fache gegenüber den -R/Pflanzen. Mit steigender Zn-Stufe bis zu $80 \mathrm{mg} / \mathrm{kg}$ Boden nahm das Wachstum der $-\mathrm{R} /$ und $+\mathrm{R} /$ Pflanzen im Vergleich zu den Zn-Stufen 0,5 und $10 \mathrm{mg} / \mathrm{kg}$ Boden deutlich ab. In anderen Worten, die höchste Zn-Zugabe $80 \mathrm{mg} / \mathrm{kg}$ Boden wirkte fördernd auf das Wachstum der Acacia-Pflanzen, jedoch nur wenn die Pflanzen mit Rhizobien inokuliert waren. 
Tab.97: Einfluss der Zn-Behandlung (mg/kg Boden) ohne (-R) oder mit Rhizobien (+R) auf das Sprosstrockengewicht (g/Topf) von Acacia saligna

\begin{tabular}{|l|l|l|l|l|l|}
\hline mg Zn/kg Boden & 0,0 & 0,5 & 10 & 80 & GD 5\% \\
\hline $\begin{array}{l}\text { STG g/Topf } \\
-\mathrm{R}\end{array}$ & 0,5 & 2,1 & 3,5 & 0,7 & \\
$+\mathrm{R}$ & 1,5 & 7,5 & 9,9 & 3,0 & 1,5 \\
\hline
\end{tabular}

\subsection{Wurzeltrockengewicht (WTG)}

Das WTG aller Varianten zeigte einen fast identischen Verlauf wie das STG, nur lagen die Werte deutlich niedriger (Tab.98). Ebenfalls anders als beim Spross war das WTG der +R/Pflanzen bei der höchsten Zn-Stufe $80 \mathrm{mg} / \mathrm{kg}$ Boden geringer als in der Kontrolle.

Tab.98: Einfluss der Zn-Behandlung (mg/kg Boden) ohne (-R) oder mit Rhizobien (+R) auf das Wurzeltrockengewicht (g/Topf) von Acacia saligna

\begin{tabular}{|l|l|l|l|l|l|}
\hline mg Zn/kg Boden & 0,0 & 0,5 & 10 & 80 & GD 5\% \\
\hline $\begin{array}{l}\text { WTG g/Topf } \\
-\mathrm{R}\end{array}$ & 0,2 & 0,9 & 1,5 & 0,3 & \\
$+\mathrm{R}$ & 0,6 & 3,0 & 3,9 & 0,4 & 0,4 \\
\hline
\end{tabular}

\subsection{Konzentration und Aufnahme von N, P, K, Ca, Mg und Zn im Spross}

\section{$\mathrm{N}$-Konzentration und -Aufnahme}

Die N-Konzentrationen ließen keine bedeutenden Unterschiede zwischen inokulierten und nicht inokulierten Pflanzen erkennen (Tab.99). Jedoch lag die N-Konzentration der inokulierten Kontrolle höher als die der -R/Pflanzen. Bei der höchsten Zn-Stufe 80 ppm nahm die N-Konzentration im Spross der $-\mathrm{R} /$ und besonders $+\mathrm{R} /$ Pflanzen im Vergleich zur Kontrolle ab. Die N-Aufnahme der $+\mathrm{R} /$ und $-\mathrm{R} / \mathrm{Pflanzen}$ entsprach im wesentlichen dem Wachstum. Insgesamt nahmen die N-Aufnahmen der $+\mathrm{R} /$ und -R/Pflanzen mit steigenden $\mathrm{Zn}$ Gaben zunächst zu und später bei der höchsten Zn-Stufe $80 \mathrm{mg} / \mathrm{kg}$ Boden wieder ab. Die NAufnahme wurde durch die Beimpfung der Acacia-Pflanzen auf das 3fache (bei 0,5 und 10 $\mathrm{mg} / \mathrm{kg}$ Boden) bis auf das 5fache (bei der Kontrolle) gegenüber den unbeimpften Pflanzen gesteigert. 
Tab.99: Einfluss der Zn-Behandlung (mg/kg Boden) ohne (-R) oder mit Rhizobien (+R) auf die Konzentration (mg/g TS) und Aufnahme (mg/Topf) von $\mathrm{N}$ im Spross von Acacia saligna

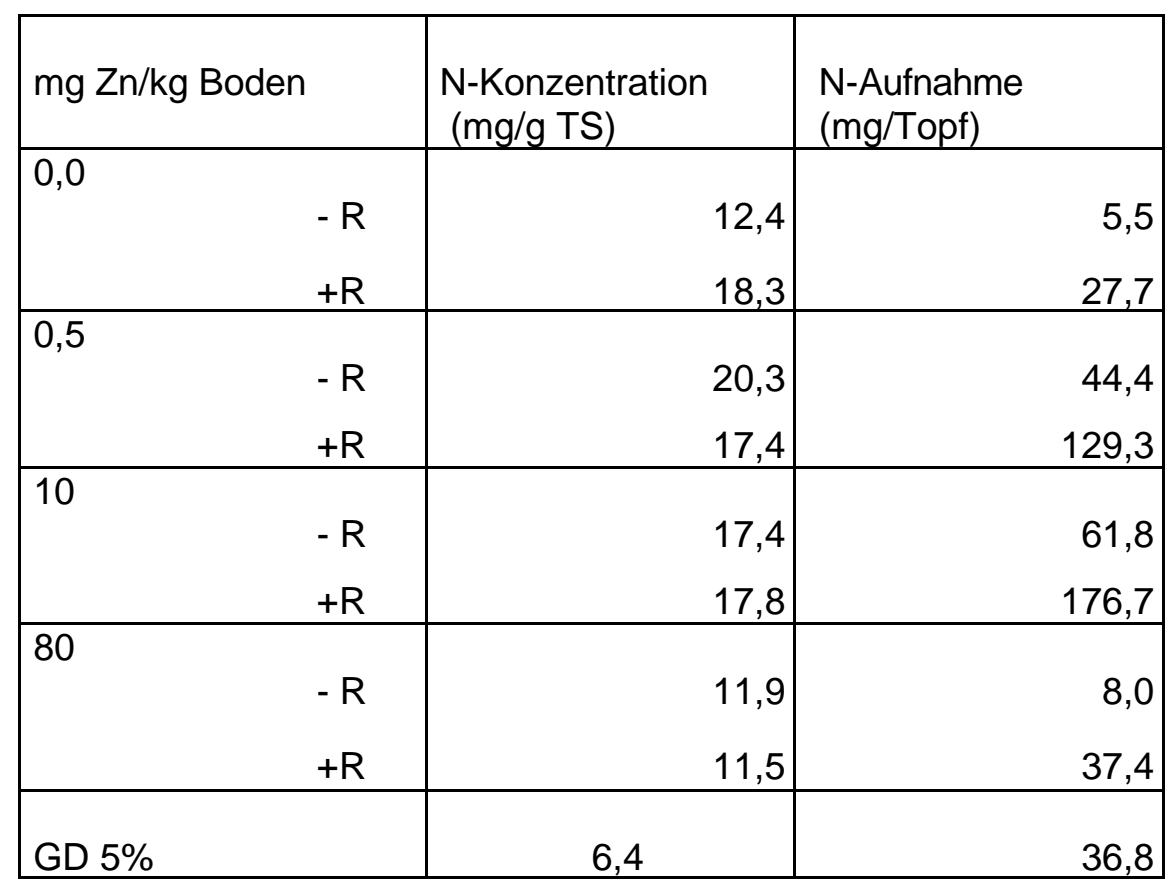

\section{P-Konzentration und -Aufnahme}

Die P-Konzentrationen der unbeimpften Acacia-Pflanzen lagen bei allen Varianten hoch signifikant über denen der beimpften Pflanzen (Tab.100). Insgesamt nahm die PKonzentration durch steigende $\mathrm{Zn}$-Gaben bei den $-\mathrm{R} /$ und $+\mathrm{R} / \mathrm{Pflanzen}$ zunächst ab und dann bei der Zn-Stufe $80 \mathrm{mg} / \mathrm{kg}$ Boden wieder zu. Jedoch war die P-Konzentration bei allen ZnStufen im Spross der $-\mathrm{R} /$ oder $+\mathrm{R} / \mathrm{Pflanzen}$ niedriger als bei der Kontrolle. Sie lag in den $+\mathrm{R} /$ Pflanzen aller Zn-Behandlungen wegen der höheren STG bzw. eines Verdünnungseffektes wesentlich niedriger als in den -R/Pflanzen. Die P-Aufnahme der $-\mathrm{R} /$ und $+\mathrm{R} / \mathrm{-}$ Pflanzen verlief im wesentlichen entsprechend dem Wachstum. Bei den Zn-Stufen 0,5 und 10 ppm stieg die P-Aufnahme im Spross von $+R /$ und $-R / P f l a n z e n$ an, nahm jedoch bei der höchsten Zn-Stufe $80 \mathrm{mg} / \mathrm{kg}$ Boden, besonders bei den -R/Pflanzen deutlich ab. Insgesamt wurde die P-Aufnahme mit steigender Zn-Zugabe bis zu $10 \mathrm{ppm}$ bei den -R/Pflanzen etwas stärker als bei den + R/Pflanzen erhöht, wobei die P-Aufnahme der -R/Pflanzen bei der höchsten Zn-Stufe 80 mg/kg Boden auch stärker als die der +R/Pflanzen absank. 
Tab. 100: Einfluss der Zn-Behandlung (mg/kg Boden) ohne (-R) oder mit Rhizobien (+R) auf die Konzentration (mg/g TS) und Aufnahme (mg/Topf) von P, K, Ca und Mg im Spross von Acacia saligna

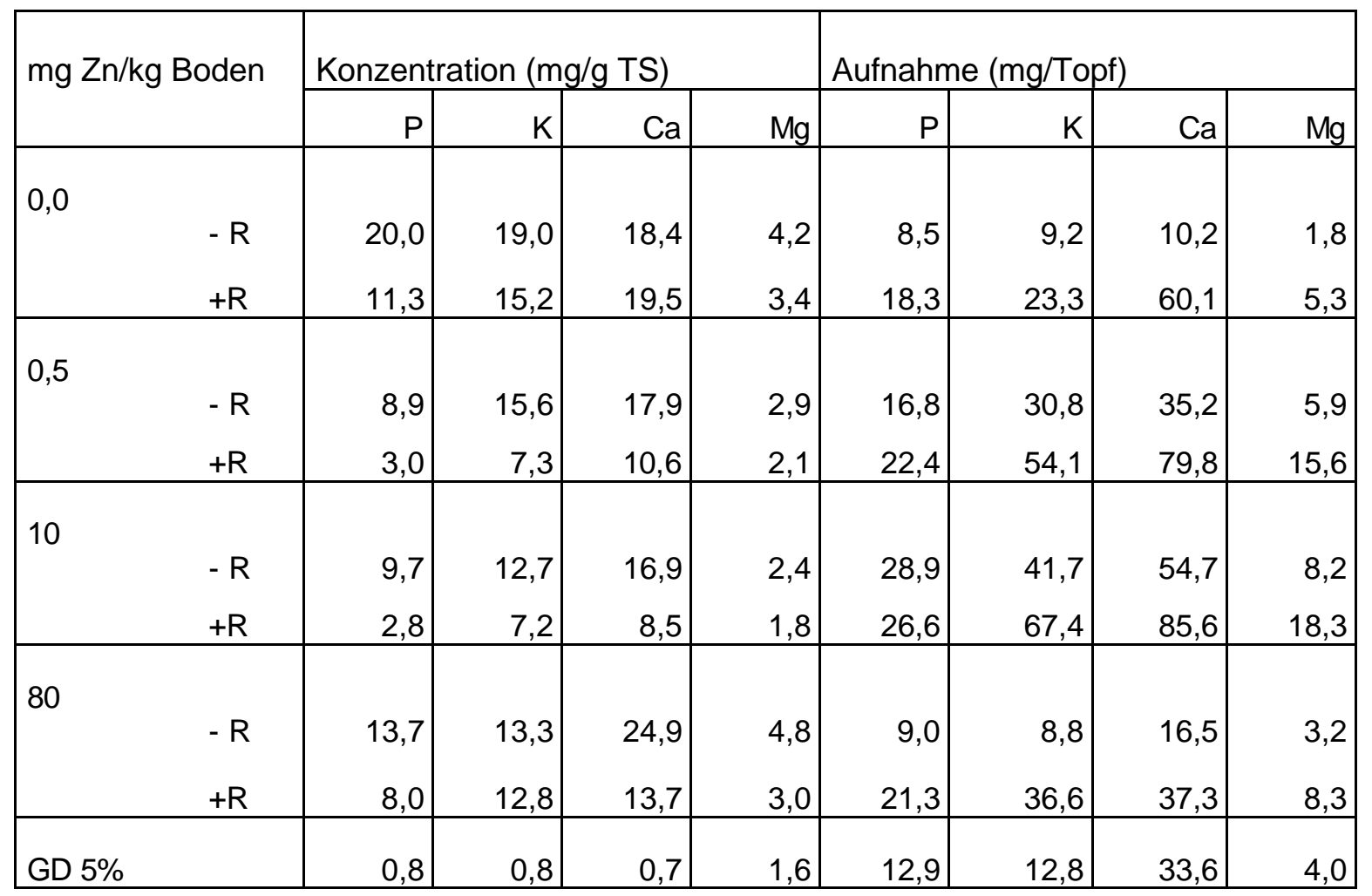

K-Konzentration und -Aufnahme

Insgesamt nahm die K-Konzentration im Spross der $-\mathrm{R} /$ und $+\mathrm{R} /$-Pflanzen durch steigende Zn-Gaben zunächst ab und später bei der höchsten Zn-Stufe 80 ppm wieder zu (Tab.100). Zwischen der Kontrolle und den Zn-Stufen bei den -R/ und +R/Pflanzen waren die Unterschiede signifikant. Die K-Konzentrationen der -R/Pflanzen lagen bei allen Zn-Stufen viel höher als die der + R/Pflanzen wegen der höheren STG bzw. eines Verdünnungseffektes. Die $-\mathrm{R}$ und $+\mathrm{R} /$ Kontrollpflanzen wiesen die höchsten K-Konzentrationen auf. Die KAufnahme im Spross der -R/und +R/Pflanzen zeigte eine ähnliche Tendenz wie das STG. Bei den + R/Pflanzen war die K-Aufnahme wegen der höheren STG 1,6 bis 2,5-mal höher als bei den -R/Pflanzen.

\section{Ca-Konzentration und -Aufnahme}

Die Steigerung der Zn-Zugabe bis $10 \mathrm{mg} / \mathrm{kg}$ Boden führte zu einer Verringerung der CaKonzentration im Spross der -R / und +R/Pflanzen (Tab.100). Die weitere Erhöhung der ZnZugabe auf 80 ppm verursachte eine Steigerung der Ca-Konzentration gegenüber der ZnStufe $10 \mathrm{mg} / \mathrm{kg}$ Boden. Die Verringerung der Ca-Konzentration der +R/Pflanzen war stärker als bei den -R/Pflanzen wegen des bei $\mathrm{P}$ und $\mathrm{K}$ erwähnten Verdünnungseffektes, so dass die Ca-Konzentrationen in den -R/Pflanzen bei allen Zn-Stufen mit Ausnahme der Kontrolle fast 
doppelt so hoch waren wie bei den + R/Pflanzen. Steigende $\mathrm{Zn}$-Stufen führten zu höheren CaAufnahmen, was auf das verbesserte Wachstum zurückzuführen ist. Die Ca-Aufnahme der + R/Pflanzen war bei der Zn-Stufe $10 \mathrm{mg} / \mathrm{kg}$ Boden 1,6fach höher als bei den -R/Pflanzen und 1,4fach höher als in der Kontrolle. Bei der höchsten Zn-Stufe 80 ppm lag sie in den -R/Pflanzen bei einem 1/3 und in den + R/Pflanzen bei $43 \%$ im Vergleich zur Zn-Stufe $10 \mathrm{mg} / \mathrm{kg}$ Boden.

\section{Mg-Konzentration und -Aufnahme}

Bei allen Zn-Stufen lagen die Mg-Konzentrationen der -R/Pflanzen über denen der + R/Pflanzen (Tab.100). Der Unterschied zwischen den beimpften und unbeimpften AcaciaPflanzen war signifikant, allerdings nur bei der höchsten Zn-Stufe $80 \mathrm{mg} / \mathrm{kg}$ Boden. Ansonsten unterschieden sich die anderen Varianten nicht signifikant voneinander. Steigende Zn-Stufen führten aufgrund der höheren STG zu höheren Mg-Aufnahmen. Die Mg-Aufnahme der beimpften Acacia-Pflanzen wurde auf das 2,2 bis 3fache verglichen mit den unbeimpften Pflanzen erhöht.

\section{Zn-Konzentration und -Aufnahme}

Die Zn-Konzentration im Spross der $-\mathrm{R} /$ und $+\mathrm{R} /$ Pflanzen zeigte eine deutliche Steigerung mit zunehmender Zn-Konzentration im Boden (Tab.101). Bei Zn-Zugabe von 10 und besonders von 80 ppm war die Zn-Konzentration in den -R/Pflanzen viel höher als in den $+R /$ Pflanzen. Die -R/Pflanzen wiesen bei der höchsten Zn-Stufe $80 \mathrm{mg} / \mathrm{kg}$ Boden etwa den 15 fachen Wert der Kontrolle auf, wobei die Zn-Konzentration im Spross der -R/Pflanzen derselben Zn-Stufe etwa 3-mal höher als bei den + R/Pflanzen lag. Aufgrund der relativ hohen STG bzw. ZnKonzentrationen lag die Zn-Aufnahme im Spross der -R/ und +R/Pflanzen bei allen Zn-Stufen höher als bei der Kontrolle. Die Zn-Aufnahme der beimpften Acacia-Pflanzen war, außer bei der Kontrolle und Zugabe von 80 ppm Zn, signifikant höher als bei den unbeimpften Pflanzen. 
Tab.101: Einfluss der Zn-Behandlung (mg/kg Boden) ohne (-R) oder mit Rhizobien (+R) auf die Konzentration (mg/g TS) und Aufnahme (mg/Topf) von Zn im Spross von Acacia saligna

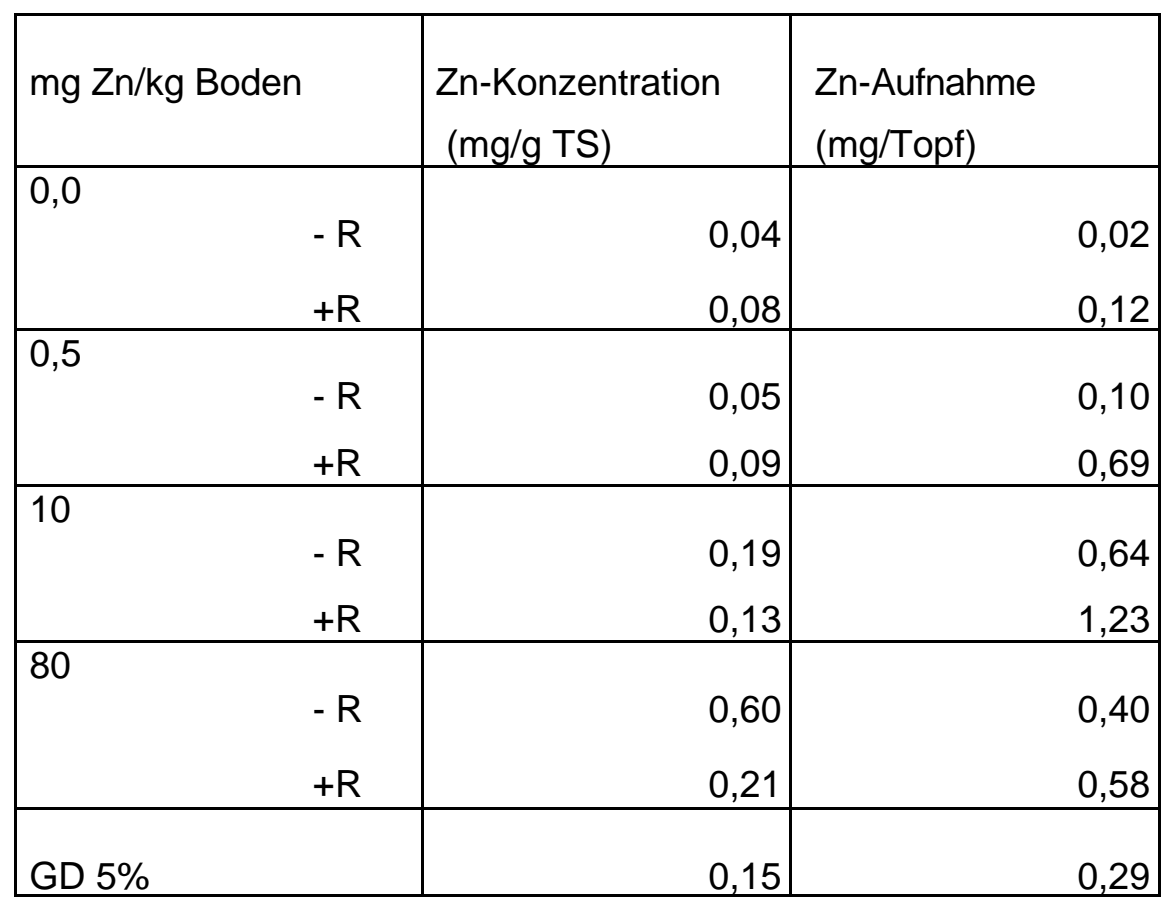

\subsection{Zn-Konzentration und -Aufnahme in der Wurzel}

Die Zn-Konzentrationen und -Aufnahmen in den Wurzeln der - $\mathrm{R} /$ und $+\mathrm{R} /$ Pflanzen ließen ein ähnliches Bild erkennen wie beim Spross; jedoch lagen hier die Werte bei den -R/Pflanzen 4 bis 4,7fach und bei den + R/Pflanzen 2,1 bis 11fach höher als beim Spross (Tab.102. Die ZnAufnahme war bei den $+\mathrm{R} /$ Pflanzen entsprechend dem Wachstum immer höher als bei den -R/Pflanzen, so dass die Zn-Aufnahme in den Wurzeln der - $\mathrm{R} /$ und $+\mathrm{R} / \mathrm{Pflanzen}$ bei den $\mathrm{Zn}$ Stufen 10 und $80 \mathrm{mg} / \mathrm{kg}$ Boden etwa doppelt so hoch war wie im Spross. 
Tab.102: Einfluss der Zn-Behandlung (mg/kg Boden) ohne (-R) oder mit Rhizobien (+R) auf die Konzentration (mg/g TS) und Aufnahme (mg/Topf) von $\mathrm{Zn}$ in der Wurzel von Acacia saligna

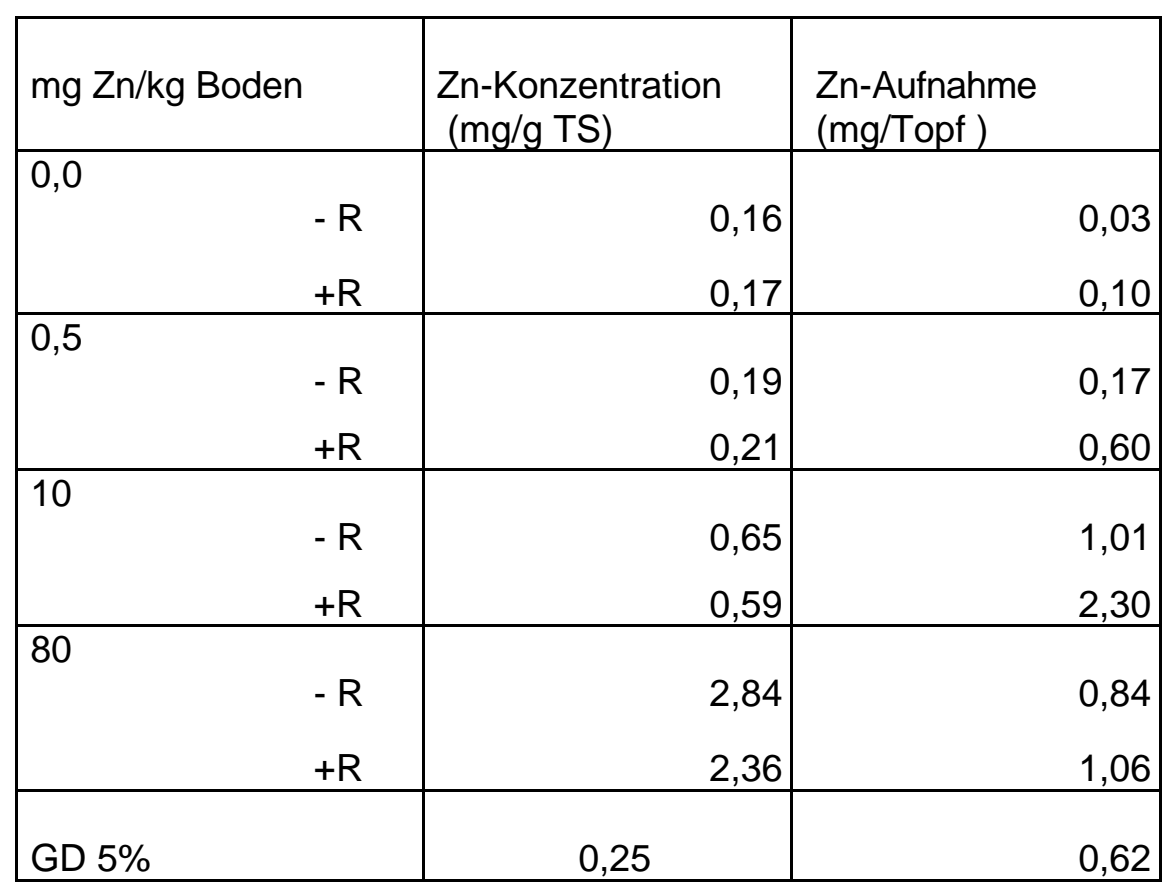

\subsection{Der $\mathrm{pH}-$ Wert des Bodens}

Der pH-Wert sank im Boden am Ende des Versuchs durch die Zn-Behandlung ab (Tab. 103). Insgesamt nahm der $\mathrm{pH}$-Wert im Boden der $+\mathrm{R} /$ und besonders der $-\mathrm{R} /$ Pflanzen durch steigende Zn-Stufen zunächst ab, dann stieg er bei der Zn-Stufe 80 ppm wieder an. Alle pHWerte lagen jedoch niedriger als bei der Kontrolle. Der pH-Wert im Boden der -R/Pflanzen war im allgemeinen etwas höher als im Boden der $+\mathrm{R} /$ Pflanzen.

Tab.103: Einfluss der Zn-Behandlung (mg/kg Boden) ohne (-R) oder mit Rhizobien (+R) auf den $\mathrm{pH}-$ Wert im Boden von Acacia saligna am Ende des Versuches

\begin{tabular}{|c|c|c|c|c|c|}
\hline $\mathrm{Mg} \mathrm{Zn/kg} \mathrm{Boden}$ & 0,0 & 0,5 & 10 & 80 & GD 5\% \\
\hline $\mathrm{PH}-\mathrm{R}$ & 5,8 & 5,6 & 5,3 & 5,4 & \\
\hline $\mathrm{PH}+\mathrm{R}$ & 5,5 & 4,8 & 4,9 & 5,3 & 0,5 \\
\hline
\end{tabular}




\subsubsection{Cd-Behandlung}

\subsection{Sprosstrockengewicht (STG)}

Das STG der $-\mathrm{R} /$ und $+\mathrm{R} /$ Pflanzen stieg bei geringer Cd-Zugabe von $1,25 \mathrm{mg} / \mathrm{kg}$ Boden an und sank bei 5 und $20 \mathrm{mg} / \mathrm{kg}$ Boden ab (Tab.104). Das höchste STG der beimpften und unbeimpften Pflanzen wurde bei der Cd-Stufe 1,25 ppm festgestellt. Die Beimpfung bewirkte in sämtlichen Cd-Behandlungen hoch signifikante Zunahmen des STG verglichen mit den unbeimpften Acacia-Pflanzen; das STG der +R/Pflanzen lag 3- bis 4-mal so hoch wie bei den -R/Pflanzen. Bei der höchsten Cd-Zugabe wurde das STG der Kontrollpflanzen fast wieder erreicht.

Tab.104: Einfluss der Cd-Behandlung (mg/kg Boden) ohne (-R) oder mit Rhizobien (+R) auf das Sprosstrockengewicht (g/Topf) von Acacia saligna

\begin{tabular}{|l|c|c|c|c|l|}
\hline mg Cd/kg Boden & 0,0 & 1,25 & 5 & 20 & GD 5\% \\
\hline $\begin{array}{l}\text { STG g/Topf } \\
-\mathrm{R}\end{array}$ & 0,5 & 1,9 & 1,2 & 0,5 & \\
$\quad \mathrm{R}$ & 1,5 & 7,6 & 5,7 & 2,0 & 1,0 \\
\hline
\end{tabular}

\subsection{Wurzeltrockengewicht (WTG)}

Die WTG zeigten insgesamt eine ähnliche Tendenz wie die STG, lagen jedoch etwas niedriger (Tab.105). Nur bei der Cd-Stufe $1,25 \mathrm{mg} / \mathrm{kg}$ Boden war das WTG der + R/Pflanzen signifikant höher als das der unbeimpften Pflanzen. Das höchste WTG der -R/Pflanzen wurde in der Cd-Stufe $5 \mathrm{mg} / \mathrm{kg}$ Boden ermittelt.

Tab.105: Einfluss der Cd-Behandlung (mg/kg Boden) ohne (-R) oder mit Rhizobien (+R) auf das Wurzeltrockengewicht (g/Topf) von Acacia saligna

\begin{tabular}{|l|c|c|c|c|l|}
\hline mg Cd/kg Boden & 0,0 & 1,25 & 5 & 20 & GD 5\% \\
\hline $\begin{array}{c}\text { WTG g/Topf } \\
-\mathrm{R}\end{array}$ & 0,2 & 0,8 & 1,0 & 0,4 & \\
$+\mathrm{R}$ & 0,6 & 2,7 & 1,7 & 0,8 & 1,0
\end{tabular}




\subsection{Konzentration und Aufnahme von $\mathrm{N}, \mathrm{P}, \mathrm{K}, \mathrm{Ca}, \mathrm{Mg}$ und $\mathrm{Cd}$ im Spross}

\section{N-Konzentration und -Aufnahme}

Der Unterschied in der N-Konzentration zwischen beimpften und unbeimpften AcaciaPflanzen war nur in der Kontrolle signifikant (Tab.106). Im Gegensatz zu den -R/Pflanzen nahm die N-Konzentration der + R/Pflanzen mit steigender Cd-Stufe leicht ab. Die NKonzentrationen der $+\mathrm{R} /$ Pflanzen waren bei allen Cd-Stufen, mit Ausnahme der höchsten Cd-Stufe $20 \mathrm{mg} / \mathrm{kg}$ Boden, etwas höher als bei den $-\mathrm{R} /$ Pflanzen. Die N-Aufnahme der $-R /$ und $+R /$ Pflanzen entsprach weitgehend dem Wachstum. Bei den $+R /$ Pflanzen war die N-Aufnahme 4,5 bis 5fach höher als bei den unbeimpften Pflanzen. Die höchste NAufnahme der $+\mathrm{R} /$ Pflanzen wurde entsprechend dem STG bei der Cd-Stufe $1,25 \mathrm{mg} / \mathrm{kg}$ Boden festgestellt und lag 5 fach höher als in der Kontrolle. Die -R/Pflanzen erreichten bei der Cd-Stufe 1,25 ppm ihre höchste N-Aufnahme (5,5 mal höher als bei den Kontrollpflanzen).

Tab.106: Einfluss der Cd-Behandlung (mg/kg Boden) ohne (-R) oder mit Rhizobien (+R) auf die Konzentration (mg/g TS) und Aufnahme (mg/Topf) von $\mathrm{N}$ im Spross von Acacia saligna

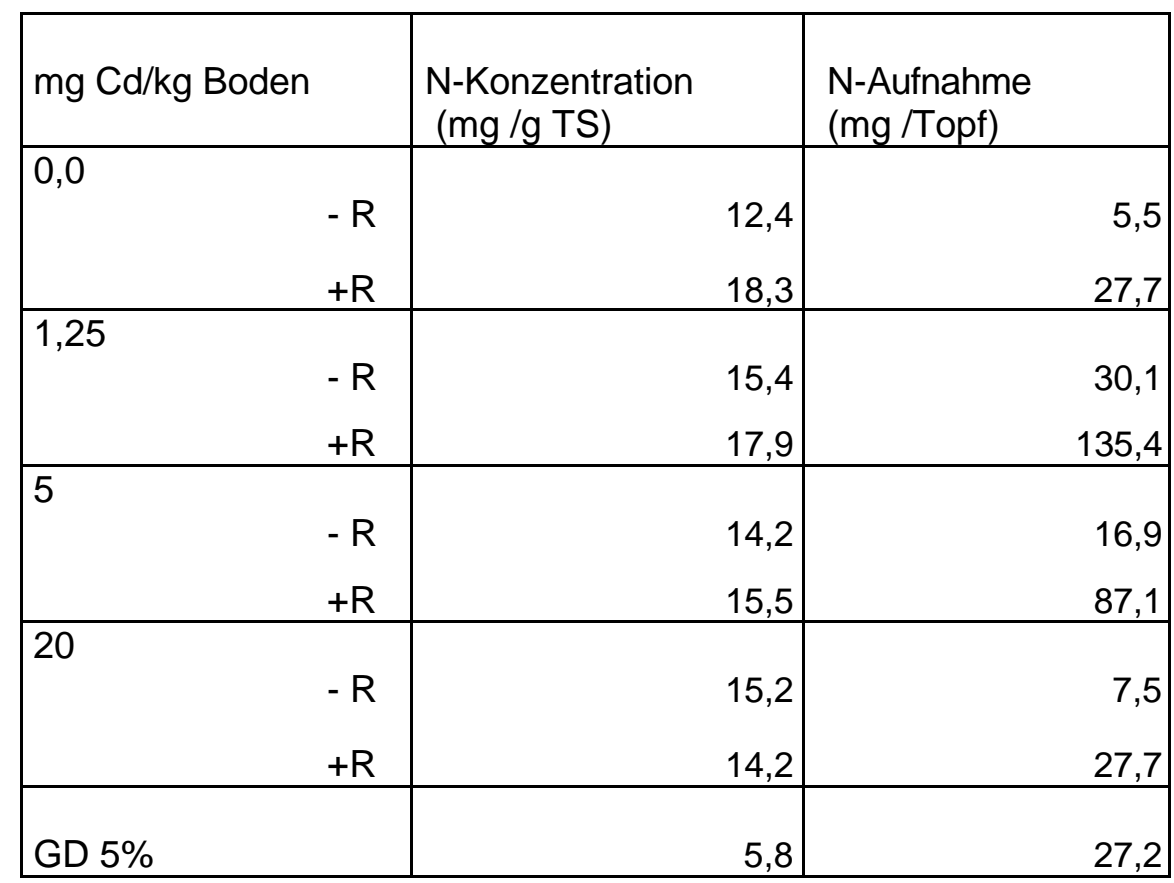

P-Konzentration und -Aufnahme

Die P-Konzentration nahm im Spross der -R/ und +R/Pflanzen mit zunehmender CdZugabe bis zu 1,25 mg/kg Boden gegenüber der Kontrolle ab, danach stieg sie wieder an (Tab.107). Alle Werte lagen jedoch niedriger als bei der Kontrolle. Zwischen den -R/ und + R/Pflanzen wurden bei allen Cd-Stufen mit Ausnahme der Kontrolle und 1,25 ppm Cd 
keine nennenswerten Unterschiede in der P-Konzentration festgestellt, während die PKonzentration der -R/Pflanzen in der Kontrolle 1,8fach höher lag als bei den +R/Pflanzen. Die P-Aufnahme entsprach im wesentlichen dem Wachstum. Die P-Aufnahme der-R/ und $+\mathrm{R} /$ Pflanzen stieg bis zur Cd-Stufe $5 \mathrm{mg} / \mathrm{kg}$ Boden an. Die höchste Cd-Zugabe verringerte jedoch die P-Aufnahme der -R/ und +R/Pflanzen deutlich. Der hohe GD-Wert erschwert den Vergleich zwischen den verschiedenen Behandlungen.

Tab.107: Einfluss der Cd-Behandlung (mg/kg Boden) ohne (-R) oder mit Rhizobien (+R) auf die Konzentration (mg/g TS) und Aufnahme (mg/Topf) von P, K, Ca und Mg im Spross von Acacia saligna

\begin{tabular}{|c|c|c|c|c|c|c|c|c|}
\hline \multirow[t]{2}{*}{$\mathrm{mg} \mathrm{Cd} / \mathrm{kg}$ Boden } & \multicolumn{4}{|c|}{ Konzentration (mg/g TS) } & \multicolumn{4}{|c|}{ Aufnahme (mg/Topf) } \\
\hline & $\mathrm{P}$ & $\mathrm{K}$ & $\mathrm{Ca}$ & $\mathrm{Mg}$ & $\mathrm{P}$ & $\mathrm{K}$ & $\mathrm{Ca}$ & $\mathrm{Mg}$ \\
\hline 0,0 & 20,0 & 19,0 & 18,4 & 4,2 & 8,5 & 9,2 & 10,2 & 1,8 \\
\hline$+\mathrm{R}$ & 11,3 & 15,2 & 19,5 & 3,4 & 18,3 & 23,3 & 30,7 & 5,3 \\
\hline 1,25 & 6,8 & 14,4 & 17,4 & 3,2 & 12,5 & 26,8 & 31,6 & 5,9 \\
\hline$+\mathrm{R}$ & 5,5 & 9,5 & 11,4 & 2,9 & 40,7 & 71,6 & 85,4 & 21,8 \\
\hline 5 & 10,9 & 15,7 & 17,1 & 3,1 & 11,6 & 17,3 & 19,1 & 3,5 \\
\hline$+\mathrm{R}$ & 11,4 & 14,7 & 15,7 & 3,5 & 65,8 & 84,8 & 94,7 & 20,3 \\
\hline 20 & 10,8 & 12,4 & 14,1 & 2,7 & 5,1 & 6,2 & 6,4 & 1,3 \\
\hline$+\mathrm{R}$ & 11,6 & 15,4 & 16,1 & 2,5 & 22,8 & 31,5 & 32,7 & 5,0 \\
\hline GD 5\% & 0,9 & 0,8 & 1,2 & 1,7 & 39,5 & 27,1 & 49,0 & 8,7 \\
\hline
\end{tabular}

\section{K-Konzentration und -Aufnahme}

Die Steigerung der Cd-Zugabe führte, mit Ausnahme der höchsten Cd-Stufe, zu einer Verringerung der K-Konzentration im Spross der $-\mathrm{R} /$ und $+\mathrm{R} /$ Pflanzen gegenüber der Kontrolle (Tab. 107). Die K-Konzentration der -R/Pflanzen lag bei allen Cd-Stufen, mit Ausnahme der höchsten Cd-Stufe $20 \mathrm{mg} / \mathrm{kg}$ Boden, signifikant höher als bei den beimpften Pflanzen. Die K-Aufnahme der -R/und +R/Pflanzen verlief im wesentlichen entsprechend dem Wachstum. Bei den beimpften Acacia-Pflanzen war die K-Aufnahme wesentlich höher als bei den unbeimpften Pflanzen. Im allgemeinen wurde das Wachstum durch die Cd-Zugabe stärker gefördert als die K-Aufnahme. 


\section{Ca-Konzentration und -Aufnahme}

Wie beim Kalium, führte die zunehmende Cd-Konzentration im Boden zu einer Verringerung der Ca-Konzentration im Spross der beimpften und unbeimpften Pflanzen (Tab.107). Alle Werte der Ca-Konzentrationen im Spross von den -R/ und +R/Pflanzen lagen niedriger als in der Kontrolle. Zwischen den $-\mathrm{R} /$ und $+\mathrm{R} / \mathrm{Pflanzen}$ waren signifikante Unterschiede der Ca-Konzentration bei allen Cd-Stufen mit Ausnahme bei der Kontrolle festzustellen. Die Ca-Aufnahme der $-\mathrm{R} /$ und $+\mathrm{R} /$ Pflanzen entsprach dem Wachstum. Die Ca-Aufnahme war bei den beimpften Acacia-Pflanzen mehrfach höher als bei den unbeimpften Pflanzen.

\section{Mg-Konzentration und -Aufnahme}

Zwischen den $-\mathrm{R} /$ und $+\mathrm{R} /$ Pflanzen waren keine signifikanten Unterschiede in der $\mathrm{Mg}$ Konzentration festzustellen (Tab.107). Die Mg-Aufnahme im Spross der -R/ und der + R/Pflanzen entsprach im wesentlichen dem Wachstum, d.h. sie wurde weder durch die Beimpfung noch die Cd-Zugabe beeinflusst.

\section{Cd-Konzentration und -Aufnahme}

Die unbeimpften Acacia-Pflanzen wiesen höhere Cd-Konzentration als die beimpften Pflanzen auf (Tab.108). Die Cd-Konzentrationen stiegen im Spross der -R/ und +R/Pflanzen besonders bei den höchsten Cd-Stufen stark an. Aufgrund der relativ hohen TG bzw. hohen Cd-Konzentrationen lag die Cd-Aufnahme im Spross der - R/ und $+\mathrm{R} /$ Pflanzen bei allen Cd-Stufen höher als in der Kontrolle. Die Cd-Aufnahme der beimpften Acacia-Pflanzen war entsprechend dem Wachstum höher als die der unbeimpften Pflanzen. 
Tab.108: Einfluss der Cd-Behandlung (mg/kg Boden) ohne (-R) oder mit Rhizobien (+R) auf die Konzentration (mg/g TS) und Aufnahme (mg/Topf) von Cd im Spross von Acacia saligna

\begin{tabular}{|lc|r|l|}
\hline mg Cd/kg Boden & & $\begin{array}{l}\text { Cd-Konzentration } \\
\text { (mg/g TS) }\end{array}$ & \multicolumn{2}{|l|}{$\begin{array}{l}\text { Cd-Aufnahme } \\
\text { (mg/Topf })\end{array}$} \\
\hline 0,0 & $-\mathrm{R}$ & 0,00 & 0,00 \\
& $+\mathrm{R}$ & 0,00 & 0,00 \\
\hline 1,25 & $-\mathrm{R}$ & 0,03 & 0,06 \\
& $+\mathrm{R}$ & 0,01 & 0,08 \\
\hline 5 & $-\mathrm{R}$ & 0,12 & 0,13 \\
& $+\mathrm{R}$ & 0,11 & 0,69 \\
\hline 20 & $-\mathrm{R}$ & 0,33 & 0,16 \\
& $+\mathrm{R}$ & 0,21 & 0,45 \\
\hline GD 5\% & & 0,06 & 0,29 \\
\hline
\end{tabular}

\subsection{Cd-Konzentration und -Aufnahme in der Wurzel}

Ein in der Tendenz sehr ähnliches Bild wie beim STG hinsichtlich der Cd-Akkumulation war auch beim WTG zu sehen (Tab.109), allerdings mit Werten, die vielfach höher lagen als im Spross. Ebenso wie bei den $+\mathrm{R} /$ Pflanzen stiegen die Cd-Konzentrationen der unbeimpften Acacia-Pflanzen mit steigender Cd-Stufe an; bei den -R/Pflanzen aber viel stärker als bei den beimpften Pflanzen. Wegen der hohen Cd-Konzentration in den -R/Pflanzen bei allen Cd-Stufen lagen die Cd-Aufnahmen hier allgemein viel höher als in den $+\mathrm{R} /$ Pflanzen, obwohl das Wachstum der $+\mathrm{R} /$ Pflanzen besser als das der -R/Pflanzen war. Die unbeimpften Acacia-Pflanzen nahmen ungefähr doppelt so viel $\mathrm{Cd}$ wie die beimpften Pflanzen auf, besonders bei den Cd-Stufen 5 und $20 \mathrm{mg} / \mathrm{kg}$ Boden. Hier scheint die Beimpfung die Cd-Aufnahme zu behindern. 
Tab.109: Einfluss der Cd-Behandlung (mg/kg Boden) ohne (-R) oder mit Rhizobien (+R) auf die Konzentration (mg/g TS) und Aufnahme (mg/Topf) von Cd in der Wurzel von Acacia saligna

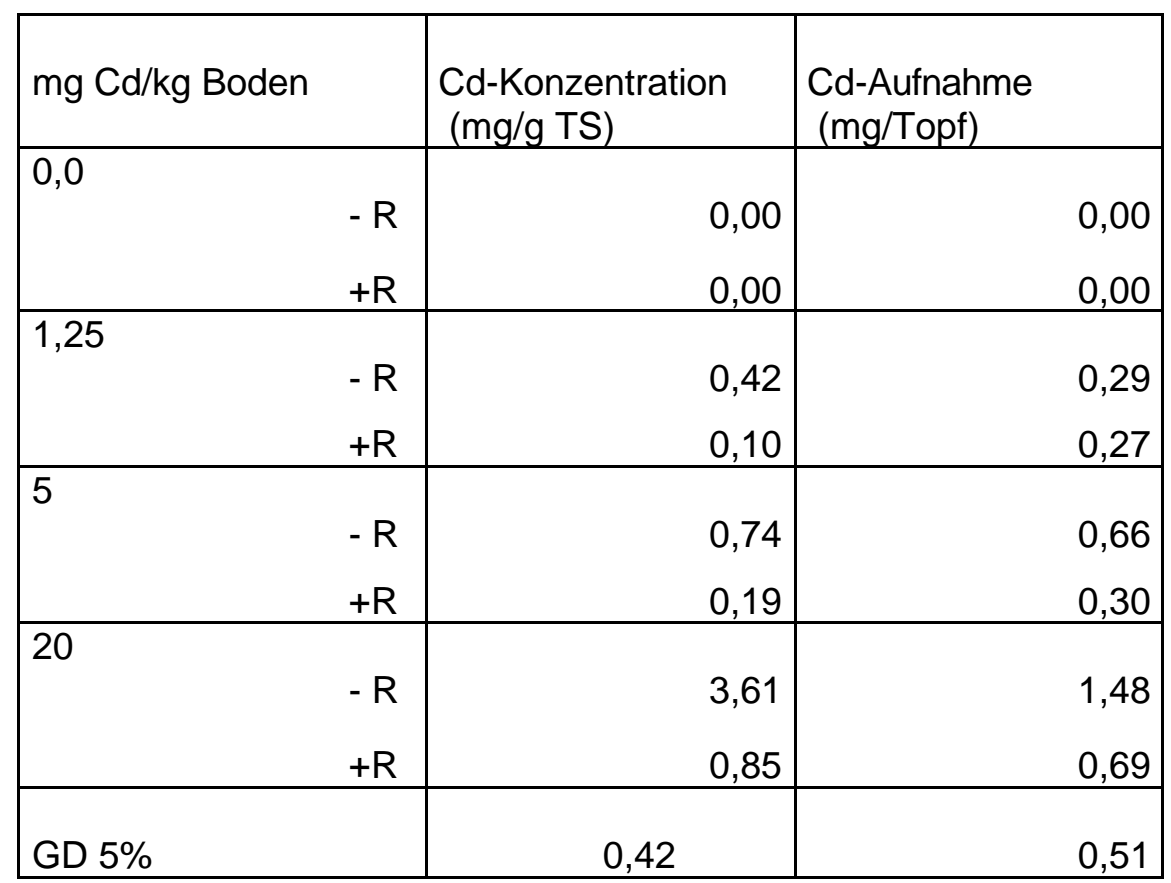

\subsection{Der $\mathrm{pH}-$ Wert des Bodens}

Der $\mathrm{pH}$-Wert im Boden bei den $-\mathrm{R} /$ und $+\mathrm{R} / \mathrm{Pflanzen}$ aller Cd-Behandlungen wies allgemein ähnliche Tendenzen wie in den Zn-Behandlungen auf (Tab.110). Die Werte lagen jedoch hier etwas höher. Das Ausgangs-Boden-pH von 7 sank bis auf 5,4 am Ende der Vegetationpriode ab.

Tab. 110: Einfluss der Cd-Behandlung (mg/kg Boden) ohne (-R) oder mit Rhizobien (+R) auf den pH-Wert im Boden von Acacia saligna am Ende des Versuches

\begin{tabular}{|l|c|c|c|c|l|}
\hline $\mathrm{mg} \mathrm{Cd} / \mathrm{kg}$ Boden & 0,0 & 1,25 & 5 & 20 & GD 5\% \\
\cline { 1 - 5 } $\mathrm{pH}-\mathrm{R}$ & 5,8 & 5,5 & 5,5 & 5,7 & \\
\cline { 1 - 4 }$+\mathrm{pH}$ & 5,5 & 5,4 & 5,4 & 5,5 & 0,6 \\
\hline
\end{tabular}




\subsubsection{Pb-Behandlung}

\subsection{Sprosstrockengewicht (STG)}

Mit steigender Pb-Zugabe nahm das STG der -R/ und +R/Pflanzen stark zu (Tab.111). Die Beimpfung erhöhte das Wachstum der Acacia-Pflanzen bei sämtlichen Pb-Stufen hoch signifikant; die STG der +R/Pflanzen waren zwei- bis dreimal höher als die der -R/Pflanzen. Das stärkste Wachstum der +R/Pflanzen wurde bei der Pb-Stufe $10 \mathrm{mg} / \mathrm{kg}$ Boden ermittelt, dieser Wert unterschied sich hoch signifikant von den anderen $\mathrm{Pb}$-Stufen. Das höchste STG der -R/Pflanzen wurde auch bei beiden Pb-Stufen 5 und 10 ppm ermittelt. Die Schädigungen durch steigende Pb-Konzentrationen im Boden waren bei den + R/Pflanzen schwächer als bei den unbeimpften Pflanzen. Bemerkenswert waren die hohen STG der beimpften und unbeimpften Pflanzen bei der höchsten Pb-Zugabe $80 \mathrm{mg} / \mathrm{kg}$ Boden im Vergleich zur Kontrolle.

Tab.111: Einfluss der Pb-Behandlung (mg/kg Boden) ohne (-R) oder mit Rhizobien auf das Sprosstrockengewicht (g/Topf) von Acacia saligna

\begin{tabular}{|l|c|c|c|c|l|}
\hline mg Pb/kg Boden & 0,0 & 2,5 & 10 & 80 & GD 5\% \\
\hline $\begin{array}{l}\text { STG g/Topf } \\
-\mathrm{R}\end{array}$ & 0,5 & 4,6 & 4,9 & 2,4 & \\
$\quad \mathrm{R}$ & 1,5 & 5,7 & 9,9 & 6,3 & 2,2 \\
\hline
\end{tabular}

\subsection{Wurzeltrockengewicht (WTG)}

Das WTG zeigte eine sehr ähnliche Tendenz wie das STG ( Tab.112), allerdings mit deutlich niedrigeren Werten.

Tab.112: Einfluss der Pb-Behandlung (mg/kg Boden) ohne (-R) oder mit Rhizobien (+R) auf das Wurzeltrockengewicht (g/Topf) von Acacia saligna

\begin{tabular}{|l|c|c|c|c|l|}
\hline mg Pb/kg Boden & 0,0 & 2,5 & 10 & 80 & GD 5\% \\
\hline $\begin{array}{l}\text { WTG g/Topf } \\
-\mathrm{R}\end{array}$ & 0,2 & 1,3 & 2,1 & 0,8 & \\
$\quad \mathrm{R}$ & 0,6 & 2,1 & 4,4 & 1,9 & 1,3 \\
\hline
\end{tabular}


5.3.1.3.3 Konzentration und Aufnahme von $\mathrm{N}, \mathrm{P}, \mathrm{K}, \mathrm{Ca}, \mathrm{Mg}$ und $\mathrm{Pb}$ im Spross

$\mathrm{N}$-Konzentration und -Aufnahme

Die N-Konzentration stieg bei den unbeimpften Pflanzen mit steigenden $\mathrm{Pb}-\mathrm{Gaben}$ an (Tab.113). Diese Steigerung lag jedoch nur bei der Pb-Stufe $10 \mathrm{mg} / \mathrm{kg}$ Boden signifikant über der Kontrolle. Die N-Konzentrationen im Spross der beimpften Pflanzen wiesen keine signifikanten Unterschiede auf. Zwischen den $-\mathrm{R} /$ und $+\mathrm{R} / \mathrm{Pflanzen}$ ergaben sich ebenfalls keine signifikanten Unterschiede. Steigende $\mathrm{Pb}$-Zugaben führten zu deutlich höheren $\mathrm{N}$ Aufnahmen, was auf das verbesserte Wachstum zurückzuführen ist. Die höchste NAufnahme der + R/Pflanzen wurde bei der Pb-Stufe $10 \mathrm{mg} / \mathrm{kg}$ Boden bestimmt und lag 7 fach höher als in der Kontrolle. Bei derselben $\mathrm{Pb}$-Stufe wurde auch die höchste NAufnahme der -R/Pflanzen festgestellt. Sie lag 18 fach höher als in der Kontrolle. Die NAufnahme der beimpften Acacia-Pflanzen war allgemein etwa 2 bis 5 mal so hoch wie bei den unbeimpften Pflanzen.

Tab.113: Einfluss der Pb-Behandlung (mg/kg Boden) ohne (-R) oder mit Rhizobien (+R) auf die Konzentration (mg/g TS) und Aufnahme (mg/Topf) von $\mathrm{N}$ im Spross von Acacia saligna

\begin{tabular}{|lr|r|r|}
\hline mg Pb/kg Boden & & $\begin{array}{c}\text { N-Konzentration } \\
\text { (mg/g TS) }\end{array}$ & \multicolumn{2}{|c|}{$\begin{array}{l}\text { N-Aufnahme } \\
\text { (mg/Topf) }\end{array}$} \\
\hline 0,0 & $-\mathrm{R}$ & 12,4 & 5,5 \\
& $+\mathrm{R}$ & 18,3 & 27,7 \\
\hline 2,5 & $-\mathrm{R}$ & 16,7 & 80,5 \\
& $+\mathrm{R}$ & 19,9 & 116,0 \\
\hline 10 & $-\mathrm{R}$ & 19,5 & 97,9 \\
& $+\mathrm{R}$ & 18,0 & 181,5 \\
\hline 80 & $-\mathrm{R}$ & 17,9 & 45,1 \\
& $+\mathrm{R}$ & 14,2 & 89,1 \\
\hline \multirow{2}{*}{ GD 5\% } & & 6,8 & 58,0 \\
\hline
\end{tabular}

\section{P-Konzentration und -Aufnahme}

Die P-Konzentration im Spross der $-\mathrm{R} /$ und $+\mathrm{R} /$ Pflanzen nahm deutlich mit zunehmenden $\mathrm{Pb}$-Gaben ab (Tab.114). Die Unterschiede der P-Konzentration zwischen den beimpften und unbeimpften Acacia-Pflanzen waren bei den $\mathrm{Pb}$-Stufen mit Ausnahme $10 \mathrm{ppm} \mathrm{Pb}$ signifikant. Die höchste P-Konzentration in den $-\mathrm{R} /$ und $+\mathrm{R} /$ Pflanzen wurde bei den Kontrollen ermittelt. Die P-Konzentrationen der -R/Pflanzen, mit Ausnahme der Pb-Stufe 
$10 \mathrm{mg} / \mathrm{kg}$ Boden, war in etwa doppelt so hoch wie in den $+\mathrm{R} /$ Pflanzen. Die P-Aufnahme der $-\mathrm{R} /$ und $+\mathrm{R} /$ Pflanzen entsprach nicht immer dem Wachstum. Die P-Aufnahme der -R/Pflanzen lag aufgrund der relativ hohen P-Konzentration bei den $\mathrm{Pb}$-Stufen 2,5 und $80 \mathrm{mg} / \mathrm{kg}$ Boden höher als in den $+\mathrm{R} /$ Pflanzen. Generell waren alle P-Aufnahmen der -R/ und $+\mathrm{R} /$ Pflanzen höher als in den Kontrollen. Die höchste P-Aufnahme der $+\mathrm{R} /$ Pflanzen wurde entsprechend dem Wachstum bei der Pb-Stufe $10 \mathrm{mg} / \mathrm{kg}$ Boden festgestellt. Sie lag etwa doppelt so hoch wie bei den -R/Pflanzen und 3-mal höher als in der Kontrolle.

Tab.114: Einfluss der Pb-Behandlung (mg/kg Boden) ohne (-R) oder mit Rhizobien (+R) auf die Konzentration (mg/g TS) und Aufnahme (mg/Topf) von P, K, Ca und Mg im Spross von Acacia saligna

\begin{tabular}{|c|c|c|c|c|c|c|c|c|c|}
\hline \multirow{2}{*}{\multicolumn{2}{|c|}{ mg Pb/kg Boden }} & \multicolumn{4}{|c|}{ Konzentration (mg/g TS) } & \multicolumn{4}{|c|}{ Aufnahme (mg/Topf) } \\
\hline & & $P$ & K & $\mathrm{Ca}$ & $\mathrm{Mg}$ & $P$ & K & $\mathrm{Ca}$ & $\mathrm{Mg}$ \\
\hline \multirow[t]{2}{*}{0,0} & $-R$ & 20,0 & 19,0 & 18,4 & 4,2 & 8,5 & 9,2 & 10,2 & 1,8 \\
\hline & $+\mathrm{R}$ & 11,3 & 15,2 & 19,5 & 3,4 & 18,3 & 23,3 & 30,7 & 5,3 \\
\hline \multirow[t]{2}{*}{2,5} & $-R$ & 8,9 & 13,9 & 17,3 & 3,7 & 36,9 & 60,4 & 75,3 & 16,3 \\
\hline & $+\mathrm{R}$ & 3,9 & 11,0 & 11,1 & 2,8 & 20,8 & 61,5 & 60,9 & 15,8 \\
\hline \multirow[t]{2}{*}{10} & $-R$ & 5,9 & 9,8 & 13,8 & 3,0 & 26,2 & 46,7 & 65,0 & 13,9 \\
\hline & $+\mathrm{R}$ & 5,7 & 8,7 & 11,9 & 2,4 & 54,0 & 83,2 & 112,5 & 23,1 \\
\hline \multirow[t]{2}{*}{80} & $-R$ & 9,4 & 12,6 & 18,4 & 3,9 & 21,1 & 31,0 & 43,9 & 9,2 \\
\hline & $+\mathrm{R}$ & 3,8 & 8,6 & 11,3 & 2,4 & 18,8 & 46,8 & 61,1 & 13,2 \\
\hline GD 5\% & & 0,7 & 0,8 & 1,1 & 1,7 & 38,2 & 30,4 & 34,9 & 6,6 \\
\hline
\end{tabular}

K-Konzentration und -Aufnahme

Die unbeimpften Acacia-Pflanzen wiesen signifikant höhere K-Konzentrationen als die beimpften Pflanzen auf (Tab.114). Die K-Konzentrationen der + R/Pflanzen nahmen bei allen $\mathrm{Pb}$-Stufen $\mathrm{ab}$. Alle K-Konzentrationen der $-\mathrm{R} /$ und $+\mathrm{R} / \mathrm{Pflanzen}$ lagen niedriger als in den Kontrollen und weisen auf einen Verdünnungseffekt hin. Die K-Aufnahme der -R/ und $+\mathrm{R} /$ Pflanzen entsprach im wesentlichen dem Wachstum. Die K-Aufnahme war bei den beimpften Acacia-Pflanzen bei allen Pb-Stufen höher als bei den unbeimpften Pflanzen. 


\section{Ca-Konzentration und -Aufnahme}

Die Ca-Konzentration war im Spross der -R/Pflanzen bei allen Pb-Stufen, mit Ausnahme der Kontrolle, deutlich höher als bei den +R/Pflanzen (Tab.114). Die Ca-Aufnahme im Spross der $-\mathrm{R} /$ und $+\mathrm{R} /$ Pflanzen verlief meistens entsprechend dem Wachstum. Die CaAufnahme war bei den + R/Pflanzen höher als bei den -R/Pflanzen, jedoch lag die CaAufnahme der -R/Pflanzen aufgrund höherer $\mathrm{Ca}-$ Konzentration bei der Pb-Stufe 2,5 ppm höher als die der + R/Pflanzen.

\section{Mg-Konzentration und -Aufnahme}

Generell war die Mg-Konzentration bei den unbeimpften Pflanzen etwas höher als bei den beimpften Pflanzen (Tab.114). Steigende Pb-Gaben führten aufgrund der hohen STG zu höheren Mg-Aufnahmen der $-\mathrm{R} /$ und $+\mathrm{R} /$ Pflanzen. Die Mg-Aufnahme war bei den beimpften Pflanzen meistens höher als bei den unbeimpften Pflanzen, die Mg-Aufnahme der -R/Pflanzen war wegen hoher Mg-Konzentration bei der Pb-Stufe 2,5 mg/kg Boden etwas höher als die der $+\mathrm{R} /$ Pflanzen.

$\mathrm{Pb}$-Konzentration und -Aufnahme

Im Spross der $-\mathrm{R} /$ und $+\mathrm{R} / \mathrm{Pflanzen}$ zeigte sich eine starke Erhöhung der Pb-Konzentration mit steigender $\mathrm{Pb}$-Stufe, sie lag im Spross der -R/Pflanzen bei der höchsten $\mathrm{Pb}$-Stufe $80 \mathrm{mg} / \mathrm{kg}$ Boden etwa 24fach höher als bei der Kontrolle (Tab.115). Hingegen stieg die PbKonzentration der $+\mathrm{R} /$ Pflanzen bei derselben $\mathrm{Pb}$-Stufe nur etwa 7 fach gegenüber der Kontrolle an. Die Pb-Aufnahme der -R/Pflanzen lag etwas höher als die der $+\mathrm{R} /$ Pflanzen, obwohl die $+\mathrm{R} / \mathrm{Pflanzen}$ höhere STG aufwiesen. Im allgemeinen wurde $\mathrm{Pb}$ durch die $\mathrm{Pb}$ Gaben wesentlich stärker und unproportional zur Wachstumsverbesserung aufgenommen. 
Tab.115: Einfluss der Pb-Behandlung (mg/kg Boden) ohne (-R) oder mit Rhizobien (+R) auf die Konzentration (mg/g TS) und Aufnahme (mg/Topf) von $\mathrm{Pb}$ im Spross von Acacia saligna

\begin{tabular}{|lc|r|r|}
\hline mg Pb/kg Boden & & $\begin{array}{c}\text { Pb-Konzentration } \\
\text { (mg/g TS) }\end{array}$ & $\begin{array}{l}\text { Pb-Aufnahme } \\
\text { (mg/Topf) }\end{array}$ \\
\hline 0,0 & $-\mathrm{R}$ & 0,006 & 0,003 \\
& $+\mathrm{R}$ & 0,007 & 0,010 \\
\hline 2,5 & $-\mathrm{R}$ & 0,016 & 0,074 \\
& $+\mathrm{R}$ & 0,012 & 0,069 \\
\hline 10 & $-\mathrm{R}$ & 0,035 & 0,175 \\
& $+\mathrm{R}$ & 0,015 & 0,150 \\
\hline 80 & $-\mathrm{R}$ & 0,143 & 0,336 \\
& $+\mathrm{R}$ & 0,052 & 0,300 \\
\hline \multirow{2}{*}{ GD 5\% } & & 0,030 & 0,081 \\
\hline
\end{tabular}

\subsection{Pb-Konzentration und -Aufnahme in der Wurzel}

Obwohl die Pb-Konzentrationen bzw. -Aufnahmen in den Wurzeln eine ähnliche Tendenz wie in den Sprossen aufwiesen, lagen die Werte der Wurzeln mehrfach höher als in den Sprossen (Tab.116). Anders als in den Sprossen wurde darüber hinaus die $\mathrm{Pb}$-Konzentration bzw. -Aufnahme in den Wurzeln der + R/Pflanzen noch drastischer erhöht als in den -R/Pflanzen. 
Tab.116: Einfluss der Pb-Behandlung (mg/kg Boden) ohne (-R) oder mit Rhizobien (+R) auf die Konzentration (mg/g TS) und Aufnahme (mg/Topf) von $\mathrm{Pb}$ in der Wurzel von Acacia saligna

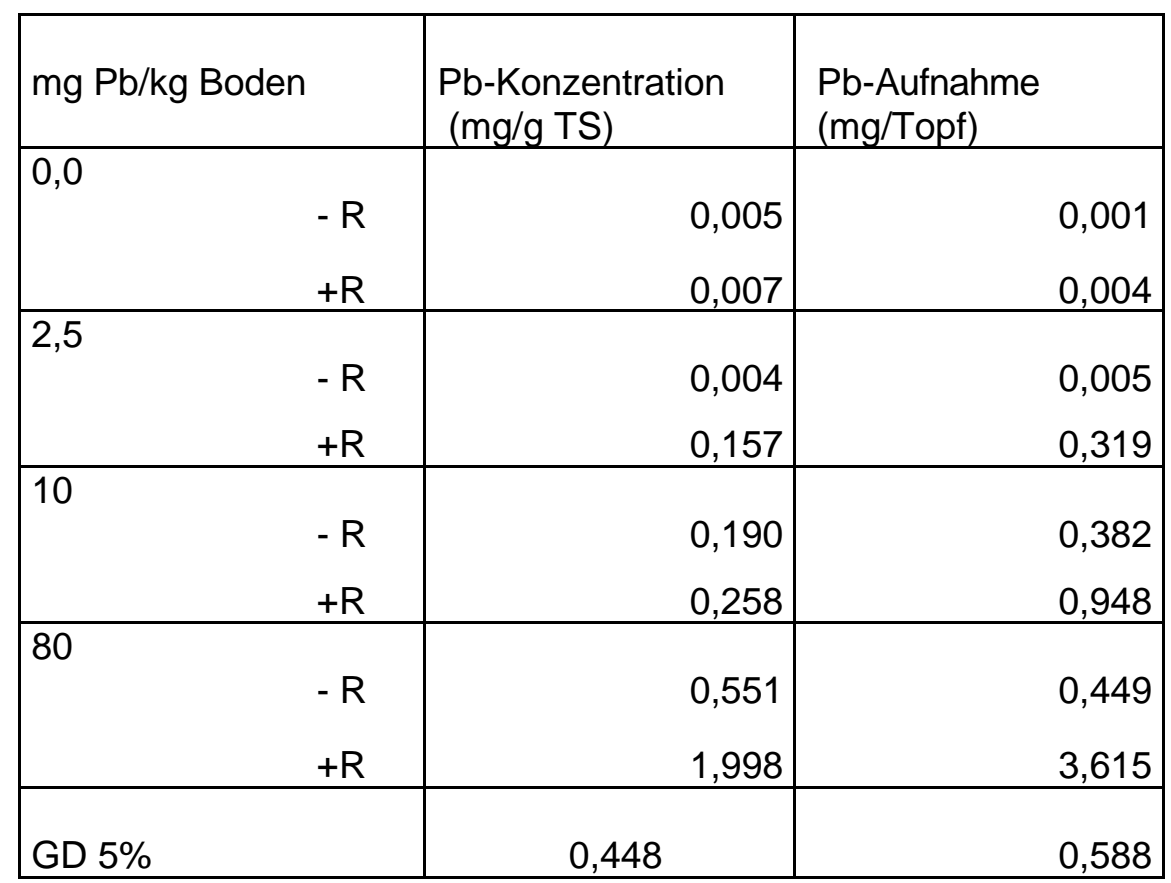

\subsection{Der $\mathrm{pH}-$ Wert des Bodens}

Wie bei den anderen beiden Schwermetallen $\mathrm{Zn}$ und $\mathrm{Cd}$ zeigte sich ebenfalls beim $\mathrm{Pb}$, dass die $\mathrm{pH}$-Werte des Bodens bei allen $-\mathrm{R} /$ und $+\mathrm{R} / \mathrm{Pflanzen}$ mit steigender Pb-Zugabe abnahmen (Tab.117). Jedoch waren die Werte bei den $-\mathrm{R} /$ und $+\mathrm{R} / \mathrm{Pflanzen}$ aller $\mathrm{Pb}-$ Behandlungen geringfügig niedriger als bei den Behandlungen mit $\mathrm{Zn}$ oder Cd. Das Ausgangs-Boden-pH von $7 \mathrm{zu}$ Beginn des Versuches sank sowohl bei den -R/Pflanzen als auch bei den $+\mathrm{R} /$ Pflanzen bis auf $5,1 \mathrm{ab}$.

Tab.117: Einfluss der Pb-Behandlung (mg/kg Boden) ohne (-R) oder mit Rhizobien (+R) auf den pH-Wert im Boden von Acacia saligna am Ende des Versuches

\begin{tabular}{|l|l|l|l|l|l|}
\hline $\mathrm{mg} \mathrm{Pb} / \mathrm{kg}$ Boden & 0,0 & 2,5 & 10 & 80 & \multirow{2}{*}{ GD 5\% } \\
\hline $\mathrm{yH}-\mathrm{R}$ & 5,8 & 5,3 & 5,1 & 5,2 & \\
\cline { 1 - 5 } $\mathrm{pH}+\mathrm{R}$ & 5,5 & 5,2 & 5,1 & 5,1 & \multirow{2}{*}{0,5} \\
\hline
\end{tabular}




\subsubsection{Casuarina equisetifolia}

Im allgemeinen wirkte sich die Beimpfung mit Frankia negativ auf die TS-Bildung im Spross (Tab.118, 119 u.120). Hingegen stimulierte die Frankia-Beimpfung die TS-Bildung in den Wurzeln im Vergleich zu den nicht beimpften Pflanzen (Tab. 121, 122 u.123), wenn auch die Unterschiede zwischen $-\mathrm{F}$ und $+\mathrm{F}$ nur in wenigen Behandlungen signifikant waren.

Tab.118-120: Einfluss der Zn-, Cd- und Pb-Behandlung (mg/kg Boden) ohne (-F) oder mit Frankia (+F) auf das Sprosstrockengewicht (g/Topf) von Casuarina equisetifolia

Tab.118: Zn-Behandlung

\begin{tabular}{|l|l|l|l|l|l|}
\hline mg Zn/kg Boden & 0,0 & 0,5 & 10 & 80 & GD 5\% \\
\hline $\begin{array}{l}\text { STG g/Topf } \\
-\mathrm{F}\end{array}$ & 1,92 & 3,76 & 3,27 & 2,28 & \\
$+\mathrm{F}$ & 1,46 & 2,25 & 2,51 & 1,40 & 1,13 \\
\hline
\end{tabular}

Tab.119: Cd-Behandlung

\begin{tabular}{|l|l|l|l|l|l|}
\hline mg Cd/kg Boden & 0,0 & 1,25 & 5 & 20 & GD 5\% \\
\hline $\begin{array}{l}\text { STG g/Topf } \\
-\mathrm{F}\end{array}$ & 1,92 & 3,29 & 2,11 & 1,76 & \\
$+\mathrm{F}$ & 1,46 & 2,52 & 1,37 & 1,51 & 1,38 \\
\hline
\end{tabular}

Tab.120: Pb-Behandlung

\begin{tabular}{|l|l|l|l|l|l|}
\hline mg Pb/kg Boden & 0,0 & 2,5 & 10 & 80 & GD 5\% \\
\hline $\begin{array}{l}\text { STG g/Topf } \\
-\mathrm{F}\end{array}$ & 1,92 & 3,39 & 4,41 & 4,82 & \\
$+\mathrm{F}$ & 1,46 & 3,29 & 4,04 & 4,69 & 1,37 \\
\hline
\end{tabular}

Insgesamt lagen die Sprosstrockengewichte der beimpften und unbeimpften CasuarinaPflanzen bei steigenden Schwermetall-Zugaben entweder etwas höher oder gleich hoch wie in der Kontrolle. Ebenso waren die STG der unbeimpften Pflanzen im allgemeinen etwa höher oder gleich hoch wie bei den beimpften Pflanzen. 
Tab.121-123: Einfluss der Zn-, Cd- und Pb-Behandlung (mg/kg Boden) ohne (-F) oder mit Frankia $(+\mathrm{F})$ auf das Wurzeltrockengewicht (g/Topf) von Casuarina equisetifolia

Tab.121: Zn-Behandlung

\begin{tabular}{|l|l|l|l|l|l|}
\hline mg Zn/kg Boden & 0,0 & 0,5 & 10 & 80 & GD 5\% \\
\hline $\begin{array}{l}\text { WTG g/Topf } \\
-\mathrm{F}\end{array}$ & 0,66 & 1,08 & 0,83 & 0,42 & \\
$+\mathrm{F}$ & 0,84 & 1,14 & 1,17 & 0,42 & 0,63 \\
\hline
\end{tabular}

Tab.122: Cd-Behandlung

\begin{tabular}{|l|l|l|l|l|l|}
\hline mg Cd/kg Boden & 0,0 & 1,25 & 5 & 20 & GD 5\% \\
\hline $\begin{array}{l}\text { WTG g/Topf } \\
- \text { F } \\
+\mathrm{F}\end{array}$ & 0,66 & 0,74 & 0,47 & 0,25 & \\
\hline
\end{tabular}

Tab.123: Pb-Behandlung

\begin{tabular}{|l|l|l|l|l|l|}
\hline mg Pb/kg Boden & 0,0 & 2,5 & 10 & 80 & GD 5\% \\
\hline $\begin{array}{l}\text { WTG g/Topf } \\
-\mathrm{F}\end{array}$ & 0,66 & 1,15 & 1,62 & 1,03 & \\
$+\mathrm{F}$ & 0,84 & 1,83 & 1,54 & 1,94 & 0,82 \\
\hline
\end{tabular}

Das Wurzeltrockengewicht stieg bei allen Schwermetall-Stufen gegenüber der Kontrolle an, war jedoch bei der höchsten Stufe von $\mathrm{Zn}$ oder $\mathrm{Cd}$ niedriger als bei der Kontrolle. Die Unterschiede waren allerdings nicht signifikant.

Die N-Konzentrationen im Spross der beimpften Pflanzen stieg gegenüber der Kontrolle mit zunehmenden $\mathrm{Zn}$ oder $\mathrm{Pb}-$ Gaben zunächst an, danach nahmen sie leicht ab (Tab.124 u.126). Hingegen nahm die N-Konzentration im Spross der unbeimpften Pflanzen bis zur mittleren Stufe von $\mathrm{Zn}$ oder $\mathrm{Pb}$ gegenüber der Kontrolle ab. Die höchsten Stufen von $\mathrm{Zn}$ oder $\mathrm{Pb}$ führten zu Steigerungen in den N-Konzentrationen der unbeimpften Pflanzen im Vergleich zur Kontrolle. Mit zunehmender Cd-Applikation stieg die N-Konzentration im Spross der unbeimpften Pflanzen stets bei allen Stufen gegenüber der Kontrolle und den beimpften Casuarina-Pflanzen an, wohingegen die N-Konzentration der beimpften Pflanzen mit Ausnahme der Cd-Stufe 5 ppm im Vergleich zur Kontrolle absank (Tab.125). Im allgemeinen waren kaum signifikante Unterschiede in der N-Konzentration der verschiedenen Behandlungen zu finden. Deshalb entsprach die N-Aufnahme weitgehend dem Wachstum. 
Tab.124-126: Einfluss der Zn-, Cd- und Pb-Behandlung (mg/kg Boden) ohne (-F)oder mit Frankia (+F) auf die Konzentration (mg/g TS) und Aufnahme (mg/Topf) von N im Spross von Casuarina equisetifolia

Tab.124: Zn-Behandlung

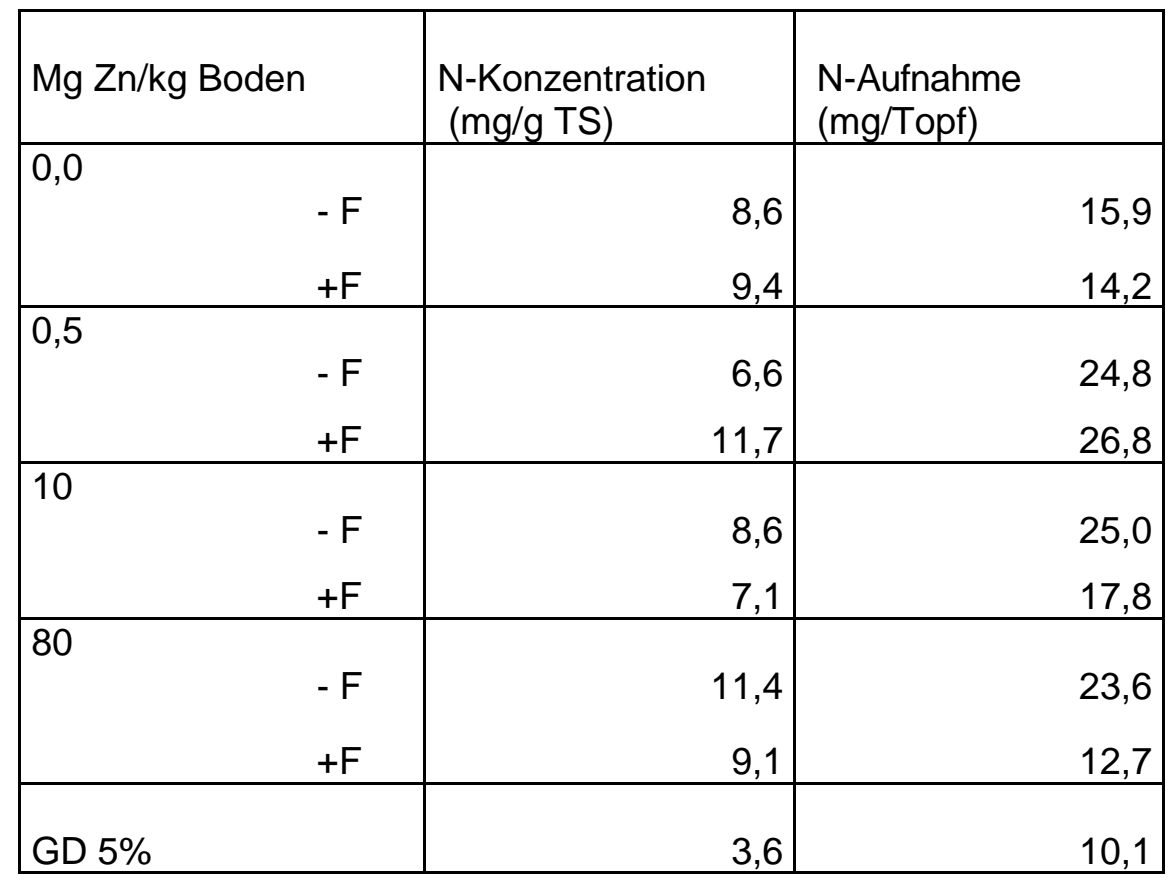

Tab.125: Cd-Behandlung

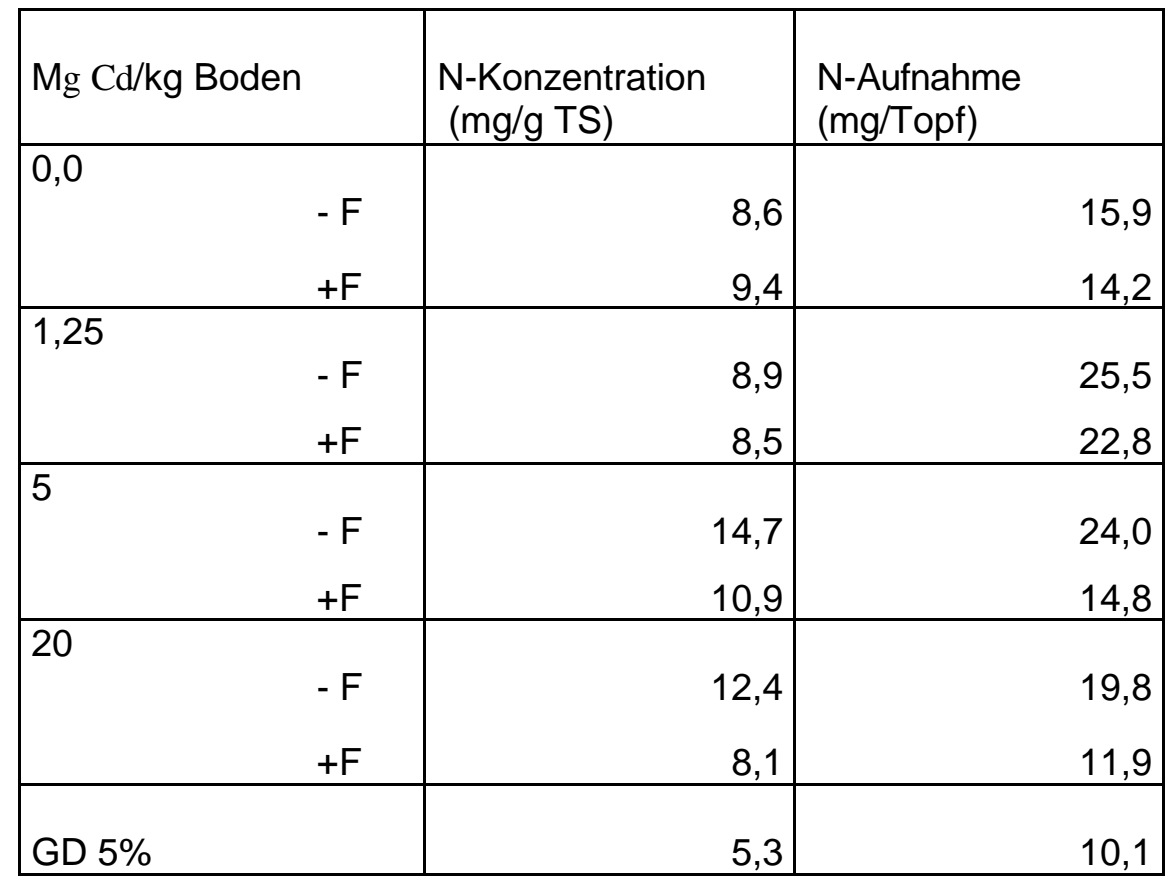


Tab.126: Pb-Behandlung

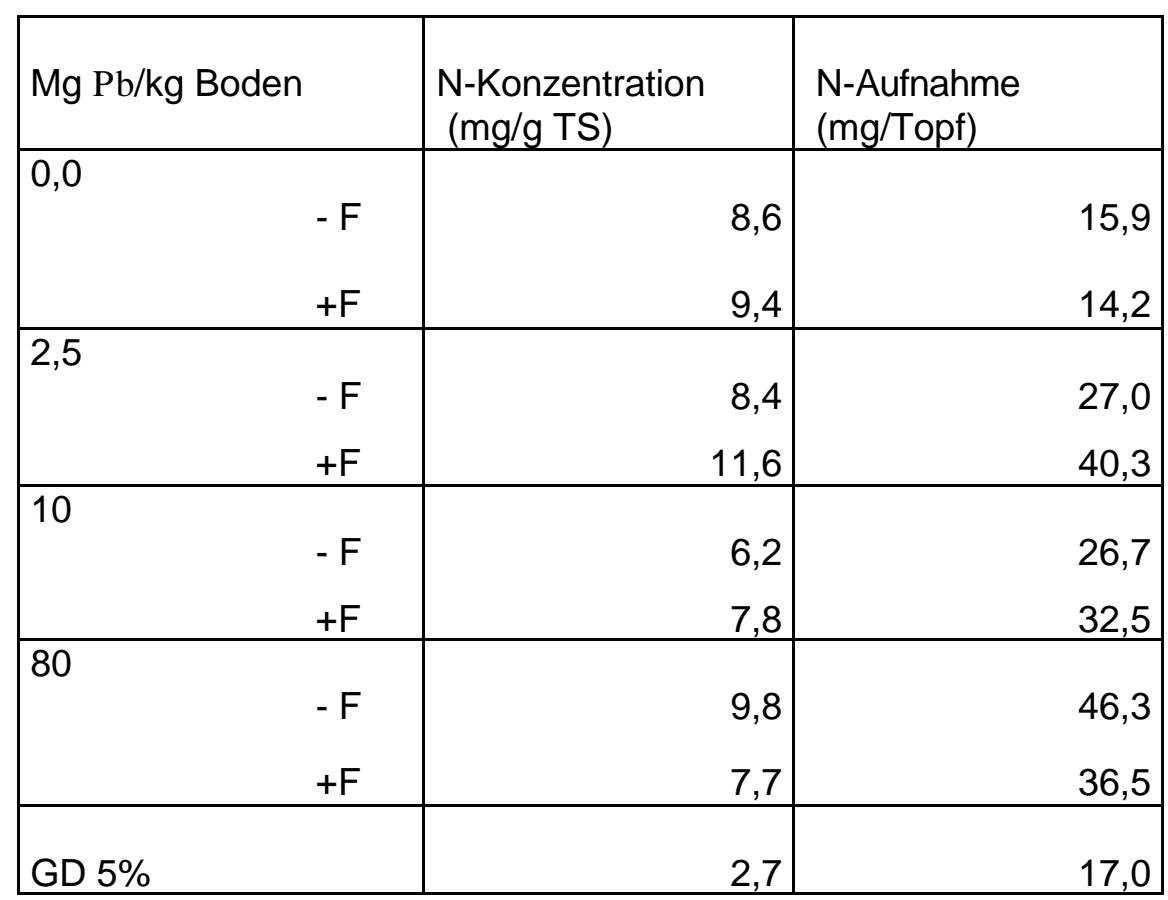

Die P-Konzentrationen zeigten beim Spross der unbeimpften und beimpften Pflanzen eine deutliche Verringerung mit zunehmender Zugabe von $\mathrm{Zn}, \mathrm{Cd}$ und $\mathrm{Pb}$ im Vergleich $\mathrm{zu}$ den Kontrollen (Tab.127, 128 u.129). Die P-Konzentrationen der beimpften Pflanzen lagen jedoch höher oder gleich hoch wie bei den unbeimpften Pflanzen.

Die K-Konzentration nahm im Spross mit steigender Zugabe an $\mathrm{Zn}, \mathrm{Cd}$ und $\mathrm{Pb}$ gegenüber der Kontrolle ab (Tab. 127, 128 u.129). Die Abnahme war allerdings nicht signifikant, weder bei den beimpften noch bei den unbeimpften Pflanzen. Nur bei $\mathrm{Pb}$ ist bei den beimpften Pflanzen eine signifikante Abnahme zu finden.

Die Ca-Konzentration nahm im Spross der beimpften und unbeimpften Pflanzen mit steigender Zufuhr der Schwermetalle $\mathrm{Zn}, \mathrm{Cd}$ und $\mathrm{Pb}$ gegenüber der Kontrolle ab. Alle Werte lagen jedoch bei den unbeimpften Pflanzen etwas höher oder gleich hoch wie bei den beimpften Pflanzen.

Die Mg-Konzentration verringerte sich meistens im Spross der beimpften und unbeimpften Pflanzen mit zunehmender Konzentration den Schwermetallen $\mathrm{Zn}, \mathrm{Cd}$ und $\mathrm{Pb}$ im Boden gegenüber der Kontrolle. 
Tab.127-129: Einfluss der Zn-, Cd- und Pb-Behandlung (mg/kg Boden) ohne (-F) oder mit Frankia $(+\mathrm{F})$ auf die Konzentration (mg/g TS) und Aufnahme (mg/Topf) von $\mathrm{P}, \mathrm{K}, \mathrm{Ca}$ und $\mathrm{Mg}$ im Spross von Casuarina equisetifolia

Tab.127: Zn-Behandlung

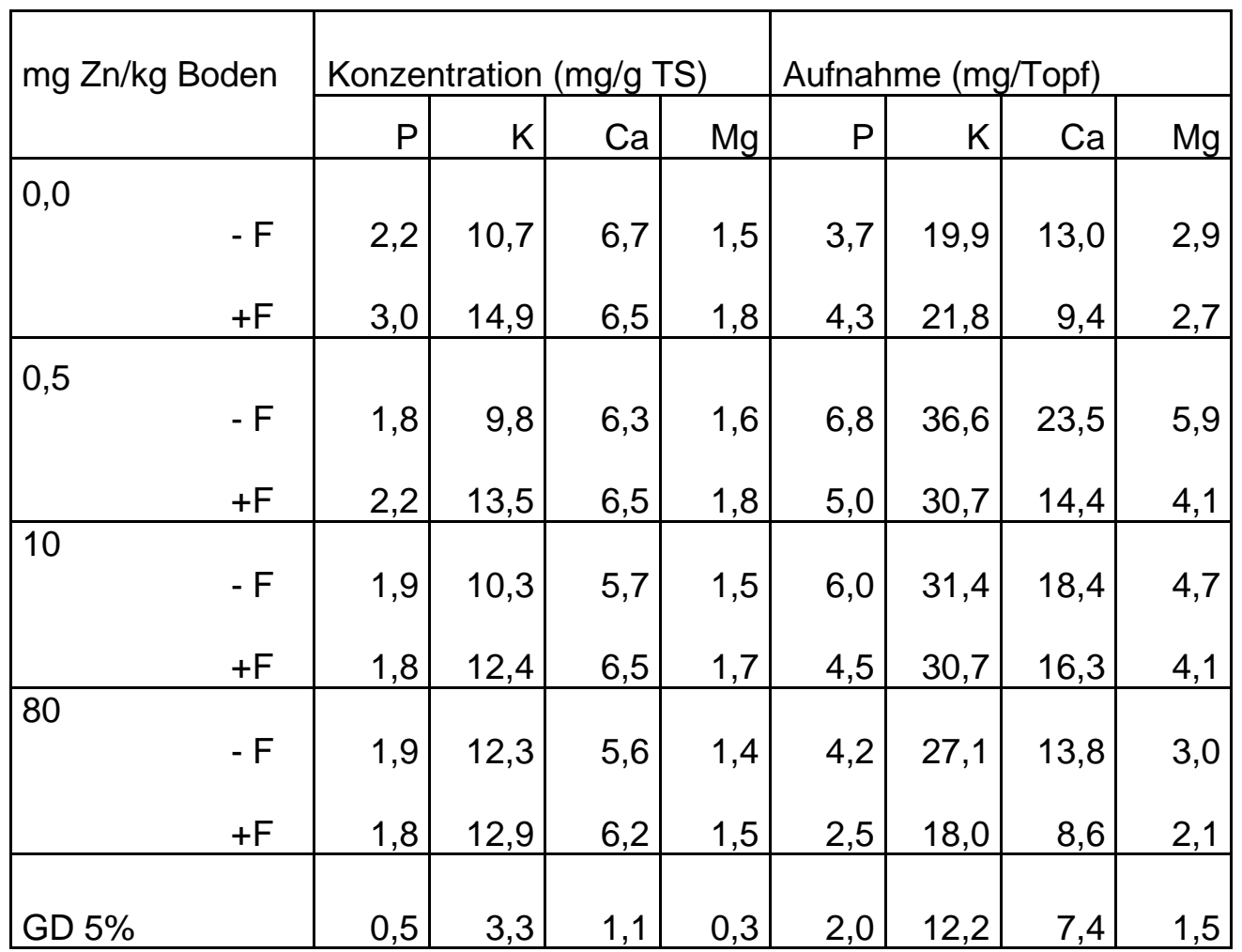

Tab.128: Cd-Behandlung

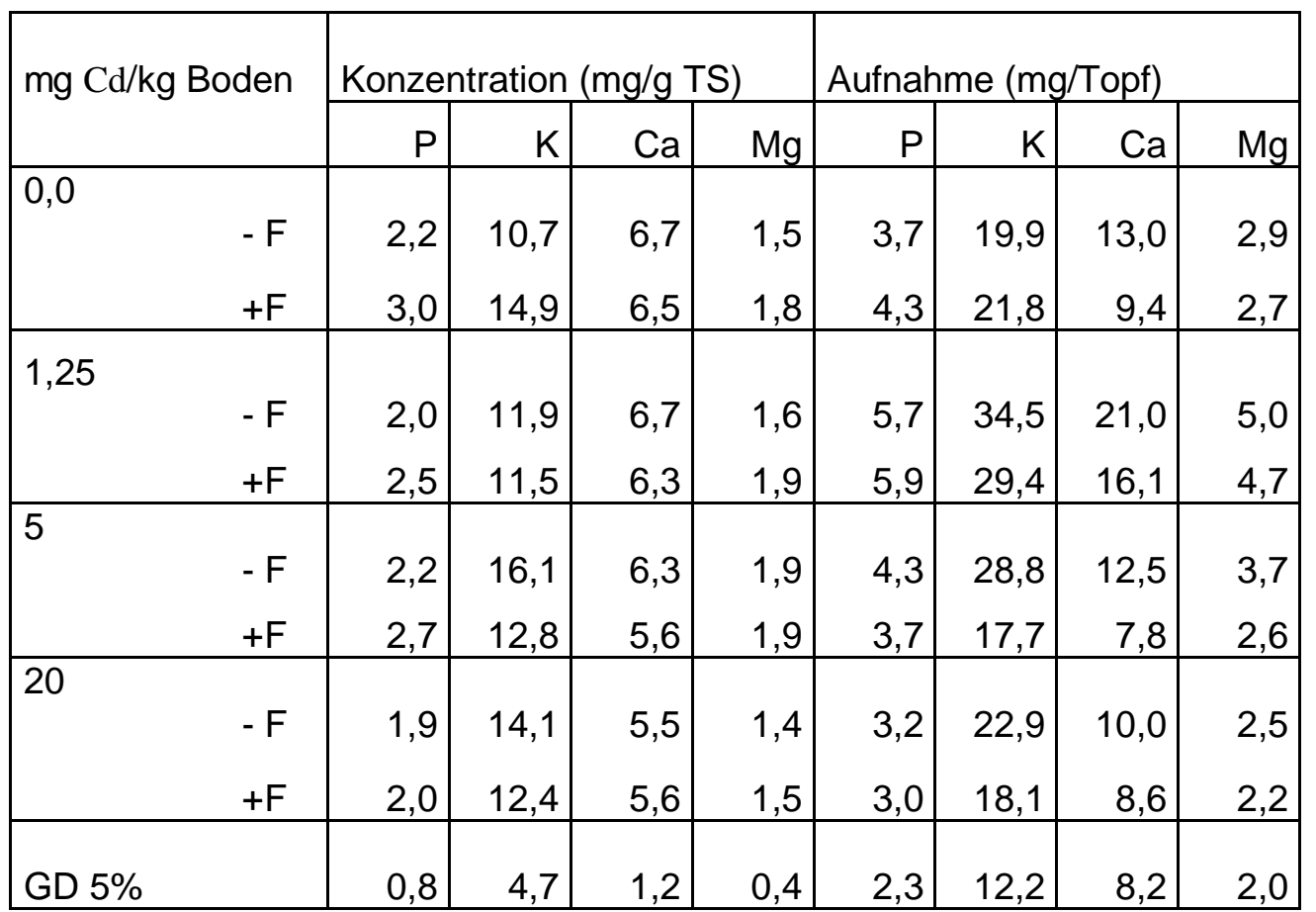


Tab.129: Pb-Behandlung

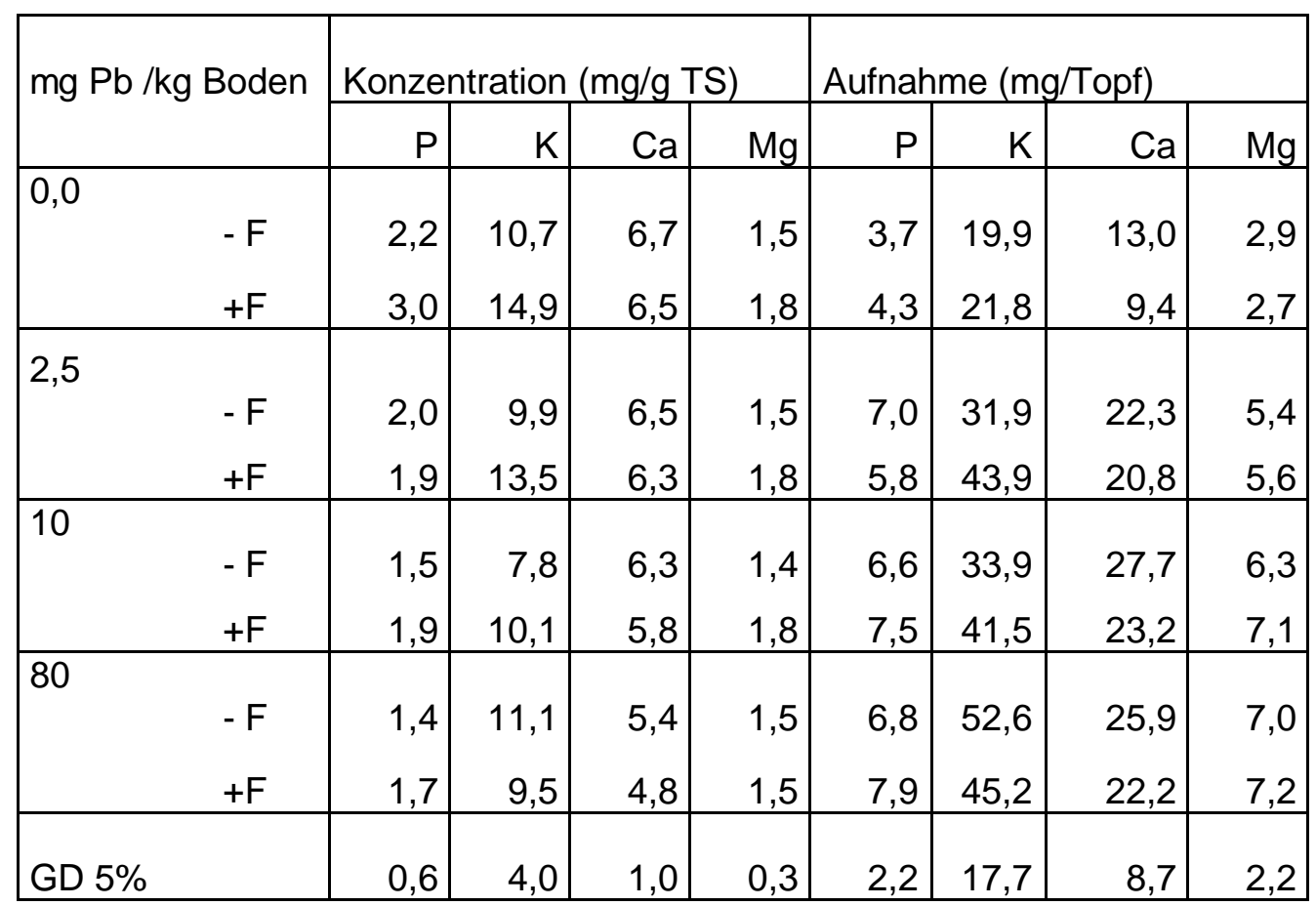

Die Aufnahmen von $\mathrm{N}, \mathrm{P}, \mathrm{K}, \mathrm{Ca}$ und $\mathrm{Mg}$ stiegen meistens im Spross der beimpften und unbeimpften Pflanzen mit steigender Schwermetall-Gabe gegenüber der Kontrolle an (127, 128 u.129). Alle Aufnahmen im Spross der beimpften Pflanzen waren höher bei der Behandlung mit $\mathrm{Pb}$ und niedriger bei der Behandlung mit $\mathrm{Zn}$ oder $\mathrm{Cd}$ als die der unbeimpften Pflanzen.

Die $\mathrm{Zn}$ oder Pb-Konzentration im Spross der beimpften und unbeimpften Pflanzen stieg mit steigender Zugabe an beiden Schwermetallen (Tab.130 u.132). Sie lag bei den beimpften Pflanzen höher als bei den unbeimpften Pflanzen. Die unbeimpften Pflanzen wiesen höhere oder ebenso hohe Zn-Aufnahme wie die beimpften Pflanzen auf. Die Pb-Aufnahme war bei den unbeimpften Pflanzen gleich oder etwas niedriger als bei den beimpften Pflanzen, was auf das Wachstum zurückzuführen ist. Die Cd-Konzentrationen und -Aufnahmen der unbeimpften Pflanzen lagen immer höher als bei den beimpften Pflanzen (Tab.131). 
Tab.130-132: Einfluss der Zn-, Cd- und Pb-Behandlung (mg/kg Boden) ohne (-F) oder mit Frankia (+F) auf die Konzentration (mg/g TS) und Aufnahme (mg/Topf) von $\mathrm{Zn}$ im Spross von Casuarina equisetifolia

Tab.130: Zn-Behandlung

\begin{tabular}{|c|c|c|c|}
\hline \multicolumn{2}{|c|}{$\mathrm{mg} \mathrm{Zn/kg} \mathrm{Boden}$} & $\begin{array}{l}\text { Zn-Konzentration } \\
\text { (mg/g TS) }\end{array}$ & $\begin{array}{l}\text { Zn-Aufnahme } \\
\text { (mg/Topf) }\end{array}$ \\
\hline \multirow[t]{2}{*}{0,0} & $-F$ & 0,09 & 0,18 \\
\hline & $+\mathrm{F}$ & 0,10 & 0,15 \\
\hline \multirow[t]{3}{*}{0,5} & & & \\
\hline & $-F$ & 0,05 & 0,20 \\
\hline & $+\mathrm{F}$ & 0,05 & 0,12 \\
\hline \multirow[t]{3}{*}{10} & & & \\
\hline & $-F$ & 0,16 & 0,53 \\
\hline & $+\mathrm{F}$ & 0,25 & 0,62 \\
\hline \multirow[t]{3}{*}{80} & & & \\
\hline & $-F$ & 0,41 & 0,83 \\
\hline & $+\mathrm{F}$ & 0,53 & 0,72 \\
\hline GD 5\% & & 0,11 & 0,25 \\
\hline
\end{tabular}

Tab.131: Cd-Behandlung

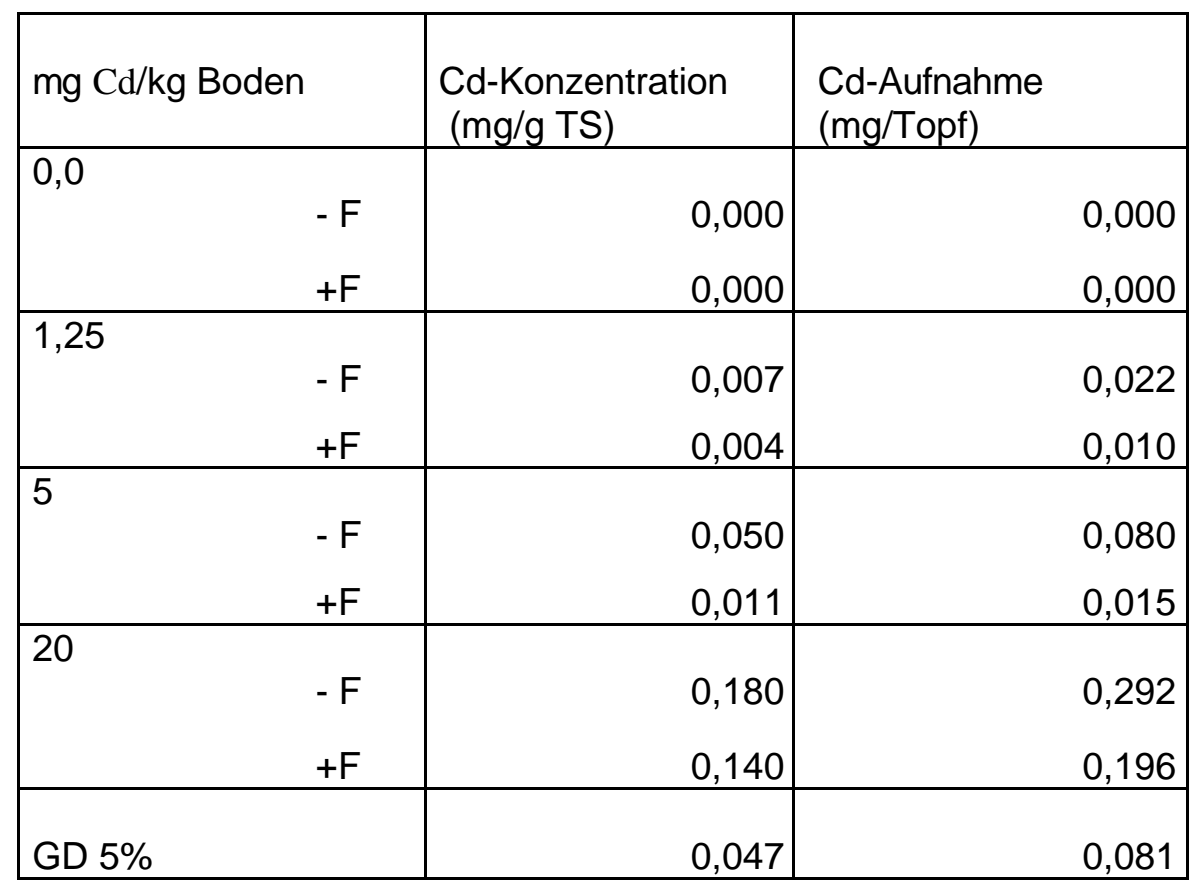


Tab.132: Pb-Behandlung

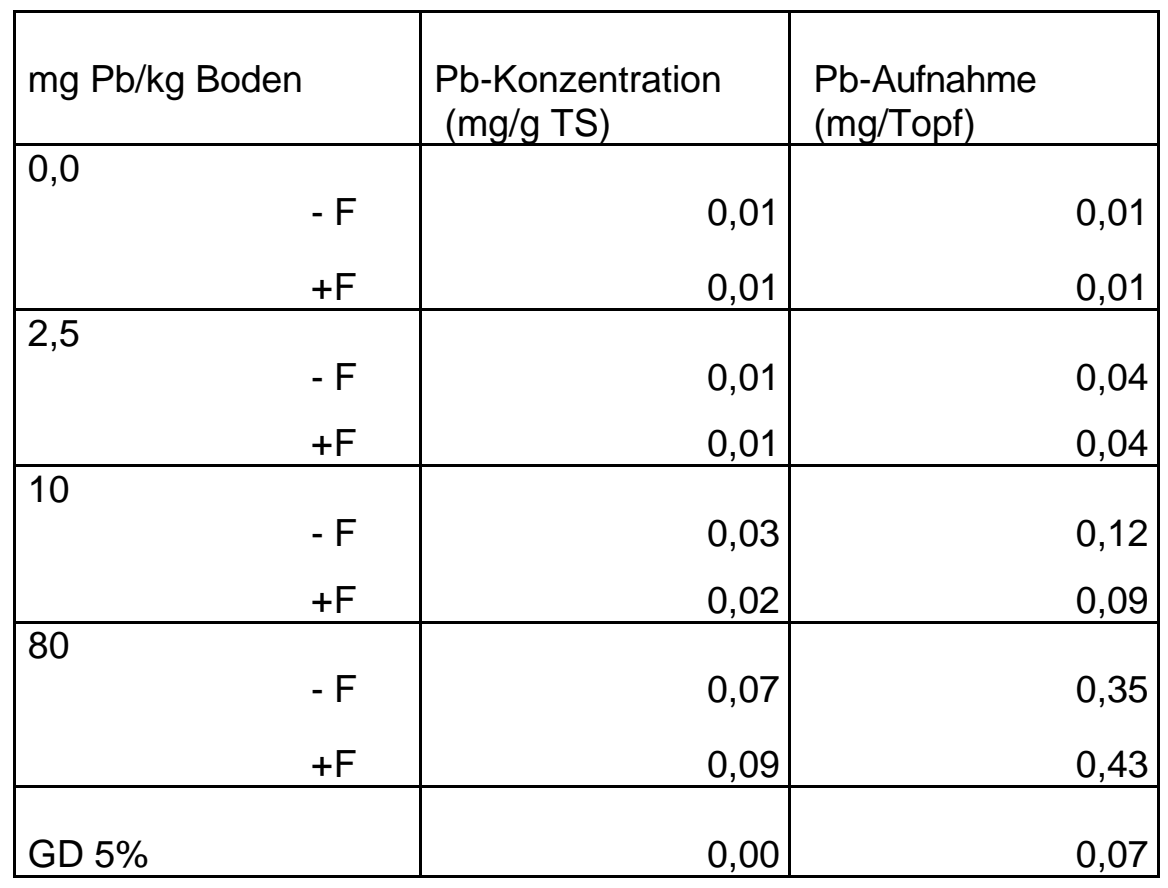

Die Konzentrationen und -Aufnahmen der Schwermetalle $\mathrm{Zn}, \mathrm{Cd}$ und $\mathrm{Pb}$ in den Wurzeln der beimpften und unbeimpften Pflanzen ließen gegenüber dem Spross ein ähnliches Bild erkennen, nur lagen die Werte der beimpften Pflanzen höher oder ebenso hoch wie bei den unbeimpften Pflanzen (Tab. 133, 134 u.135).

Tab.133-135: Einfluss der Zn-, Cd- und Pb-Behandlung (mg/kg Boden) ohne (-F) oder mit Frankia $(+\mathrm{F})$ auf die Konzentration (mg/g TS) und Aufnahme (mg/Topf) von $\mathrm{Zn}$ in der Wurzel von Casuarina equisetifolia

Tab.133: Zn-Behandlung

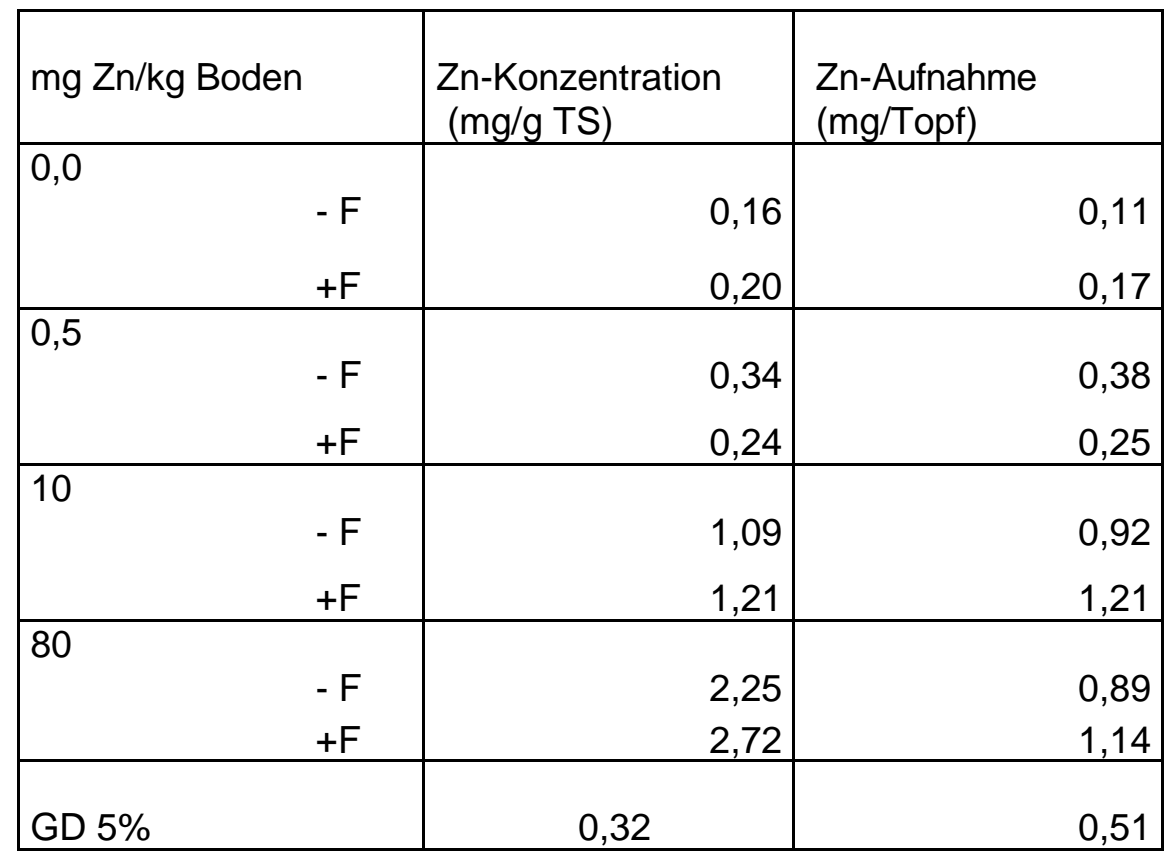


Tab.134: Cd-Behandlung

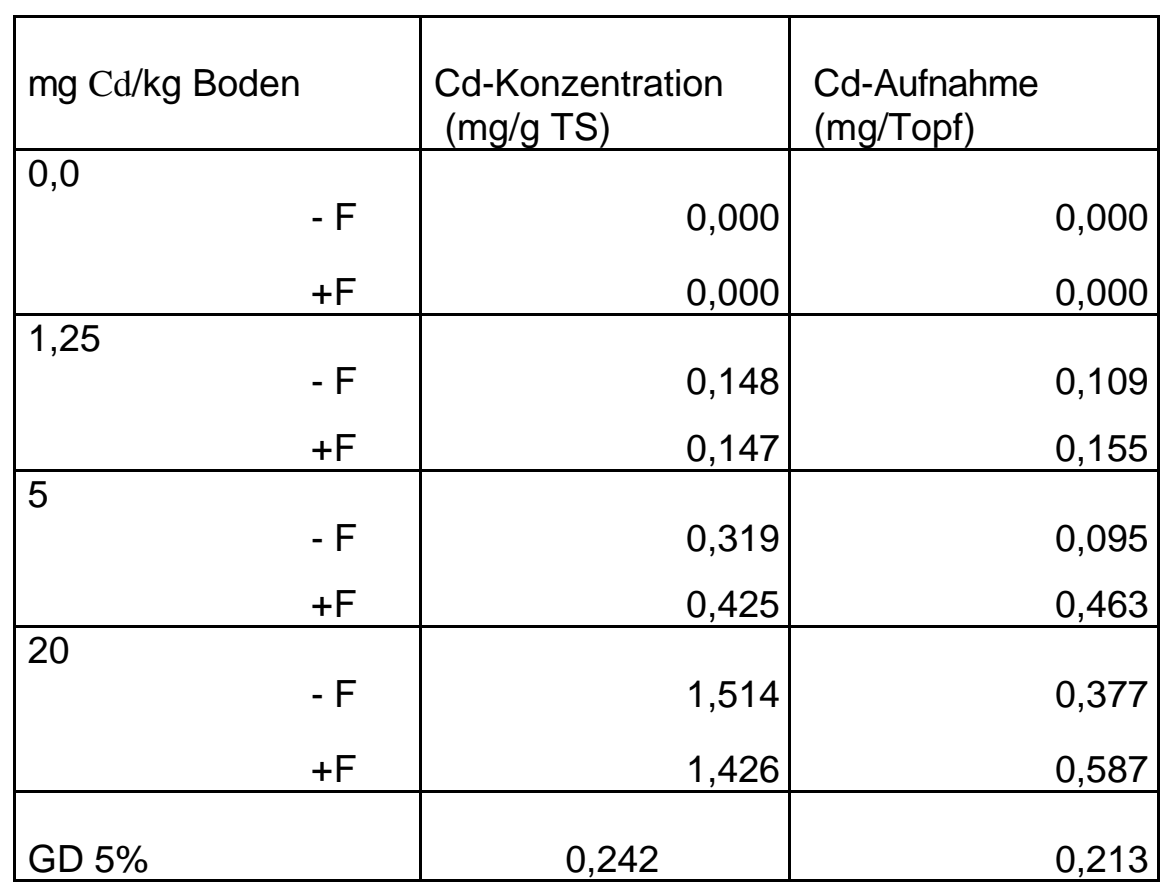

Tab.135: Pb-Behandlung

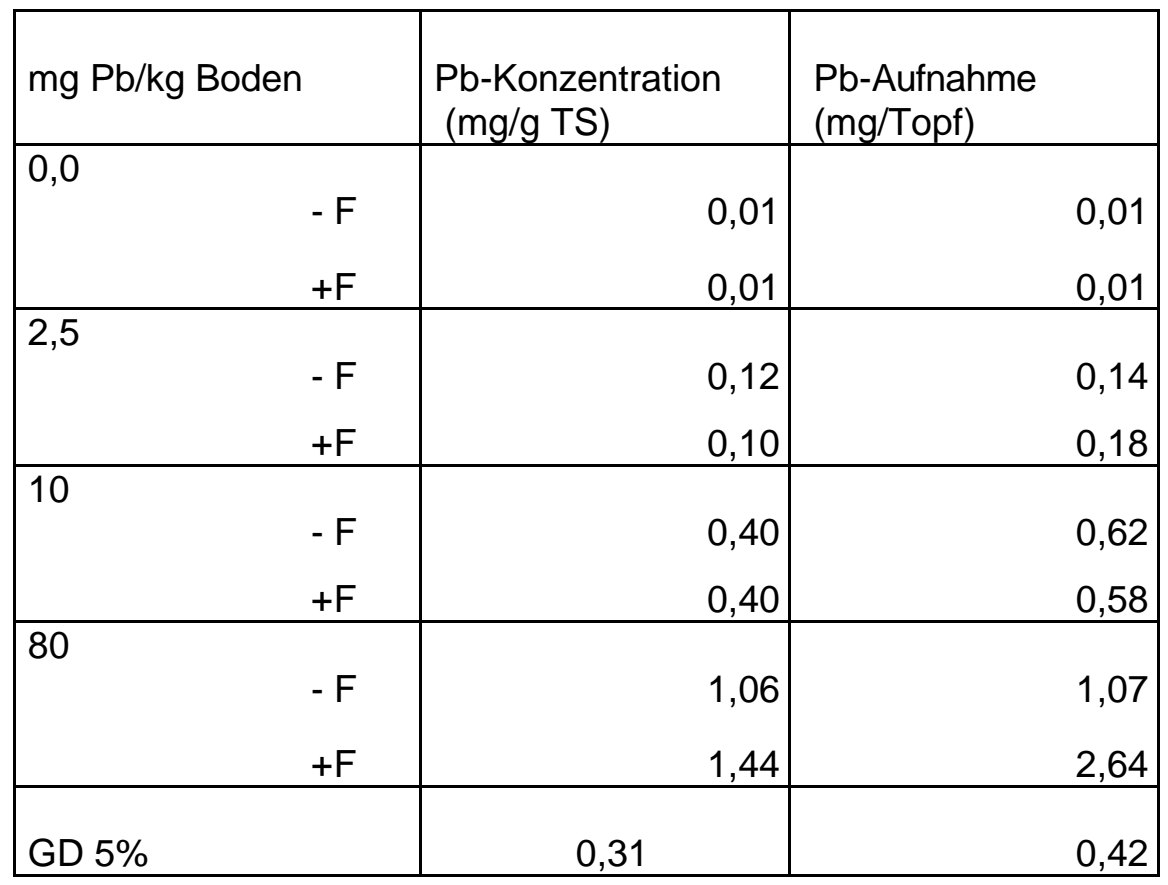

Insgesamt nahmen die $\mathrm{pH}-$ Werte des Bodens bei den beimpften und unbeimpften Pflanzen am Ende der Versuche durch steigende Schwermetall-Zugaben gegenüber der Kontrolle ab (Tab.136-138). Die Werte bei den beimpften Pflanzen waren etwas niedriger bei den ZnBehandlungen und etwas höher bei den $\mathrm{Cd}$ - oder $\mathrm{Pb}$-Behandlungen gegenüber den unbeimpften Casuarina-Pflanzen. 
Tab.136-138: Einfluss der Zn-,Cd- und Pb-Behandlung (mg/kg Boden) ohne $(-\mathrm{F})$ oder mit Frankia $(+\mathrm{F})$ auf den $\mathrm{pH}-$ Wert im Boden von Casuarina equisetifolia am Ende des Versuches

Tab.136: Zn-Behandlung

\begin{tabular}{|l|l|l|l|l|l|}
\hline $\mathrm{mg} \mathrm{Zn} / \mathrm{kg}$ Boden & 0,0 & 0,5 & 10 & 80 & \multirow{2}{*}{ GD 5\% } \\
\cline { 1 - 5 } $\mathrm{pH}-\mathrm{F}$ & 5,9 & 5,7 & 5,9 & 5,4 & \\
\cline { 1 - 5 } $\mathrm{pH}+\mathrm{F}$ & 6,0 & 5,6 & 5,9 & 5,5 & \multirow{2}{*}{0,3} \\
\hline
\end{tabular}

Tab.137: Cd-Behandlung

\begin{tabular}{|c|c|c|c|c|c|}
\hline $\mathrm{mg} \mathrm{Cd} / \mathrm{kg}$ Boden & 0,0 & 1,25 & 5 & 20 & GD 5\% \\
\hline $\mathrm{pH}-\mathrm{F}$ & 5,9 & 5,6 & 5,8 & 5,7 & \\
\hline $\mathrm{pH}+\mathrm{F}$ & 6,0 & 6,0 & 5,8 & 5,9 & 0,3 \\
\hline
\end{tabular}

Tab.138: Pb-Behandlung

\begin{tabular}{|l|l|l|l|l|l|}
\hline $\mathrm{mg} \mathrm{Pb} / \mathrm{kg}$ Boden & 0,0 & 2,5 & 10 & 80 & GD 5\% \\
\cline { 1 - 5 } $\mathrm{yH}-\mathrm{F}$ & 5,9 & 5,7 & 5,6 & 5,4 & \\
\cline { 1 - 3 }$+\mathrm{F}$ & 6,0 & 6,1 & 5,9 & 5,8 & \multirow{2}{*}{0,3}
\end{tabular}




\section{Zusammenfassender Überblick über die Ergebnisse von Versuch 3}

\section{Acacia saligna}

Durch die Beimpfung mit den $\mathrm{N}_{2}$-fixierenden Rhizobien wurde das Wachstum (Spross und Wurzel) von A. saligna bei allen Schwermetall-Behandlungen gegenüber den unbeimpften Pflanzen deutlich verbessert (Tab.97-98; 104-105 u.111-112). Das Wachstum der beimpften und unbeimpften Pflanzen wies eine deutliche Steigerung mit zunehmender Zugabe der Schwermetalle $\mathrm{Zn}, \mathrm{Cd}$ oder $\mathrm{Pb}$ bis zu der mittleren Stufe auf, danach nahm es leicht ab. Alle Werte lagen jedoch immer höher als in der Kontrolle, mit Ausnahme des Wurzel-TG der beimpften Pflanzen bei der höchsten Zn-Stufe $80 \mathrm{mg} / \mathrm{kg}$ Boden, das etwas niedriger als bei der Kontrolle war.

Aus Tab. 139 geht hervor, dass die beimpften und unbeimpften Acacia-Pflanzen ähnliche Konzentrationen des jeweiligen Schwermetalls im Boden bei der Produktion höchster STG und WTG tolerierten. Die Nährstoffaufnahme entsprach weitgehend dem Wachstum.

Tab.139: Schwermetallapplikationen die zu der höchsten Spross-, Wurzel Massen und Nährstoffaufnahmen von Acacia saligna führten (Optimale Wachstumsbedingungen)

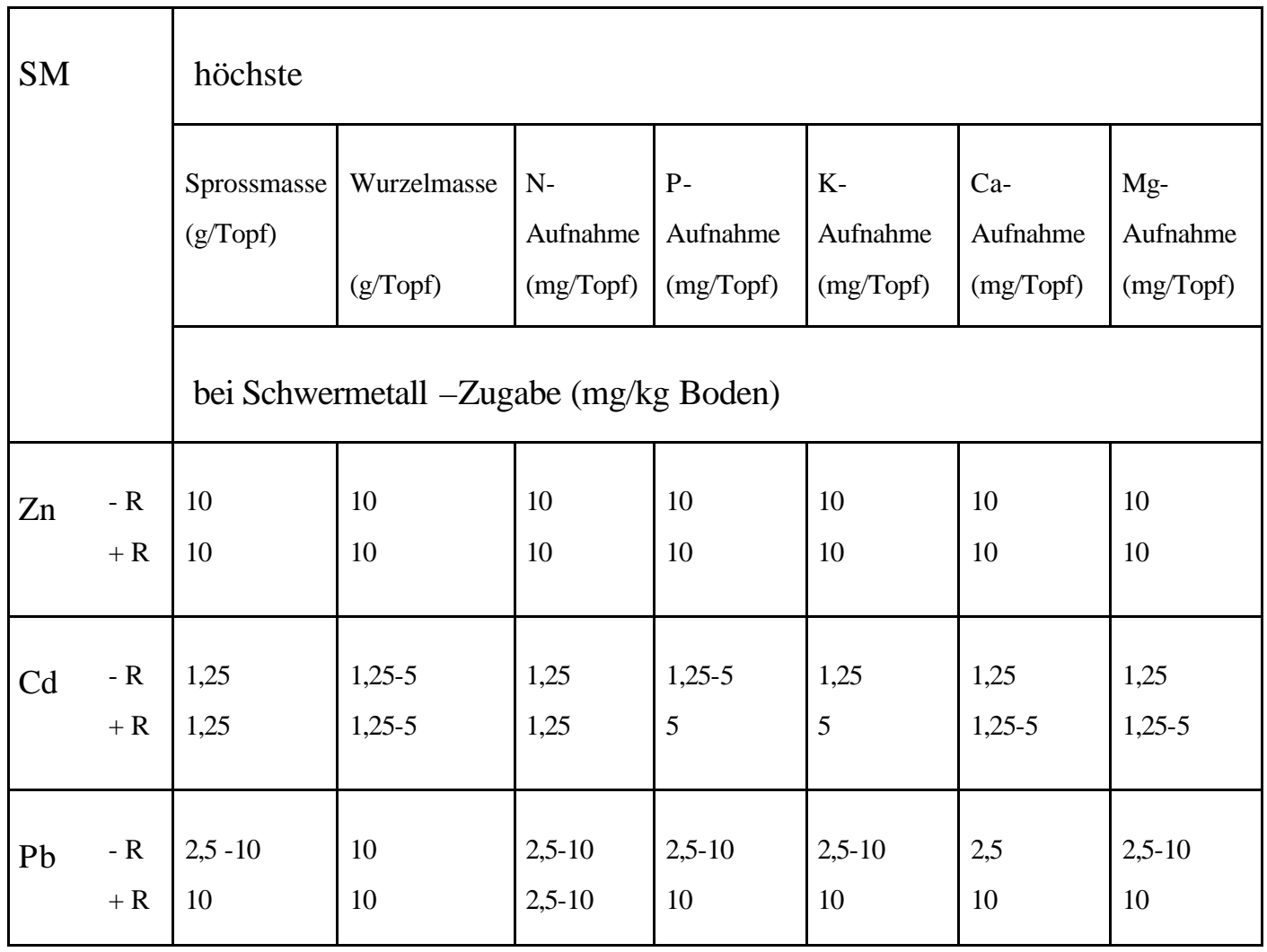

$\pm \mathrm{R}=$ mit oder ohne Rhizobien $\mathrm{SM}=$ Schwermetalle 
Tab.140: Das Verhältnis der Werte bei den höchsten bzw. toxischen Schwermetall-Gaben in \% zu den höchsten Werten (optimal =100) bei verschiedenen Schwermetall-Gaben.

\begin{tabular}{|c|c|c|c|c|c|c|c|c|}
\hline \multirow[t]{2}{*}{ SM } & \multirow[t]{2}{*}{ HSMG } & $\begin{array}{l}\text { STG } \\
(\%)\end{array}$ & $\begin{array}{c}\text { WTG } \\
(\%)\end{array}$ & $\begin{array}{l}\mathrm{N}- \\
\text { Aufnahme } \\
(\%)\end{array}$ & $\begin{array}{l}\text { P- } \\
\text { Aufnahme } \\
(\%)\end{array}$ & $\begin{array}{l}\text { K- } \\
\text { Aufnahme } \\
(\%)\end{array}$ & $\begin{array}{l}\text { Ca- } \\
\text { Aufnahme } \\
(\%)\end{array}$ & $\begin{array}{l}\mathrm{Mg}- \\
\text { Aufnahme } \\
(\%)\end{array}$ \\
\hline & & \multicolumn{7}{|c|}{ Die Werte $(\%)$ bei höchsten SMG zu den Werten bei Optimaler SMG } \\
\hline $\mathrm{Zn}$ & $80 \mathrm{mg} / \mathrm{kgBoden}$ & & & & & & & \\
\hline $\mathrm{Cd}$ & $-\mathrm{R}$ & $19 \%$ & $20 \%$ & $13 \%$ & $1 \%$ & $21 \%$ & $30 \%$ & $39 \%$ \\
\hline \multirow[t]{7}{*}{$\mathrm{Pb}$} & $+\mathrm{R}$ & $51 \%+*$ & $11 \%$ & $21 \%$ & $80 \%$ & $54 \%+*$ & $44 \%$ & $45 \%$ \\
\hline & $20 \mathrm{mg} / \mathrm{kgBoden}$ & & & & & & & \\
\hline & $-\mathrm{R}$ & $26 \%$ & $\underline{42 \%}$ & $25 \%$ & $41 \%$ & $23 \%$ & $20 \%$ & $21 \%$ \\
\hline & $+\mathrm{R}$ & $26 \%$ & $30 \%$ & $20 \%$ & $35 \%$ & $37 \%$ & $35 \%$ & $23 \%$ \\
\hline & $80 \mathrm{mg} / \mathrm{kgBoden}$ & & & & & & & \\
\hline & $-\mathrm{R}$ & $49 \%$ & $38 \%$ & $46 \%$ & $57 \%$ & $51 \%$ & $58 \%$ & $56 \%$ \\
\hline & $+\mathrm{R}$ & $64 \%+*$ & $45 \%+*$ & $49 \%+*$ & $35 \%$ & $56 \%$ & $54 \%$ & $57 \%$ \\
\hline
\end{tabular}

$\mathrm{SM}=$ Schwermetalle SMG $=$ Schwermetall - Gaben HSMG $=$ Höchste Schwermetall Gaben

$-*=$ signifikant niedriger als Kontrolle, also " toxischer " Effekt $+_{*}=$ signifikant höher als Kontrolle

Tab.140 stellt die Prozentwerte bei den höchsten bzw. toxischen Schwermetall-Gaben zu den höchsten Werten (optimal =100) bei verschiedenen Schwermetall-Gaben dar.

Die beimpften Acacia-Pflanzen zeigten allgemein bei allen Schwermetall-Behandlungen gegenüber den unbeimpften Pflanzen in den Sprossen wegen des verbesserten Wachstums höhere Aufnahmen der Elemente N, P, K, Ca, Mg und der Schwermetalle (mit Ausnahme des $\mathrm{Pb}$ ), wohingegen die Konzentrationen dieser Elemente bei den beimpften Pflanzen niedriger als bei den unbeimpften Pflanzen (Verdünnungseffekt) waren.

Bei allen Schwermetall-Stufen lag die N-Konzentration in den Sprossen der unbeimpften Pflanzen immer höher oder zumindest gleich hoch wie bei der Kontrolle, wohingegen die NKonzentration im Spross der beimpften Pflanzen immer niedriger oder gleich hoch wie in der Kontrolle war. Ein direkter Einfluss der $\mathrm{N}_{2}$-Fixierung zeigte sich nach der Inokulation mit Rhizobien durch das verbesserte Wachstum der +R/Pflanzen.

Insgesamt nahm die Konzentration von $\mathrm{P}, \mathrm{K}, \mathrm{Ca}$ und $\mathrm{Mg}$ in den Sprossen der beimpften und unbeimpften Acacia-Pflanzen durch steigende Schwermetall-Gaben zunächst ab, dann bei der 
höchsten Stufe des Schwermetalls wieder zu. Alle Werte lagen jedoch niedriger als bei der Kontrolle.

Im allgemeinen nahmen die Aufnahmen von $\mathrm{N}, \mathrm{P}, \mathrm{K}, \mathrm{Ca}, \mathrm{Mg}$ und den Schwermetallen $\mathrm{Zn}, \mathrm{Cd}$ und $\mathrm{Pb}$ in den Sprossen der beimpften und unbeimpften Pflanzen mit steigenden Schwermetallgaben bis zur mittleren Stufe gegenüber der Kontrolle zu. Die Aufnahmen dieser Elemente lagen bei der höchsten Stufe der Schwermetalle entweder höher oder gleich hoch wie bei der Kontrolle.

Eine Steigerung der Schwermetall-Konzentration im Boden führte $\mathrm{zu}$ sehr starken Erhöhungen ihrer Konzentration und Aufnahme in den Sprossen und Wurzeln der beimpften und unbeimpften Pflanzen gegenüber der Kontrolle.

Wie in den Versuchen 1 und 2, zeigte sich in allen Varianten, dass die pH-Werte des Bodens bei den Schwermetall-Behandlungen am Ende des Versuches abnahmen, obwohl sie hier etwas höher als in 1. und 2. Versuch lagen. Die pH-Werte des Bodens der unbeimpften Pflanzen waren etwas höher als im Boden der beimpften Pflanzen. Alle pH-Werte lagen jedoch niedriger als bei der jeweiligen Kontrolle (Tab. 103, 110 u.117).

\section{Casuarina equisetifolia}

Die Beimpfung mit Frankia zeigte bei allen $\mathrm{Zn}, \mathrm{Cd}$ und $\mathrm{Pb}$-Behandlungen einen deutlich negativen Einfluss auf das Wachstum, vermutlich wegen des niedrigeren $\mathrm{pH}$-Wertes des Bodens im Verlauf des Versuches (Tab.136, 137 u.138), obwohl das Wurzeltrockengewicht der beimpften Casuarina-Pflanzen bei allen Schwermetall-Behandlungen aufgrund der Knöllchenbildung durch Frankia gegenüber den unbeimpften Pflanzen höher lag. Die gebildeten Knöllchen konnten demnach das Wachstum der beimpften Pflanzen nicht fördern.

Da die Spross-TG der unbeimpften Pflanzen bei allen Schwermetall-Behandlungen fast immer höher oder zumindest gleich hoch lagen wie bei den beimpften Pflanzen (Tab.118, 119 u.120), ergaben sich daraus erhöhte Aufnahmen der Schwermetalle Zn und Cd im Spross dieser Pflanzen (Tab.130 u 131). Hingegen wurde eine ebenso hohe Pb-Aufnahme im Spross der -F/Pflanzen, mit Ausnahme der Pb-Stufe 80 ppm, wie in den +F/Pflanzen festgestellt (Tab. 132).

Insgesamt nahm das Wachstum der beimpften und unbeimpften Casuarina-Pflanzen durch steigende Schwermetall-Konzentration im Boden zunächst zu und dann bei höchsten Stufen wieder ab. Alle Werte lagen jedoch immer höher als in der Kontrolle.

Sowohl bei den Acacia- als auch bei den Casuarina-Pflanzen nahmen die Boden-pH-Werte bis zum Ende des Versuches ab. Es wird vermutet, dass diese Absenkung des Boden-pH die 
negative Wirkung der Beimpfung der Casuarina-Pflanzen mit Frankia verursachte, da Frankia auf Bodenazidität empfindlich reagiert (s. Diskussion). 


\section{Diskussion}

Ein wichtiges Problem des Landbaus in Ägypten ist die Umweltverschmutzung, die durch wachsende Industrialisierung vieler Regionen des Landes verursacht wurde und noch wird. Dadurch sind große Anbauflächen nicht mehr kultivierbar. Ein Hauptfaktor der Umweltverschmutzung ist die Anreicherung des Bewässerungswassers mit Schwermetallen, die durch Industrieabfälle entstanden ist. Darüber hinaus werden große Landflächen in verschiedenen Regionen Ägyptens für die Entsorgung von Industrieabfällen ohne genügende Überwachung benutzt.

Deshalb werden z.Z. verschiedene Maßnahmen zur Überwindung dieses Problems geprüft. Eine dieser Maßnahmen ist die Kultivierung von Bäumen (keine Nahrungskulturen) in diesen Gebieten. Dies hat eine Doppelstrategie: einerseits werden die mit Schwermetallen kontaminierten Flächen wieder begrünt, andererseits wird ein ökonomischer Beitrag dazu geleistet, den Anbau von holzlieferenden Bäumen zu forcieren, die geringe Ansprüche an Wasser und Boden haben.

Ziel der vorliegenden Arbeit war, die Toleranz der drei wichtigsten holzlieferenden Bäume Ägyptens Acacia saligna, Casuarina equisetifolia und Cupressus sempervirens gegen hohe Konzentrationen der im Rahmen des Umweltschutzes wichtigsten Schwermetalle $\mathrm{Zn}, \mathrm{Cd}$ und $\mathrm{Pb}$ zu untersuchen, um Anhaltspunkte für ihre Eignung für den Anbau auf mit Schwermetallen kontaminierten Böden zu gewinnen.

\section{Einfluss der Schwermetalle auf das Wachstum holzliefernder Bäume}

In der Literatur sind zahlreiche Untersuchungen über der Schwermetalle zu finden, die nicht nur als Hochleistungs-Mikronährstoffe (wie z.B. Zn), sondern auch solche, die für die Pflanzen als giftige Elemente bekannt sind. Gezielte Untersuchungen, welche sich mit der Toleranz der Pflanzen gegen hohe Schwermetall-Konzentrationen im Boden und mit ihrem möglichen Anbau unter solchen Stressbedingungen, oder welche sich in diesem Zusammenhang mit holzliefernden Baumarten verschiedener Familien beschäftigen, fehlen jedoch.

\section{Einfluss des Zn}

Im ersten Versuch lieferten die Casuarina-Pflanzen das höchste STG und WTG bei ZnZugabe von 10-20 mg/kg Boden, und wiesen somit eine höhere Toleranz gegenüber steigenden Zn-Konzentrationen im Boden als die Acacia- und Cupressus-Pflanzen auf

(Tab.72). Die beiden letztgenannten Pflanzen bildeten die höchste TS in den Sprossen und Wurzeln bei Zn-Zugabe von $10 \mathrm{mg} / \mathrm{kg}$ Boden. Das heißt, die optimale Zn-Konzentration für das Wachstum lag bei Casuarina-Pflanzen bei 10 bis 20 ppm und bei Acacia- und CupressusPflanzen bei $10 \mathrm{mg} \mathrm{Zn/kg} \mathrm{Boden} \mathrm{(Abb.1).} \mathrm{Im} \mathrm{Gegensatz} \mathrm{zu} \mathrm{den} \mathrm{Casuarina-} \mathrm{und} \mathrm{Cupressus-}$ 
Pflanzen wuchsen die Acacia-Pflanzen bei der höchsten Zn-Gabe (40 mg/kg Boden in Versuch 1 und $80 \mathrm{mg} / \mathrm{kg}$ Boden in Versuch 3) wesentlich besser als die Kontroll-Pflanzen (Tab. 8-9 u.97-98).

$\mathrm{Zu}$ ähnlichen Ergebnissen sind Arora und Singh (1970) bei Psidium guava, Shaban und Eid (1982) bei Mais und Baumwolle, El-Saied et al. (1981) bei Tomaten, El-Ghamriny (1976) und El-Beheidi (1972) bei Gurken und Taha (1994) bei Parkinsonia aculeata, gekommen

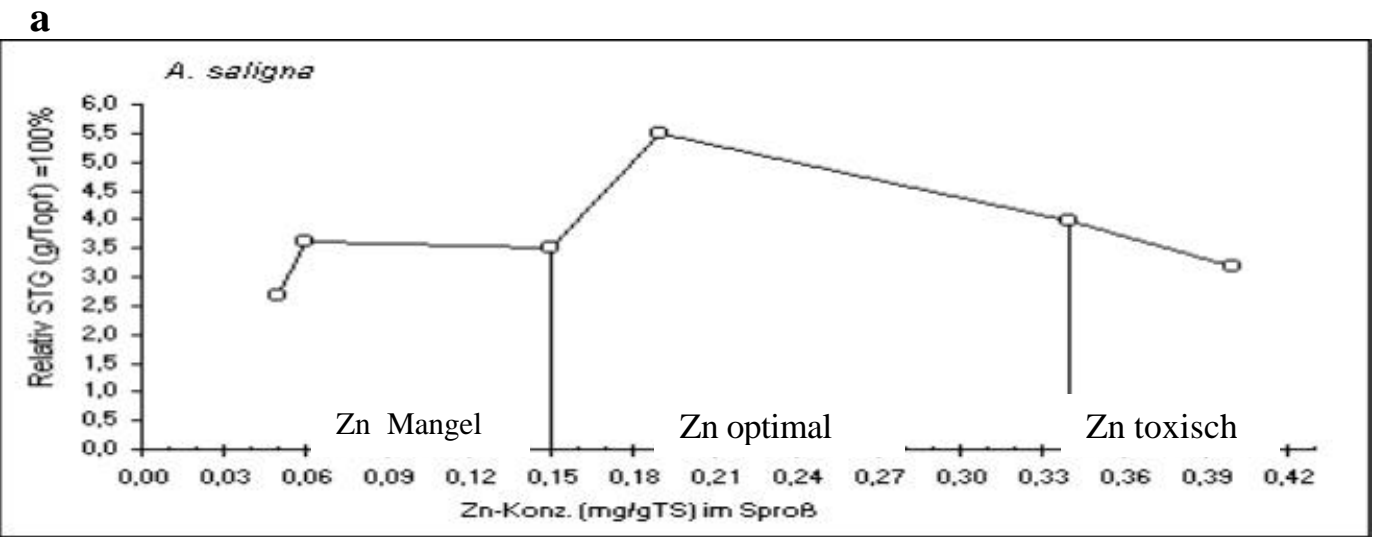

b

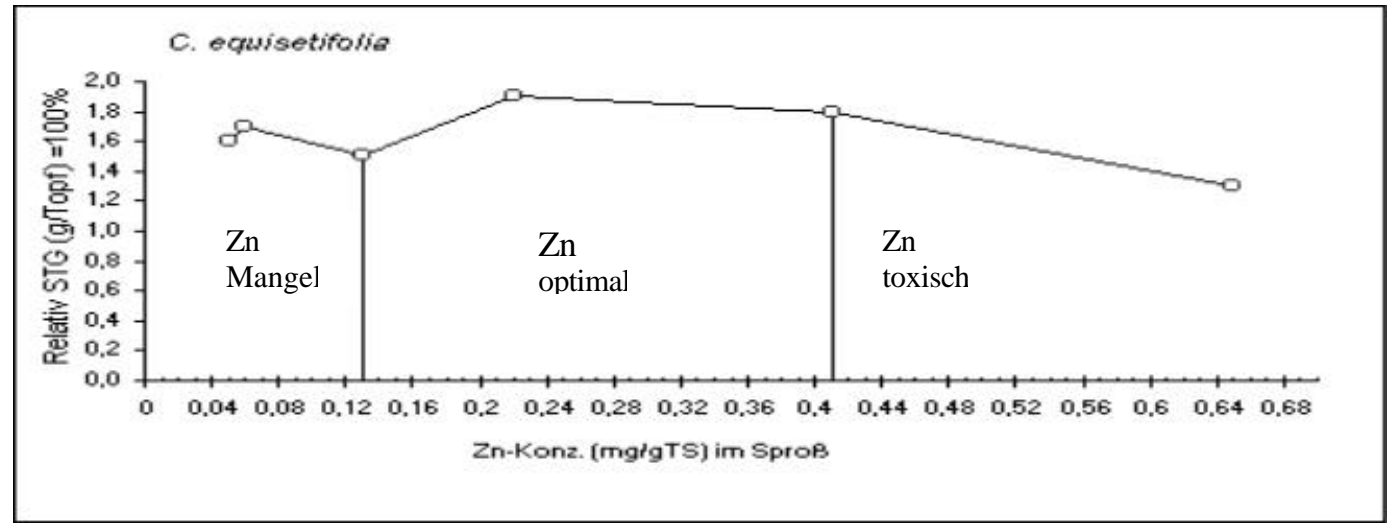

c

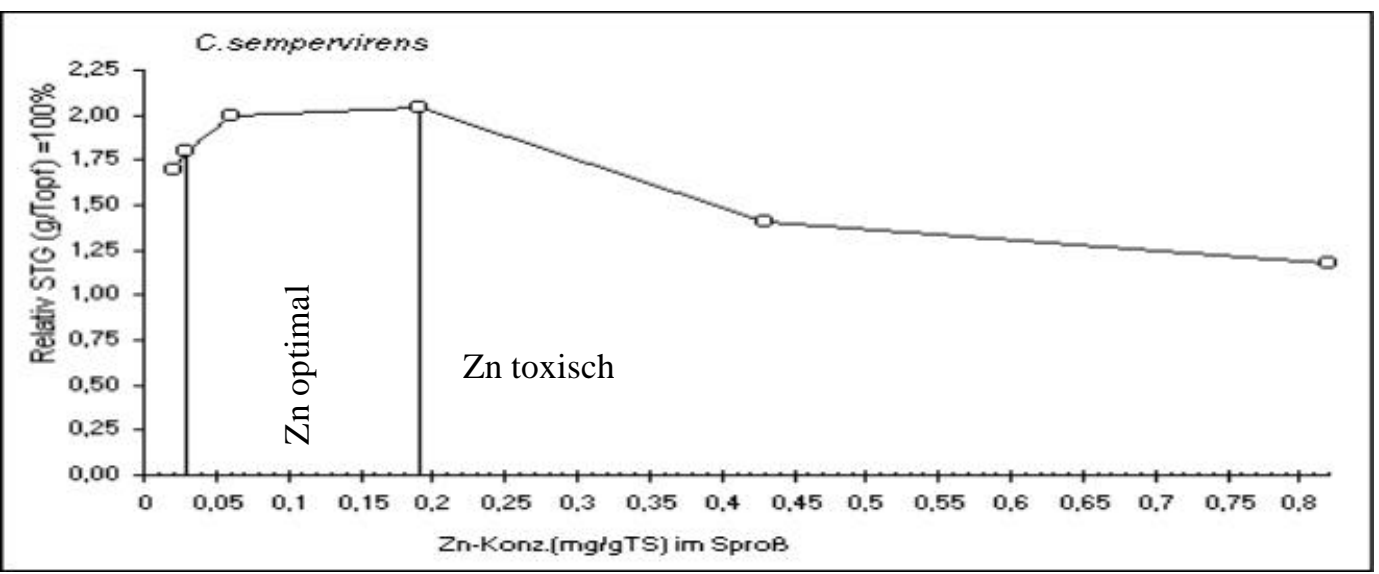

Abb.1a-c: Ableitung von Grenzwerten für die Bereiche Mangel-, optimale und Überversorgung (Toxizität) der Pflanzen mit Zn 
Die in den vorliegenden Untersuchungen festgestellte beste Wirkung der mittleren Zn-Zugabe ist für die Photosynthese der Blätter notwendig. Hu und Sparks (1990 und 1991) berichteten, dass die höchste Photosynthese im Blatt bei Zn-Gabe zum Boden von 14 ppm zu finden war. Ähnliches fanden Poroknevich (1975) und El-Hadidy et al. (1980) bei Weizen.

Durch die Beimpfung mit Rhizobien (Versuch 3) wuchsen der Acacia-Pflanzen bei allen ZnStufen wesentlich besser als die unbeimpften (Tab. 97 u. 98). Die Beimpfung mit Frankia hatte zwar einen negativen Einfluss auf das Wachstum der Casuarina-Pflanzen gegenüber den unbeimpften Pflanzen, aber das Sprosstrockengewicht der beimpften und unbeimpften Pflanzen nahm durch steigende Zn-Zufuhr gegenüber der Kontrolle zu (Tab.118). Hingegen lagen die Wurzel-TG der beimpften Pflanzen höher als die der unbeimften Pflanzen (Tab.121). Dies könnte auf die Knöllchenbildung an den Wurzeln der beimpften Pflanzen zurückgeführt werden. Vergleichbare Ergebnisse in der Literatur wurden nicht gefunden.

\section{Einfluss des Cd}

Von größter Bedeutung war die in der vorliegenden Arbeit erstmalige Feststellung, dass niedrige Cd-Zugaben von 2,5 bis $5 \mathrm{mg} / \mathrm{kg}$ Boden das Wachstum von Acacia-Pflanzen wesentlich förderten (Tab.15 u.16). Bei den Cupressus- bzw. Casuarina-Pflanzen wurde das Wachstum, wenn auch nicht signifikant zur Kontrolle, bei Cd-Zugabe von 2,5 bzw. $10 \mathrm{mg} / \mathrm{kg}$ Boden ebenfalls verbessert (Tab. 36-37 u. 57-58). Zu ähnlichen Ergebnissen kamen Costa und Spitz (1997). Sie fanden, dass die Trockengewichte von Lupinus albus nach CdBehandlung keine signifikanten Unterschiede zur Kontrolle aufwiesen. Das heißt, dass Cadmium-tolerante Pflanzen an hochbelasteten Standorten überleben können. Durch verschiedene metabolische Prozesse, wie zum Beispiel selektive Ionenaufnahme, verringerte Permeabilität der Membranen, Komplexierung und Speicherung von Metallen in verschiedenen Organen (Kompartimentierung), Änderungen im Stoffwechsel, Einbau in Polypeptide, wird ein Überschuss an Spurenelementen ertragen (Verklen und Schat, 1990; Ross und Kaye, 1994). Auch Costa und Spitz (1997) berichteten, dass niedrige CdKonzentrationen zu einer Steigerung der löslichen Kohlenhydrate (Stärke, Mannose und Raffinose) und freier Aminosäuren (Glutamat, Cystein, Glycin, Hydroxylsin, Prolin und Asparagin) im Spross führten. Van Balen et al. (1980) Franceschi und Schneren (1986) Van Steveninck und Fernando (1995) und Mazen und El Maghraby (1997 und 1998) berichteten, dass die Ca-Oxalate im Pflanzengewebe mit steigender Cd-Konzentration anstiegen, was die Pflanzentoleranz gegen Cd-Toxizität erhöhte.

Falls sich diese Feststellung unter Feldbedingungen bestätigen ließe, würde ein wesentliches Umdenken hinsichtlich des als toxisches Element bekannten $\mathrm{Cd}$ im Umweltschutz nötig sein. Auf der anderen Seite zeigten die Ergebnisse allgemein, dass weiter steigende Cd-Zugaben 
von über $5 \mathrm{mg} / \mathrm{kg}$ bis $20 \mathrm{mg} / \mathrm{kg}$ Boden in allen Versuchen und bei den drei Baumarten zu Beeinträchtigungen der TS-Bildung im Spross und in der Wurzel führten. Schon 1978 berichteten Malone et al., dass zunehmende Cd-Gaben eine Schädigung des Wurzelsystems verursachten. Barcelo et al. (1985) stellten eine Verzögerung des Wachstums durch CdApplikation fest. Die Behandlung des Bodens mit Cd hemmte das Wachstum von Tomaten (Moral et al., 1994) und Pinus sp. und Fraxinus angustifolia (Arduini et al., 1998). Yang et al. (1996) fanden, dass durch steigende Cd-Gaben über $14 \mu \mathrm{M}$ hinaus eine Verringerung des STG um $50 \%$ und des WTG um $80 \%$ gegenüber der Kontrolle verursacht wurde.

In den vorliegenden Untersuchungen zeigten die Pflanzen der drei Baumarten, besonders bei C. sempervirens, chlorotische Verfärbungen durch starke Cd-Zugaben. Ähnliche Symptome erhielten auch Arduini et al. (1998) an Pinus sp und Fraxinus angustifolia und Klobus und Buczek (1985) an Cucumus sativus. Die letztgenannten Autoren führten diese Symptome auf einen Einfluss des Cd auf den Mg- bzw. Chlorophyll-Gehalt der Blätter zurïck.

Im dritten Versuch wurde die schädigende Wirkung des $\mathrm{Cd}$ auf das Wachstum der AcaciaPflanzen durch die Beimpfung mit Rhizobien gemildert verglichen mit der Cd-Wirkung bei den unbeimpften Pflanzen (Tab. 104 u. 105). In anderen Worten, die beimpften AcaciaPflanzen konnten höhere Cd-Konzentrationen im Boden besser vertragen bzw. tolerieren. Anders war es bei den mit Frankia beimpften Casuarina-Pflanzen. Hier reagierten die beimpften Pflanzen empfindlicher auf höhere Cd-Konzentrationen im Boden als die unbeimpften (Tab. 119 u. 122). Dies könnte auf die Beschädigung der Wurzeln bzw. der Knöllchen an den Wurzeln der beimpften Pflanzen durch das Cd zurückgeführt werden. Die Knöllchen verfaulten und wurden schwarz. Für diese Befunde sind kaum vergleichbare Angaben in der Literatur zu finden.

Einfluss des $\mathrm{Pb}$

Mit Ausnahme der Acacia-Pflanzen, bei denen das Wachstum bei Pb-Zugabe von $40 \mathrm{mg} / \mathrm{kg}$ Boden, wenn auch nicht bedeutend, im Vergleich zur Kontrolle herabgesetzt wurde, wirkte sich die steigende $\mathrm{Pb}$-Zugabe bis zur genannten Stufe fördernd auf das Wachstum der Pflanzen der drei Baumarten aus (Tab.22-23, 43-44 u. 64-65). Ebenfalls im 2. Versuch, in dem die $\mathrm{N}$-Menge des verwendeten $\mathrm{Pb}\left(\mathrm{NO}_{3}\right)_{2}$ bei der $\mathrm{N}$-Düngung der Pflanzen berücksichtigt wurde, führten die getesteten $\mathrm{Pb}$-Behandlungen bis $40 \mathrm{mg} / \mathrm{kg}$ Boden zu einer Förderung des Wachstums der Pflanzen der drei Baumarten. Der enthaltende Stickstoff im Bleinitrat beeinflusste kaum die Reaktion der Pflanzen der drei Baumarten auf steigende $\mathrm{Pb}$ - 
Konzentration im Boden. Die Acacia-Pflanzen reagierten im dritten Versuch empfindlich auf die Zufuhr von $\mathrm{Pb}$ zum Boden in Höhe von $80 \mathrm{mg} / \mathrm{kg}$ Boden. Diese Behandlung beeinträchtigte ebenfalls das Wachstum sowohl bei den mit Rhizobien beimpften als auch bei den unbeimpften Pflanzen (Tab. 111u.112). Hier konnten die Rhizobien die toxische Wirkung des Blei nicht wesentlich mindern.

Frankia konnte die Pflanzen ebenfalls nicht gegen die schädigende Wirkung von $\mathrm{Pb}$ unterstützen. Die beimpften Pflanzen wuchsen stets schlechter als die unbeimpften (Tab.120). Fabig (1982) berichtete über eine schädigende Wirkung des $\mathrm{Pb}$ auf die Symbiose mit (V)AMykorrhiza-Pilzen bei verschiedenen Pflanzenarten.

Einfluss der Schwermetall-Behandlung auf die Aufnahme von N, P, K, Ca und $\mathrm{Mg}$

Die Schwermetall-Wirkung auf die Konzentration und Aufnahme der Nährstoffe hing nicht nur von den Baumarten ab, sondern war manchmal elementspezifisch.

Einfluss des Zn

Die Ergebnisse zeigten, dass die Konzentration von N, P, K, Ca und Mg in den Sprossen von A. saligna mit steigenden Zn-Zugabe absank (Tab.10 u.11). In den Sprossen von C. equisetifolia war dies ähnlich mit Ausnahme der Ca und Mg-Konzentration (Tab.31 u. 32). Hingegen stieg die Konzentration von $\mathrm{N}, \mathrm{P}, \mathrm{K}, \mathrm{Ca}$ und $\mathrm{Mg}$ in den Sprossen von C. sempervirens an (Tab.52 u.53).

Die Aufnahme von allen Nährstoffen $\mathrm{N}, \mathrm{P}, \mathrm{K}, \mathrm{Ca}$ und $\mathrm{Mg}$ wurde in den Sprossen von A. saligna mit zunehmender Zn-Gabe wegen des verbesserten Wachstums erhöht (Tab.10 u. 11). Die Nährstoffaufnahme im Spross von $C$ equisetfolia nahm mit Ausnahme von $\mathrm{K}$ und $\mathrm{Mg}$ ab (Tab.32). Im Spross von C. sempervirens stieg die $\mathrm{N}$, K- und Mg-Aufnahme an, während bei $\mathrm{P}$ und $\mathrm{Ca}$ eine Herabsetzung der Aufnahme zu verzeichnen war (Tab.52 u.53). Schoemakers (1986) berichtete, dass eine hohe Zn-Behandlung zu einer Verringerung der PAufnahme in den Wurzeln, Blättern und Früchten führte. Auch Foroughi et al. (1979) fanden, dass durch die Schwermetallaufnahme der P-und K-Gehalt der Pflanzen negativ beeinflusst wurde.

Darüber hinaus führte die Beimpfung mit Rhizobien bei A. saligna oder mit Frankia bei C. equisetifolia bei steigenden Zn-Konzentrationen im Boden $\mathrm{zu}$ einer Verringerung der Konzentrationen von N, P, K, Ca und Mg. Die Konzentrationen der Nährstoffe N, P, K, Ca und $\mathrm{Mg}$ der beimpften Acacia-Pflanzen waren niedriger als bei den unbeimpften Pflanzen (Tab.99 u. 100). Anders war es jedoch im Spross der Casuarina-Pflanzen. Die 
Konzentrationen von $\mathrm{N}, \mathrm{P}, \mathrm{K}, \mathrm{Ca}$ und $\mathrm{Mg}$ der beimpften Casuarina-Pflanzen lagen etwas höher oder ebenso hoch wie bei den unbeimpften Pflanzen (Tab. 124 u.127).

Die Aufnahme von N, P, K, Ca und Mg in den Sprossen der beimpften Pflanzen (A saligna mit Rhizobien oder C. equisetifolia mit Frankia) und der unbeimpften Pflanzen wurde durch steigende Zn-Gaben erhöht.

Die beimpften Acacia-Pflanzen wiesen höhere Aufnahme an N, P, K, Ca und $\mathrm{Mg}$ gegenüber den unbeimpften Pflanzen auf. Bei C. equisetifolia war die Aufnahme dieser Nährstoffe der beimpften Pflanzen jedoch niedriger als die der unbeimpften Pflanzen.

\section{Einfluss des Cd}

Die Behandlung mit Cd rief eine Verringerung der Konzentrationen von $\mathrm{N}, \mathrm{P}, \mathrm{K}, \mathrm{Ca}$ und $\mathrm{Mg}$ im Spross von A. saligna, von N, P, K, und Mg im Spross von C. equisetifolia und von N, $\mathrm{Ca}$ und $\mathrm{Mg}$ im Spross von C. sempervirens hervor (Tab. 17-18, 38-39 u.59-60). Zu ähnlichen Ergebnissen kamen Arduini et al. (1998). Sie berichteten, dass die Behandlung mit 1 oder $5 \mu \mathrm{M} \mathrm{Cd}$ in Abhängigkeit von der Baumart zu einer Abnahme des Ca- und $\mathrm{Mg}$ Gehaltes in den Blättern führte. Eine Verringerung des Mg-Gehaltes durch Cadmium fanden auch Walker et al. (1977), Khan und Khan (1983), Greger und Lindberg (1987) sowie Hagemeyer et al. (1994). Barcelo et al. (1985) stellten bei Bohnen fest, dass der Mg-Gehalt in den jungen Blättern ab der 4. und 5. Versuchswoche bei 40 bzw. 20 ppm Cd und ab 6 . Woche für 1 und 10 ppm Cd im Boden abnahm. Nach Kabata-Pendias und Pendias (1984) liegt die Vermutung nahe, dass $\mathrm{Cd}$ im allgemeinen den Gehalt an $\mathrm{Ca}, \mathrm{P}$ und $\mathrm{K}$ der Pflanzen verringert.

Unter dem Einfluss steigender Cd-Konzentrationen im Boden führte die Beimpfung der Acacia-Pflanzen mit Rhizobien zu einer Herabsetzung der Konzentrationen von N, P, K, Ca und $\mathrm{Mg}$ in den Sprossen, obwohl die Aufnahme der Elemente in den Sprossen dieser Pflanzen wegen des verbesserten Wachstum deutlich höher als in den unbeimpften Pflanzen lag. (Tab.106 u. 107). Nicht nur die Symbiose mit Rhizobien bei den Acacia-Pflanzen, sondern auch die Symbiose mit den (V)A-Mykorrhiza-Pilzen verursachte eine Erhöhung der PAufnahme in den Sprossen verschiedener Pflanzenarten bei zunehmenden Cd-Gaben (Fabig, 1982).

Darüber hinaus sank die Aufnahme der erwähnten Nährstoffe im Spross der mit Frankia beimpften Casuarina-Pflanzen bei steigenden Cd-Gaben zum Boden wegen der Beeinträchtigung des Wachstums ab (Tab. 125 u. 128). Vergleichbare Angaben sind in der Literatur nicht vorhanden. 


\section{Einfluss des $\mathrm{Pb}$}

Unter Einfluss steigender Pb-Zugaben zum Boden erhöhte sich, mit Ausnahme der CaKonzentration im Spross der Casuarina-Pflanzen, die Konzentration der anderen Nährstoffe im Spross aller Baumarten (Tab. 24-25, 45-46 u. 66-67). Im zweiten Versuch wiesen die Pflanzen der drei Baumarten unter Abzug des im verwendetem Bleinitrat enthaltenden Stickstoffs bei steigender Pb-Applikation ähnliche Tendenzen der Nährstoff-Konzentrationen bzw. -Aufnahmen wie im ersten Versuch auf. In anderen Worten, der Stickstoff des Bleinitrats hatte bei der Steigerung dieses Salzes keinen Einfluss auf das Wachstum und die Nährstoffaufnahme der untersuchten Nährstoffe N, P, K, Ca und Mg.

Wie im ersten und zweiten Versuch zeigten die beimpften Acacia- (mit Rhizobien) und Casuarina- (mit Frankia) Pflanzen im dritten Versuch ein ähnliches Bild hinsichtlich der Nährstoffkonzentration bzw. -Aufnahme. Wie schon erwähnt, direkt vergleichbare Untersuchungen sind in der Literatur kaum vorhanden. Lediglich Fabig (1982) und Goergen (1987) fanden erhöhte Aufnahmen verschiedener Nährstoffe bei unterschiedlichen Pflanzenarten unter dem Einfluss zunehmender Pb-Zufuhr zum Boden, wenn die Pflanzen mit (V)A-Mykorrhiza-Pilzen beimpft waren.

Im allgemeinen konnten die Konzentrationen und Aufnahmen der Nährstoffe bei steigender Schwermetall-Behandlung mit $\mathrm{Zn}$, $\mathrm{Cd}$ oder $\mathrm{Pb}$ nicht mit den positiven oder negativen Effekten solcher Behandlungen auf das Pflanzenwachstum direkt korreliert werden. Eine Ausnahme macht eventuell der Stickstoff im dritten Versuch bei den mit Rhizobien beimpften Acacia-Pflanzen.

Konzentration und Aufnahme der Schwermetalle $\mathrm{Zn}, \mathrm{Cd}$ und $\mathrm{Pb}$ in den Sprossen und Wurzeln

\section{Zink}

In den vorliegenden Untersuchungen (Versuch 1) wurde die Konzentration bzw. Aufnahme des $\mathrm{Zn}$ in den Sprossen und insbesondere in den Wurzeln der drei Baumarten in allen Versuchen durch steigende Zn-Zufuhr zum Boden drastisch erhöht. Trotz stark erhöhter ZnAufnahme produzierten die Acacia- und Cupressus-Pflanzen die höchsten STG und WTG bei Zn-Zugabe bis $10 \mathrm{mg} / \mathrm{kg}$ Boden. Bei den Casuarina-Pflanzen war dies bei einem weiten Spektrum der Zn-Behandlung bis $20 \mathrm{mg} / \mathrm{kg}$ Boden der Fall. Die ausgeprägte Toleranz der Casuarina-Pflanzen gegen hohe Zn-Gehalte im Boden wird auch deutlich, wenn das Wachstum bei der höchsten Zn-Behandlung im Verhältnis zum höchsten Wachstum betrachtet wird. Bei den Casuarina-Pflanzen betrug dies $86 \%$ gegenüber $58 \%$ bei den Pflanzen der andern beiden Baumarten (Tab. 72). 
Die mit Rhizobien beimpften Acacia-Pflanzen nahmen größere Mengen an Zn aus dem Boden auf als die unbeimpften Pflanzen. Jedoch haben die beimpften und nicht beimpften dem Boden bei weitem viel mehr $\mathrm{Zn}$ entzogen als die unbehandelten Kontrollpflanzen. Obwohl die Beimpfung der Casuarina-Pflanzen mit Frankia zu höheren Zn-Konzentrationen im Spross bei steigender Zn-Zugabe zum Boden führte, bleibt die Rolle der Frankia in diesem Zusammenhang noch unklar. Unter den Versuchsbedingungen wirkte die Frankia-Beimpfung negativ auf das Wachstum. Direkt vergleichbare Untersuchungen sind kaum vorhanden. Fabig (1982) und Franz und Werner (1995) stellten höhere Zn-Gehalte in den Wurzeln von Pflanzen fest, die mit (V)A-Mykorrhiza-Pilzen inokuliert waren und in mit Zn kontaminierten Böden wuchsen.

\section{Cadmium}

Von großer Bedeutung war die positive Wirkung der niedrigen Konzentrationen des bekanntlich toxischen $\mathrm{Cd}$ auf das Wachstum der Pflanzen (Tab.71). Die Acacia-Pflanzen waren am tolerantesten, die STG und WTG stiegen bei Zugabe von 5 ppm Cd höchst signifikant gegenüber der Kontrolle. Bei den Casuarina- und Cupressus-Pflanzen trat dieses Phänomen schon bei der niedrigeren Cd-Gabe von $2,5 \mathrm{mg} / \mathrm{kg}$ Boden auf, jedoch nicht so deutlich wie bei A. saligna, was auch durch die Ergebnisse des dritten Versuches bestätigt wurde. Hier wiesen insbesondere die mit Rhizobien beimpften Acacia-Pflanzen viel höhere STG und WTG bei Cd-Zugabe bis $5 \mathrm{mg} / \mathrm{kg}$ Boden auf (Tab.104 u. 105). Die mit Frankia beimpften Casuarina-Pflanzen akkumulierten große Cd-Mengen in ihren Wurzeln. Bemerkenswert war weiterhin die Fähigkeit der Casuarina-Pflanzen, ein starkes Wurzelsystem bei Cd-Gaben bis $10 \mathrm{mg} / \mathrm{kg}$ Boden zu bilden. Darüber hinaus ergab sich bei den Cupressus-Pflanzen ein Verhältnis des STG bei der höchsten Cd-Gabe zum höchsten bzw. optimalen STG von $42 \%$, gegenüber $33 \%$ bei Acacia und $25 \%$ bei den CasuarinaPflanzen (Tab.72).

Im allgemeinen stiegen die Konzentrationen und Aufnahmen der Nährstoffe bei den drei Baumarten in allen Versuchen mit steigender Cd-Konzentration im Boden stark an. Die Wurzeln akkumulierten viel mehr $\mathrm{Cd}$ als die Sprosse. $\mathrm{Zu}$ ähnlichen Ergebnissen sind John (1973), Jarvis et al. (1976), Costa und Spitz (1997) und Mazen und El Maghraby (1997/98) gekommen. Kloke und Schenke (1979) berichteten über unterschiedliche Empfindlichkeiten verschiedener Pflanzenarten gegenüber Cd. Fabig (1982) stellte verschiedene Reaktionen von Capsicum- und Chromolaena-Pflanzen auf $\mathrm{Cd}$ im Boden fest. Nach Luis et al. (1998) akkumulierten die Pflanzen $90 \%$ des aufgenommenen $\mathrm{Cd}$ in ihren 
Wurzeln. Moral et al. (1994) und Franz und Werner (1995) erhielten ebenfalls ähnliche Ergebnisse.

Blei

Mit steigender $\mathrm{Pb}$-Konzentration im Boden nahmen die P-Konzentrationen und -Aufnahmen in den Sprossen und Wurzeln der Pflanzen der drei Baumarten nicht nur in Versuch 1 und 2 sondern auch im dritten Versuch bei den mit Rhizobien beimpften Acacia- und mit Frankia beimpften Casuarina-Pflanzen zu (Tab.71 u. 140). Die Cupressus-Pflanzen zeigten eine höhere Toleranz gegenüber Pb und produzierten die höchsten STG und WTG bei Pb-Zugabe von $20 \mathrm{mg} / \mathrm{kg}$ Boden, während die Pflanzen der anderen beiden Baumarten ein verbessertes Wachstum nur bei $\mathrm{Pb}$-Gaben bis $10 \mathrm{mg} / \mathrm{kg}$ Boden erzielten. Dieses Verhalten zeigte sich auch beim Verhältnis des TG bei höchster Pb-Gabe zum höchsten bzw. optimalen TG (Tab.72). Bei Cupressus lag das Verhältnis bei $86 \%$ gegenüber $71 \%$ bei Acacia und $78 \%$ bei C. equisetifolia. Wie beim $\mathrm{Cd}$ lag die $\mathrm{Pb}$-Aufnahme der Wurzeln der drei Baumarten allgemein viel höher als in den Sprossen. Über ähnliche Befunde wurde auch von Fabig (1982) und Goergen (1987) berichtet.

Einfluss der Beimpfung mit $\mathrm{N}_{2}$-fixierenden Bakterien

\section{Beimpfung mit Rhizobien}

In den vorliegenden Untersuchungen führte die Beimpfung der Acacia-Pflanzen bei den niedrigen und mittleren Stufen von $\mathrm{Zn}, \mathrm{Cd}$ und $\mathrm{Pb}$ zu einer Steigerung des Wachstums. Bei der höchsten Stufe aller Schwermetalle, besonders des Cd, sank das Wachstum jedoch wieder ab. Baszynski et al. (1980) fanden, dass der $\mathrm{Mg}$-Gehalt durch $\mathrm{Cd}$-Behandlung verringert wurde und somit auch der Chlorophyllgehalt, was eine Beeinträchtigung des Wachstums verursachte. In diesen Untersuchungen scheint eine Verringerung der Boden-pH-Werte während der Versuche, eine Rolle gespielt zu hohen. Die $\mathrm{pH}$-Werte im Boden der unbeimpften Acacia-Pflanzen waren bei allen Schwermetall-Behandlungen etwas höher als im Boden der beimpften Pflanzen. Aguilars und Van Diest (1981) deuteten daraufhin, dass besonders die Leguminosenwurzeln hohe Mengen an $\mathrm{H}^{+}$in die Rhizosphäre abscheiden. Diese $\mathrm{H}^{+}$-Abscheidung der Leguminosenwurzeln hängt ursächlich mit der symbiotischen $\mathrm{N}_{2^{-}}$ Fixierung zusammen (Mengel und Steffens, 1982). Das schlechtere Wachstum der Leguminosen bei steigender Bodenacidität kann zum einen an einer direkten Hemmung des Pflanzenwachstums liegen und zum anderen an einer negativen Beeinflussung der Rhizobien und/oder der Knöllchenbildung an einer Erhöhung der Aufnahme von Schadelementen (wie

Schwermetalle und $\mathrm{Al}$ ). Bekanntlich hängt die Verfügbarkeit bzw. Aufnahme der Schwermetalle stark vom Boden-pH ab. 
Von großer Bedeutung war die vorher erwähnte positive Wirkung der niedrigen und mittleren Gaben von $\mathrm{Zn}, \mathrm{Cd}$ und $\mathrm{Pb}$ auf das Wachstum der beimpften Acacia-Pflanzen. Dieser Befund kann für die Praxis von großer Relevanz sein.

Die Stickstoffaufnahme u.a. (?) stieg in den beimpften Acacia-Pflanzen bei den genannten Schwermetall-Gaben stark an. Hier erhebt sich die Frage, ob der Hauptgrund des positiven Effekts der Rhizobien-Beimpfung in der $\mathrm{N}_{2}$-Fixierung bzw. in der verbesserten N-Vorsorgung der Pflanzen lag und inwieweit hohe Konzentrationen von Schwermetallen im Boden diesen Prozess beeinflussen? Zur Klärung dieser Fragen bedarf es noch intensiver Untersuchungen.

\section{Beimpfung mit Frankia}

Im dritten Versuch konnte die Beimpfung der Casuarina-Pflanzen mit Frankia das Wachstum nicht fördern. Das Wachstum der beimpften Pflanzen wurde sogar bei allen Schwermetall-Behandlungen beeinträchtigt im Vergleich $\mathrm{zu}$ den unbeimpften Pflanzen. Bekanntlich zeigen die Actinomyceten (Frankia) bei Casuarina ihre höchste Effizienz der $\mathrm{N}_{2}$-Fixierung meistens, wenn die Wirtspflanzen in alkalischen bzw. kalkhaltigen Böden wachsen. Deshalb sind die Casuarina-Pflanzen hauptsächlich in den ariden und semiariden Gebieten verbreitet, wo solche Böden häufig vorkommen (Diem und Dommergues, 1990). Die Messung des Boden-pH-Wertes am Ende jedes Versuches der vorliegenden Arbeit zeigte eine deutliche Absenkung des Ausgangs-pH-Wert des Bodens der beimpften und unbeimpften Pflanzen während der Versuche. Es wird vermutet, dass diese Versauerung des Bodens die Effizienz der symbiotischen Frankia hemmte, so dass diese Bakterien wegen ihres Bedarfes an Assimilaten das Wachstum der beimpften Pflanzen schwächten, obwohl sie Knöllchen an den Wurzeln gebildet hatten.

\section{Die $\mathrm{pH}-\mathrm{Werte}$ des Bodens}

Der Ausgangs-pH-Wert des Bodens aller Versuche lag bei 7. Im Verlauf der Vegetationsperioden verringerte sich das Boden-pH um manchmal mehr als $2 \mathrm{pH}$ bis zum Ende der Versuche. Für eine präzise Interpretation der Wirkung des Boden-pH auf das Wachstum der Pflanzen (besonders bei C. equisetifolia) unter Einfluss der Schwermetall-Behandlung fehlen Messungen der Boden-pH-Werte in Intervallen während der Vegetationsperiode jedes Versuches. Die Notwendigkeit solcher Messungen wurde besonders im dritten Versuch (besonders nach Frankia-Beimpfung) deutlich. In den vorliegenden Untersuchungen musste jedoch jeder der drei Versuche mit dem gleichen Boden bzw. bei gleichem Boden-pH durchgeführt werden, um den Vergleich zwischen den Resultaten der drei Versuche zu gewährleisten. 


\subsection{Schlussfolgerung}

Die Ergebnisse der vorliegenden Arbeit demonstrieren die unterschiedliche Reaktion der drei Baumarten Acacia saligna, Casuarina equisetifolia und Cupressus sempervirens auf steigende Konzentrationen der im Rahmen des Umweltschutzes wichtigsten Schwermetalle $\mathrm{Zn}, \mathrm{Cd}$ und $\mathrm{Pb}$ im Boden. Zum Beispiel wiesen die Casuarina-Pflanzen eine deutliche Toleranz gegen höhere Zn-Konzentrationen im Boden auf als die anderen beiden Bäume. Gegen hohe Cd-Konzentrationen waren die Acacia-Pflanzen toleranter als die Casuarina- und Cupressus-Pflanzen.

Beim $\mathrm{Pb}$ reagierten die Casuarina-Pflanzen empfindlicher als die anderen beiden Baumarten. Dies zeigt, dass die Artwahl eine entscheidende Rolle beim Anbau von holzlieferenden Bäumen auf Schwermetall kontaminierten Böden spielt. Die Eignung einer Baumart für den Anbau auf solchen Böden muss in Voruntersuchungen ermittelt werden, um den Erfolg solcher Maßnahme zu sichern.

Die Feststellung der vorliegenden Untersuchung, dass solche holzlieferenden Bäume nicht nur hohe Schwermetall-Konzentrationen im Boden tolerieren, sondern auch große Mengen dieser Schwermetalle dem Boden entziehen können, gibt Anlass für die Forcierung des Anbaus solcher Bäume zur Begrünung bzw. Rekultivierung von kontaminierten Böden als Umweltschutzmaßnahme und gleichzeitig als Beitrag zur Steigerung der Produktion von Nutzholz in Ägypten. Die Befunde aus Gefäßversuchen unter Gewächshausbedingungen bedürfen jedoch noch der Bestätigung unter praktischen Bedingungen in den kontaminierten Gebieten Ägyptens.

Darüber hinaus zeigten die Ergebnisse, dass das Potential der in Gefäßversuchen unter Gewächshausbedingungen untersuchten Baumarten noch nicht erschöpft ist, zumal die Pflanzen hier mit steigender Schwermetall-Konzentration im Boden entweder besser wuchsen (sogar beim bekanntlich toxischen $\mathrm{Cd}$ ), oder doch nur wenig durch die getesteten höchsten Schwermetall-Gaben geschädigt wurden. Dies deutet darauf hin, dass noch ein höheres Potential der Toleranz gegen die Schwermetalle in den Baumarten stecken könnte.

Ein weiteres Potential der Toleranz liegt in der Nutzung der biologischen Fähigkeiten der Baumarten verschiedener Familien. Die Ergebnisse machen deutlich, wie positiv eine Beimpfung mit den $\mathrm{N}_{2}$-fixierenden Rhizobien auf das Wachstum bzw. die Toleranz von A. saligna als Leguminose wirkte, wenn diese Pflanzen auf kontaminierten Böden wuchsen. Obwohl die Beimpfung der Casuarina-Pflanzen mit den aktiven $\mathrm{N}_{2}$-fixierenden Bakterien der Actinomyceten (Frankia) das Wachstum der Pflanzen im dritten Versuch nicht förderte, könnte in einer solchen biologischen Maßnahme ein großes Potential für die Praxis gegeben sein. Dies setzt jedoch noch gezielte Untersuchungen zur Optimierung der Effizienz einer 
Beimpfung mit Frankia voraus. In diesem Zusammenhang müssen die Wechselbeziehungen zwischen Baumarten, Frankia-Arten und Bodeneigenschaften, insbesondere das Boden-pH, näher untersucht werden.

Nicht zuletzt sollte die biologische Fähigkeit der Baumarten genutzt werden, eine Symbiose mit den (V)A-Mykorrhiza-Pilzen einzugehen. Durch langjährige Erfahrungen an unserem Institut ist zu erwarten, dass die Inokulation der Bäume mit solchen symbiotischen Pilzen zur Verbesserung des Wachstums dieser Bäume durch verbesserte Nährstoffaufnahme (in erster Linie des P) und zur Erhöhung ihrer Toleranz gegen die Schwermetalle in den Böden beitragen wird.

Die vorliegende Arbeit stellt einen ersten Anstoß zur Erforschung des Problems den mit Schwermetallen kontaminierten Böden Ägyptens bzw. einen Beitrag zum Umweltschutz in diesem Lande dar. 


\section{Zusammenfassung}

In drei Gefäßversuchen unter Gewächshausbedingungen wurde der Einfluss steigender Zugaben der Schwermetalle $\mathrm{Zn}$ (als $\mathrm{ZnSO}_{4} .7 \mathrm{H}_{2} \mathrm{O}$ ), $\mathrm{Cd}$ (als $\mathrm{CdSO}_{4} .8 \mathrm{H}_{2} \mathrm{O}$ ) und $\mathrm{Pb}$ (als $\left.\mathrm{Pb}\left(\mathrm{NO}_{3}\right)_{2}\right)$ zum Boden auf das Wachstum und Nährstoffaufnahme der drei holzliefernden Baumarten Acacia saligna, Casuarina equisetifolia und Cupressus sempervirens untersucht.

In allen Versuchen wurde eine Bodenmischung aus Schoninger-Boden (ein nährstoffarmer sandiger mit $\mathrm{CaCO}_{3}$ auf $\mathrm{pH} 7$ aufgekalkter Boden) und Quarzsand im Verhältnis 2:1 verwendet.

Aus Ägypten stammende Samen wurden in Anzuchtschalen gesät. Nach zweimonatiger Anzuchtperiode wurden die Sämlinge in $1 / 3 \mathrm{~kg}$ Töpfe umgepflanzt. Nach weiteren 4 Monaten wurden die Pflanzen in $2 \mathrm{~kg}$ Töpfe umgepflanzt.

Die wichtigsten Resultate waren:

Versuch 1

- Generell erhöhte die Behandlung mit den Schwermetallen $\mathrm{Zn}, \mathrm{Cd}$ und $\mathrm{Pb}$ das Wachstum (Spross und Wurzel) der drei Baumarten: mit Zn bis zu höchsten Stufe $40 \mathrm{mg} / \mathrm{kg}$ Boden bei A. saligna, $20 \mathrm{mg} / \mathrm{kg}$ Boden bei C. equisetifolia und $10 \mathrm{mg} / \mathrm{kg}$ Boden bei C. sempervirens); mit Cd bis zur mittleren Stufe $5 \mathrm{mg} / \mathrm{kg}$ Boden bei $A$ saligna und bis zur Stufe 2,5 mg/kg Boden bei C. equisetifolia und C. sempervirens und mit $\mathrm{Pb}$ bis zur Stufe $20 \mathrm{mg} / \mathrm{kg}$ Boden bei A. saligna und $40 \mathrm{mg} / \mathrm{kg}$ Boden bei C. equisetifolia und C. sempervirens.

- Im allgemeinen waren die Casuarina-Pflanzen toleranter gegen höhere Zn-Konzentrationen im Boden als die anderen beiden Baumarten. Gegen höhere Cd-Konzentrationen waren die Acacia-Pflanzen toleranter. Von größter Bedeutung war die positive Wirkung einer Zugabe von Cd bis 5mg/kg Boden auf das Wachstum der Pflanzen.

- Die Konzentration bzw. Aufnahme der Nährstoff N, P, K, Ca und Mg war bei steigender Behandlung mit $\mathrm{Zn}, \mathrm{Cd}$ und $\mathrm{Pb}$ unterschiedlich bei den drei Baumarten.

- Bemerkenswert war die verringerte Mg-Konzentration bzw. -Aufnahme der drei Baumarten durch zunehmende Cd-Konzentration im Boden.

- Bei steigenden Zugaben von $\mathrm{Zn}, \mathrm{Cd}$ und $\mathrm{Pb}$ zum Boden wurden die Konzentrationen und Aufnahmen der drei Schwermetalle in den Sprossen und Wurzeln aller Pflanzen stark erhöht. Die Werte der Wurzeln lagen meistes höher als die der Sprosse. 


\section{Versuch 2}

In diesem Versuch wurde eine eventuelle Nebenwirkung des im verwendetem Bleinitrat vorhandenen $\mathrm{N}$ auf das Verhalten der Pflanzen gegenüber steigender Konzentrationen an $\mathrm{Pb}$ im Boden ausgeschlossen. Hierfür wurde die N-Menge des Bleinitrats von der vorgesehenen N-Düngungsmenge abgezogen.

- Die Ergebnisse zeigten, dass die N-Menge im Bleinitrat keinen Einfluss auf die Reaktion der Pflanzen bei zunehmender Schwermetall-Konzentration im Boden hatte. Abgesehen von unbedeutenden Unterschieden wiesen die Ergebnisse ähnliche Tendenzen wie im Versuch 1 auf.

\section{Versuch 3}

In diesem Versuch wurde die Wirkung der Beimpfung mit den $\mathrm{N}_{2}$-fixierenden Bakterien Rhizobien bei Acacia saligna und Frankia bei Casuarina equisetifolia auf das Wachstum und die Nährstoffaufnahme dieser Pflanzen bei steigenden Konzentrationen von $\mathrm{Zn}, \mathrm{Cd}$ und $\mathrm{Pb}$ im Boden geprüft.

- Die Ergebnisse zeigen, dass die Beimpfung der Acacia-Pflanzen mit Rhizobien die TSBildung sowohl in den Sprossen als auch in den Wurzeln bei allen Schwermetallbehandlungen im Vergleich zu den nicht beimpften Pflanzen förderte.

- Die Konzentration der Nährstoffe N, P, K, Ca und Mg waren in den beimpften Pflanzen niedriger als in den unbeimpften (Verdünnungseffekt). Entsprechend dem verbesserten Wachstum lag die Aufnahmen dieser Nährstoffe in den beimpften Pflanzen höher als in den unbeimpften (mit Ausnahme der Pb-Behandlung). Gleiche Tendenzen der Konzentration und Aufnahme der Schwermetalle $\mathrm{Zn}, \mathrm{Cd}$ und $\mathrm{Pb}$ in den beimpften und unbeimpften Pflanzen wurden ebenfalls festgestellt. Jedoch wurden die Schwermetalle viel stärker aufgenommen, besonders bei den unbeimpften Pflanzen.

- Die Beimpfung der Casuarina-Pflanzen mit Frankia konnte das Wachstum der Pflanzen nicht fördern. Das Sprosstrockengewicht der unbeimpften Casuarina-Pflanzen lag höher bei den $\mathrm{Zn}$ - und $\mathrm{Cd}$ - Behandlung und gleich hoch bei der Pb-Behandlung verglichen mit den beimpften Pflanzen. Hingegen lagen die Wurzeltrockengewichte der beimpften Pflanzen bei allen Schwermetall-Behandlungen, vermutlich wegen der Knöllchenbildung, höher als bei den unbeimpften Casuarina-Pflanzen.

- Die Konzentrationen und Aufnahmen von N, P, K, Ca und Mg in den Sprossen zeigten keine klaren Tendenzen.

- Wie in Versuch 1 wurde die Konzentration und Aufnahme von $\mathrm{Zn}, \mathrm{Cd}$ und Pb im Spross der beimpften und unbeimpften Pflanzen durch steigendende Zugabe zum Boden allgemein stark erhöht. Die Wurzeln ließen gegenüber dem Spross ein ähnliches Bild erkennen. 
- Bemerkenswert war die Absenkung des Ausgangs-pH des Versuchsbodens am Ende jedes Versuches 


\section{LITERATUR}

AGUILARS, A. and DIEST, A. VAN, 1981: Rock phosphate mobilization induced by the alkaline uptake pattern of legume utilizing symbiotically fixed nitrogen. Plant and Soil 61, 2742 .

ANDERSON, A. and NILSSON, K.O., 1974: Influence of lime and soil pH on Cd availability to plants. Ambio 3, 198-200.

ARDUINI, I., GODBOLD, D.L., ONNIS, A. and STEFANI, A., 1998: Heavy metals influence mineral nutrition of (tree) seedlings. Chemosphere 36, 739-744.

ARORA, J.S. and SINGH, J.R., 1970: Some effect of iron spray on growth, yield and quality of guava trees (Psidium guava L.). J.Jap.Soc.Hort.Sci., 39, 139-143. (c.f. Agric.Res.Rev. (1981), 59 No.3)

ARRIECHI, E. and RAMIREZ, R., 1997: Soil test for available zinc in acid soils of Venezuela. Comm.Soil Sci.Plant Anal. 28, 17-18.

ASPIRAS, R.B., 1981: Nitrogen fixation in nodulated non-legumes growing in the Philippines. Canopy Intern. 7(7), 3-5.

ATEN, C.F. and GUPTA, S.K., 1996: On heavy metals in soil; rationalization of extractions by dilute salt solutions, comparison of the extracted concentrations with uptake by ryegrass and lettuce, and the possible influence of pyrophosphate on plant uptake. Sci.Total Environ. $178,45-53$.

BADRAN, O.A. and KANDEEL, E.E., 1971: Principles of Timber Trees, Wood Science and Technology. Dar El-Maaref, Egypt.

BADRI, M. and SPRINGUEL, I., 1994: Biogeochemical prospecting in the South Eastern Desert of Egypt. J.Arid Environ. 28, 257-264.

BARCELO, J., POSCHENRIEDER, CH. u. CABOT, C., 1985: Cadmium-, Mangan-, EisenZink- und Magnesium-Gehalte von Bohnenpflanzen (Phaseolus vulgaris L.) in Abhängigkeit von Dauer und Höhe des Cadmium-Angebotes. Z. Pflanzenern.Bodenk .148, 278-288. 
BASZYNSKI, T., WAJDA, L., KROL, M., WOLINSKA, D., KRUPA, Z. and TUKENDORF, A., 1980: Photosynthetic activities of Cd treated tomato plants. Physiol.Plant. $48,365-370$.

BRÜNE, H., ELLINGHAUS, R. und HEYN, J., 1982: Schwermetallgehalte hessischer Böden und ergänzende Untersuchungen zur Schwermetallaufnahme durch Pflanzen. Kali-Briefe (Büntehof) 16(5), 271-291.

BUCHAUER, M.J., 1973: Contamination of soil and vegetation near a zinc smelter by zinc, cadmium, copper and lead. Environ.Sci.Technol. 7, 131-135.

CHUAN, M.C., SHU, G.Y. and LIU, J.C., 1996: Solubility of heavy metals in a contaminated soil: effects of redox potential and pH. Water Air Soil Pollution 90, 543-556.

CIESLINSKI, G., VAN REES, K.C.J., HUANG, P.M., KOZAK, L.M., ROSTAD, H.P.W and KNOTT, D.R, 1996: Cadmium uptake and bioaccumulation in selected cultivars of durum wheat and flax as affected by soil type. Plant and Soil 182, 115-124.

COSTA, G. and SPITZ, E., 1997: Influence of cadmium on soluble carbohydrates, free amino acids, and? protein content of in vitro cultured Lupinus albus. Plant Sci. 128, 131-140.

CRÖSSMANN, G., 1982: Kontamination Pflanzlicher Lebensmittel, insbesondere mit Schwermetallen. Landwirtsch.Forsch., Sonderh. 38, Kongressband Trier, 608-615

DE LAJUdIE, P., WILlEMS, A., POT, B., DEWETTINCK, D., MAESTROJUAN, G., NEYRA, M., COLLINS, M.D., DREYFUS, B., KERSTERS, K. and GILLIS, M., 1994: Polyphasic taxonomy of rhizobia: emendation of the genus Sinorhizobium and description of Sinorhizobium meliloti comb. nov., Sinorhizobium saheli sp. nov., and Sinorhizobium teranga sp. nov. Int.J.Syst.Bacteriol. 44, 715-733.

DIEM, H.G. und DOMMERGUES, Y.R., 1990: Current and potential uses and management of Casuarinaceae in the tropics and subtropics. In: Ch.R.Schintzer and Tjepkema, J.D. The Biology of Frankia and Actinorhizal plants. Academic Press, London. 
DSMZ. 1983: (Deutsche Sammlung von Mikroorganismen und Zellkulturen GmbH), Catalogue of Strains, Mascheroder Weg 1b, 38124 Braunschweig, Deutschland.

DSMZ. 1993: (Deutsche Sammlung von Mikroorganismen und Zellkulturen GmbH), Catalogue of Strains, Mascheroder Weg 1b, 38124 Braunschweig, Deutschland.

DUDKA, S., PONCE-HERNANDEZ, R., TATE, G. and HUTCHINSON, T.C., 1996: Forms of $\mathrm{Cu}, \mathrm{Ni}$ and $\mathrm{Zn}$ in soils of Sudbury, Ontario and he metal concentrations in plants. Water Air Soil Pollution 90, 531-542

EL-BEHEIDI, M., 1972: Effect of some microelements on the growth and seed yield of cucumber. Res.Bull., Ain Shams Univ., Fac. of Agric., Egypt.

EL-GHAMRINY, E.A., 1976: Response of Cucumber Plants to Some Microelements. M.Sc.Thesis, Fac. of Agric., Zagazig Univ., Egypt.

EL-HADIDY, Z.A., FRANCES, R.R. and SHEHATA, M.A., 1980: Effect of spraying with some microelements on the pigments in wheat blades, yield and carbohydrate fractions of grains. Res.Bull., Ain Shams Univ., Fac. of Agric., Egypt.

EL-SAIED, Z.M., ABDALLA, I.M., HELAL, R.M. and AMER, A.S., 1981: Preliminary investigation on improving yield and quality of tomato by aid of micronutrients foliar sprays. Res.Bull., Ain Shams Univ., Fac. of Agric., Egypt.

ERLENKEUSER, H., SUESS, E. and WILLKOMM, H., 1974: Industrialization affects heavy metal and carbon isotope concentration in recent Baltic sea sediments. Geochim.Cosmoch im. Acta 38, 823-824

ERNST, W., 1974: Schwermetallvegetation der Erde. Fischer, Stuttgart.

FABIG, B., 1982: Einfluß von $\mathrm{Al}$ und den Schwermetallen $\mathrm{Fe}, \mathrm{Mn}, \mathrm{Zn}, \mathrm{Cu}, \mathrm{Pb}$ und $\mathrm{Cd}$ auf die Effizienz der VA-Mykorrhiza bei tropischen und subtropischen Pflanzen. Dissertation, Göttingen.

FRANCESCHI, V.R. and SCHUERN, A. M., 1986: Incorporation of strontium into plant calcium oxalate crystals. Protoplasma 130: 199-205 
FOROUGHI, M., HOFFMANN, G., TEICHER, K. und VENTER, F., 1976: Der Einfluß unterschiedlich hoher Gaben von Cadmium, Chrom oder Nickel auf Tomaten in Nährlösung. Landwirtsch.Forsch., Sonderh. 32, Kongressband Mannheim, 37-48.

FOROUGHI, M., TEICHER, K. und VENTER, F., 1979: Die Wirkung steigender Gaben von Blei, Cadmium, Nickel oder Zink auf Spinat in Nährlösung. Landwirtsch.Forsch., Sonderh. 35, Kongressband Augsburg, 599-606.

FOROUGHI, M., VENTER, F. und TEICHER, K., 1981: Wirkung von steigenden MüllKlärschlamm-Kompost-Gaben auf den Schwermetallgehalt von Tomaten, Gurken und Bohnen im Gefäßversuch. Landwirtsch. Forsch., Sonderh.37, Kongressband Braunschweig, 254-266

FOROUGHI, M., VENTER, F. und TEICHER, K., 1982: Experimentelle Ermittlung der Schwermetallanreicherung und -verteilung in Buschbohnen (Phaseolus vulgaris L.). Landwirtsch.Forsch., Sonderh. 38, Kongressband Trier, 239-248.

FRANZ-GEARG, L. und WERNER, H., 1995: Einfluß der VA-Mykorrhiza auf die Schwermetallaufnahme von Hafer (Avena sativa L.) in Abhängigkeit vom Kontaminationsgrad der Böden. Z.Pflanzenern. Bodenk. 158, 339-345.

GIORDANO, E., 1979: Aspetti selvicolturali del cipresso in Italia. In: „, II Cipresso: matattie e diffesa." Ed. GRASSO e RADDI, 79-86.

GOERGEN, G., 1987: Einfluß von Aluminium, Blei und Cadmium auf die Effizienz der VAMykorrhiza bei Capsicum annum und verschiedenen Boden-pH-Werten. Diplomarbeit, (Institut für Pflanzenbau und Tierhygiene in den Tropen und Subtropen) Göttingen.

GREJTOVSKÝ, A. and PIRC, R., 2000: Effect of high cadmium concentrations in soil on growth, uptake of nutrients and some heavy metals of Chamomilla recutita (L.) Rauschert. J.appl.Bot. 74, 169-174.

HAAN, S.de, 1972: Ergebnisse aus Versuchen mit Müllkompost. Landw. Forsch. 27, 68-92. 
HAGEMEYER, J., HEPPEL, T. and BRECKLE, S.W., 1994: Effects of Cd and Zn on the development of annual xylem rings of young Norway spruce (Picea abies) plants. Trees 8, 223-227.

HALL, N. and TURNBULL, J.W., 1976: Acacia saligna ( Labill.) H. Wendl. formerly known as A. cyanophylla Lindl?. Australian Acacias No. 4. CSIRO Division of Forest Research, Canberra.

HERMS, U. und BRÜMMER, G., 1978: Löslichkeit von Schwermetallen in Siedlungsabfällen und Böden in Abhängigkeit von $\mathrm{pH}$-Wert, Redoxbedingungen und Stoffbestand- Mitt.Dtsch.Bodenkundl.Gesellsch. 27, 23-34.

HERMS, U. und BRÜMMER, G., 1980: Einfluß der Bodenreaktion auf Löslichkeit und tolerierbare Gesamtgehalte an Nickel, Kupfer, Zink, Cadmium und Blei in Böden und kompostierten Siedlungsabfällen. Landw. Forsch. 33, 408-423.

HODENBERG, A.von, 1974: Ermittlung von Toxizitäts-Grenzwerten für Kupfer, Zink und Blei in Getreide, Rotklee und Rüben sowie Aufklärung der Toxizitätsschäden an Feldpflanzen im Harzvorland. Dissertation, Kiel.

HORAK, O., 1976: Bestimmung von Blei und Cadmium in Getreide- und Grasproben aus verschiedenen Entfernungen von Autostraßen mit Hilfe der flammenlosen AtomabsorptionsSpektroskopie. Landw. Forsch. 29, 289-298.

HU, H. and SPARKS, D., 1990: Zinc deficiency inhibits reproductive development in 'Stuart' pecan. Hort.Sci. 25, 1392-1396.

HU, H. and SPARKS, D., 1991: Zinc deficiency inhibits chlorophyll synthesis and gas exchange in 'Stuart' pecan. Hort.Sci. 26, 267-268.

JARVIS, S.C., JONES, L.H.P.and HOPPER, M.J., 1976: Cadmium uptake from solution by plants and its transport from roots to shoots. Plant and Soil 44, 179-191.

JOHN, M.K., 1973: Cadmium uptake by eight food crops as influenced by various soil levels of cadmium. Environ.Pollution 4, 7-15. 
JONER, E.J. and LEYVAL, C., 2001: Time-course of heavy metal uptake in maize and clover as affected by root density and different mycorrhizal inoculation regimes. Biol.Fertil.Soils 33, 351-357.

KABATA-PENDIAS, A., PENDIAS, H., 1984: Trace Elements in Soils and Plants; page 67. CRC Press, Boca Raton, Florida.

KABATA-PENDIAS, A. und PENDIAS, H., 1992: Trace Elements in Soils and Plants, $2^{\text {nd }}$ Ed., CRC Press, Boca Raton, Florida.

KAMPE, W., 1980: Schwermetallkontamination bei Pflanzen. Landwirtsch.Forsch., Sonderh. 36, Kongressband Gießen, 322-335.

KICK, H., 1974: Problematik der anorganischen Schadstoffe bei der Kompostierung von Siedlungsabfällen. Giessener Berichte zum Umweltschutz 4, 65-72.

KITSON, S.E. und MELLON, M.G., 1944: Colorimetric determination of phosphorus as molybdivanado phosphoric acid. Industr. Engineering Chem. Analyt. Ed. 16: 379-383

Kann so nicht stimmen

KLOBUS, G. and BUCZEK, J., 1985: Chlorophyll content, cells and chloroplast number and cadmium distribution in Cd-treated cucumber plant. Acta Physiol.Plant 7, 139-147.

KLOKE, A. und SCHENKE, H.D., 1979: Der Einfluß von Cadmium im Boden auf den Ertrag verschiedener Pflanzenarten und deren Cadmiumgehalt. Z.Pflanzenern.Bodenk. 142, 131-136.

KLOKE, A., 1981: Aufnahme umweltrelevanter Elemente durch die Pflanze. Deutsche Gesellschaft Qualitätsforschung (Pflanzliche Nahrungsmittel) e. V., XVII. Vortragstagung Siedlungsabfall-Verwertung und Nahrungsqualität am 26. und 27. März in Speyer.

LAG, J., HOATUM, O. und BOLVIKEN, B., 1970: Occurrence of naturally lead poisoned soil at Kastad near Gjövik, Norway. Norg.Geal. Unders. 266, 141-159

LAGERWERFF, J.V., 1971: Uptake of cadmium, lead and zinc by radish from soil and air. Soil Sci. 111, 129- 133.

LARCHER, W., 1975: Physiological Plant Ecology. Springer, Berlin, pp. 252. 
LAURENZ, L., 1978: Wirkung von Kompost aus Müll und Müllklärschlamm auf Ertrag, physikalische Bodenmerkmale, Mineralstoffversorgung und Schwermetallbelastung in Versuchen mit Getreide, Raps und Gras. Dissertation, Kiel.

LORENZ, S.E., HAMON, R.E., HOLM, P.E. DOMINGUES, H.C., SEQUEIRA, E.M., CHRISTENSEN, T.H. and McGRATH, S.P., 1997: Cadmium and zinc in plants and soil solutions from contaminated soils. Plant and Soil 189, 21-31.

LUCANBOUCHE, M.L., HABETS, F., BIAGIANTIRISBOURG, S. and VERNET, G., 1997: The simultaneous influence of $\mathrm{pH}$ and temperature on binding and mobilization of metals in sand. 2. Lead. Fresenius environ. Bull. 6(11-12), 711-718.

LUIS, E.H., ESTHER, L.-R., AGUSTIN, G. and RAMON, C.-R., 1998: Influence of cadmium on the uptake, tissue accumulation and subcellular distribution of manganese in pea seedlings. Plant Sci. 132, 139-151.

MALONE, C.P., MILLER, R.J. and KOEPPE, D.E., 1978: Root growth in corn and soybeans: effects of $\mathrm{Cd}$ and $\mathrm{Pb}$ on lateral root initiation. Can.J.Bot. 56, 277-281.

MANDAL, B. and HAZRA, G.C., 1997: Zinc adsorption in soils as influenced by different soil management practices. Soil Sci. 162, 713-721.

MASLIN, B.R., 1974: Studies in the genus Acacia. 3. The taxonomy of A. saligna (Labill.) H.L.Wendl. Nuytsia 1, 332-340.

MASSEY, H.F., 1972: pH and soluble $\mathrm{Cu}, \mathrm{Ni}$, and $\mathrm{Zn}$ in Eastern Kentucky Coal mine spoil materials. Soil Sci. 114, 217-221.

MAZEN, A.M.A. and EL MAGHRABY, O.M.O., 1997/98: Accumulation of cadmium, lead and strontium, and a(the?) role of calcium oxalate in water hyacinth tolerance. Biol.Plant. 40, 411-417. 
McGRATH, Z.G., SHEN and ZHAO, F.J., 1997: Heavy metals uptake and chemical changes in the rhizosphere of Thlaspi caerulescens and Thlaspi ochroleucum grown in contaminated soils. Plant and Soil 188, 153-159.

MENGEL, K. und STEFFENS, D., 1982: Beziehung zwischen Kationen /Anionen Aufnahme von Rotklee und Protonenabscheidung der Wurzeln. Z.Pflanzenern.Bodenk.145, 229-236.

MICHAELIDES, E.D., 1979: Mini-monograph on Acacia cyanophylla Technical Consultation on Fast-Growing Plantation Broadleaved Trees for Mediterranean and Temperate Zones. Lissabon, Portugal, 16-20 October 1979. FAO, Rome

MORAL, I., GOMEZ, J., NAVARRO, P. and MATAIX, J., 1994: Effects of cadmium on nutrient distribution, yield and growth of tomato grown in soilless culture. J.Plant Nutr. 17, 953-962.

N. A. S., 1980a: Firewood crops. Shrub and tree species for energy production. National Academy of Sciences, Washington, DC.

N. A. S., 1983b: Casuarinas: nitrogen fixing trees for adverse sites. National Academy Press, Washington, DC.

NEUMANN, U., 1980: Schwermetallgehalte in straßennahen Böden der Stadt Kiel. Diplomarbeit, Institut für Pflanzennährung und Bodenkunde der Universität Kiel.

POROKNEVICH, N.N., 1975: Structural organization and pigments of the plastid apparatus of a unit of the leaf surface and productivity of flat under different condition of zinc and copper nutrition. S-KH Biol., 10 (4) : 511-517.

PURIC, O., 1967: Cupressus sempervirens in Belgrade. Sumarstavo 20, 27-32.

RADDI, P. and PANCONESI, A., 1990: Genetic tolerance to cold in Cupressus sempervirens progenies. Silvae Gen. 38, 168-172. 
RIEDER, W. und SCHWERTMANN, U., 1973: Kupferanreicherung in hopfengenutzten Böden der Hallertau. Landw. Forsch. 25, 170-177.

ROSOPULO, A. und DIEZ, TH., 1982: Die Anreicherung von Schwermetallen verschiedener auf kontaminierten Böden angebauter Pflanzen. Landw. Forsch., Sonderh. 38, Kongressband Trier, 751-767.

ROSS, S.M. and KAYE, K.J., 1994: The meaning of metal toxicity in soil-plant systems. In: ROSS, S.M., (Ed.). Toxic Metals in Soil-Plant systems; S.27-61. Wiley, Chichester, England.

SCHOEMAKERS, J., 1986: Einflußfaktoren für die Aufnahme von Schwermetallen durch Pflanzen. Kali-Briefe (Büntehof) 18(5), 383-395.

SHABAN, SH.A. and EID, A., 1982: Effect of some micronutrients on seed germination and seedling growth in maize and cotton. Res.Bull., Ain Shams Univ., Fac. of Agric., Egypt.

SIMMONS, M., 1981: Acacia of Australia. Nelson, Melbourne.

SINGER, M.J. und HANSON, L., 1969: Lead accumulation in soils near highways in the Twin cities metropolitan area. Soil Sci.Soc.Am.Proc. 33, 152- 153.

SOMMER, G., 1979: Gefäßversuche zur Ermittlung der Schadgrenzen von Cadmium, Kupfer, Blei, und Zink im Hinblick auf den Einsatz von Abfallstoffen in der Landwirtschaft. 2. Mitt.Landwirtsch.Forsch., Sonderh. 35, Kongressband Augsburg, 350-364.

STENSTRÖM, T. und VAHTER, M., 1974: Cadmium and lead in Swedish commercial fertilizers. Ambio 3, 391-392.

STICHER, H., 1980: Schadstoffe im Boden: Wann können sie in die Nahrungskette gelangen? Schweiz.Landw.Forsch. 19. Sonderh. 125 Jahre ETH, 267-279.

TAHA, R.A., 1994: Seed Germination and Seedling Growth of some Ornamental Trees. M.Sc. Thesis, Fac. of Agric., Minia Univ. Egypt

TAKIJIMA, Y. und KATSUMI, F., 1973: Cadmium contamination of soils and rice plants caused by zinc mining. I. Production of high cadmium rice on the paddy fields in lower reaches(?) of the mine station. Soil Sci.Plant Nutr. 19, 29-38. 
TICHÝ, R., FAJTL, J., KUZEL, S. and KOLAR, L., 1997: Use of elemental sulphur to enhance a cadmium solubilization and its vegetative removal from contaminated soil. Nutrient Cycling Agroecosystems 46, 249-255.

TURNBULL, J.W. (ed), 1987: Australian Acacias in Developing Countries. ACIAR Proceedings No. 16. ACIAR, Canberra, Australia.

VAN BALEN, E., VAN DE GEYN, S.C. and DESMET, G.M., 1980: Autoradiographic evidence for the incorporation of cadmium into calcium oxalate crystals. Z. Pflanzenphysiol. $97,127-133$

VAN LUNE, P. and ZWART, K.B., 1997: Cadmium uptake by crops from the subsoil. Plant and Soil 189, 231-237.

VAN STEVENINCK, R.F.M. and FERNANDO, D.R., 1995: X-ray microanalyticial studies on two modes of strontium binding in fronds of Lema minor. Aust. J. Plant Physiol. 22, 817822

VENTER, F., 1980: Zusammenhang zwischen Standort und Schwermetallgehalt in Gemüse. Landwirtsch.Forsch., Sonderh. 36, Kongressband Gießen, 336-342.

VERKLEIJ, J.A.C. and SCHAT, H., 1990: Mechanisms of metal tolerance in higher plants. In: SHAW, A.J. (Ed.): Heavy Metal Tolerance in Plants: Evolutionary Aspects; S., 179-193. CRC Press, Boca Raton, Florida.

VETTER, H. und MÄHLHOP, R., 1971: Untersuchungen über Blei-, Zink und Fluorimmissionen und dadurch verursachte Schäden an Pflanzen und Tieren. Landwirtsch. Forsch. 24, 294-315.

WEISCHET, W., 1984: Schwiergkeiten tropischer Bodenkultur. Spektrum der Wissenschaft $7, \mathrm{~S}, 112-122$

YANG, X., BALIGAR, V.C., MARTENS, D.C. and CLARK, R.B., 1996: Cadmium effects on influx and transport of mineral nutrients in plant species. J.Plant Nutr. 19, 643-656.

YOUNG, J.P.W., 1996: Phylogeny and taxonomy of rhizobia. Plant and Soil 186, 45-52. 


\section{Lebenslauf}

Name : $\quad$ Ragaa Aly Taha Aly

Geboren : $\quad 17.10 .1967$ in El-Minia/Ägypten

Familienstand : $\quad$ verheiratet, vier Kinder

Schulen : $\quad 1974-1980$ Grundschule in El-Minia

1980 - 1983 Mittelschule in El-Minia

1983 - 1985 Gymnasium in El-Minia

01 .08. 1985 Abitur in El-Minia

Studium :

1985-1989 Studium an der Landwirtschaftliche Fakultät der Universität von El-Minia ( B.Sc. degree )

1990 -1994 M.Sc.- Studium an der Landwirtschaftliche Fakultät der Universität von El-Minia

1990-1997 Dozentin an der Landwirtschaftliche Fakultät der Universität von El-Minia

Promotion : $\quad$ Seit 1997 Doktorandin im Bereich Pflanzenbau, Grisebachstr. 6, des Instituts für Pflanzenbau und Tierproduktion in den Tropen und Subtropen der Universität Göttingen 
Danksagungen

Herrn Prof. Dr. P.L.G. Vlek gilt mein herzlicher Dank für das entgegengebrachte Vertrauen und die Unterstützung bei der Durchführung dieser Arbeit.

Ferner danke ich Herrn Prof. Dr. W. Römer für die freundliche Übernahme des Korreferates.

Mein besonderer Dank gilt Herrn Dr. A.M. Moawad für die kritische Durchsicht des Manuskripts und die jederzeit gewährte Hilfsbereitschaft.

Ein besonderer Dank gilt Herrn Dr. M. Denich für seine Hilfe.

Ich möchte mich bei Herrn Dr. H. Koch für seine Hilfe bei der Durchsicht des Manuskripts bedanken.

Allen Institutsangehörigen danke ich für ihre Hilfsbereitschaft, besonders Frau Dr. Schultz.

Viel mehr als sich mit Worten sagen läßt, gilt letztlich mein Dank meinem Mann und meinen Kindern. 